


\section{Mild Oxidation and Functionalisation of Synthetic Polymer Surfaces}




\section{Thesis committee}

\section{Promotor}

Prof. Dr J.T. Zuilhof

Professor of Organic Chemistry

Wageningen University \& Research

\section{Co-promotor}

Dr M.C.R. Franssen

Associate professor at the Laboratory of Organic Chemistry

Wageningen University \& Research

\section{Other members}

Prof. Dr W.J .H. van Berkel, Wageningen University \& Research

Prof. Dr H.D.W. Roesink, University of Twente

Prof. Dr G.M. Gübitz, University of Natural Resources and Life Sciences, Vienna, Austria

Dr F. Hollmann, Delft University of Technology

This research was conducted under the auspices of Graduate School VLAG (Advanced Studies in Food Technology, Agrobiotechnology, Nutrition and Health Sciences). 


\title{
Mild Oxidation and Functionalisation of Synthetic Polymer Surfaces
}

\author{
Sjoerd Slagman
}

Thesis

submitted in fulfilment of the requirements for the degree of doctor at Wageningen University

by the authority of the Rector Magnificus,

Prof. Dr A.P.J. Mol,

in the presence of the

Thesis Committee appointed by the Academic Board

to be defended in public

on Wednesday 7 November 2018

at 4 p.m. in the Aula. 


\section{Sjoerd Slagman}

Mild Oxidation and Functionalisation of Synthetic Polymer Surfaces

164 Pages.

PhD thesis, Wageningen University, Wageningen, the Netherlands (2018)

With references, with summary in English

ISBN 978-94-6343-511-6

DOI https://doi.org/10.18174/459469 




\section{Table of contents}

Chapter 1

Chapter 2

Chapter 3

Chapter 4

Chapter 5

Chapter 6

Summary

Acknowledgements

Publications

About the author

Overview of completed training activities

General discussion oligomerisation of 4-hydroxybenzoic acid

Elucidating the mechanism behind the laccasemediated modification of poly(ethersulfone)

Supporting information

67

Laccase-mediated grafting on biopolymers and synthetic polymers: a critical review

One-step generation of reactive superhydrophobic surfaces via $\mathrm{SiHCl}_{3}$-based silicone nanofilaments

Supporting information

Supporting information 
Chapter 1

General introduction 


\section{Surface chemistry}

When imagining what a chemist does, most people will envision someone in a white lab coat working with strange glassware containing strongly coloured liquids. ${ }^{[1]}$ Although chemistry in the $21^{\text {st }}$ century cannot be summarised in one such picture, it does, in its essence, quite accurately cover one of its most important sub-domains: synthetic chemistry. A synthetic chemist indeed makes new molecules by reacting together two (or more) others. By doing so, he or she is able to make, for example, drugs, dyes, polymers or explosives. What is also sketched in the general example of a chemist, is solution-based synthesis. Solution-phase chemistry concerns reactions in which all molecules of the starting compound have approximately the same properties and are allowed to move, orient, wiggle, stretch and fold freely in solution. This thus means that every molecule is equally likely to react in the same way, as all molecules have the opportunity to position themselves optimally before reacting (Scheme 1 ).

Another sub-domain of chemistry, that is increasingly gaining importance, is the field of surface chemistry. Surface chemistry is the study of chemical phenomena at the interface (i.e. solid/liquid). This encompasses, amongst others, the analysis of chemical surface properties of paintings, ${ }^{[2]}$ the use of surface-bound species to perform catalysis ${ }^{[3]}$ and the modification of molecules on the surface. Altering molecules at the interface often concerns the modification of molecules on a solid surface, which is of utmost importance for the modification of a wide variety of materials, such as silicon, ${ }^{[4]}$ glass, ${ }^{[5]}$ polysaccharides ${ }^{[6]}$ and graphene. ${ }^{[7]}$ Enhancing durability, ${ }^{[8]}$ inducing anti-microbial activity, ${ }^{[9]}$ altering catalytic activity ${ }^{[10]}$ or changing wettability ${ }^{[1]]}$ of a material could all be achieved through a change in surface chemistry.

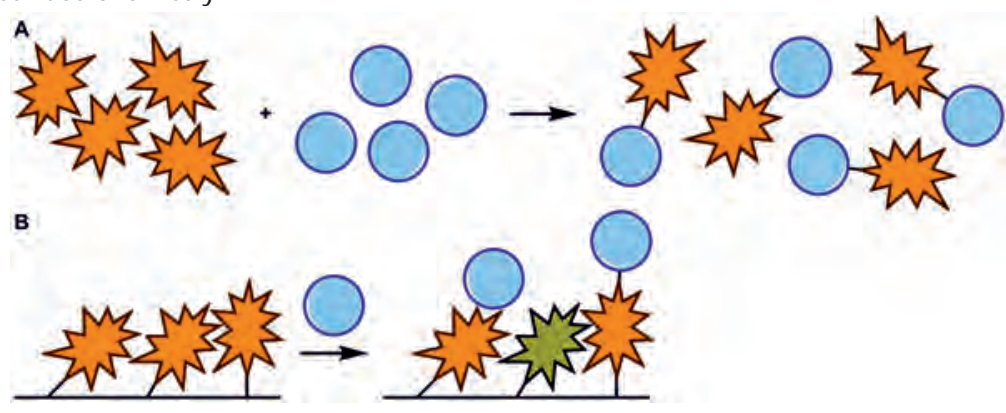

Scheme 1. A: Idealised solution-phase synthesis in which all starting material reacts to product. B: A surface chemical reaction in which the orientation of certain molecules restricts them from being converted to the desired product.

Chemically modifying the surface of a material can be achieved in various ways. For ages, humankind has used dyes to colour the surface of textiles, paintings or walls. However, dyeing is often a mere adsorptive process and thus the result of many, but individually weak interactions. ${ }^{[12]}$ Consequently, in order to obtain more durable coatings, one would preferably modify the surface covalently. Covalent bonds are bonds between atoms in one molecule in which these atoms share an electron pair. ${ }^{[13]}$ Covalent bonds are far stronger than the weak interactions e.g. present between an adhesive like super-glue and the materials it glues together. Many of the reactions performed by the synthetic chemist, in order to make small-molecule drugs, for example, involve the making of a covalent bond. The surface chemist, however, aims to modify extremely big molecules (i.e. silicon crystals, plastics or wood), which do not behave like independent entities in solution, ${ }^{[14]}$ as was the case for the molecules described before. In such a solid material every sub-unit (or in the case of polymers: monomer), has different properties than its neighbour, even though its chemical structure might be exactly the same.[15] Its mobility is restricted and its chemical accessibility is different; therefore, each sub-unit might react differently. These surface-bound molecules might, for example, undergo intramolecular alterations, merely adsorb molecules from the reaction mixture or prove to be completely unreactive due to steric hindrance from adjacent sub-units (Scheme 1). 
Additionally, the surface chemist aims to only chemically modify the exterior of a material; internal alterations might even have adverse effects, such as inducing brittleness or decreasing material strength. ${ }^{[16]}$ These issues are of reduced importance when considering modifications on dense solid materials like silicon or mica, but are more pronounced when altering porous polymers such as gels or membranes. Altogether, (covalently) modified surfaces prove to be all around us, but their development does not come about easily.

\section{Polymer surface wettability and analysis}

Polymers play a unique role in material science, as they are chemically and structurally very diverse due to the availability of many different monomers. Their diversity allows polymers to be broadly applicable. Well-known examples are nylon textiles, poly(ethylene terephthalate) recyclable bottles, and Teflon ${ }^{\mathrm{TM}}$ (poly(tetrafluoroethylene), PTFE) anti-stick coatings (Scheme 2). Unwanted adhesion to polymers, or to any other material for that matter, is a major issue regarding their applicability. Where Teflon ${ }^{T M}$ is a polymer that on itself acts as a coating to prevent unwanted adhesion, other polymers have to be modified in order to minimise adhesion. One such a polymer for which this is often required is poly(methyl methacrylate) (PMMA), a polymer that is frequently used outdoors as it is cheap, strong and highly transparent. ${ }^{[17]}$ Much effort is put forth in preventing water adhesion to PMMA in order to maintain its transparency and/or to add self-cleaning properties to this material so it can withstand (extreme) weathering. ${ }^{[11,18]}$ Similarly, water filtration membranes are often made of inert polymers like poly(ethersulfone) (Scheme 2), which are highly stable but suffer severely from fouling. ${ }^{[19]}$ Fouling is the adsorption of unwanted material on a surface. Membranes most commonly suffer from unwanted adhesion of biofoulants such as polysaccharides, polyphenols, proteins and microbes.

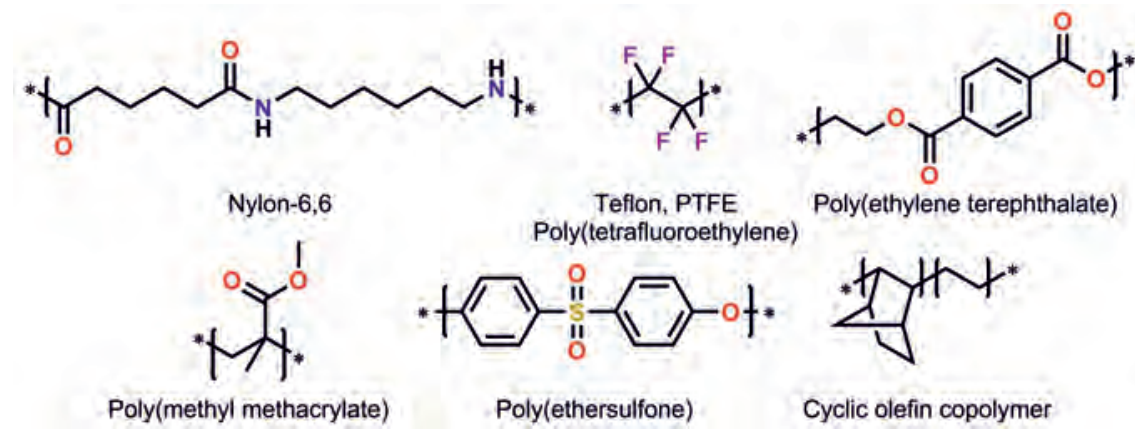

Scheme 2. Selected examples of commonly used polymers.

Fouling prevention correlates strongly with control over surface wettability. ${ }^{[20]}$ Wettability is eminently a surface property and thus often tuned through chemical surface alterations. The polymer Teflon $^{\mathrm{TM}}$, for example, is highly hydrophobic and a water droplet deposited on its surface therefore minimises contact with the surface. Teflon ${ }^{T M}$ exhibits a so-called static water contact angle (SWCA) of $120^{\circ}{ }^{[21]}$ The SWCA is the angle measured between the surface and the droplet's contour (Figure 1 ). The SWCA is characteristic for the surface under the employed measurement conditions and therefore a rapid surface analysis tool. ${ }^{[22]}$ Surfaces exhibiting SWCA's $<90^{\circ}$ are deemed hydrophilic, and when these angles are too low to measure accurately $\left(\sim 15^{\circ}\right)$, these surfaces are called superhydrophilic. Conversely, SWCA's of $90-150^{\circ}$ correspond to hydrophobic surfaces, while superhydrophobic surfaces exhibit SWCA's $>150^{\circ}$.

Wettability is, furthermore, influenced by surface topography. Introducing nano- or microscale roughness could make the surface superhydrophobic.[23] In other words, a perfectly smooth surface will never exhibit SWCA's of approximately $123^{\circ}$ or higher, ${ }^{[11]}$ while nanostructured surfaces might. When surface texture is such that water is not able to penetrate within, it additionally induces self- 
cleaning properties. ${ }^{[24]}$ Due to this so-called lotus effect, droplets slide off, even when applying only minimal force (through tilting for example).

A

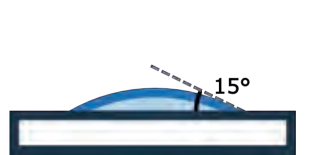

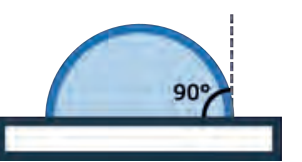

C

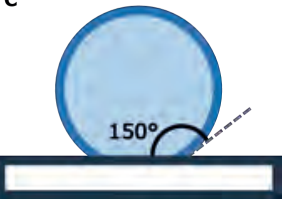

Figure 1. Schematic depiction of a water droplet on surfaces with distinct wetting properties. Superhydrophilic surfaces have SWCA's of less than $\sim 15^{\circ}$ (A), hydrophilic surfaces have SWCA's between $15^{\circ}(\mathrm{A})$ and $90^{\circ}$ (B), hydrophobic surfaces have SWCA's between $90^{\circ}$ (B) and $150^{\circ}$ (C) and superhydrophobic surfaces have SWCA's of more than $150^{\circ}$ (C).

Conversely, increasing surface hydrophilicity can also prevent unwanted adhesion. ${ }^{[20,25]}$ Initial biofouling of materials occurs when the hydrophobic portion of a protein interacts strongly with a hydrophobic surface such as a hydrocarbon polymer. ${ }^{[26]}$ This is similar to aggregation of oil droplets in water, and is called the hydrophobic effect. By making the surface more hydrophilic, the material's exterior will be solvated more extensively through the formation of a hydration layer in aqueous media, which minimises initial protein adsorption. Common hydrophilisation agents are immobilised poly(ethylene glycol) or zwitterionic brushes. ${ }^{[27]}$ Once again, SWCA measurements are useful for characterising the induced chemical change as one modifies a hydrophobic surface such that it becomes hydrophilic.

Apart from analysing the effect of chemical alterations on physical material properties, as is the case for SWCA measurements, one preferably also analyses the chemical change directly. Unfortunately, current state of the art in molecular analysis does not allow for facile analysis on individual (complex) molecules. Therefore, analysing molecular change usually occurs on a multitude of molecules at once. As mentioned, small-molecules in solution are highly mobile; when analysing them, they therefore on average all look the same, which commonly eases analysis. This could be compared to taking a picture at very slow shutter speeds. Analysing the molecular nature of chemical alterations on a surface is, however, often more challenging than analysing that of entities in solution; ${ }^{[22]}$ molecules on a solid surface have restricted mobility, a snapshot of each of these molecules looks different. As analysis of all these molecules occurs at once, one thus has to overlap these snapshots, resulting in a very blurry image. Therefore, a technique such as nuclear magnetic resonance (NMR) spectroscopy, which is extremely powerful for characterising molecules in solution, is not optimal for determining the chemical structure of molecules on a surface.

A tool that is being used routinely to analyse chemical alterations on a surface is X-ray photoelectron spectroscopy (XPS). ${ }^{[22]}$ XPS analysis is used to probe the elemental composition of the exterior of an analyte. An X-ray source fires high-energy $X$-ray beams at the sample surface and thereby emits core shell electrons (those tightly bound to the nucleus) from their respective atoms (Figure 2). Electrons from distinct orbitals possess a distinct amount of kinetic energy after being emitted, which is measured by a detector. As the kinetic energy is directly proportional to the binding energy, one is thus able to determine the element of which the electron is emitted. When measuring at higher resolution, one is, furthermore, able to determine the atom's chemical surroundings (what other atoms it binds to; nature of functional group which it is part of). As some elements give rise to more intense signals, these elements can be introduced on the surface as tags. Fluorine is a particularly popular tag because its detection does not result in multiple signals as commonly only detection of electrons from its $1 \mathrm{~s}$ shell results in an intense signal (where heavier elements give multiple signals). Upon X-ray impact, electrons emitted from the analyte travel through the material to eventually escape into a strong vacuum before being detected. The longer the path the electron has to travel (i.e., when it originates from deeper in the material), the lower the chance that it reaches the detector as it loses its energy by colliding with other particles for example. This makes XPS a surfacesensitive technique, as electrons escaping from approximately $10 \mathrm{~nm}$ deep or deeper have a much 
lower change of being detected, and - in case they are - do not contain much element-specific information anymore.

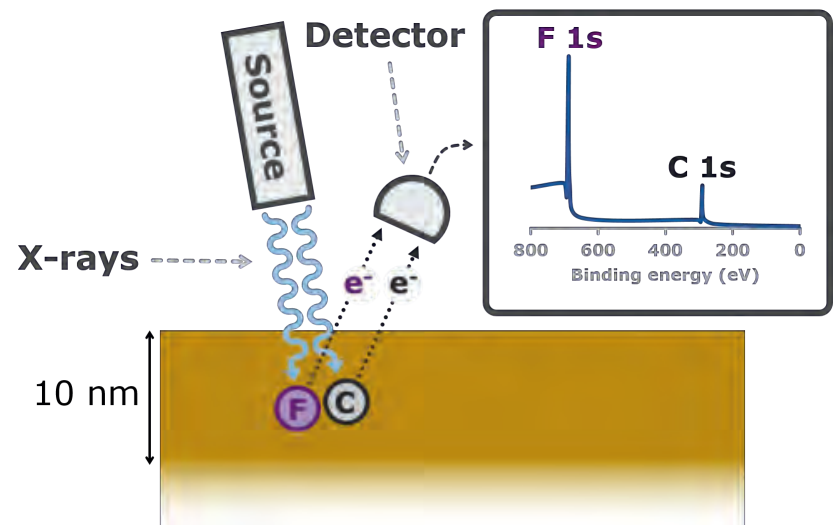

Figure 2. Schematic depiction of XPS wide scan (low resolution) analysis on an imaginary solid surface built up solely from equimolar amounts of carbon and fluorine. The XPS signals are annotated with the chemical symbol and electron shell of the atom from which the respective electrons have been emitted. Please note that the fluorine signal is approximately 4.5 times more intense.

\section{C-H bond activation on synthetic polymers}

As the chemical nature of polymers is highly diverse, one is able to develop materials that have many valuable properties, like high strength or anti-bacterial activity, from scratch. However, polymers that meet these high demands are built up from complex monomers that are difficult and costly to obtain. Furthermore, as only some of this material is on the exterior, much of it is very expensive bulk. Polymer surface chemistry could circumvent these issues, as it only alters the polymer exterior and often does not require polymers made from custom-designed monomers. ${ }^{[28]}$

Conversely, many applications require chemically inert polymeric materials to ensure durability. As these inert polymeric materials often do not bear reactive handles (functional groups), post-production covalent modifications are hampered by this inertness. Exemplary inert polymers are polypropylene, poly(ethersulfone) and cyclic olefin copolymer (see Scheme 2), which mainly consist of unreactive C-C and $\mathrm{C}-\mathrm{H}$ bonds. $\mathrm{C}-\mathrm{H}$ bond activation is therefore required to allow for subsequent modifications of the polymer. C-H bond activation (or activation for short) is the cleavage of a $\mathrm{C}-\mathrm{H}$ bond and subsequent formation of a C-Y bond in which $\mathrm{Y}$ could be any element, but commonly oxygen, nitrogen or carbon. ${ }^{[29]} \mathrm{As}$ the $\mathrm{C}-\mathrm{H}$ bond is a strong bond (hence its inertness) it requires a lot of energy to break.

A popular and easily applicable method to modify surface properties and allow for further surface chemistry to be conducted, is applying an oxygen or air plasma. ${ }^{[30]}$ The (polymer) material that is to be activated is placed in a vacuum chamber, in which either of the mentioned gases is subsequently excited through radiation, resulting in the formation of, among others, oxygen-centred radical species (Scheme 3). These radicals subsequently react with the polymer surface to break its $\mathrm{C}-\mathrm{H}$ bonds, and form a plurality of oxygen-rich functional groups, such as hydroxyls, peroxides and aldehydes on the surface. ${ }^{[31]}$ Alternatively, one could introduce amines on the surface by employing ammonia or nitrogen plasma. ${ }^{[32]}$ Additionally, grafting (covalently binding polymers to a surface) can be achieved directly by creating surface-bound radicals using (argon) plasma before supplying a monomer of which polymerisation is initiated from the surface (Scheme 3). ${ }^{[33]}$

Besides oxygen or air plasma, there are many other methods to introduce oxygen-rich functional groups on a polymer surface. These methods commonly rely on thermal oxidation using strong oxidising agents such as persulfates or ozone. ${ }^{[34]}$ As with plasma treatment, harsh chemical agents might introduce extensive bond-cleavage and induce topographical irregularities, indicating unwanted adsorption. ${ }^{[35]}$ 
A

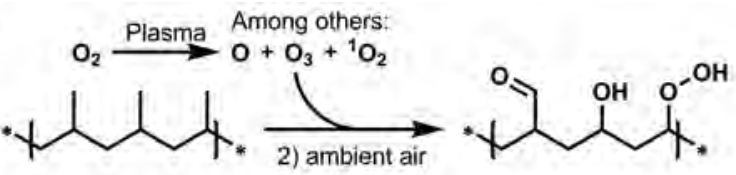

c<smiles>[O-]c1ccccc1</smiles><smiles>[O-]c1ccccc1O</smiles>

B

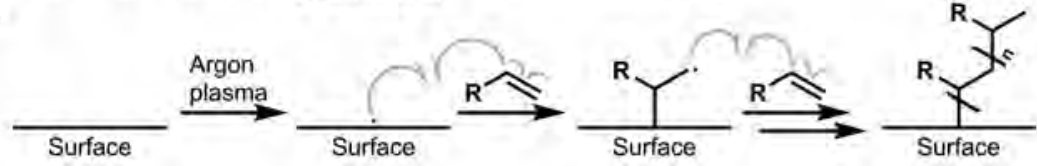

Scheme 3. A: Plasma generation of reactive oxygen species that subsequently react with a polypropylene surface to form oxygen-rich functional groups, such as aldehydes, hydroxyls and peroxides.*[31] B: General polymerisation mechanism depicting initiation and propagation from plasma-induced surface radicals, which is eventually followed by termination to result in a surface-grafted polymer. C: Oxygen-centred phenoxyl radical of phenol and catechol. *The depicted structure is a mere example of possible functionalisation as interpreted by us.

Recently, mild surface oxidation under Fenton-like conditions using a commercially available copper catalyst has proven to be among the mildest of methods of activating an all-carbon polymer (cyclic olefin copolymer) (Scheme 4). ${ }^{[36]}$ Subsequently, performing a mild reduction using $\mathrm{NaBH}_{4}$, exclusively yields hydroxy groups on the surface. The latter trait, in combination with being suitable for the activation of the inside of intricate microfluidic channels, makes this oxidation/reduction strategy highly interesting for activating inert polymers.

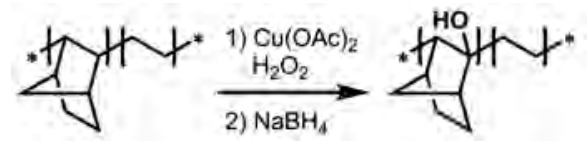

Scheme 4. Oxidation/reduction strategy for generating hydroxy groups on the cyclic olefin copolymer surface. ${ }^{[36]}$ The depicted hydroxylation site is based on speculations by us.

UV irradiation of a polymeric surface is another way of achieving C-H bond activation. ${ }^{[37]}$ In some cases in the presence of a photoinitiator, UV irradiation generates surface-bound radicals that can subsequently couple to monomers or other molecules in solution. Other methods of radical-induced C$\mathrm{H}$ activation include the (thermal) homolytic bond cleavage of aryl diazonium salts to form $\mathrm{N}_{2}$ and the respective phenyl radical, which can act as radical initiator or be coupled to the surface directly. ${ }^{[38]}$ Unfortunately, most of the methods mentioned above require harsh chemical agents that are not compatible with sensitive polymers such as PMMA, ${ }^{[17]}$ or make use of high-energy irradiation or plasma, resulting in chain scission and/or yellowing of the polymer. ${ }^{[39]}$ Milder methods to generate radicals in order to modify the exterior of polymers are thus highly desirable. Enzymes might offer a mild and eco-friendly alternative as they commonly operate in aqueous media and under ambient conditions. The enzyme laccase, which is able to generate phenoxyl radicals from phenol (hydroxybenzene) and catechol (1,2-dihydroxybenzene) (Scheme 3), has already been used to modify cellulose and lignin (polyphenolic support tissue of, among others, wood and grasses), ${ }^{[40]}$ and is currently employed in attempts towards the functionalisation of synthetic polymers. ${ }^{[41]}$

The term 'activation' is being used differently within distinct chemical disciplines. Throughout this thesis, (surface) activation encompasses those reactions in which a $\mathrm{C}-\mathrm{H}$ bond undergoes a functional group transformation or $\mathrm{H}$-abstraction in order to introduce a chemical anchor to allow for subsequent chemistries on the surface. Terms such as 'modification', 'functionalisation' and 'grafting' are used to describe those chemistries. 


\section{The enzyme laccase}

Many metabolic processes in nature are catalysed by enzymes. These enzymes are, outside of their native context, increasingly being used to also catalyse non-natural reactions on demand. Modern-day biochemistry allows for their isolation from their host organisms and even the design of new enzymes from scratch. Enzymes are now routinely used as medicinal agents, ${ }^{[42]}$ as part of laundry detergents ${ }^{[43]}$ or to minimise staling of bread. ${ }^{[44]}$ Their large applicability in combination with increased reusability due to immobilisation also allows for their use as laboratory or industrial biocatalyst to perform synthetic transformations. ${ }^{[45]}$

The family of laccases (EC 1.10.3.2) is one of these widely applicable enzyme families. ${ }^{[41 b, 46]}$ Laccases fall within the class of oxidoreductases: enzymes that catalyse the transfer of electrons from reductant to oxidant. ${ }^{[47]}$ More specifically, laccases are oxidases, as they use molecular oxygen as oxidant. They are found in a wide range of species, ${ }^{[48]}$ but those most commonly used are isolated from fungi. ${ }^{[49]}$ Laccases are of particular interest, as they commonly operate at nearly neutral $\mathrm{pH}$ and at room temperature. ${ }^{[47]}$ Furthermore, they allow reactions to be performed in aqueous media, but are also rather tolerant towards organic co-solvents. ${ }^{[50]}$ They are ideal catalysts for performing green chemistry as they only form water as by-product. ${ }^{[47]}$ They are, furthermore, able to convert a wide range of phenolics, the scope of which is widened even further when using laccase-mediator systems in which laccase does not oxidise the target directly, but oxidises a small-molecule that thereafter performs a one- or two-electron oxidation of the target.

The majority of fungal laccases are blue multicopper oxidases, which generally bear four copper atoms in their active sites. ${ }^{[51]}$ The copper ion residing at the so-called T1 site (scheme 5) is responsible for the intense blue colouration of the pure enzyme. Oxidation of the phenolic target molecule occurs at this site. Its redox potential can differ tremendously among different laccases and can be as high as $790 \mathrm{mV}$ (vs NHE; laccase from Trametes versicolor) ${ }^{[52]}$ and as low as $410 \mathrm{mV}$ (vs NHE; laccase from Rhus vernicifera). ${ }^{[53]}$ The other three copper atoms form a trinuclear cluster confined by multiple histidine residues, a motive that is highly conserved across the family of laccases. ${ }^{[49 b]}$ Two of these copper sites are so-called T3 sites, and the other is a T2 site at which molecular oxygen is reduced to water.

Dependent on their redox potential, laccases are able to convert merely catechols, or also phenols and even various less reactive moieties. ${ }^{[47]}$ Oxidation of a phenol at the $\mathrm{T} 1$ site results in its respective radical cation, which thereafter rapidly loses a proton (Scheme 5). The formed radical is then able to dimerise with another phenol in solution, be attacked by water (hydroxylation) or, when a susceptible (polymeric) material is present, form a covalent bond with molecules at the exterior of this material for example (Scheme 6). ${ }^{[54]}$ As the formed radical is delocalised over the phenolic system, dimerisation and subsequent oligomerisation often results in a highly heterogeneous product mixture. ${ }^{[55]}$

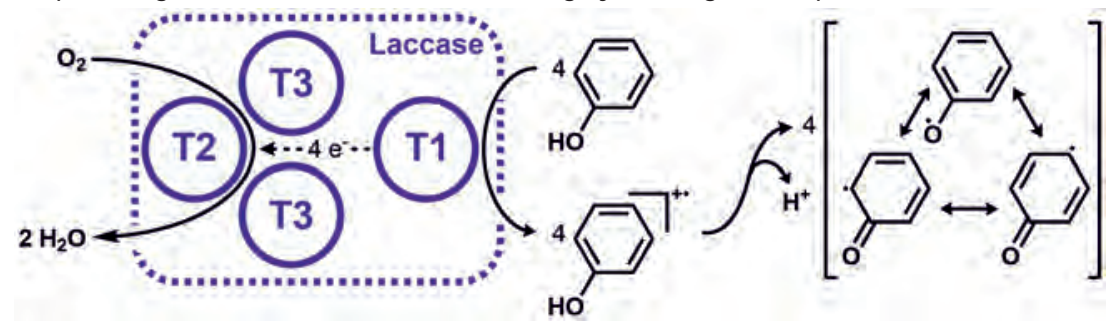

Scheme 5. General mechanism for the laccase-mediated conversion of four molecules of phenol to their respective radical cations that rapidly lose a proton to form delocalised phenoxyl radicals. Concomitant with the formation of the four radical cations is the reduction of oxygen to two molecules of water. 


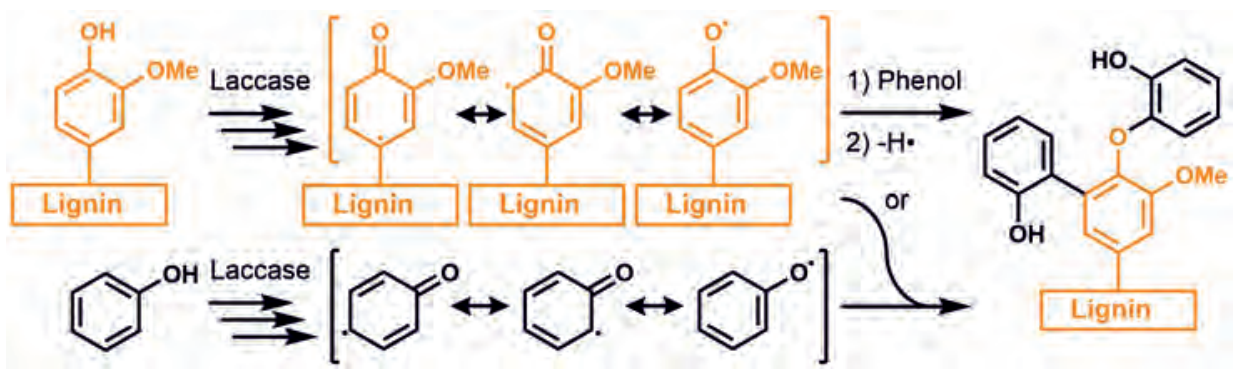

Scheme 6. Generic mechanism for the laccase-mediated conversion of phenol and a (chemically accessible) lignin unit resulting in coupling of the two (grafting). Product formation as a result of both plausible $\mathrm{C}-\mathrm{C}$ as well as $\mathrm{C}-\mathrm{O}$ bond formation is shown.

\section{Applications of laccase}

As laccases are able to convert a broad range of phenols and non-phenols, they are multipurpose catalysts that are employed in a wide variety of applications. By appreciating that their main mode of action in fungi is to depolymerise lignin to smaller nutrients, ${ }^{[49 a]}$ it is evident that laccases are often used in biomass processing. ${ }^{[56]}$ A laccase-generated (phenoxyl) radical is able to initiate bond formation, but also bond cleavage. ${ }^{[57]}$ Laccases are, for example, able to delignify lignocellulosic material to enhance sugar accessibility, and thereby facilitate their production. ${ }^{[58]}$ Conversely, lignocellulosic materials commonly also contain small toxic phenols; laccase is able to detoxify these materials by polymerising those phenols. ${ }^{[59]}$

Completely in line with its detoxification potential, bioremediation of soil or wastewater effluents is among the most common applications of laccase. ${ }^{[60]}$ In that regard, laccase has proven to efficiently remove oestrogens and other recalcitrant drugs from waste streams. ${ }^{[61]}$ Removal and detoxification of polyaromatic hydrocarbons ${ }^{[62]}$ and (textile) dyes using laccase has been proven to be an excellent bioremediation tool. ${ }^{[63]}$ Bleaching of pulp or denim to aid in paper and textile manufacturing through the laccase-mediated removal of (small-molecule) chromophores is among the many other applications of this highly diverse enzyme. ${ }^{[64]}$

It is, furthermore, compelling that laccases are not only used for breaking down dye molecules, but also for building them up. ${ }^{[65]}$ Extension of a $\pi$ system through C-C bond formation between multiple phenolic monomers leads to intense colouration. ${ }^{[66]}$ Laccases are also commonly used for the synthesis of active pharmaceutical intermediates or drug candidates. ${ }^{[67]}$ Laccase-mediated dimerisation or oligomerisation of antioxidants often further increases their antioxidant properties. ${ }^{[68]}$ Laccase has additionally been proven to be a more selective agent than bleach in the oxidation of (poly)saccharides. ${ }^{[69]}$

As touched upon earlier, laccase-mediated grafting allows for the mild functionalisation of lignin (Scheme 6). In this way properties such as wetting behaviour and anti-microbial activity can be

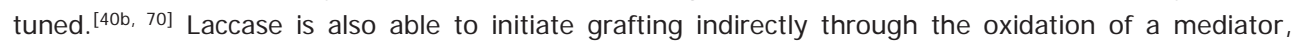
which then acts as a radical transfer agent or performs a two-electron oxidation on e.g. cellulose or lignin. ${ }^{[71]}$ The scope of laccase-mediated grafting has been broadened to include materials such as chitosan, ${ }^{[72]}$ wool[73] and silk. ${ }^{[74]}$ These materials are easily functionalised due to abundantly present nucleophilic amine groups.

More challenging is activation of, and subsequent grafting onto, inert synthetic polymers. Within our group we have employed the in-situ laccase-mediated oligomerisation of 4-hydroxybenzoic acid to functionalise inert poly(ethersulfone) membranes, which conferred anti-fouling properties to these membranes. ${ }^{[41 a, 75]}$ It was hypothesised that 4-hydroxybenzoic acid radicals could covalently bind to the membrane surface (Scheme 7). Direct analysis of the formed covalent bond is, however, challenging due to a limited availability of appropriate techniques to probe the nature of this bond and the resemblance between potentially grafted molecules and oligomeric molecules adsorbed on the 
surface via strong physisorption. To advance the field of laccase-mediated grafting, the underlying mechanisms should therefore be studied more extensively.

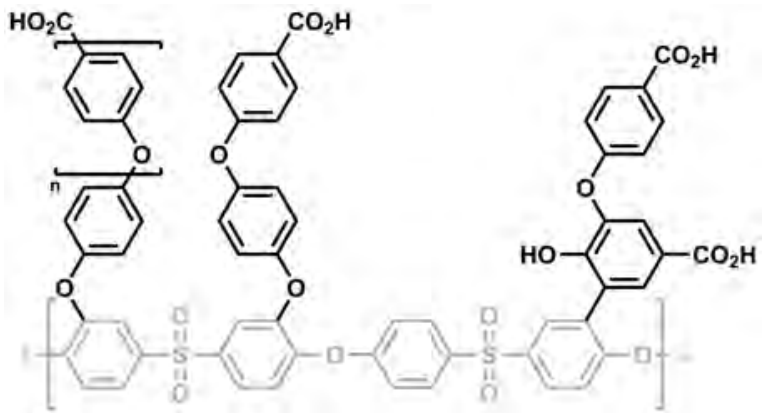

Scheme 7. Laccase-mediated grafting of 4-hydroxybenzoic acid on poly(ethersulfone) was proposed to result in brush-like oligomers on the surface. ${ }^{[41 a]}$

\section{Aim of this thesis}

Post-production modification of synthetic polymers relies heavily on the use of plasma activation or UV irradiation, as both easy-to-use techniques provide enough energy to break $\mathrm{C}-\mathrm{H}$ bonds to install chemical anchors and thereby allow for further functionalisation. However, these, and many other methods, rapidly induce chain scission, yellowing and/or roughening of the polymer; mild polymer activation strategies are therefore highly desirable. With the work described in this thesis, we aimed to get a better mechanistic understanding of mild enzymatic polymer functionalisation in order to further expand its scope. We alternatively intended to develop chemical strategies for polymer functionalisation in order to contribute to a "toolbox" filled with multiple tools that would allow for mild and tailor-made polymer modification. By employing these tools, we aim to control polymer wettability and adhesion, which are mere stepping stones toward durable plastics and easy access to purified drinking water.

\section{Outline of this thesis}

In order to elucidate the mechanism behind the laccase-mediated functionalisation of poly(ethersulfone) with phenolic oligomers, solution-phase oligomerisation of these phenols had to be characterised first. It is therefore that Chapter $\mathbf{2}$ describes how laccase converts 4-hydroxybenzoic acid only slowly, while it rapidly oligomerises the resulting dimers. The initially formed dimers are the result of C3-C3', C3-O and C1-C3' coupling, after which their oligomerisation results in a multitude of minimally abundant products.

As described in Chapter 3, these findings allowed for the rational design of novel monomeric positively charged phenolics. Laccase-mediated conversion of these monomers, additional membrane cleaning experiments and the use of PES model compounds were used to elucidate the mechanism behind the laccase-mediated modification of poly(ethersulfone). Being required to use multiple tools in order to be able to clarify the functionalisation mechanism proved how challenging it is to do so. We anticipated that this was not merely true in our case, but would have proven difficult within this scientific field more often. It is therefore that Chapter 4 reviews recent work regarding laccasemediated surface functionalisation, and critically evaluates this work with respect to the likelihood of (the intended) laccase-mediated grafting. This revealed multiple cases in which grafting proved highly improbable.

In order to extend the "toolbox" for achieving mild (copper-mediated) polymer functionalisation, Chapter 5 describes Fenton-like oxidation and subsequent reduction by $\mathrm{NaBH}_{4}$ to generate hydroxy moieties on a poly (methyl methacrylate) surface. The presence of these chemical anchors allowed for 
subsequent surface silanisation using $\mathrm{SiHCl}_{3}$ to result in a nano-structured superhydrophobic layer. Finally, we investigated the options for further decorating this layer with fluorinated alkenes and alkynes through hydrosilylation involving the $\mathrm{Si}-\mathrm{H}$ moieties present on the nanostructure.

Chapter 6 completes this thesis with a general discussion regarding the work presented herein. A summary of the highlights of each individual chapter will be complemented by the presentation of alternative research avenues and future prospects.

\section{References}

[1] A. Brownsell, "Chemistry's image problem", can be found under https://www.chemistryworld.com/opinion/chemistrys-image-problem/3008806.article, 2018.

[2] F. Rosi, C. Miliani, R. Braun, R. Harig, D. Sali, B. G. Brunetti, A. Sgamellotti, Angew. Chem. Int. Ed. 2013, 52, 5258-5261.

[3] C.-H. Kuo, Y. Tang, L.-Y. Chou, B. T. Sneed, C. N. Brodsky, Z. Zhao, C.-K. Tsung, J. Am. Chem. Soc. 2012, 134, 14345-14348.

[4] L. Scheres, M. Giesbers, H. Zuilhof, Langmuir 2010, 26, 4790-4795.

[5] A. Taglietti, C. R. Arciola, A. D'Agostino, G. Dacarro, L. Montanaro, D. Campoccia, L. Cucca, M. Vercellino, A. Poggi, P. Pallavicini, L. Visai, Biomaterials 2014, 35, 1779-1788.

[6] G. Siqueira, J. Bras, A. Dufresne, Langmuir 2010, 26, 402-411.

[7] S. Alwarappan, C. Liu, A. Kumar, C.-Z. Li, J. Phys. Chem. C2010, 114, 12920-12924.

[8] Y. T. Sul, B. S. Kang, C. J ohansson, H. S. Um, C. J. Park, T. Albrektsson, J. Biomed. Mater. Res., Part A 2009, 89A, 942-950.

[9] A. Tiraferri, C. D. Vecitis, M. Elimelech, ACS Appl. Mater. Interfaces 2011, 3, 2869-2877.

[10] S. A. Yao, R. E. Ruther, L. Zhang, R. A. Franking, R. J. Hamers, J. F. Berry, J. Am. Chem. Soc. 2012, 134, 15632-15635.

[11] K. Ellinas, S. P. Pujari, D. A. Dragatogiannis, C. A. Charitidis, A. Tserepi, H. Zuilhof, E. Gogolides, ACS Appl. Mater. Interfaces 2014, 6, 6510-6524.

[12] R. A. Dickie, Prog. Org. Coat. 1994, 25, 3-22.

[13] P. Muller in Pure Appl. Chem., Vol. 66, 1994, p. 1077.

[14] A. I. Rusanov, Surf. Sci. Rep. 1996, 23, 173-247.

[15] R. Sen, D. Gahtory, J. Escorihuela, J. Firet, S. P. Pujari, H. Zuilhof, Chem. Eur. J. 2017, 23, 13015-13022.

[16] E. Yousif, R. Haddad, SpringerPlus 2013, 2, 398.

[17] U. Ali, K. J. B. A. Karim, N. A. Buang, Polym. Rev. 2015, 55, 678-705.

[18] H. Liu, J. Huang, Z. Chen, G. Chen, K.-Q. Zhang, S. S. Al-Deyab, Y. Lai, Chem. Eng. J. 2017, 330, 26-35.

[19] a) H. Flemming, Appl. Microbiol. Biotechnol. 2002, 59, 629-640; b) N. Nady, M. C. R. Franssen, H. Zuilhof, M. S. Mohyeldin, R. Boom, K. Schroën, Desalination 2011, 275, 1-9; c) T. Nguyen, F. Roddick, L. Fan, Membranes 2012, 2, 804-840; d) R. Zhang, Y. Liu, M. He, Y. Su, X. Zhao, M. Elimelech, Z. Jiang, Chem. Soc. Rev. 2016, 45, 5888-5924.

[20] S. Nishimoto, B. Bhushan, RSC Adv. 2013, 3, 671-690.

[21] J. Zhang, J. Li, Y. Han, Macromol. Rapid Commun. 2004, 25, 1105-1108.

[22] J. C. Vickerman, I. S. Gilmore, Surface analysis: the principal techniques, 2nd ed., Wiley, Chichester, 2009.

[23] D. Quéré, Annu. Rev. Mater. Res. 2008, 38, 71-99.

[24] W. Barthlott, C. Neinhuis, Planta 1997, 202, 1-8.

[25] a) P. Patel, C. K. Choi, D. D. Meng, JALA: Journal of the Association for Laboratory Automation 2010, 15, 114-119; b) L. A. Goetz, B. Jalvo, R. Rosal, A. P. Mathew, J. Membr. Sci. 2016, 510, 238-248. 
[26] R. J. Green, J. Davies, M. C. Davies, C. J. Roberts, S. J. B. Tendler, Biomaterials 1997, 18, 405-413.

[27] a) Y.-H. Zhao, K.-H. Wee, R. Bai, J. Membr. Sci. 2010, 362, 326-333; b) G. Kang, M. Liu, B. Lin, Y. Cao, Q. Yuan, Polymer 2007, 48, 1165-1170; c) Z. Cao, S. Jiang, Nano Today 2012, 7, 404-413; d) A. Papra, N. Gadegaard, N. B. Larsen, Langmuir 2001, 17, 1457-1460.

[28] D. Hetemi, J. Pinson, Chem. Soc. Rev. 2017, 46, 5701-5713.

[29] J. Wencel-Delord, F. Glorius, Nat. Chem. 2013, 5, 369-375.

[30] a) L. Bárdos, H. Baránková, Thin Solid Films 2010, 518, 6705-6713; b) R. Morent, N. D. Geyter, T. Desmet, P. Dubruel, C. Leys, Plasma Processes Polym. 2011, 8, 171-190; c) D. P. Dowling, I. S. Miller, M. Ardhaoui, W. M. Gallagher, J. Biomater. Appl. 2011, 26, 327-347.

[31] J. F. Friedrich, S. Geng, W. Unger, A. Lippitz, J. Erdmann, H. V. Gorsler, C. Wöll, A. Schertel, K. Bierbaum, Surf. Coat. Technol. 1995, 74-75, 664-669.

[32] R. A. N. Pertile, F. K. Andrade, C. Alves, M. Gama, Carbohydr. Polym. 2010, 82, 692-698.

[33] F. Khelifa, S. Ershov, Y. Habibi, R. Snyders, P. Dubois, Chem. Rev. 2016, 116, 3975-4005.

[34] a) S. E. Mallakpour, A.-R. Hajipour, A.-R. Mahdavian, A. Zadhoush, F. Ali-Hosseini, Eur. Polym. J. 2001, 37, 1199-1206; b) J. A. Barish, J. M. Goddard, J. Food Sci. 2011, 76, E586-E591; c) G. J. Price, A. A. Clifton, F. Keen, Polymer 1996, 37, 5825-5829.

[35] J. A. Barish, J. M. Goddard, J. Appl. Polym. Sci. 2011, 120, 2863-2871.

[36] R. R. Carvalho, S. P. Pujari, E. X. Vrouwe, H. Zuilhof, ACS Appl. Mater. Interfaces 2017, 9, 16644-16650.

[37] a) M. Homayoonfal, A. Akbari, M. R. Mehrnia, Desalination 2010, 263, 217-225; b) A. Rahimpour, Desalination 2011, 265, 93-101; c) P. S. Zhong, N. Widjojo, T.-S. Chung, M. Weber, C. Maletzko, J. Membr. Sci. 2012, 417-418, 52-60.

[38] a) S. Gam-Derouich, M. Gosecka, S. Lepinay, M. Turmine, B. Carbonnier, T. Basinska, S. Slomkowski, M.-C. Millot, A. Othmane, D. Ben Hassen-Chehimi, M. M. Chehimi, Langmuir 2011, 27, 9285-9294; b) S. Mahouche-Chergui, S. Gam-Derouich, C. Mangeney, M. M. Chehimi, Chem. Soc. Rev. 2011, 40, 4143-4166.

[39] a) J. V. Gulmine, P. R. Janissek, H. M. Heise, L. Akcelrud, Polym. Degrad. Stab. 2003, 79, 385-397; b) O. Chiantore, M. Lazzari, Polymer 2001, 42, 17-27; c) A. Torikai, M. Ohno, K. Fueki, J. Appl. Polym. Sci. 1990, 41, 1023-1032; d) M. Zeuner, J. Meichsner, H. U. Poll, Plasma Sources Sci. Technol. 1995, 4, 406-415.

[40] a) E. Aracri, A. Fillat, J. F. Colom, A. Gutiérrez, J. C. del Río, Á. T. Martínez, T. Vidal, Bioresour. Technol. 2010, 101, 8211-8216; b) P. Widsten, C. Heathcote, A. Kandelbauer, G. M. Guebitz, G. S. Nyanhongo, E. Nugroho Prasetyo, T. Kudanga, Process Biochem. 2010, 45, 1072-1081.

[41] a) N. Nady, K. Schroën, M. C. R. Franssen, B. van Lagen, S. Murali, R. M. Boom, M. S. Mohyeldin, H. Zuilhof, ACS Appl. Mater. Interfaces 2011, 3, 801-810; b) T. Kudanga, G. S. Nyanhongo, G. M. Guebitz, S. Burton, Enzyme Microb. Technol. 2011, 48, 195-208.

[42] B. Leader, Q. J. Baca, D. E. Golan, Nat. Rev. Drug Discovery 2008, 7, 21-39.

[43] A. Sellami-Kamoun, A. Haddar, N. E.-H. Ali, B. Ghorbel-Frikha, S. Kanoun, M. Nasri, Microbiol. Res. 2008, 163, 299-306.

[44] C. Fadda, A. M. Sanguinetti, A. D. Caro, C. Collar, A. Piga, Compr. Rev. Food Sci. Food Saf. 2014, 13, 473-492.

[45] a) M. C. R. Franssen, P. Steunenberg, E. L. Scott, H. Zuilhof, J. P. M. Sanders, Chem. Soc. Rev. 2013, 42, 6491-6533; b) R. DiCosimo, J. McAuliffe, A. J. Poulose, G. Bohlmann, Chem. Soc. Rev. 2013, 42, 6437-6474; c) R. Fernandez-Lafuente, J. Mol. Catal. B 2010, 62, 197-212.

[46] a) P. J. Strong, H. Claus, Crit. Rev. Environ. Sci. Technol. 2011, 41, 373-434; b) M. Fernández-Fernández, M. Á. Sanromán, D. Moldes, Biotechnol. Adv. 2013, 31, 1808-1825.

[47] M. Alcalde in Industrial Enzymes: Structure, Function and Applications (Eds.: J. Polaina, A. P. MacCabe), Springer Netherlands, Dordrecht, 2007, pp. 461-476. 
[48] a) M. R. Coy, T. Z. Salem, J. S. Denton, E. S. Kovaleva, Z. Liu, D. S. Barber, J. H. Campbell, D. C. Davis, G. W. Buchman, D. G. Boucias, M. E. Scharf, Insect Biochem. Mol. Biol. 2010, 40, 723-732; b) N. Santhanam, J. M. Vivanco, S. R. Decker, K. F. Reardon, Trends Biotechnol. 2011, 29, 480-489; c) H. Yoshida, J. Chem. Soc. Trans. 1883, 43, 472-486.

[49] a) P. Baldrian, FEMS Microbiol. Rev. 2006, 30, 215-242; b) S. V. S. Kumar, P. S. Phale, S. Durani, P. P. Wangikar, Biotechnol. Bioeng. 2003, 83, 386-394; c) C. M. Rivera-Hoyos, E. D. Morales-Álvarez, R. A. Poutou-Piñales, A. M. Pedroza-Rodríguez, R. RodrÍguez-Vázquez, J. M. Delgado-Boada, Fungal Biol. Rev. 2013, 27, 67-82.

[50] J. M. M. Verkade, L. J. C. van Hemert, P. J. L. M. Quaedflieg, H. E. Schoemaker, M. Schürmann, F. L. van Delft, F. P. J. T. Rutjes, Adv. Synth. Catal. 2007, 349, 1332-1336.

[51] S. M. Jones, E. I. Solomon, Cell. Mol. Life Sci. 2015, 72, 869-883.

[52] K. Piontek, M. Antorini, T. Choinowski, J. Biol. Chem. 2002, 277, 37663-37669.

[53] D. L. Johnson, J. L. Thompson, S. M. Brinkmann, K. A. Schuller, L. L. Martin, Biochemistry 2003, 42, 10229-10237.

[54] T. Kudanga, E. Nugroho Prasetyo, J. Sipilä, A. Eberl, G. S. Nyanhongo, G. M. Guebitz, J. Mol. Catal. B 2009, 61, 143-149.

[55] S. Slagman, J. Escorihuela, H. Zuilhof, M. C. R. Franssen, RSC Adv. 2016, 6, 99367-99375.

[56] Ú. Fillat, D. I barra, M. Eugenio, A. Moreno, E. Tomás-Pejó, R. Martín-Sampedro, Fermentation 2017, 3, 17.

[57] L. Munk, A. K. Sitarz, D. C. Kalyani, J. D. Mikkelsen, A. S. Meyer, Biotechnol. Adv. 2015, 33, 13-24.

[58] R. Chandra Rajak, R. Banerjee, RSC Adv. 2016, 6, 61301-61311.

[59] L. J. Jönsson, E. Palmqvist, N.-O. Nilvebrant, B. Hahn-Hägerdal, Appl. Microbiol. Biotechnol. 1998, 49, 691-697.

[60] D. M. Mate, M. Alcalde, Microb. Biotechnol. 2016, 10, 1457-1467.

[61] L. Lloret, G. Eibes, T. A. Lú-Chau, M. T. Moreira, G. Feijoo, J. M. Lema, Biochem. Eng. J. 2010, 51, 124-131.

[62] Y. Dai, L. Yin, J. Niu, Environ. Sci. Technol. 2011, 45, 10611-10618.

[63] R. Khlifi, L. Belbahri, S. Woodward, M. Ellouz, A. Dhouib, S. Sayadi, T. Mechichi, J. Hazard. Mater. 2010, 175, 802-808.

[64] a) S. Camarero, O. García, T. Vidal, J. Colom, J. C. del Río, A. Gutiérrez, J. M. Gras, R. Monje, M. J. Martínez, Á. T. Martínez, Enzyme Microb. Technol. 2004, 35, 113-120; b) S. Camarero, D. Ibarra, Á. T. Martínez, J. Romero, A. Gutiérrez, J. C. del Río, Enzyme Microb. Technol. 2007, 40, 1264-1271; c) M. Montazer, A. Sadeghian Maryan, Appl. Biochem. Biotechnol. 2010, 160, 2114-2128.

[65] J. Polak, A. Jarosz-Wilkolazka, Process Biochem. 2012, 47, 1295-1307.

[66] J.-R. Jeon, E.-J. Kim, K. Murugesan, H.-K. Park, Y.-M. Kim, J.-H. Kwon, W.-G. Kim, J.-Y. Lee, Y.-S. Chang, Microb. Biotechnol. 2010, 3, 324-335.

[67] a) K. W. Wellington, N. I. Kolesnikova, Bioorg. Med. Chem. 2012, 20, 4472-4481; b) T. Qwebani-Ogunleye, N. I. Kolesnikova, P. Steenkamp, C. B. de Koning, D. Brady, K. W. Wellington, Bioorg. Med. Chem. 2017, 25, 1172-1182.

[68] O. E. Adelakun, T. Kudanga, A. Parker, I. R. Green, M. le Roes-Hill, S. G. Burton, J. Mol. Catal. B 2012, 74, 29-35.

[69] M. Marzorati, B. Danieli, D. Haltrich, S. Riva, Green Chem. 2005, 7, 310-315.

[70] T. Kudanga, E. Nugroho Prasetyo, P. Widsten, A. Kandelbauer, S. J ury, C. Heathcote, J. Sipilä, H. Weber, G. S. Nyanhongo, G. M. Guebitz, Bioresour. Technol. 2010, 101, 2793-2799.

[71] a) E. Aracri, C. Díaz Blanco, T. Tzanov, Green Chem. 2014, 16, 2597-2603; b) Y. Yu, Q. Wang, J. Yuan, X. Fan, P. Wang, L. Cui, Carbohydr. Polym. 2016, 137, 549-555.

[72] M. Božič, S. Gorgieva, V. Kokol, Carbohydr. Polym. 2012, 89, 854-864.

[73] K. M. G. Hossain, M. D. González, G. R. Lozano, T. Tzanov, J. Biotechnol. 2009, 141, 58-63. 
[74] W. Jia, Q. Wang, X. Fan, A. Dong, Y. Yu, P. Wang, RSC Adv. 2017, 7, 12977-12983.

[75] N. Nady, K. Schroën, M. C. R. Franssen, R. Fokkink, M. S. Mohyeldin, H. Zuilhof, R. M. Boom, J. Colloid Interface Sci. 2012, 378, 191-200. 


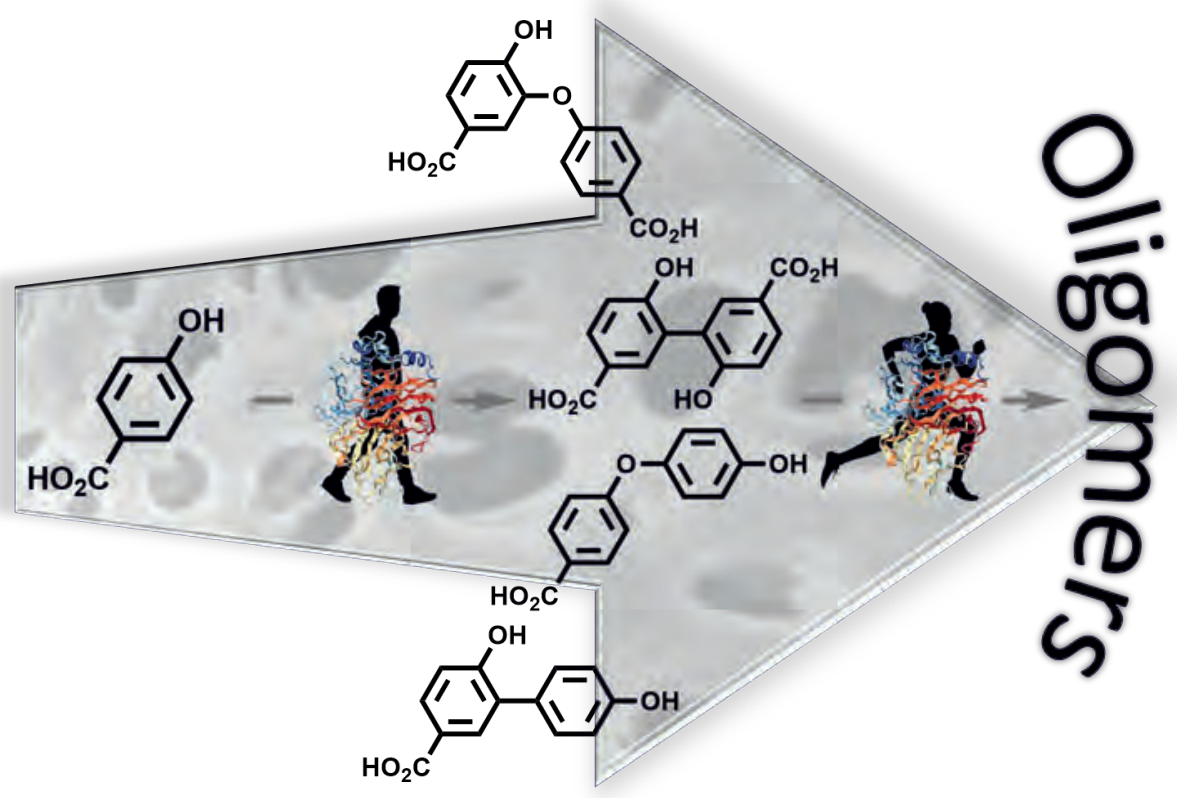




\title{
Chapter 2
}

\section{Characterisation of the laccase-mediated oligomerisation of 4-hydroxybenzoic acid}

\begin{abstract}
Modifying inert poly(ethersulfone) membranes using laccase has proven to be an environmentally benign and easily applicable process to alter the membrane's surface properties. By this method phenolic acid monomers such as 4-hydroxybenzoic acid form an anti-fouling overlayer on the membrane surface. In order to enhance the anti-fouling capabilities even further, it is important to study the molecular details of this reaction. However, the products from laccase-mediated modification, either on a surface or in solution, have been studied only poorly. Here we report on the formation of C3-C3', C3-O and C1-C3' linked dimers as the first products of the solution-phase laccasemediated oligomerisation of 4-hydroxybenzoic acid. These dimers can also act as substrate for laccase, and we show that their enzymatic oxidative coupling occurs far more rapidly than that of 4hydroxybenzoic acid, which indicates that they are highly reactive intermediates that are efficiently polymerised onwards. The reactivity of each dimer is of large influence on its yield; dimers that are converted more rapidly are less abundant. This knowledge allows for further improvement of applications involving laccase, such as the grafting of phenols on surfaces and enzymatic polymerisation of lignin fragments.
\end{abstract}

This chapter has been adapted from:

Characterization of the laccase-mediated oligomerization of 4-hydroxybenzoic acid

S. Slagman, J. Escorihuela, H. Zuilhof, M. C. R. Franssen, RSC Adv. 2016, 6, 99367-99375

* This chapter is directly followed by its corresponding supporting information. In order to access NMR spectra, the reader is referred to the online version of the publication mentioned above. 


\section{I ntroduction}

Laccases are copper-containing oxidoreductases found in plants, bacteria, insects and fungi. In nature they act on catechols (1,2-dihydroxybenzenes) and other phenols, and convert these to radical species via radical cation intermediates. ${ }^{[1]}$ Most members of the laccase family exhibit a very large substrate scope, which makes them useful catalysts in organic chemistry. ${ }^{[2]}$ This scope is enlarged even further when mediator systems are employed. ${ }^{[3]}$ In these cases a mediator molecule is first oxidised by laccase, after which it oxidises a molecule that cannot directly be oxidised by laccase. This makes laccases interesting biocatalysts and underlines clearly why they are used in a wide variety of applications ranging from polymerisation ${ }^{[4]}$ and small-molecule synthesis ${ }^{[5]}$ to waste water purification $^{[6]}$ and the modification of biorenewables. ${ }^{[7]}$ Within the sub-class of small-molecule synthesis the formation of dimers out of (naturally occurring) phenols draws special attention. ${ }^{[8]}$

For some of these transformations the molecular structure of the product(s) is studied, mainly when it concerns single product reactions or polymerisation reactions. ${ }^{[9]}$ However in many cases a particular transformation is only characterised by a qualitative description of observed changes in the system; for example a shift in the UV spectrum or disappearance of the starting material according to mass spectrometry. This is common when dye or drug degradation is studied. [10]

Apart from the previously mentioned wide variety of applications for this enzyme, laccase is also known for its ability to graft small monomers from (natural) fibres such as wood or cotton. ${ }^{[11]}$ Within our group we developed a methodology to widen the scope of grafting even further by modifying poly(ethersulfone) (PES) membranes. ${ }^{[12]}$ In order to alter the surface of the membrane, 4hydroxybenzoic acid was grafted from the membrane via a laccase-mediated reaction. Through this environmentally benign process 4-hydroxybenzoic acid and a range of analogues thereof could be covalently attached to the otherwise rather inert membrane. This method can be used to change the surface properties of such membranes in a tailor-made fashion, e.g. making it anti-fouling. ${ }^{[12 d]}$ However, for further optimisation of the anti-fouling properties, a study of the molecular details of the grafting process is essential.

Although some studies have looked at the reaction products resulting from the laccase-mediated conversion of vanillic acid (4-hydroxy-3-methoxybenzoic acid) and oligomerisation reactions of syringic acid (4-hydroxy-3,5-dimethoxybenzoic acid), further studies into the product profile for laccase-

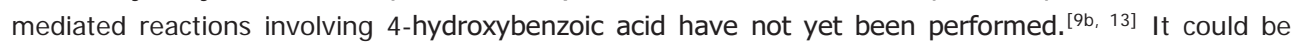
that, in the case of vanillic and syringic acid, the additional methoxy substituents block positions on the benzene ring that would otherwise be available for $\mathrm{C}-\mathrm{C}$ and $\mathrm{C}-\mathrm{O}$ bond formation, which thereby makes the product profile for these substrates less complex and easier to study. Considering its importance as monomer in membrane modification we aimed to study the laccase-mediated oligomerisation of 4-hydroxybenzoic acid in solution and provide detailed insight in the product profile of this reaction. In order to do so, the reaction products were separated by analytical or preparative HPLC and subsequently analysed by mass spectrometry. The two major products were also analysed by NMR and their structure was proven through independent synthesis. Studies regarding the reactivity of these products were conducted subsequently.

\section{Results and discussion}

Trametes versicolor laccase is able to oxidise phenolic substrates to their radical cations, which rapidly lose a proton to form radical species that further react to oligomers under ambient conditions at $\mathrm{pH}$ 5. The reaction conditions were adopted from previous work related to the mentioned modification of PES membranes and employed in our current studies of the solution phase reaction of 4-hydroxybenzoic acid (4-HBA) and laccase. ${ }^{[12 c]}$ In the current study the buffer solution was changed from a sodium acetate/acetic acid buffer to an ammonium acetate/acetic acid buffer in order to be compatible with MS analysis. The reaction mixture of laccase and 4-HBA was mildly agitated for $24 \mathrm{~h}$ at room temperature using ambient air as oxidant. Thereafter laccase was removed from the mixture 
by centrifugal filtration through an ultrafiltration membrane to obtain enzyme-free material ready for LC-MS analysis.

\section{Determination of 4-hydroxybenzoic acid conversion}

Earlier attempts to polymerise 4-hydroxybenzoic acid (4-HBA) were reported to be unsuccessful, ${ }^{\text {[9b] }}$ and it was claimed that 4-hydroxybenzoic acid was not oxidised under the used conditions at all (either of three different laccases in a mixed acetone/buffer medium). However, by employing, among others, colourimetric studies, it was made clear that 4-hydroxybenzoic acid could be oxidised by laccase. ${ }^{[12 c]}$ Further research on neither the conversion nor the products from the laccase-mediated oxidation of 4HBA have been reported.

In order to determine the amount of 4-HBA converted by the enzyme we measured the UV absorption of 4-hydroxybenzoic acid at $254 \mathrm{~nm}$ at several known concentrations. Using this calibration curve (SI Figure S1) we determined the conversion by diluting an aliquot of the reaction mixture and measuring UV absorption at $254 \mathrm{~nm}$. From this we concluded that $26 \%$ of the starting material was converted after $24 \mathrm{~h}$ using $26.8 \mathrm{mM}$ 4-hydroxybenzoic acid and $1.0 \mathrm{U} \mathrm{ml}^{-1}$ of laccase.

\section{Characterisation of the main products of the laccase-mediated oligomerisation of 4- hydroxybenzoic acid}

The follow-up goal was to determine the molecular structure of the main products of laccasemediated oligomerisation of 4-hydroxybenzoic acid. By employing LC-MS analysis we aimed to retrieve information on the molecular mass of the respective products and thereby an indication of their structure and a rough estimate of the relative abundance of the products. This information allows for a pre-selection of the most important molecules for further studies.

Separation of the main products of laccase-mediated oligomerisation of 4-hydroxybenzoic acid was achieved on a C18 column through a two-step isocratic elution with an acidified water/acetonitrile mixture. As was already anticipated, the reaction of 4-hydroxybenzoic acid with laccase resulted in a complex mixture of products (Figure 1); more than 25 individual peaks are visible in the LC-UV chromatogram (254 nm).

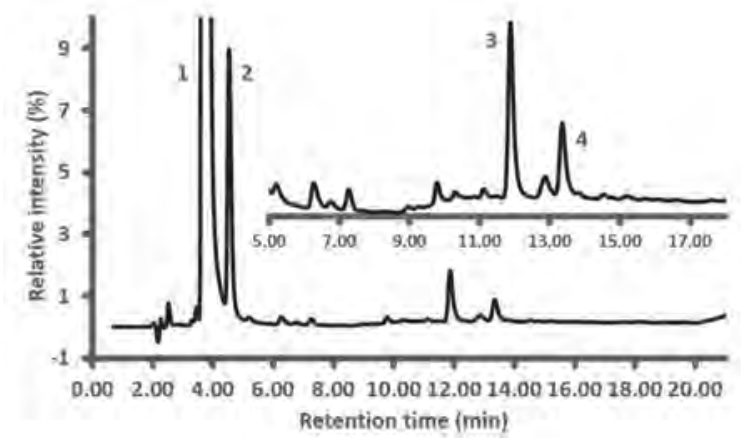

Figure 1. LC-UV chromatogram ( $254 \mathrm{~nm}$ ) after the reaction of 4-hydroxybenzoic acid (peak at $3.7 \mathrm{~min}$ ) and laccase. Insert: Scale-out of the chromatogram for the section from 5.0 to $18.0 \mathrm{~min}$. Reaction conditions: 4-hydroxybenzoic

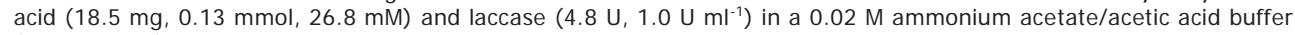
$(\mathrm{pH} 5,5.0 \mathrm{ml})$ mildly agitated at room temperature for $24 \mathrm{~h}$. Separation was achieved through LC-method: LC1.

Table 1. Peaks in the LC-UV chromatogram (Figure 1) and the corresponding molecular ion.

\begin{tabular}{|c|c|c|c|c|}
\hline \# & RT $(\min )^{[a]}$ & {$[M-H]^{-}(D a)^{[b]}$} & Chemical formula & Theoretical [M - H] $]^{-}$(Da) \\
\hline 1 & 3.7 & 137.02311 & $\mathrm{C}_{7} \mathrm{H}_{5} \mathrm{O}_{3}$ & 137.02442 \\
\hline 2 & 4.5 & 273.03978 & $\mathrm{C}_{14} \mathrm{H}_{9} \mathrm{O}_{6}$ & 273.04046 \\
\hline 3 & 11.8 & 273.03971 & $\mathrm{C}_{14} \mathrm{H}_{9} \mathrm{O}_{6}$ & 273.04046 \\
\hline 4 & 13.3 & 379.04394 & $\mathrm{C}_{20} \mathrm{H}_{11} \mathrm{O}_{8}$ & 379.04594 \\
\hline
\end{tabular}

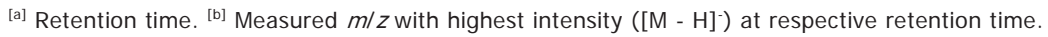


A selection of the most important products was made based on the highest peaks in the chromatogram (Table 1). For all four components listed in Table 1 the corresponding molecular ion [M - $\mathrm{H}]^{-}$was the signal with the highest intensity: adducts with other ions only made a minor contribution (see SI Figures S6-S12 for respective mass spectra and extracted ion chromatograms). Peak 1 at retention time $3.7 \mathrm{~min}$ corresponds to the starting material 4-hydroxybenzoic acid. The two product peaks with the highest UV absorption (peak 2 and 3 at retention time $4.5 \mathrm{~min}$ and $11.8 \mathrm{~min}$ ), both correspond to a molecule with a mass of $274 \mathrm{Da}$. Furthermore, the third most intense product peak 4 has a retention time of $13.3 \mathrm{~min}$ and corresponds to a compound having a molecular mass of $380 \mathrm{Da}$. It is well known that laccase is able to oligomerise phenols and it is therefore logical that the products formed first are dimers that in our case would have a mass of $\left.274 \mathrm{Da} .{ }^{[9 a},{ }^{13}\right]$ These two expected dimers are most likely formed through either a carbon-centred radical or an oxygen-centred radical, following a mechanism which is based on the proposed mechanism for other phenol dimerisations (Scheme 1). ${ }^{[9 a}{ }^{13]}$ Laccase generates a 4-hydroxybenzoic acid radical cation that easily loses a proton. The thus formed radical is delocalised over the phenolic system. Two of these radicals can recombine following tautomerisation to form dimer $\mathbf{1}$ or $\mathbf{2}$ directly (top route). Alternatively, a second molecule of 4-hydroxybenzoic acid attacks one of these radicals to form another radical (propagation), from which a hydrogen atom is abstracted (termination) to form either dimer $\mathbf{1}$ or $\mathbf{2}$ (bottom route).

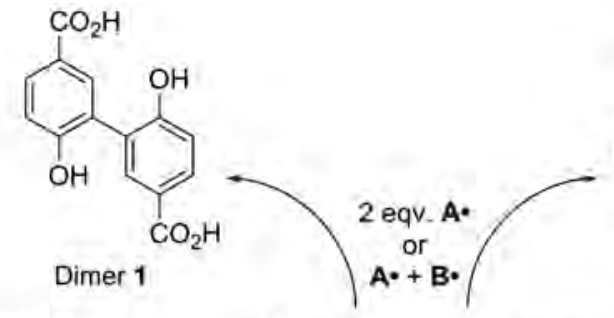<smiles>O=C(O)c1ccc(Oc2cc(C(=O)O)ccc2O)cc1</smiles>

Dimer 2

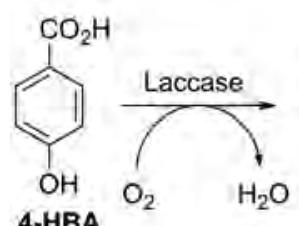<smiles>O=C(O)c1ccc(O)cc1</smiles>

4-HBA

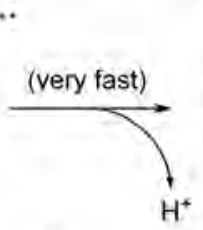<smiles>COc1ccc(C(=O)O)cc1</smiles><smiles>COC(=O)c1ccc(C(=O)O)cc1</smiles>

A.<smiles>CCCCCCCC(=O)OCc1ccc(C(=O)O)cc1</smiles><smiles>O=C(O)c1ccc(O)c(-c2cc(C(=O)O)ccc2O)c1</smiles>

Dimer 1

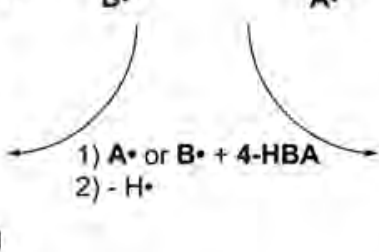

1) $A \cdot H \cdot$ or<smiles>O=C(O)c1ccc(Oc2cc(C(=O)O)ccc2O)cc1</smiles>

Dimer 2

Scheme 1. Proposed pathway for formation dimer 1 and 2. Top: Recombination of two 4-HBA radicals, bottom: propagation followed by $\mathrm{H} \cdot$ abstraction.

Independent synthesis of these dimers would facilitate their identification. The C3-C3'-coupled dimer 1 could be synthesised through an Ullmann coupling with two equivalents of methyl 3-iodo-4methoxy-benzoate in the presence of copper bronze (Scheme 2). ${ }^{[14]}$ In order to obtain reasonable yields the reaction has to be heated above $250^{\circ} \mathrm{C}$ and stirred vigorously in a shallow boiling tube. This process yielded $68 \%$ of methylated compound $\mathbf{6}$. Demethylation was thereafter achieved through the use of boron tribromide followed by continuous extraction of the very polar product dimer $\mathbf{1} .{ }^{[15]}$

The C3-O-coupled product $\mathbf{9}$ could be generated through a Chan-Lam coupling between commercially available methyl isovanillate and 4-methoxycarbonylphenylboronic acid in the presence of molecular sieves. ${ }^{[16]}$ Again, demethylation through the use of boron tribromide and continuous 
extraction resulted in the desired dimer. ${ }^{[15]}$ In order to properly compare HPLC retention times at $24 \mathrm{~h}$, a 4-hydroxybenzoic acid reaction sample was spiked with both synthesised dimers $\mathbf{1}$ and $\mathbf{2}$ and subsequently eluted under the same conditions as a non-spiked sample. The results (SI Figure S17) make clear that the retention time for both dimers corresponds to the retention time of the putative dimers as reported in Table 1 (entry 2 and 3). Furthermore, the mass spectra of the chemically synthesised dimers correspond to those of the dimers that are generated enzymatically (SI Figures S9, $\mathrm{S} 10, \mathrm{~S} 20$ and S21).

2<smiles>COc1ccc(C(C)=O)cc1I</smiles>

5<smiles>COc1ccc(C(C)=O)cc1-c1cc(C(C)=O)ccc1OC</smiles>

6. $68 \%$<smiles>O=C(O)c1ccc(O)c(-c2ccccc2O)c1</smiles>

Dimer $1,6 \%$<smiles>COc1ccc(C(C)=O)cc1Oc1ccc(C(=O)OCC(C)=O)cc1Oc1cc(Oc2ccc([B]O)cc2)c(OC)c(C(C)=O)c1</smiles>

Scheme 2. Synthesis route for top: dimer $\mathbf{1}$ and bottom: dimer $\mathbf{2}$.

Definitive structure elucidation of the two putative dimers was achieved through their isolation from the reaction mixture by means of preparative HPLC. A comparison of ${ }^{1} \mathrm{H}$ NMR data (SI Table S2) of both the chemically synthesised as well as the enzymatically generated material shows that the putative structures indeed correspond to the two main products of laccase-mediated conversion of 4hydroxybenzoic acid.

In order to obtain an even more complete characterisation of the products originating from the laccase-mediated oxidation of 4-hydroxybenzoic acid we also aimed to obtain information on the structure of minor products. The third most intense UV product peak corresponds to a compound with $m / z=379$ (Table 1, entry 4). After analysing the corresponding putative chemical formula, we concluded that this is most probably a trimeric benzoquinone (a non-exhaustive list of possible trimeric benzoquinone species is depicted in Scheme 3) in which one of the sub-units is decarboxylated, eventually leading to a 1,2- or 1,4-benzoquinone. Formation of a benzoquinone is further supported by the occurrence of a second peak in the mass spectrum of this product at $m / z=381$ (SI Figures S11 and S12). This additional peak arises because the benzoquinone moiety is easily reduced, which is apparent in the formation of a diol moiety two mass units heavier than its corresponding benzoquinone. This reduction can take place at the spray tip of the electrospray ionisation source of the mass spectrometer in negative ionisation mode. Similar reduction processes have been observed for 1,2-benzoquinone and have been extensively reviewed. ${ }^{[17]}$<smiles>O=C1C=C(Oc2ccc(C(=O)O)cc2)C(=O)C(Oc2ccc(C(=O)O)cc2)=C1</smiles>

10<smiles>O=C1C=CC(=O)C(Oc2ccc(C(=O)O)cc2-c2cc(C(=O)O)ccc2O)=C1</smiles>

11<smiles>O=C1C=CC(Oc2ccc(C(=O)O)cc2-c2cc(C(=O)O)ccc2O)=CC1=O</smiles>

12

Scheme 3. Possible structures for a benzoquinone trimer. 
The formation of a benzoquinone is in line with previous findings involving vanillic acid as substrate, for which several benzoquinone-containing products were isolated. ${ }^{[9 a}$, 13] However, mechanistic details on this process remain until this point unclear. Where there are only limited options to couple two monomers, there is a multitude of options to couple three.

MS-MS analysis did not provide enough information to suggest a useful lead compound and more structural NMR-based information was required. To obtain the material in sufficient quantities to be analysed by NMR the laccase-mediated modification of 4-hydroxybenzoic acid was performed at 250 $\mathrm{ml}$ scale. Separation and purification of the individual components, with the isolation of one or more trimeric benzoquinones as main goal, would then be achieved through repetitive preparative HPLCruns. However, 40 repetitive separations still did not provide enough material for proper NMR characterisation. The trimeric benzoquinones appear to be only very minor constituents of the complex product mixture.

As we were unsuccessful with regards to isolating the suspected trimeric benzoquinone we did not try to isolate other minor products, since we expect those to be even less abundant.

\section{Determination of conversion towards dimers 1 and 2}

In a fashion similar to the determination of the conversion of 4-hydroxybenzoic acid, we were able to determine the yield of dimers $\mathbf{1}$ and $\mathbf{2}$ after laccase-mediated oligomerisation of 4-HBA. After obtaining enough of both dimers through chemical synthesis as described above, we plotted a calibration curve (SI Figures S2 and S3) for the UV absorption of the corresponding peaks after LCseparation at several known concentrations. By interpolation of the matching peak area of a $24 \mathrm{~h}$ laccase/4-HBA reaction sample to the calibration curve we determined that dimer $\mathbf{1}$ was obtained in $2 \%$ yield and dimer $\mathbf{2}$ in $0.2 \%$ yield. This means that the majority (almost $24 \%$ ) of the previously mentioned $26 \%$ conversion of 4 -HBA (over $24 \mathrm{~h}$ ) corresponds to other products. This most likely represents conversion to a fast array of unique higher order oligomeric products which are all present in minute amounts. This composition therefore actually supports the efficient polymerisation of 4-HBA and its success in membrane modification.

\section{Laccase-mediated transformation of dimers 1 and 2}

The low abundance of the two dimers in the reaction mixture prompted us to further investigate the role of these products in the reaction pathway. We hypothesised that these were not final products, but merely intermediates in a reaction mixture that is in a steady state in which the dimers are rapidly converted to different products, and in fact significantly faster than monomeric 4-HBA.

To test this hypothesis, we determined the conversion of both dimers under conditions similar to those of the reaction of laccase and 4-hydroxybenzoic acid. In this case the solubility of the dimers turned out to be the limiting factor, as a result we lowered the concentration of the starting dimer to $3.40 \mathrm{mM}$ and changed the reaction medium to a $10 \%$ solution of methanol in $0.02 \mathrm{M}$ ammonium acetate/acetic acid buffer ( $\mathrm{pH}$ 5). Dimer conversion was determined under five different conditions in which either one of the two dimers was solely present as substrate in the reaction mixture, or together with the second dimer, or in a mixture with also 4-hydroxybenzoic acid present (Table 2).

Table 2. Laccase-mediated conversion of dimers $\mathbf{1}$ and $\mathbf{2}$ after $24 \mathrm{~h}$.

\begin{tabular}{cccc}
\hline$\#$ & Substrates & Conversion dimer 1 & Conversion dimer 2 \\
1 & Dimer 1 & $48 \%$ & - \\
2 & Dimer 2 & - & $77 \%$ \\
3 & Dimer 1 + dimer 2 & $53 \%$ & $69 \%$ \\
4 & Dimer 1 + 4-HBA & $55 \%$ & - \\
5 & Dimer 2 + 4-HBA & - & $69 \%$ \\
\hline
\end{tabular}

Dimer(s) (1.4 mg, $5.0 \mu \mathrm{mol}, 3.40 \mathrm{mM}$ ) and/or 4-hydroxybenzoic acid (4-HBA) (entry 4 and 5 only, $0.7 \mathrm{mg}, 5.0$ $\mu \mathrm{mol}, 3.40 \mathrm{mM})$ were reacted in the presence of laccase $\left(0.51 \mathrm{U} \mathrm{ml}^{-1}\right)$ in a mixture of methanol and a $0.02 \mathrm{M}$ ammonium acetate/acetic acid buffer (1:9) at room temperature and $\mathrm{pH} 5$ for $24 \mathrm{~h}$. 
As shown in Table 2, conversion of both dimers is similar across all screened conditions. Around $50 \%$ of dimer $\mathbf{1}$, and around $70-75 \%$ of dimer $\mathbf{2}$ is converted under these conditions. This is significantly higher than the conversion of 4-hydroxybenzoic acid after $24 \mathrm{~h}$, even under such nonoptimal reaction conditions (lower concentration starting material and methanol as co-solvent). This supports the hypothesis that these dimers are highly reactive intermediate products. Furthermore, the higher conversion of dimer $\mathbf{2}$ might also, in part, explain the lower yield of this dimer in laccasemediated oligomerisation of 4-hydroxybenzoic acid, as it is more rapidly converted than dimer $\mathbf{1}$.

In order to validate the hypothesis that dimers $\mathbf{1}$ and $\mathbf{2}$ are more reactive than the monomer 4HBA, we performed DFT calculations on the conversion of these substrates to their radical cations (SI Table S1). As depicted in Scheme 1, this is the rate-limiting step and will thereby likely govern the reactivity of the substrates.

We found that generation of the 4-hydroxybenzoic acid radical cation in water occurs less easily than that of either dimer (energy gap of $154.9 \mathrm{kcal} \mathrm{mol}^{-1}$ (4-HBA) vs $152.7 \mathrm{kcal} \mathrm{mol}^{-1}$ (dimer 1) and $147.8 \mathrm{kcal} \mathrm{mol}^{-1}$ (dimer 2)). These results support our hypothesis that dimers $\mathbf{1}$ and $\mathbf{2}$ are highly reactive intermediates in the laccase-mediated conversion of 4-hydroxybenzoic acid.

Apart from some expected dimerisation in the laccase-mediated treatment of dimer $\mathbf{2}$ towards a molecule with a molecular mass of $546 \mathrm{Da}$ (SI Figures S13 and S14) little information was obtained on the nature of the formed products from dimer conversion. In the condition where dimer $\mathbf{1}$ and dimer $\mathbf{2}$ were both present as a substrate, two more peaks (with very low intensity) corresponding to a molecule with molecular mass 546 Da were observed (data not shown). Again (as with the conversion of 4-HBA) a wide range of molecules seem to be formed, albeit all in very low yields.

\section{The role of less abundant products}

As indicated by the rapid conversion of dimers $\mathbf{1}$ and $\mathbf{2}$, products with low abundance might still be important in the oligomerisation process. This implies that the pre-selection based on peak intensity will not include all relevant compounds. To find out what other molecules might have been formed, a closer look was taken into the mechanism of dimer formation (Scheme 1).

So far, the contributions of radical $\mathbf{A}^{\boldsymbol{\bullet}}$ and $\mathbf{B}^{\boldsymbol{\bullet}}$ to form dimers $\mathbf{1}$ and $\mathbf{2}$ have been considered. Participation of radical $\mathbf{C}^{\bullet}$ might, however, also give rise to dimers. In this case dimers $\mathbf{3}$ and $\mathbf{4}$ would be formed by attack of a molecule of $4-\mathrm{HBA}$, another radical $\mathbf{A}^{\bullet}$ or radical $\mathbf{B}^{\bullet}$ on radical $\mathbf{C}^{\bullet}$ (Scheme 4 ). Similar as to the identification of dimers $\mathbf{1}$ and $\mathbf{2}$, we now aimed to obtain and detect possible dimers $\mathbf{3}$ and $\mathbf{4}$ to find out if these also play a role in the effective oligomerisation of 4-HBA by laccase.

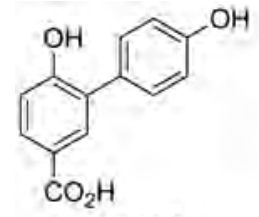

Dimer 3

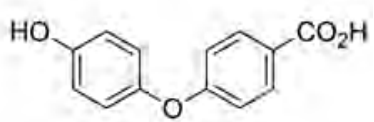

Dimer 4

Scheme 4. Dimers possibly originating from radical $\mathbf{C}^{\bullet}(230 \mathrm{Da})$.

These dimers will most likely have a stronger affinity for the used C18 column during LCseparation, therefore the LC-method was slightly adjusted towards a higher percentage of the less polar solvent acetonitrile (LC3). Indeed, several peaks that correspond to a molecule having a mass of 230 Da were observed (SI Figure S15). Some of these peaks represent MS-generated adducts or fragments of molecules which are present in far higher concentrations, i.e. the peak at 10.6 min, which represents a fragment of dimer $\mathbf{2}$. The peak at 7.7 min (mass spectrum in SI Figure S16), however, might correspond to one of the putative dimers.

In order to test this hypothesis, we followed a similar approach as to the identification of dimers $\mathbf{1}$ and 2. For this purpose, chemically synthesised dimers need to be obtained in order to compare their retention times with those corresponding to the putative dimeric structures. Due to the even lower 
apparent abundance (based on UV intensity) of the putative dimers $\mathbf{3}$ and $\mathbf{4}$ compared to that of the possible benzoquinone (i.e. compound $\mathbf{1 0}, \mathbf{1 1}$ or $\mathbf{1 2}$ ), isolation through preparative HPLC was not pursued.

Whereas dimer $\mathbf{4}$ is commercially available, dimer $\mathbf{3}$ was synthesised using a slight modification of a recently published one-pot two-step synthesis involving a Suzuki coupling followed by saponification (Scheme 5). ${ }^{[18]}$<smiles>CC(=O)c1ccc(O)c(I)c1</smiles>

13<smiles>COc1ccc(O)cc1</smiles>

14

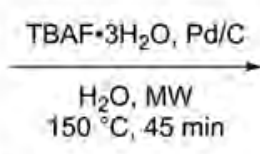

$150^{\circ} \mathrm{C}, 45 \mathrm{~min}$

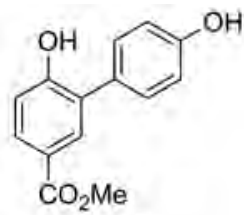

15

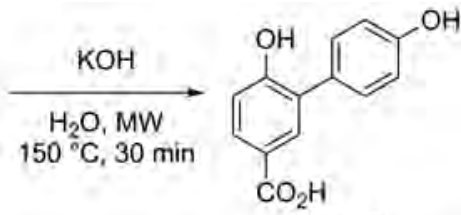

Dimer 3

$13 \%$ over two steps

Scheme 5. Synthesis route for dimer 3.

Co-injection of the dimers with an aliquot from the reaction of 4-HBA with laccase indicated the presence of dimer $\mathbf{3}$ (SI Figure S18). Dimer 4, however, was difficult to observe (SI Figure S19). Over the course of the reaction the yield of dimer 3 fluctuated between approximately $0.01 \%$ and $0.02 \%$ (SI Figure S23). The fact that these yields are so low indicates rapid oligomerisation of both dimers.

To test this hypothesis, as was done before for dimers $\mathbf{1}$ and $\mathbf{2}$, the laccase-mediated conversion of dimers $\mathbf{3}$ and $\mathbf{4}$ was monitored after $\mathbf{2 4} \mathrm{h}$. Yet again the solubility, especially of dimer $\mathbf{4}$, proved to be an issue. This was overcome by increasing the percentage of the co-solvent methanol in the reaction mixture to $18.5 \%$. As shown in Table 3 the conversion of dimer 3 went to completion over the course of $24 \mathrm{~h}$ (entry 1 and 3). This was also true for dimer 4 when it was present as sole substrate (entry 2). However, when 4-hydroxybenzoic acid was also present, only $70 \%$ of dimer $\mathbf{4}$ was converted (entry 4).

Full conversion of dimer $\mathbf{3}$ through laccase-mediated oligomerisation explains the finding that the yield of this dimer, resulting from the laccase-mediated oligomerisation of 4-HBA, is so low. This is, most likely, also true for dimer $\mathbf{4}$ when one takes into account the even less optimal conditions (18.5\% methanol) under which these reactions have been performed.

Table 3. Laccase-mediated conversions of dimers $\mathbf{3}$ and $\mathbf{4}$ after $24 \mathrm{~h}$.

\begin{tabular}{cccc}
\hline$\#$ & Substrates & Conversion dimer 3 & Conversion dimer 4 ${ }^{\text {[a] }}$ \\
1 & Dimer 3 & $>99 \%$ & - \\
2 & Dimer 4 & - & $99 \%$ \\
3 & Dimer 3 + 4-HBA & $>99 \%$ & - \\
4 & Dimer 4 + 4-HBA & - & $70 \%$ \\
\hline
\end{tabular}

Dimer 3 or 4 (3.40 mM) and 4-hydroxybenzoic acid (4-HBA) (entry 3 and 4 only, $3.40 \mathrm{mM}$ ) were reacted in the presence of laccase $\left(0.51 \mathrm{U} \mathrm{ml}^{-1}\right)$ in a mixture of methanol and a $0.02 \mathrm{M}$ ammonium acetate/acetic acid buffer $(1: 4.4)$ at room temperature and $\mathrm{pH} 5$ for $24 \mathrm{~h}$. ${ }^{\text {a] }}$ The determined conversions of dimer 4 are an average of 3 measurements.

Based solely on the conversion at $24 \mathrm{~h}$, it is not possible to differentiate between the rates for the reaction of laccase with dimer $\mathbf{3}$ or $\mathbf{4}$. The conversion of both dimers, with or without 4-HBA, was therefore also monitored over time. The conversion of dimer $\mathbf{3}$ occurred with similar rates in both the presence and absence of 4-HBA, and is already almost complete at $4 \mathrm{~h}$ (SI Figure S24). In the absence of 4-HBA, dimer 4 seems to be converted slightly slower than dimer 3 (SI Figure S25). When 4hydroxybenzoic acid is present, however, the reaction does not seem to obey regular kinetics. The reason for this is unclear. Considering the structural similarity between both, it could be that 4-HBA acts as a competitive inhibitor for the conversion of dimer 4.

The relative reactivities of each dimer were also estimated by calculating the energy required for the formation of the radical cation from the respective dimer (SI Table S1). These results (dimer 3: $137.1 \mathrm{kcal} \mathrm{mol}^{-1}$; dimer $\mathbf{4}$ : $138.1 \mathrm{kcal} \mathrm{mol}^{-1}$ ) clearly underline that dimers $\mathbf{3}$ and $\mathbf{4}$ are even more 
reactive than dimers $\mathbf{1}$ and $\mathbf{2}$ and thus than 4-HBA. Apart from this, the subtle differences in calculated relative energies are reflected in the rate of reaction of each dimer (dimer $\mathbf{3}>$ dimer $\mathbf{4}>>$ dimer $\mathbf{2}>$ > dimer $\mathbf{1}>>$ 4-HBA).

These results indicate that the yield of dimers is largely governed by rapid subsequent oligomerisation. Furthermore, both the theoretical calculations and the experimental evidence thus support our hypothesis that the formed dimers are not final products, but merely intermediates in the laccase-mediated conversion of 4-hydroxybenzoic acid.

\section{Conclusions}

Laccase-mediated oligomerisation of 4-hydroxybenzoic acid results in a wide variety of products. The moderately reactive monomer 4-HBA is first converted to at least three distinct dimers in which the monomers are bound via either a C3-C3', C3-O or C1-C3' linkage. By providing both theoretical as well as experimental evidence we showed that these dimers were converted far more rapidly than 4HBA itself. This is reflected in the yield of the above-mentioned dimers: the more reactive, the lower the yield. These results indicate that the dimers are not final products of the reaction of 4hydroxybenzoic acid with laccase, but highly reactive intermediates in the efficient formation of polymers.

The potential for covalent modification of poly(ethersulfone) membranes by such polymers clearly highlights the importance of these findings. We expect that the newly obtained knowledge will open up avenues towards discovering new, and improving current, applications in the laccase-mediated modification of phenols and other lignin fragments.

\section{Experimental}

\section{Chemicals and general remarks}

4-Hydroxybenzoic acid (>99\%), methyl 4-hydroxy-3-iodobenzoate (97\%), palladium on carbon (10 wt\%), potassium hydroxide ( $\geq 85 \%)$ and laccase from Trametes versicolor (EC number: 1.10.3.2) were obtained from Sigma-Aldrich. Methyl 3-iodo-4-methoxybenzoate (98\%), copper acetate (98\%), copper bronze powder (99\%) came from Alfa Aesar. Methyl 3-hydroxy-4-methoxybenzoate (>98\%) and 4-(4-hydroxyphenoxy)benzoic acid (>99\%) were obtained from TCl Chemicals. 4Methoxycarbonylphenylboronic acid (97\%) was purchased from Fluorochem, ABTS diammonium salt (98\%) was obtained from Amresco, boron tribromide (>99\%), 4-hydroxyphenylboronic acid (97\%) were obtained from Acros Organics, tetrabutylammonium fluoride trihydrate (for synthesis) was purchased from Merck Millipore, glacial acetic acid (ULC/MS) was obtained from Biosolve and ammonium acetate (analytical grade) was obtained from Fisher Scientific. Reactions were performed under ambient conditions unless stated otherwise. Reactions heated by microwave irradiation were performed in an Anton-Paar Monowave 400 microwave with internal temperature control. Purification by flash chromatography was conducted by using flash silica gel $60 .{ }^{1} \mathrm{H}$ NMR and ${ }^{13} \mathrm{C}$ NMR spectra were recorded on a $400 \mathrm{MHz}$ Bruker Avance spectrometer. All signals were referenced relative to the residual solvent signal and coupling constants $(/)$ are given in $\mathrm{Hz}$. High resolution mass spectra were recorded on a Thermo Scientific Exactive instrument. All MS chromatograms were normalised to the peak with highest intensity. Extracted ion chromatograms are always displayed as a plot of $[\mathrm{M}-\mathrm{H}]^{-} \pm$ $0.50 \mathrm{Da}$.

\section{Enzyme assay}

The specific activity of commercially available laccase from Trametes versicolor (EC number: 1.10.3.2) was determined spectroscopically on a Varian Cary 50 Scan UV/Vis spectrometer by measuring the increase of absorbance for the oxidation of ABTS to the ABTS radical cation ( $\varepsilon=$ 36000). ABTS diammonium salt was dissolved in $0.02 \mathrm{M}$ ammonium acetate/acetic acid buffer ( $\mathrm{pH} 5$, 
$3.5 \mathrm{ml}$ ) and laccase was added after which the increase of absorbance at $\lambda=420 \mathrm{~nm}$ was followed for $1.0 \mathrm{~min}$. The specific activity was determined to be $3.2 \mathrm{U} \mathrm{mg}^{-1}$ laccase. ${ }^{[19]}$

\section{Laccase-mediated conversion of 4-hydroxybenzoic acid}

4-Hydroxybenzoic acid (18.5 mg, $0.13 \mathrm{mmol}, 26.8 \mathrm{mM}$ ) and laccase from Trametes versicolor (EC number: 1.10.3.2, $4.8 \mathrm{U}, 1.0 \mathrm{U} \mathrm{ml}^{-1}$ ) were dissolved in $0.02 \mathrm{M}$ ammonium acetate/acetic acid buffer $(\mathrm{pH} 5,5.0 \mathrm{ml})$ and mildly agitated at room temperature for $24 \mathrm{~h}$. Laccase was thereafter removed from the reaction mixture using a $9 \mathrm{kDa}$ protein concentrator by employing centrifugal force (Hermle $Z$ 206A). The supernatant was then either diluted in buffer and subjected to LC-MS analysis, or its constituents were separated by employing preparative HPLC.

\section{Laccase-mediated conversion of dimers}

Dimers ( $3.40 \mathrm{mM}$ each) were dissolved in methanol (10\% for dimers $\mathbf{1}$ and $\mathbf{2}$, or $\mathbf{1 8 . 5 \%}$ for dimers 3 and 4) and mixed with either $0.02 \mathrm{M}$ ammonium acetate/acetic acid buffer ( $\mathrm{pH}$ 5) or a solution of 4hydroxybenzoic acid $(3.40 \mathrm{mM}$ ) in $0.02 \mathrm{M}$ ammonium acetate/acetic acid buffer ( $\mathrm{pH}$ 5). To this, a solution of laccase from Trametes versicolor $\left(0.51 \mathrm{U} \mathrm{ml}^{-1}\right)$ in buffer was added after which the reaction mixture was mildly agitated at room temperature. Subsequent removal of laccase by employing centrifugal force (10 kDa protein concentrator, Hermle Z 206A) was followed by appropriately diluting the supernatant with buffer, after which the mixture was subjected to LC-MS analysis.

\section{Analytical LC-MS setup}

For liquid chromatography/mass spectrometry (LC-MS) a Thermo Finnigan Surveyor MS pump was coupled to a photodiode array detector (Thermo Finnigan Surveyor PDA) and either one of the two following: a mass spectrometer (Finnigan LXQ) or high-resolution mass spectrometer (Thermo Scientific Exactive), and both instruments were equipped with an electrospray ionisation source. Measurements were conducted in negative ionisation mode. LC-MS-MS measurements were conducted with the Finnigan LXQ mass spectrometer, where components were fragmented at a normalised collision energy of $35 \mathrm{eV}$. Separation was achieved through an Altima HP C18 column $(2.1 \mathrm{~mm} \times 100$ $\mathrm{mm}, 3 \mu \mathrm{m}$; Grace) at room temperature and a flow rate of $0.2 \mathrm{ml} \mathrm{min}^{-1}$. The mobile phases consisted of $0.1 \%$ formic acid in water (mobile phase A) and in acetonitrile (mobile phase B).

The used method for separation of the products of 4-hydroxybenzoic acid oligomerisation was as follows: isocratic elution for $5.0 \mathrm{~min}$ at $15 \%$ B followed by isocratic elution for $12.0 \mathrm{~min}$ at $20 \% \mathrm{~B}$ after which a steep gradient towards $100 \%$ B (within $0.1 \mathrm{~min}$ ) was followed by isocratic elution for $13.0 \mathrm{~min}$ at $100 \%$ B. This was followed by a steep gradient back to $15 \%$ B and the system was then reequilibrated by employing 15.0 min of $15 \%$ B (LC1).

The used method for separation of the products of the laccase-mediated transformation of dimers $\mathbf{1}$ and $\mathbf{2}$ was as follows: gradient elution starting at 15\% B which increases linearly to $50 \%$ B over the course of $20.0 \mathrm{~min}$. This was followed by eluting with $100 \%$ B for 10.0 min after which the system was re-equilibrated by employing $15 \%$ B for 15.0 min (LC2).

The used method for detection of decarboxylated products from the laccase-mediated conversion of 4-HBA was as follows: isocratic elution for $5.0 \mathrm{~min}$ at $15 \% \mathrm{~B}$, directly followed by a linear increase of $20 \%$ B to $40 \%$ B over the course of $12.0 \mathrm{~min}$. This was followed by eluting with $100 \%$ B for 6.0 or $15.0 \mathrm{~min}$ (dependent on the dilution of the injected sample) after which the system was reequilibrated by employing $15 \%$ B for 13.0 min (LC3).

\section{Preparative HPLC-setup}

Preparative HPLC-separations were performed on an Alltima C18 column ( $22 \mathrm{~mm} \times 250 \mathrm{~mm}$, 5 $\mu \mathrm{m}$; Grace). A LC-8A Shimadzu LC-pump was connected to a SPD-M10AVP Shimadzu diode array detector equipped with a FRC-10A Shimadzu fraction collector. Separation was achieved at room 
temperature at a constant flow of $22 \mathrm{ml} \mathrm{min}^{-1}$ using a mixture of $0.1 \%$ formic acid in water (mobile phase A) and $0.1 \%$ formic acid in acetonitrile (mobile phase B). The used method was as follows: isocratic elution for $17.0 \mathrm{~min}$ at $22.5 \% \mathrm{~B}$ followed by an $11.0 \mathrm{~min}$ rinsing step of $100 \% \mathrm{~B}$ to finish with re-equilibration at $22.5 \% \mathrm{~B}$.

\section{Quantitative NMR analysis}

The purity of the synthesised dimers was assessed using quantitative NMR analysis. Either of the dimers and the internal standard (3,5-dinitrobenzoic acid) were dissolved in $0.6 \mathrm{ml}$ deuterated methanol (dimer $\mathbf{1}$ ) or deuterated acetone (dimers $\mathbf{2}$ and $\mathbf{3}$ ). ${ }^{1} \mathrm{H}$ NMR spectra were thereafter recorded with high relaxation delay $\mathrm{d} 1$ (10 seconds). The purity of the synthesised dimer could then be determined according to the formula: percentage purity by weight $=W_{i d} W_{s} * A_{d} A_{i s} * M W_{s} M W_{i s} *$ $H_{i d} H_{s}$. In this formula $W$ stands for the weighed amount of internal standard/dimer; $A$ corresponds to the area of the particular NMR peak, MW stands for the molecular weight of the internal standard/dimer and $H$ stands for the number of protons represented by the integral of the NMR peak.

\section{Determination of conversion}

The peak area for the signal corresponding to the molecule of interest (UV, $254 \mathrm{~nm}$ ) was determined after eluting samples with a known concentration of the specific target compound at the conditions as stated for the analytical LC-MS setup. This peak area was determined at several known concentrations (see SI Figures S1-S5 for details). The peak area at $0.00 \mu \mathrm{M}$ was subtracted from the other data points and a line was fitted through the points using the least squares method (calibration curve). The obtained values for the slope $(\boldsymbol{a})$ and offset $(\boldsymbol{b})$ were used in the formula with form $\boldsymbol{y}=$ $\boldsymbol{a} \boldsymbol{x}+\boldsymbol{b}$, where $\boldsymbol{x}$ is the concentration of the specific target compound, and $\boldsymbol{y}$ is the measured peak area.

To determine the unknown concentration of the compound of interest, after the reaction had finished, an aliquot of the reaction mixture was diluted appropriately in order for the concentration to be interpolated on the calibration curve. This diluted aliquot was eluted under the same conditions as described above and the peak area for the UV peak ( $254 \mathrm{~nm}$ ) corresponding to the target compound was determined. This obtained $y$ was then used to regressively calculate the concentration of the compound of interest and thereby also the conversion of starting material.

\section{Synthetic procedures}<smiles>COC(=O)c1ccc(OC)c(-c2cc(C(=O)OC)ccc2OC)c1</smiles>
$2.2 \mathrm{~Hz}, 2 \mathrm{H}), 6.99(\mathrm{~d}, 3 /(\mathrm{H}, \mathrm{H})=8.7 \mathrm{~Hz}, 2 \mathrm{H}), 3.88(\mathrm{~s}, 6 \mathrm{H}), 3.82(\mathrm{~s}, 6 \mathrm{H}) .{ }^{13} \mathrm{C} \mathrm{NMR}\left(101 \mathrm{MHz}, \mathrm{CDCl}_{3}\right): \delta=$ 167.0, 161.0, 133.1, 131.4, 126.9, 122.4, 110.5, 56.0, 52.1; HRMS (ESI) $m / z[M+N a]^{+} 353.0992$ (calcd. 353.0996).<smiles>COc1ccc(C(C)=O)cc1Oc1ccc(C(C)=O)cc1</smiles>

Methyl 4-methoxy-3-(4-(methoxycarbonyl) phenoxy) benzoate (9). Methyl 3-hydroxy-4-methoxybenzoate (200 mg, $1.10 \mathrm{mmol}, 1$ eq.) was dissolved in dry dichloromethane $(20 \mathrm{ml})$. (4(Methoxycarbonyl)phenyl)boronic acid (108 mg, $0.59 \mathrm{mmol}, 0.55$ eq.), copper acetate (109 mg, $0.59 \mathrm{mmol}, 0.55$ eq.), pyridine (48 $\mu \mathrm{l}, 0.59$ 
mmol, 0.55 eq.) and 20 molecular sieves ( $4 \AA$ ) were added and the mixture was stirred at room temperature under minor pressure from an air filled balloon. ${ }^{[16]}$ When, according to TLC, all (4(methoxycarbonyl)phenyl)boronic acid was consumed, another portion of (4(methoxycarbonyl)phenyl)boronic acid, copper acetate and pyridine was added and the reaction was stirred until all methyl 3-hydroxy-4-methoxybenzoate was consumed. Hereafter water $(10 \mathrm{ml})$ was added and the product was extracted using dichloromethane. The organic layer was then concentrated in vacuo and the product was purified by silica gel chromatography (petroleum ether 40:60/ethyl acetate $4: 1$ ) to yield the product as a white solid (170 mg, $0.54 \mathrm{mmol}, 49 \%)$. ${ }^{1} \mathrm{H}$ NMR (400 MHz, $\left.\mathrm{CDCl}_{3}\right): \delta=8.01-7.94(\mathrm{~m}, 2 \mathrm{H}), 7.92(\mathrm{dd}, 3 /(\mathrm{H}, \mathrm{H})=8.6,2.1 \mathrm{~Hz}, 1 \mathrm{H}), 7.73(\mathrm{~d}, 3 /(\mathrm{H}, \mathrm{H})=2.1 \mathrm{~Hz}, 1 \mathrm{H})$, $7.03(\mathrm{~d}, 3 /(\mathrm{H}, \mathrm{H})=8.7 \mathrm{~Hz}, 1 \mathrm{H}), 6.94-6.84(\mathrm{~m}, 2 \mathrm{H}), 3.87(\mathrm{~s}, 3 \mathrm{H}), 3.85(\mathrm{~s}, 3 \mathrm{H}), 3.83(\mathrm{~s}, 3 \mathrm{H}) .{ }^{13} \mathrm{C} N M R$ $\left(101 \mathrm{MHz}, \mathrm{CDCl}_{3}\right): \delta=166.6,166.1,161.7,155.5,143.0,131.6,128.1,124.3,123.4,123.3,115.9$, 112.05, 56.0, 52.1, 52.0; HRMS (ESI) $m / z$ [M + Na] 339.0831 (calcd. 339.0839).

General procedure for demethylation. A solution of the methylated precursor ( $0.6 \mathrm{mmol}, 1$ eq. $)$ in dichloromethane $(5 \mathrm{ml})$ was loaded in a three-necked flask on which an acid gas trap was fixed..$^{[15]}$ The solution was stirred under $\mathrm{N}_{2}$ and cooled to $-30{ }^{\circ} \mathrm{C}$ after which $\mathrm{BBr}_{3}(1.2 \mathrm{ml}, 12.8 \mathrm{mmol}, 20$ eq.) was slowly added. Thereafter the mixture was further stirred for $18 \mathrm{~h}$ during which the mixture was allowed to warm up to room temperature. The reaction was quenched by carefully adding cold water $(1 \mathrm{ml})$. The mixture was concentrated in vacuo after which it was continuously extracted using ethyl acetate $(80 \mathrm{ml})$. The obtained solid was further purified using either silica gel chromatography or reversed phase preparative HPLC.<smiles>O=C(O)c1ccc(O)c(-c2cc(C(=O)O)ccc2O)c1</smiles>

Dimer 1

3,3'-bi(4-Hydroxybenzoic acid) (dimer 1). Dimer 1 was synthesised according to the general procedure for demethylation and purified through preparative HPLC (isocratic elution at $20 \%$ acetonitrile in water $+0.1 \%$ formic acid) to yield dimer $\mathbf{1}$ as an off-white solid ( $6 \mathrm{mg}, 0.02 \mathrm{mmol}^{*}, 6 \% *$, purity: $86 \%$ (q-NMR)). ${ }^{1} \mathrm{H}$ NMR (400 $\mathrm{MHz}, \mathrm{MeOD}): \delta=7.94-7.87(\mathrm{~m}, 4 \mathrm{H}), 6.96(\mathrm{~d}, 3 /(\mathrm{H}, \mathrm{H})=9.1 \mathrm{~Hz}, 2 \mathrm{H}) .{ }^{13} \mathrm{C} \mathrm{NMR}$ (101 MHz, MeOD): $\delta=170.0,160.5,135.0,132.1,126.4,122.9$, 116.6; HRMS (ESI ) $m / z$ [M - H] 273.0406 (calcd. 273.0405). * Based on 86\% purity material<smiles>O=C(O)c1ccc(Oc2cc(C(=O)O)ccc2O)cc1</smiles>

Dimer 2

3-(4-Carboxyphenoxy)-4-hydroxybenzoic acid (dimer 2). Dimer 2 was synthesised according to the general procedure for demethylation and purified by silica gel chromatography (petroleum ether 40:60/ethyl acetate/glacial acetic acid 1:5:0.02) to yield dimer $\mathbf{2}$ as an off-white solid (179 mg, 0.60 mmol*, 99\%*, purity: 93\% (q-NMR)). ${ }^{1} \mathrm{H}$ NMR (400 MHz, acetone- $\left.d_{6}\right): \delta=8.03(\mathrm{~d}, 3 /(\mathrm{H}, \mathrm{H})=8.8 \mathrm{~Hz}, 2 \mathrm{H}), 7.85(\mathrm{dd}, 3 /(\mathrm{H}, \mathrm{H})=8.5$, $2.0 \mathrm{~Hz}, 1 \mathrm{H}), 7.70(\mathrm{~d}, 3 /(\mathrm{H}, \mathrm{H})=2.0 \mathrm{~Hz}, 1 \mathrm{H}), 7.16(\mathrm{~d}, 3 /(\mathrm{H}, \mathrm{H})=8.5 \mathrm{~Hz}, 1 \mathrm{H})$, $7.02(\mathrm{~d}, 3 /(\mathrm{H}, \mathrm{H})=8.8 \mathrm{~Hz}, 2 \mathrm{H}) .{ }^{13} \mathrm{C}$ NMR $\left(101 \mathrm{MHz}\right.$, Acetone- $\left.\alpha_{6}\right): \delta=167.1,166.8,162.6,154.6$, 142.7, 132.6, 129.1, 125.8, 124.4, 123.9, 118.0, 117.0; HRMS (ESI) $\mathrm{m} / z$ [M - H] 273.0405 (calcd. 273.0405). * Based on $93 \%$ purity material<smiles>O=C(O)c1ccc(O)c(-c2ccc(O)cc2)c1</smiles>

Dimer 3

4',6-Dihydroxy-[1,1'-biphenyl]-3-carboxylic acid (dimer 3). Tetrabutylammonium fluoride ( $372 \mathrm{mg}, 1.18 \mathrm{mmol}, 4.0$ eq.) was dissolved in water $(12 \mathrm{ml})$ in a $30 \mathrm{ml}$ microwave vessel. Thereafter, methyl 4-hydroxy-3iodobenzoate ( $83 \mathrm{mg}, 0.30 \mathrm{mmol}, 1$ eq.), 4-hydroxyphenylboronic acid (53 mg, $0.38 \mathrm{mmol}, 1.3$ eq.) and $10 \mathrm{wt} \% \mathrm{Pd} / \mathrm{C}(5.9 \mathrm{mg}, 6.0 \mu \mathrm{mol}, 2.0 \mathrm{~mol} \%)$ were added. ${ }^{[18]}$ The suspension was sonicated for $5 \mathrm{~min}$ and rapidly transferred into the microwave, where it was stirred for $45 \mathrm{~min}$ at $150{ }^{\circ} \mathrm{C}$. After cooling to room temperature, potassium hydroxide $(500 \mathrm{mg})$ was added and the mixture was stirred for another 30 min at $150{ }^{\circ} \mathrm{C}$ under microwave irradiation. After 10 min sonication to break down lumps, the mixture 
was extracted with methyl tert-butylether and concentrated in vacuo. Silica gel chromatography (heptane/EtOAc/AcOH 3:2:0.1\% to 1:1:0.1\%) afforded the product as blue/white solid (9 $\mathrm{mg}, 0.04$ mmol*, 13\%*, purity: 96\% (q-NMR)). ${ }^{1} \mathrm{H}$ NMR (400 MHz, Acetone- $\left.\alpha_{6}\right): \delta=9.02$ (br s, $\left.1 \mathrm{H}\right), 7.97$ (d, J $=2.2 \mathrm{~Hz}, 1 \mathrm{H}), 7.84(\mathrm{dd}, J=8.4,2.2 \mathrm{~Hz}, 1 \mathrm{H}), 7.46(\mathrm{dt}, J=8.8,2.9,2.1 \mathrm{~Hz}, 2 \mathrm{H}), 7.04(\mathrm{~d}, J=8.4 \mathrm{~Hz}$, $1 \mathrm{H}), 6.91(\mathrm{dt}, J=8.6,2.9,2.2 \mathrm{~Hz}, 2 \mathrm{H}) .{ }^{13} \mathrm{C} \mathrm{NMR}\left(101 \mathrm{MHz}\right.$, Acetone- $\left.\alpha_{6}\right): \delta=167.5,159.2,157.7$, 133.2, 131.3, 130.8, 129.8, 129.3, 123.2, 116.7, 115.9. HRMS (ESI) $m / z$ [M - H]- 229.0498 (calcd. 229.0506). *Based on $96 \%$ purity material

\section{Quantum chemical calculations}

Before performing theoretical calculations, an initial conformational search was executed in order to determine the conformer distribution for both dimers and 4-HBA using Spartan'14. Hereby a constraint free search was performed and the resulting structures were optimised using the MMFF force field. For all structures the conformer with the lowest energy was chosen for further studies at M11L level. These geometries were optimised using density functional theory (M11L with the 6$311+G(d, p)$ basis set) in water as solvent using Gaussian09, this geometrical optimisation was also applied to the radical cations. ${ }^{[20]}$ Additionally, optimisation of all geometries was also performed at the B3LYP and MIIL level in vacuum with similar results albeit with higher relative energies.

\section{Acknowledgements}

This project was funded by the NanoNextNL project 04A.01 "Biomimetic selective layers".

\section{References}

[1] V. Madhavi, S. S. Lele, Bioresources 2009, 4, 1694-1717.

[2] a) M. Mogharabi, M. A. Faramarzi, Adv. Synth. Catal. 2014, 356, 897-927; b) S. Riva, Trends Biotechnol. 2006, 24, 219-226; c) S. Witayakran, A. J. Ragauskas, Adv. Synth. Catal. 2009, 351, 1187-1209.

[3] R. Reiss, J. Ihssen, M. Richter, E. Eichhorn, B. Schilling, L. Thöny-Meyer, PLoS One 2013, 8, e65633.

[4] a) F. Hollmann, I. W. C. E. Arends, Polymers 2012, 4, 759-793; b) S. Kobayashi, A. Makino, Chem. Rev. 2009, 109, 5288-5353; c) Y. Zhang, X. Fan, Q. Wang, A. Cavaco-Paulo, RSC Adv. 2016, 6, 49272-49280.

[5] a) G. A. Kraus, A. Thite, F. Liu, Tetrahedron Lett. 2009, 50, 5303-5304; b) S. Hajdok, J. Conrad, H. Leutbecher, S. Strobel, T. Schleid, U. Beifuss, J. Org. Chem. 2009, 74, 7230-7237; c) J. Gonzalez-Sabin, N. Rios-Lombardia, I. Garcia, N. M. Vior, A. F. Brana, C. Mendez, J. A. Salas, F. Moris, Green Chem. 2016, 18, 989-994; d) E. Ricklefs, M. Girhard, K. Koschorreck, M. S. Smit, V. B. Urlacher, ChemCatChem 2015, 7, 1857-1864.

[6] a) P. Demarche, C. Junghanns, R. R. Nair, S. N. Agathos, Biotechnol. Adv. 2012, 30, 933-953; b) S. Dong, H. Xiao, Q. Huang, J. Zhang, L. Mao, S. Gao, Sci. Rep. 2016, 6, 21396; c) X. Zhang, M. Hua, L. Lv, B. Pan, Sci. Rep. 2015, 5, 8253.

[7] a) M. C. R. Franssen, P. Steunenberg, E. L. Scott, H. Zuilhof, J. P. M. Sanders, Chem. Soc. Rev. 2013, 42, 6491-6533; b) A. Rico, J. Rencoret, J. C. del Río, A. T. Martínez, A. Gutiérrez, Biotechnology for Biofuels 2014, 7, 1-14.

[8] a) S. Ncanana, L. Baratto, L. Roncaglia, S. Riva, S. G. Burton, Adv. Synth. Catal. 2007, 349, 1507-1513; b) O. E. Adelakun, T. Kudanga, A. Parker, I. R. Green, M. le Roes-Hill, S. G. Burton, J. Mol. Catal. B 2012, 74, 29-35; c) E. Beneventi, S. Conte, M. R. Cramarossa, S. Riva, L. Forti, Tetrahedron 2015, 71, 3052-3058.

[9] a) A. Leonowicz, R. U. Edgehill, J. M. Bollag, Arch. Microbiol. 1984, 137, 89-96; b) R. Ikeda, J. Sugihara, H. Uyama, S. Kobayashi, Polym. Int. 1998, 47, 295-301. 
[10] a) S. Camarero, D. Ibarra, M. J. Martínez, Á. T. Martínez, Appl. Environ. Microbiol. 2005, 71, 1775-1784; b) L. Lloret, G. Eibes, T. A. Lú-Chau, M. T. Moreira, G. Feijoo, J. M. Lema, Biochem. Eng. J. 2010, 51, 124-131.

[11] a) J. Rencoret, E. Aracri, A. Gutiérrez, J. C. del Río, A. L. Torres, T. Vidal, A. T. Martínez, Biochem. Eng. J. 2014, 86, 16-23; b) T. Kudanga, G. S. Nyanhongo, G. M. Guebitz, S. Burton, Enzyme Microb. Technol. 2011, 48, 195-208; c) R. P. Chandra, C. Felby, A. J. Ragauskas, J. Wood Chem. Technol. 2005, 24, 69-81.

[12] a) S. van der Veen, N. Nady, M. C. R. Franssen, H. Zuilhof, R. M. Boom, T. Abee, K. Schroën, J. Appl. Polym. Sci. 2015, 132, 41576; b) N. Nady, M. C. R. Franssen, H. Zuilhof, R. M. Boom, K. Schroën, Water 2012, 4, 932-943; c) N. Nady, K. Schroën, M. C. R. Franssen, M. S. Mohyeldin, H. Zuilhof, R. M. Boom, J. Membr. Sci. 2012, 394, 69-79; d) N. Nady, K. Schroën, M. C. R. Franssen, R. Fokkink, M. S. Mohyeldin, H. Zuilhof, R. M. Boom, J. Colloid Interface Sci. 2012, 378, 191-200; e) N. Nady, K. Schroën, M. C. R. Franssen, B. van Lagen, S. Murali, R. M. Boom, M. S. Mohyeldin, H. Zuilhof, ACS Appl. Mater. Interfaces 2011, 3, 801-810.

[13] J. Bollag, S. Liu, R. D. Minard, Soil Biol. Biochem 1982, 14, 157-163.

[14] D. Drochner, W. Hüttel, S. E. Bode, M. Müller, U. Karl, M. Nieger, W. Steglich, Eur. J. Org. Chem. 2007, 2007, 1749-1758.

[15] J. F. W. McOmie, M. L. Watts, D. E. West, Tetrahedron 1968, 24, 2289-2292.

[16] S. M. Johnson, S. Connelly, I. A. Wilson, J. W. Kelly, J. Med. Chem. 2008, 51, 6348-6358.

[17] a) N. B. Cech, C. G. Enke, Mass Spectrom. Rev. 2001, 20, 362-387; b) G. Albarran, W. Boggess, V. Rassolov, R. H. Schuler, J. Phys. Chem. A 2010, 114, 7470-7478.

[18] B. Schmidt, M. Riemer, Eur. J. Org. Chem. 2015, 2015, 3760-3766.

[19] F. Arnold, G. Georgiou, M. Alcalde, T. Bulter in Directed Enzyme Evolution, Vol. 230, Humana Press, 2003, pp. 193-201.

[20] M. J. Frisch, G. W. Trucks, H. B. Schlegel, G. E. Scuseria, M. A. Robb, J. R. Cheeseman, G. Scalmani, V. Barone, B. Mennucci, G. A. Petersson, H. Nakatsuji, M. Caricato, X. Li, H. P. Hratchian, A. F. Izmaylov, J. Bloino, G. Zheng, J. L. Sonnenberg, M. Hada, M. Ehara, K. Toyota, R. Fukuda, J. Hasegawa, M. Ishida, T. Nakajima, Y. Honda, O. Kitao, H. Nakai, T. Vreven, J. A. Montgomery J r., J. E. Peralta, F. Ogliaro, M. J. Bearpark, J. Heyd, E. N. Brothers, K. N. Kudin, V. N. Staroverov, R. Kobayashi, J. Normand, K. Raghavachari, A. P. Rendell, J. C. Burant, S. S. Iyengar, J. Tomasi, M. Cossi, N. Rega, N. J. Millam, M. Klene, J. E. Knox, J. B. Cross, V. Bakken, C. Adamo, J. Jaramillo, R. Gomperts, R. E. Stratmann, O. Yazyev, A. J. Austin, R. Cammi, C. Pomelli, J. W. Ochterski, R. L. Martin, K. Morokuma, V. G. Zakrzewski, G. A. Voth, P. Salvador, J. J. Dannenberg, S. Dapprich, A. D. Daniels, Ö. Farkas, J. B. Foresman, J. V. Ortiz, J. Cioslowski, D. J. Fox, Revision D.01 ed., Gaussian, Inc., Wallingford, CT, USA, 2009. 


\section{Supporting information corresponding to Chapter 2}

\section{Figure S1. Calibration curve for the determination of the conversion of 4-HBA.}

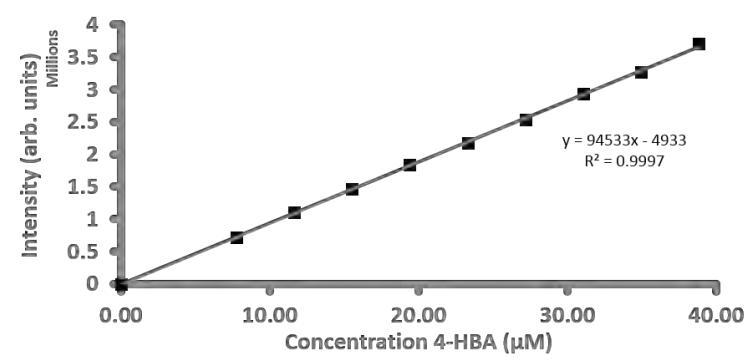

Measured peak areas (UV chromatogram, $254 \mathrm{~nm}$ ) for the peak corresponding to 4hydroxybenzoic acid at several known concentrations $(0.00 \mu \mathrm{M}, 7.76 \mu \mathrm{M}, 11.64$ $\mu \mathrm{M}, 15.52 \mu \mathrm{M}, 19.40 \mu \mathrm{M}, 23.28 \mu \mathrm{M}, 27.16$ $\mu \mathrm{M}, 31.05 \mu \mathrm{M}, 34.93 \mu \mathrm{M}, 38.81 \mu \mathrm{M})$. The calibration curve is fitted along these points using the least squares method according to the formula $y=a x+b . a$ (slope) and $b$ (intercept at $x=0$ ) are 94533 and -4933 respectively. The measured intensity $y$ for 4-HBA after the $24 \mathrm{~h}$ enzymatic reaction (after 1250x dilution): 1553881. This results in $x_{\text {after_reaction }}=16.24 \mu \mathrm{M}$ from $x_{\text {before_reaction }}$ (diluted sample) $=$ concentration 4 -HBA at time $0=21.89 \mu \mathrm{M}$, which means $26 \% 4-\mathrm{HBA}$ has been converted.

\section{Figure S2. Calibration curve for the determination of the conversion of C3-C3' dimer 1.}

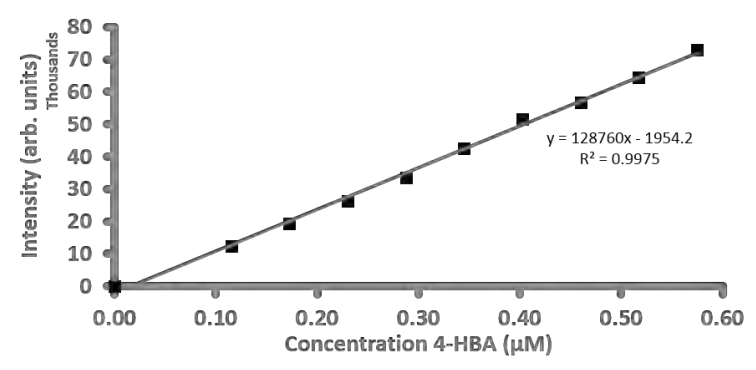

Measured peak areas (UV chromatogram, $254 \mathrm{~nm}$ ) for the peak corresponding to C3-C3' dimer $\mathbf{1}$ at several known concentrations $(0.00 \mu \mathrm{M}, 0.11 \mu \mathrm{M}, 0.17$ $\mu \mathrm{M}, 0.23 \mu \mathrm{M}, 0.29 \mu \mathrm{M}, 0.34 \mu \mathrm{M}, 0.40 \mu \mathrm{M}$, $0.46 \mu \mathrm{M}, 0.52 \mu \mathrm{M}, 0.57 \mu \mathrm{M})$. The calibration curve is fitted along these points using the least squares method according to the formula $y=a x+b$. $a$ (slope) and $b$ (intercept at $x=0$ ) are 128760 and -1954.2 respectively. The measured intensity $y$ for C3-C3' dimer 1 after the $24 \mathrm{~h}$ enzymatic reaction (after 1250x dilution): 26850. This results in $x_{\text {after_reaction }}=0.22 \mu \mathrm{M}$ from $x_{\text {before_reaction }}$ (diluted sample) $=$ concentration 4-HBA at time $0=21.12 \mu \mathrm{M}$, which means $2 \%$ 4-HBA has been converted to C3-C3' dimer $\mathbf{1}$ ( 2 units of 4-HBA convert to 1 unit of dimer).

\section{Figure S3. Calibration curve for the determination of the conversion of C3-O dimer 2.}

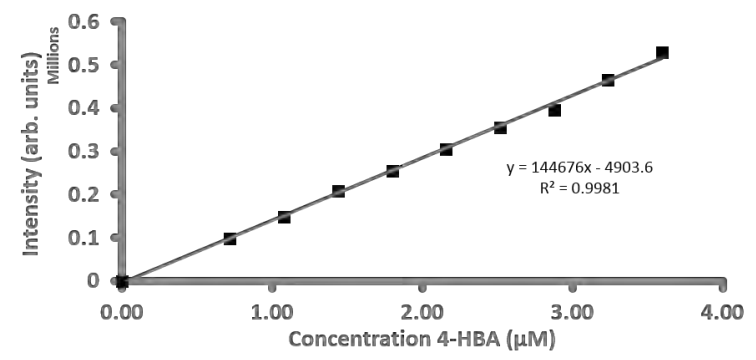

Measured peak areas (UV chromatogram, $254 \mathrm{~nm}$ ) for the peak corresponding to C3-O dimer $\mathbf{2}$ at several known concentrations $(0.00 \mu \mathrm{M}, 0.72 \mu \mathrm{M}, 1.08$ $\mu \mathrm{M}, 1.44 \mu \mathrm{M}, 1.80 \mu \mathrm{M}, 2.16 \mu \mathrm{M}, 2.52 \mu \mathrm{M}$, $2.88 \mu \mathrm{M}, \quad 3.24 \mu \mathrm{M}, 3.60 \mu \mathrm{M})$. The calibration curve is fitted along these points using the least squares method according to the formula $y=a x+b . a$ (slope) and $b$ (intercept at $x=0$ ) are 144676 and -4903.6 respectively. The measured intensity $y$ for C3-O dimer 2 after the $24 \mathrm{~h}$ enzymatic reaction (after 10x dilution): 444224. This results in $x_{\text {after_reaction }}=3.07 \mu \mathrm{M}$ from $x_{\text {before_reaction }}$ (diluted sample) $=$ concentration 4-HBA at time $0=2640 \mu \mathrm{M}$, which means $0.2 \%$ 4-HBA has been converted to C3-O dimer $\mathbf{2}$ ( 2 units of 4-HBA convert to 1 unit of dimer). 
Figure S4. Calibration curve for the determination of the conversion of C1-C3' dimer 3.

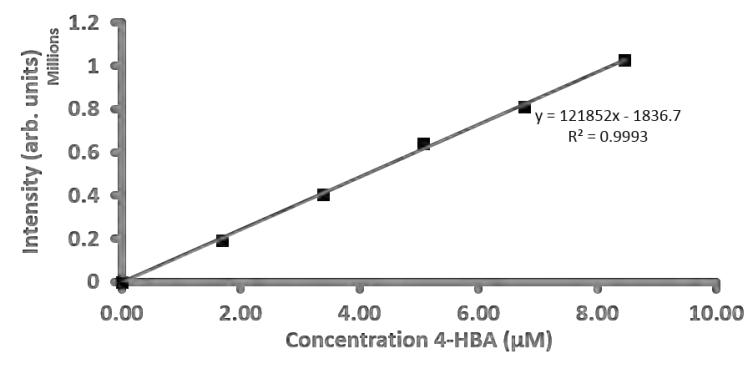

Measured peak areas (UV chromatogram, $254 \mathrm{~nm}$ ) for the peak corresponding to C1-C3' dimer $\mathbf{3}$ at several known concentrations $(0.00 \mu \mathrm{M}, 1.69 \mu \mathrm{M}, 3.38$ $\mu \mathrm{M}, 5.07 \mu \mathrm{M}, 6.77 \mu \mathrm{M}, 8.46 \mu \mathrm{M})$. The calibration curve is fitted along these points using the least squares method according to the formula $y=a x+b$. $a$ (slope) and $b$ (intercept at $x=0$ ) are 121852 and -1836.7 respectively.

Figure S5. Calibration curve for the determination of the conversion of C1-O dimer 4.

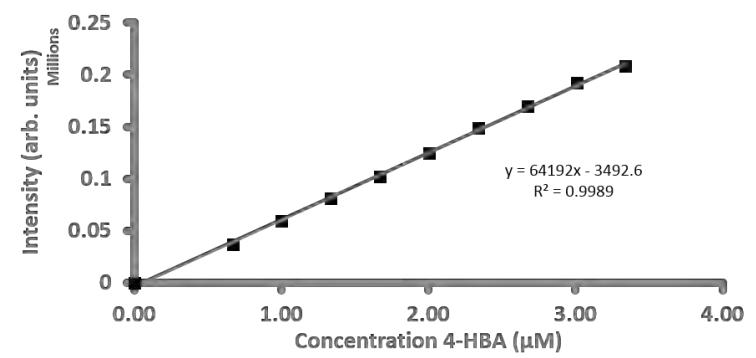

Measured peak areas (UV chromatogram, $254 \mathrm{~nm}$ ) for the peak corresponding to $\mathrm{C} 1-\mathrm{O}$ dimer $\mathbf{4}$ at several known concentrations $(0.00 \mu \mathrm{M}, 0.67 \mu \mathrm{M}, 1.00$ $\mu \mathrm{M}, 1.34 \mu \mathrm{M}, 1.67 \mu \mathrm{M}, 2.00 \mu \mathrm{M}, 2.34 \mu \mathrm{M}$, $2.67 \mu \mathrm{M}, 3.01 \mu \mathrm{M}, 3.34 \mu \mathrm{M})$. The calibration curve is fitted along these points using the least squares method according to the formula $y=a x+b$. $a$ (slope) and $b$ (intercept at $x=0$ ) are 64192 and -3492.6 respectively.

Figure S6. Extracted ion chromatogram for $m / z 137 \pm 0.50 \mathrm{Da}(4-\mathrm{HBA})$.

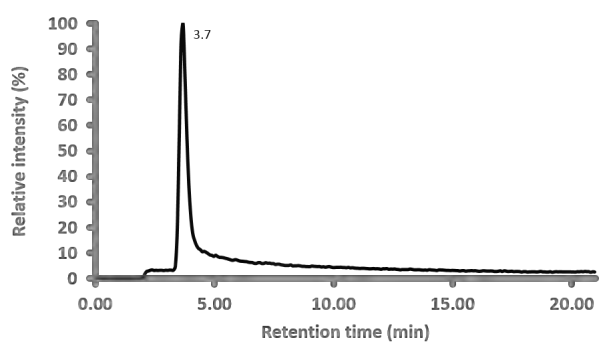

Extracted ion chromatogram for $m / z 137 \pm 0.50$ Da (4HBA) from LC-MS analysis of 4-HBA oligomerisation (LCseparation: LCl, line smoothening applied for clarity).
Figure S7. Mass spectrum corresponding to peak at $\mathbf{3 . 7} \mathrm{min}$ in SI Figure S6.

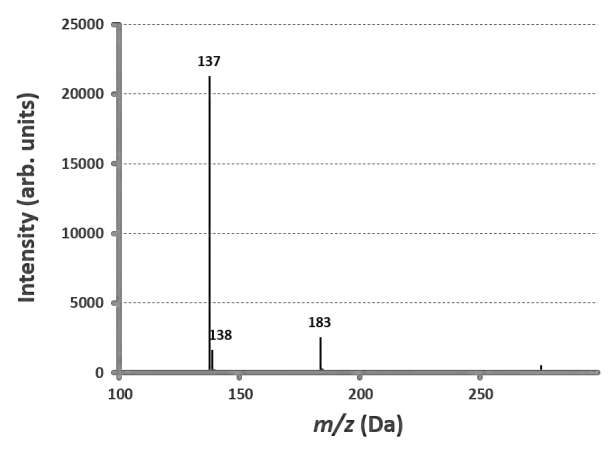


Figure S8. Extracted ion chromatogram for $m / z 273 \pm 0.50 \mathrm{Da}$ (dimers).

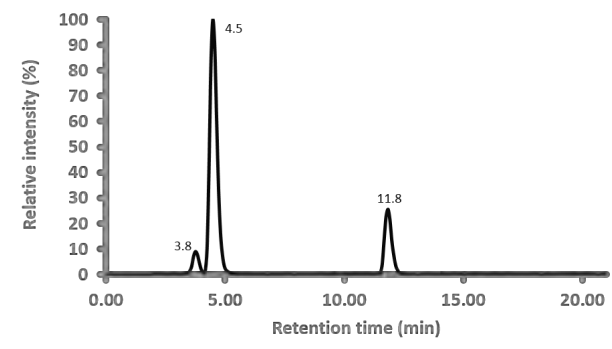

Extracted ion chromatogram for $m / z 273 \pm 0.50 \mathrm{Da}$ (dimers) from LC-MS analysis of 4-HBA oligomerisation (LC-separation: LC1, line smoothening applied for clarity).

Figure S10. Mass spectrum corresponding to peak at $\mathbf{1 1 . 8} \mathbf{~ m i n}$ in SI Figure S8.

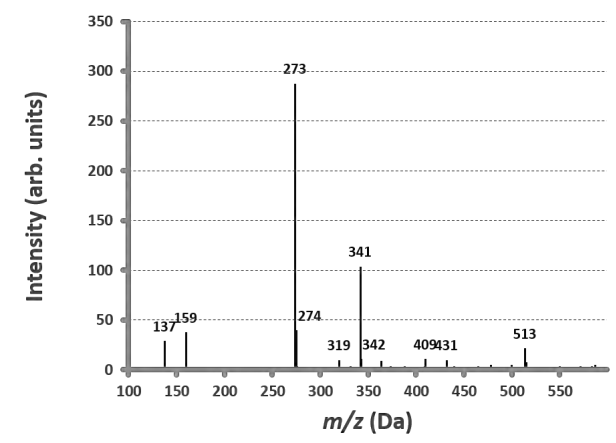

Figure S11. Extracted ion chromatogram for $m / z 379 \pm 0.50 \mathrm{Da}$ (trimeric benzoquinone).

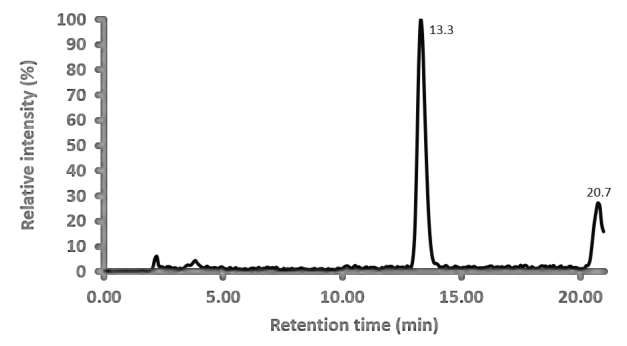

Extracted ion chromatogram for $m / z 379 \pm 0.50$ Da from LC-MS analysis of 4-HBA oligomerisation (LC-separation: $\mathrm{LCl}$, line smoothening applied for clarity).
Figure S9. Mass spectrum corresponding to peak at $4.5 \mathrm{~min}$ in SI Figure S8.

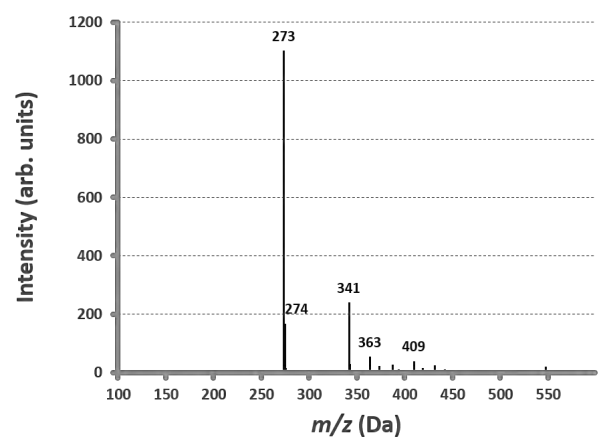

Figure S12. Mass spectrum corresponding to peak at $13.3 \mathrm{~min}$ in SI Figure S11.

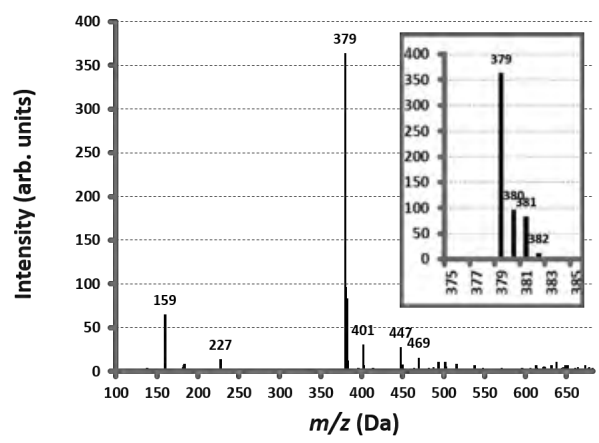


Figure S13. Extracted ion chromatogram for $\mathrm{m} / \mathrm{z} 545 \pm 0.50 \mathrm{Da}$.

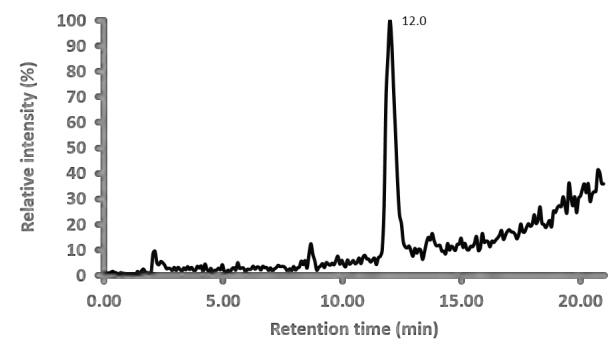

Extracted ion chromatogram for $m / z 545 \pm 0.50$ Da from LC-MS analysis of laccase mediated conversion of 4-HBA and dimer 2 (LC-separation: LC2, line smoothening applied for clarity).

Figure S15. Extracted ion chromatogram for $\mathrm{m} / \mathrm{z} 229 \pm 0.50 \mathrm{Da}$ (dimer 3 and 4).

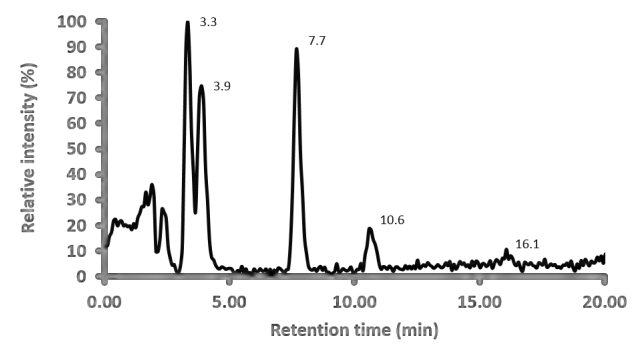

Extracted ion chromatogram for $m / z 229 \pm 0.50 \mathrm{Da}$ (dimer $\mathbf{3}$ and $\mathbf{4}$ ) from LC-MS analysis of laccase mediated conversion of 4-HBA (LC-separation: LC3, line smoothening applied for clarity).
Figure S14. Mass spectrum corresponding to peak at $\mathbf{1 2 . 0} \mathbf{~ m i n}$ in SI Figure S13.

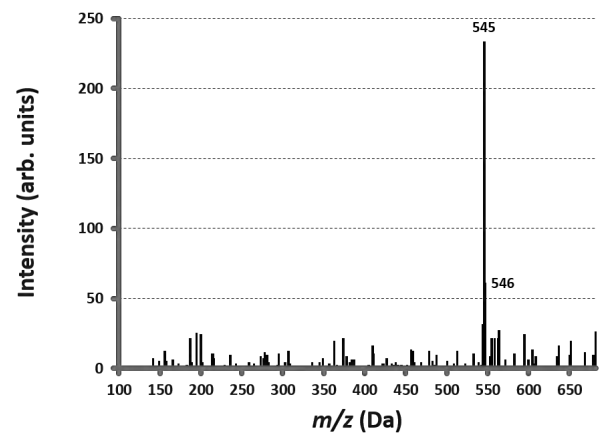

Figure S16. Mass spectrum corresponding to peak at $7.7 \mathrm{~min}$ in SI Figure S15.

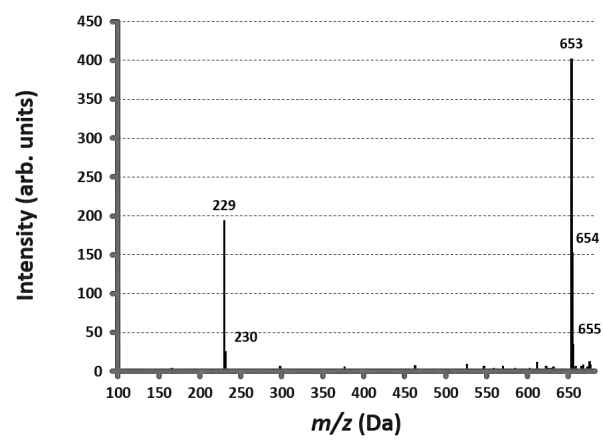


Figure S17. Co-injection dimer 1 and 2 with 4-HBA reaction.

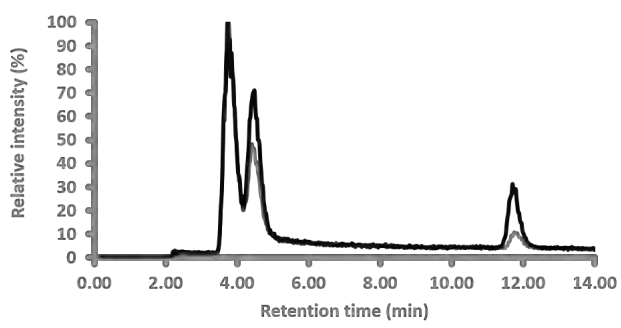

Extracted ion chromatograms $(m / z 137 \pm 0.50 \mathrm{Da}+m / \mathrm{z}$ $273 \pm 0.50 \mathrm{Da})$ of reaction of laccase and 4hydroxybenzoic acid at $24 \mathrm{~h}$ (grey) and the same reaction mixture spiked with chemically synthesised dimer $\mathbf{1}$ and dimer 2 (black). Separation was achieved through LCmethod: LCl.

Figure S19. Co-injection dimer 4 with 4-HBA reaction.

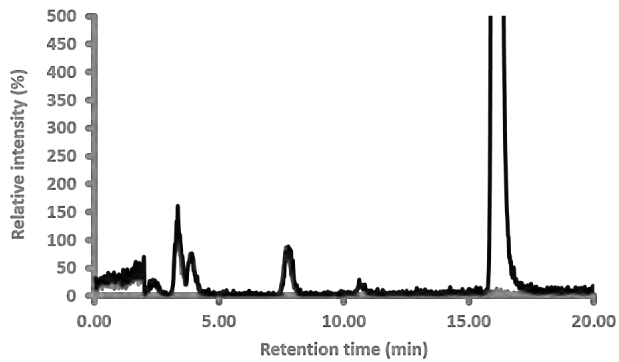

Extracted ion chromatograms $(m / z 229 \pm 0.50 \mathrm{Da})$ of reaction of laccase and 4-hydroxybenzoic acid at $24 \mathrm{~h}$ (grey) and the same reaction mixture spiked with chemically synthesised dimer $\mathbf{4}$ (black). Separation was achieved through LC-method: LC3.

Figure S20. Mass spectrum for chemically synth. dimer 1 at retention time $4.5 \mathrm{~min}$ (LC1).

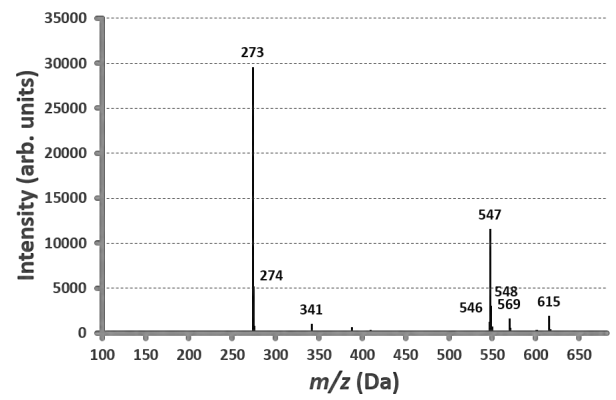

Figure S18. Co-injection dimer 3 with 4-HBA reaction.

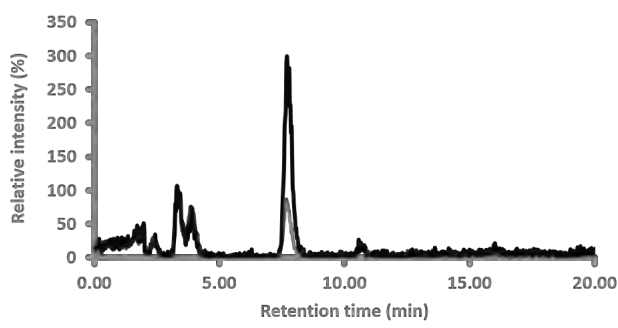

Extracted ion chromatograms $(m / z 229 \pm 0.50 \mathrm{Da})$ of reaction of laccase and 4-hydroxybenzoic acid at $24 \mathrm{~h}$ (grey) and the same reaction mixture spiked with chemically synthesised dimer $\mathbf{3}$ (black). Separation was achieved through LC-method: LC3.
Figure S21. Mass spectrum for chemically synth. dimer 2 at retention time $11.8 \mathrm{~min}$ (LC1).

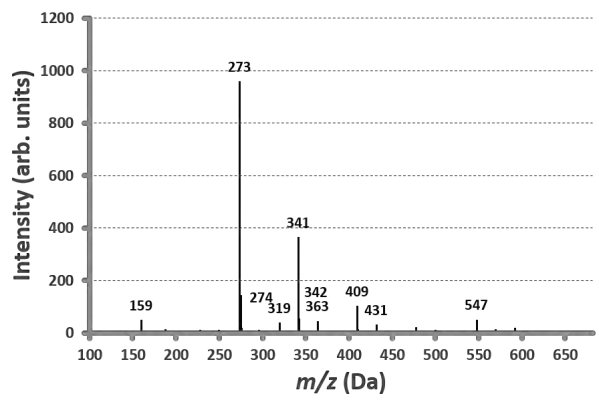


Figure S22. Mass spectrum for chemically synthesised dimer 3 at retention time 7.7 $\min ($ LC3).

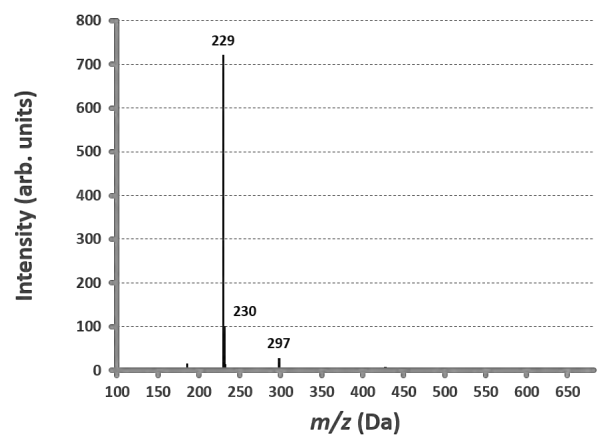

Figure S24. Conversion of dimer 3 by laccase over time.

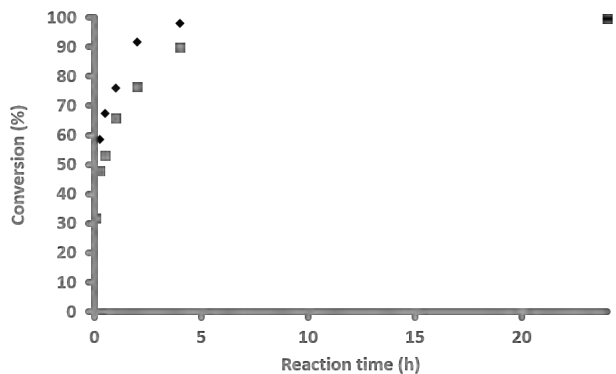

Dimer 3 (1.6 mg, $6.8 \mu \mathrm{mol}, 3.40 \mathrm{mM}$ ) only (black diamonds) or in the presence of 4-hydroxybenzoic acid (grey squares, $0.9 \mathrm{mg}, 6.8 \mu \mathrm{mol}, 3.40 \mathrm{mM}$ ) was reacted in the presence of laccase $\left(0.51 \mathrm{U} \mathrm{ml}^{-1}\right)$ in a mixture of methanol and a $0.02 \mathrm{M}$ ammonium acetate/acetic acid buffer $(1: 4.4)$ at room temperature and $\mathrm{pH}$ 5. The conversion of dimer 3 was monitored at 5 min (not for the reaction with dimer 3 only), $15 \mathrm{~min}, 30 \mathrm{~min}, 1 \mathrm{~h}, 2 \mathrm{~h}, 4 \mathrm{~h}$ and $24 \mathrm{~h}$ by determination of the UV intensity corresponding to the peak of dimer $\mathbf{3}$ after LC-separation and interpolation on the respective calibration curve (SI Figure S4).
Figure S23. Yield of dimer 3 in the laccasemediated oligomerisation of 4-HBA over time.

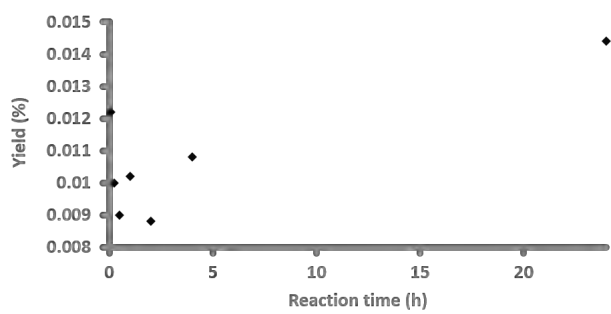

4-HBA (18.5 mg, $0.13 \mathrm{mmol}, 26.8 \mathrm{mM}$ ) and laccase (4.8 $\mathrm{U}, 1.0 \mathrm{U} \mathrm{ml}^{-1}$ ) were reacted in a $0.02 \mathrm{M}$ ammonium

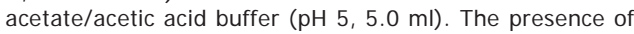
dimer 3 was monitored at $5 \mathrm{~min}, 15 \mathrm{~min}, 30 \mathrm{~min}, 1 \mathrm{~h}, 2$ $\mathrm{h}, 4 \mathrm{~h}$ and $24 \mathrm{~h}$ by determination of UV peak intensity for this dimer after LC-separation and interpolation on the respective calibration curve (SI Figure S4).

Figure S25. Conversion of dimer 4 by laccase over time.

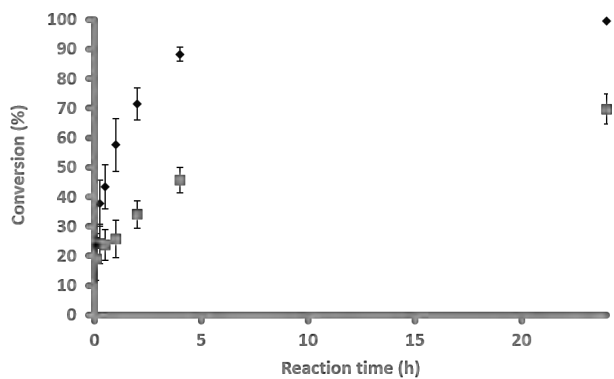

Dimer 4 only (black diamonds, $4.2 \mathrm{mg}, 18.1 \mu \mathrm{mol}, 3.40$ $\mathrm{mM}$ ) or in the presence of 4-hydroxybenzoic acid (grey squares, $2.5 \mathrm{mg}, 18.1 \mu \mathrm{mol}, 3.40 \mathrm{mM}$ ) was reacted in the presence of laccase $\left(0.51 \mathrm{U} \mathrm{ml}^{-1}\right)$ in a mixture of methanol and an ammonium acetate/acetic acid buffer (1:4.4) at room temperature and $\mathrm{pH}$ 5. The conversion of dimer 4 was monitored at $5 \mathrm{~min}, 15 \mathrm{~min}, 30 \mathrm{~min}, 1 \mathrm{~h}, 2 \mathrm{~h}, 4 \mathrm{~h}$ and $24 \mathrm{~h}$ by determination of the UV intensity corresponding to the peak of dimer $\mathbf{4}$ after LC-separation and interpolation on the respective calibration curve (SI Figure S5). The experiment was performed in triplicate except for time point 1 ( $5 \mathrm{~min}$ ) which was conducted in duplicate. Standard deviations over the whole population are included for every time point. 
Figure S26. Optimised geometries for 4-hydroxybenzoic acid and its dimers.
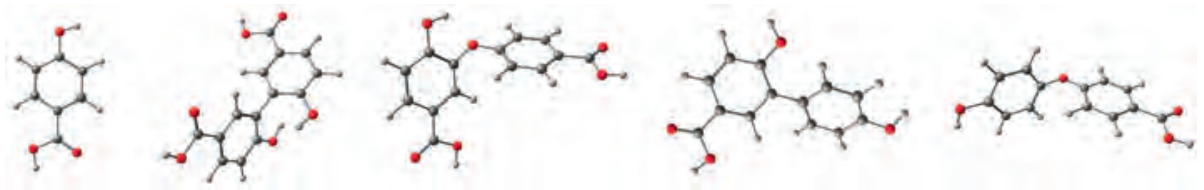

Optimised geometries obtained through quantum chemical calculations at the M11L level $(6-311+G(d, p))$ in water. From left to right: 4-hydroxybenzoic acid, dimer 1, dimer 2, dimer $\mathbf{3}$ and dimer $\mathbf{4}$.

\section{Table S1. Calculated relative energies for the formation of the radical cation.}

Table S1. Calculated relative energies for radical cation formation from 4-HBA and dimers, corresponding to the geometries displayed in SI Figure S26.

\begin{tabular}{cccccc}
\hline Level (solvation) $^{[\mathrm{a}]}$ & \multicolumn{5}{c}{ Energy gap between substrate and its radical cation ${ }^{[\mathrm{b}]}$} \\
& 4-HBA & Dimer 1 & Dimer 2 & Dimer 3 & Dimer 4 \\
\cline { 2 - 6 } M11L (water) & 154.9 & 152.7 & 147.8 & 137.1 & 138.1 \\
M11L (vacuum) & 200.9 & 192.5 & 187.4 & 172.5 & 176.2 \\
B3LYP (vacuum) & 199.1 & 193.2 & 186.6 & 173.8 & 177.6 \\
\hline
\end{tabular}

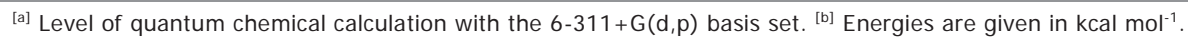

Table S2. Comparison of ${ }^{1} \mathrm{H}$ NMR data for dimers 1 and 2.

Table S2. Comparison of ${ }^{1} \mathrm{H}$ NMR data for both chemically and enzymatically generated dimers $\mathbf{1}$ and $\mathbf{2}$.

\begin{tabular}{|c|c|}
\hline \multicolumn{2}{|c|}{ Dimer $\mathbf{1}^{\text {[a] }}$} \\
\hline Synthetic ( $\delta / \mathrm{ppm})$ & Enzymatic ( $\delta / \mathrm{ppm})$ \\
\hline $6.96\left(\mathrm{~d},{ }^{3} /(\mathrm{H}, \mathrm{H})=9.1 \mathrm{~Hz}, 2 \mathrm{H}\right)$ & $6.94\left(\mathrm{~d},{ }^{3} /(\mathrm{H}, \mathrm{H})=8.4 \mathrm{~Hz}, 2 \mathrm{H}\right)$ \\
\hline $7.94-7.87(\mathrm{~m}, 4 \mathrm{H})$ & $7.95-7.84(\mathrm{~m}, 4 \mathrm{H})$ \\
\hline \multicolumn{2}{|c|}{ Dimer $\mathbf{2}^{[\mathrm{b}]}$} \\
\hline Synthetic ( $\delta /$ ppm) & Enzymatic ( $\delta / \mathrm{ppm})$ \\
\hline $7.02\left(\mathrm{~d},{ }^{3} /(\mathrm{H}, \mathrm{H})=8.8 \mathrm{~Hz}, 2 \mathrm{H}\right)$ & $7.06-6.98(\mathrm{~m}, 2 \mathrm{H})$ \\
\hline $7.16\left(\mathrm{~d},{ }^{3} /(\mathrm{H}, \mathrm{H})=8.5 \mathrm{~Hz}, 1 \mathrm{H}\right)$ & $7.16\left(\mathrm{~d},{ }^{3} /(\mathrm{H}, \mathrm{H})=8.5 \mathrm{~Hz}, 1 \mathrm{H}\right)$ \\
\hline $7.70\left(\mathrm{~d},{ }^{3} /(\mathrm{H}, \mathrm{H})=2.0 \mathrm{~Hz}, 1 \mathrm{H}\right)$ & $7.70\left(\mathrm{~d},{ }^{3} /(\mathrm{H}, \mathrm{H})=2.1 \mathrm{~Hz}, 1 \mathrm{H}\right)$ \\
\hline $7.85\left(\mathrm{dd},{ }^{3} /(\mathrm{H}, \mathrm{H})=8.5,2.0 \mathrm{~Hz}, 1 \mathrm{H}\right)$ & $7.85(\mathrm{dd}, 3 /(\mathrm{H}, \mathrm{H})=8.5,2.1 \mathrm{~Hz}, 1 \mathrm{H})$ \\
\hline $8.03(\mathrm{~d}, 3 /(\mathrm{H}, \mathrm{H})=8.8 \mathrm{~Hz}, 2 \mathrm{H})$ & $8.07-7.98(m, 2 H)$ \\
\hline
\end{tabular}

${ }^{[a]}$ NMR solvent $=$ MeOD. ${ }^{[b]}$ NMR solvent $=$ acetone- $d_{6}$. 


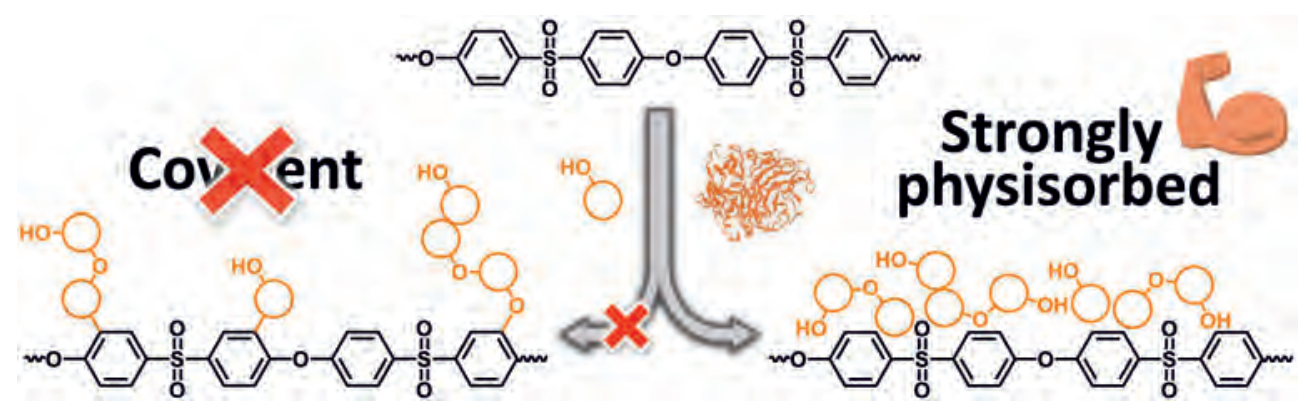




\title{
Chapter 3
}

\section{Elucidating the mechanism behind the laccase-mediated modification of poly(ethersulfone)}

\begin{abstract}
Laccase-mediated oligomerisation of 4-hydroxybenzoic acid (4-HBA) derivatives and simultaneous insitu surface modification has proven to be a cost-effective, easily applicable and eco-friendly strategy for preventing biofouling of poly(ethersulfone) (PES) water filtration membranes. Modification of the membrane surface has previously been hypothesised to occur through covalent bonding of the enzymatically generated phenolic radicals to the polymeric membrane. The current study shows, however, that in-situ formation of soluble phenolic oligomers does not result in covalent membrane modification. We studied in-situ laccase-mediated oligomerisation of custom-synthesised positively charged and commercially available negatively charged monomeric phenols, and demonstrated that their mode of binding to PES is not covalent. In addition, soluble, non-soluble and on-resin PES model compounds were synthesised and used in the laccase-mediated oligomerisation of 4-HBA. Covalent bond formation between these model compounds and (oligomeric) 4-HBA could not be observed either. Furthermore, extensive washing of PES membranes modified through laccase-mediated oligomerisation of 4-HBA resulted in substantial discolouration of the membrane surface, showing that the layer of oligomerised phenolics could easily be removed. Altogether, it was concluded that laccaseassisted modification of PES membranes is the result of strong physical adsorption of oligomers and polymers rather than of covalent bonding.
\end{abstract}

This chapter has been adapted from:

Elucidating the mechanism behind the laccase-mediated modification of poly(ethersulfone) S. Slagman, W. A. Jonkers, H. Zuilhof, M. C. R. Franssen, RSC Adv. 2018, 8, 27101-27110

* This chapter is directly followed by its corresponding supporting information. In order to access NMR spectra, the reader is referred to the online version of the publication mentioned above. 


\section{I ntroduction}

Fouling of synthetic membranes is a major concern in the water purification industry. ${ }^{[1]}$ Fouling results in membrane deterioration and decline of flux (water throughput), which factors are eventually leading to less efficient water purification and higher operating costs. The main cause underlying these issues is biological fouling (biofouling), which is the adherence of biological material, typically initiated by the process of non-specific adsorption of proteins and other biopolymers, followed by biofilm formation. ${ }^{[2]}$ Biofouling can, however, be minimised in various ways: by plasma-assisted coating, ${ }^{[3]}$ chemical grafting ${ }^{[4]}$ or mere blending for example. ${ }^{[5]}$ However, all these methods are either laborious or require harsh, toxic and costly chemicals. ${ }^{[6]}$

As enzymes can be employed at ambient temperature to perform reactions in aqueous media, the use of enzymes to perform complex chemical modifications is continually gaining interest. Apart from their use in the synthesis of small-molecules ${ }^{[7]}$ and polymers, ${ }^{[8]}$ they have also been employed in material surface modification. Within our group we have developed a laccase-based modification strategy to alter the surface of commercially available poly(ethersulfone) membranes (PES membranes). ${ }^{[9]}$ The enzyme laccase generates reactive phenolic radicals from 4-hydroxybenzoic acid (4-HBA) derivatives under ambient conditions; these radicals then undergo dimerisation and subsequent oligomerisation, while concomitantly covering the membrane surface. The resulting overlayer prevents the adsorption of polysaccharides, polyphenols, proteins and microorganisms, while maintaining a high membrane porosity. ${ }^{[10]}$ Since laccase uses oxygen as oxidant and produces water as the only by-product, this is also an environmentally benign process. ${ }^{[11]}$

It has been shown that laccase has a low reactivity towards 4-HBA ( $26 \%$ conversion over $24 \mathrm{~h}$ ) due to the presence of an electron-withdrawing carboxylic acid substituent on the phenol ring. ${ }^{[12]}$ After slow initial dimerisation of 4-HBA, the formed dimers (C3-C3'-, C3-O- and C1-C3'-bound) are rapidly converted to oligomers. The presence of additional electron-donating substituents thus increases phenol reactivity. Additionally, the abundance of these dimers is mainly governed by the rate of subsequent oligomerisation. While these observations only concern solution-phase chemistry, initial NMR studies on dissolved membranes modified with oligomeric 4-HBA revealed the occurrence of an additional set of peaks that differ slightly from the peaks corresponding to non-bound 4-HBA. ${ }^{[9]}$ These new peaks were attributed to 4-HBA that is covalently bound (grafted) to the PES surface. Additional molecular modelling studies further suggested the likelihood of O-bound and C-bound phenolic species being covalently bound to the surface. Altogether, it was deemed highly likely that laccase-mediated functionalisation of PES is the result of covalent binding.

Apart from their use in the water filtration industry, synthetic polymeric membranes (like PES) are often used as support for layer-by-layer (LbL) deposition of polyelectrolytes. These LbL membranes have smaller pore sizes, ${ }^{[13]}$ lower molecular weight cut-off values, ${ }^{[13 b, 13 c]}$ increased chemical resistance, ${ }^{[13 a, 14]}$ are selective towards certain ions [14b, 15] and/or have specific separatory characteristics, ${ }^{[16]}$ which makes them useful in a wide range of (nanofiltration) applications. These support membranes are either non-charged (like PES) or charged (like sulfonated PES). Non-charged supports are often inexpensive, commercially available membranes, but do not bind strongly to the deposited polyelectrolyte. This can be overcome by choosing a charged support membrane; ${ }^{[17]}$ however, these are often less readily available, costly specialty products. A low-cost post-production alternative towards charged support membranes would thus allow for the development of more durable LbL technologies. Additionally, installing permanently positively charged quaternary ammonium moieties is likely to induce anti-bacterial properties to the membrane. ${ }^{[18]}$

We envisioned that laccase's ability to generate highly reactive radicals under mild conditions could also be used to functionalise PES membranes with charged phenolics, analogous to the modification of PES with oligomeric 4-HBA. Here we report on the synthesis of a series of positively charged phenolic monomers and their oligomerisation in the presence of PES membranes or non-porous sheets. The modification process was followed by $\mathrm{X}$-ray photoelectron spectroscopy and static water contact angle 
(SWCA) measurements. The results of these experiments prompted us to additionally study the laccase-mediated conversion of phenolics in the presence of (resin-bound) PES model compounds. All the results taken together shed new light on the nature of the binding modes resulting from laccasemediated modification of PES, which appeared to be different from what was proposed in earlier research.

\section{Results and discussion}

\section{Synthesis of positively charged phenolic monomers}

Laccase-assisted modification of PES membranes with (oligomers of) charged phenolics would grant easy access to anti-bacterial LbL supports. Multiple permanently positively charged phenolics were designed and synthesised for this purpose (Scheme 1). These phenols were expected to possess diverse reactivity patterns based on the presence or absence of substituents on the $\mathrm{C} 3$ and $\mathrm{C} 5$ position of the phenol ring.

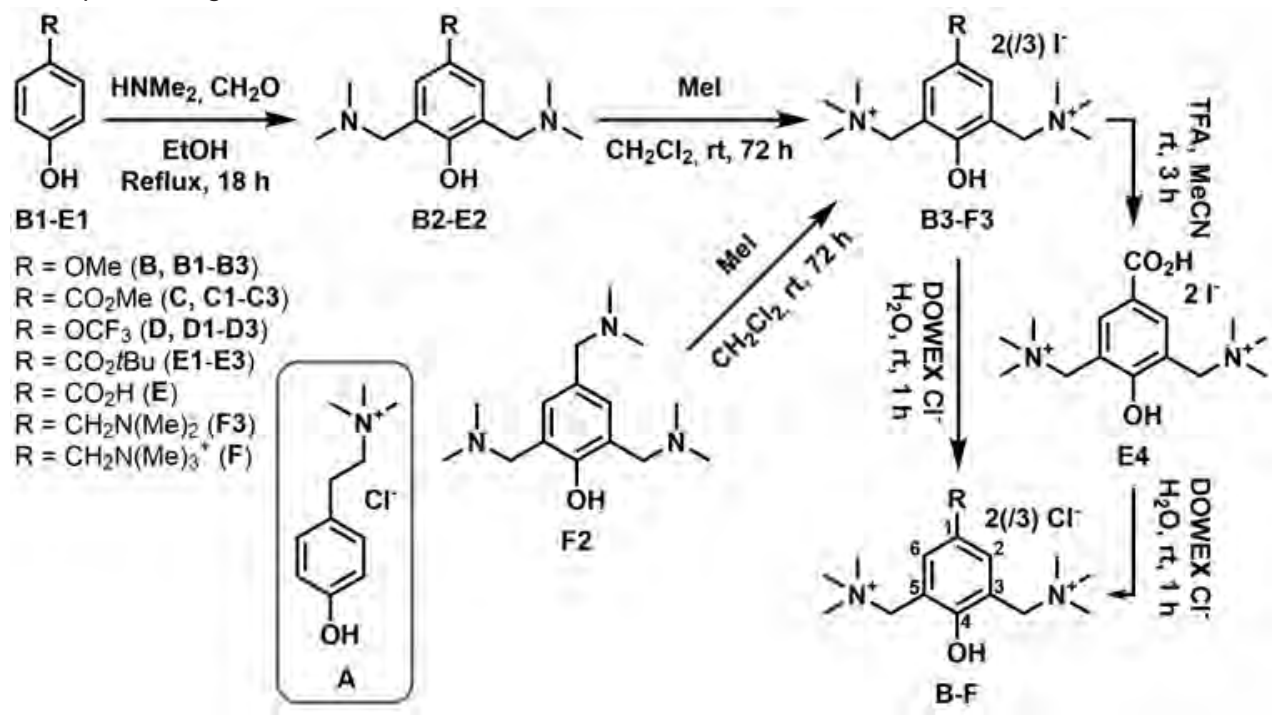

Scheme 1. Synthetic route towards positively charged phenolic monomers B-F.

The simplest positively charged phenol $(N, N, N$-trimethyltyramine chloride (A)) was based on tyramine. Laccase-mediated oligomerisation of $\mathbf{A}$, and its binding to PES, is expected to result in an inhomogeneous mixture of products (Scheme 2), as A is not substituted at its C3 and C5 positions. Tyramine itself is only a poor laccase substrate, ${ }^{[19]}$ it is therefore interesting to see whether quarternising its nitrogen atom will increase reactivity. I nitial attempts towards $\mathbf{A}$, based on the direct methylation of tyramine, resulted in an inseparable mixture (results not shown). ${ }^{[20]}$ Methylation of commercially available $N, N$-dimethylamine followed by ion exchange did, however, afford compound $\mathbf{A}$ in excellent yield.

Laccase-mediated conversion of phenolics B, C and $\mathbf{D}$ is expected to exclusively result in phenolic monomers binding to the surface through $\mathrm{C}-\mathrm{O}$ bond formation with the oxygen-centred radical (Scheme 2). This approach would thereby thus not introduce multilayered oligomers on the surface (as was the case for 4-HBA). B and $\mathbf{D}$ are furthermore likely to be more reactive due to the presence of additional electron-donating substituents. For the synthesis of these doubly charged monomers, parasubstituted phenols (B1-D1) were first converted to their respective Mannich bases. ${ }^{[21]}$ Subsequent methylation of both tertiary nitrogen atoms followed by ion exchange afforded $\mathbf{B}, \mathbf{C}$ and $\mathbf{D}$. OCF $\mathbf{O}_{-}$ 
substituted compound $\mathbf{D}$ is of special interest as the presence of multiple fluorine atoms would allow for easy detection of surface-bound $\mathbf{D}$ through X-ray photoelectron spectroscopy (XPS).

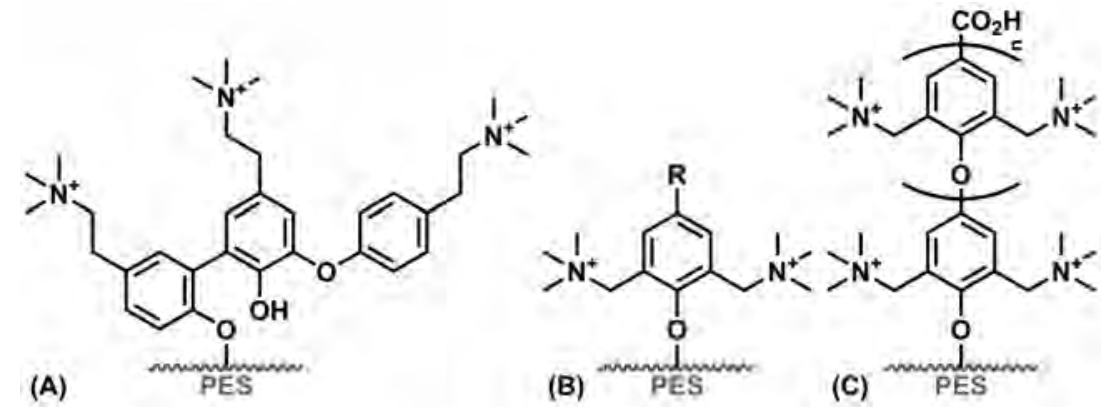

Scheme 2. Anticipated modes of laccase-mediated surface functionalisation, A: with phenolic monomer A, B: with phenolic monomers B-D or $\mathbf{F}$ and C: with phenolic monomer $\mathbf{E}$.

Syringic acid was shown to be effectively polymerised ( $M W$ up to $18 \mathrm{kDa}$ ) by laccase through $\mathrm{Cl}-\mathrm{O}$ bond formation. ${ }^{[22]}$ It was anticipated that laccase-mediated conversion of E would, analogously, result in polymer formation, and that $\mathbf{E}$ could thus, concomitantly, be used to decorate PES membranes with a multilayer of these highly ordered polymers (Scheme 2). Initial attempts to obtain $\mathbf{E}$ in a manner analogous to the synthesis of B-D through Mannich base formation of 4-HBA resulted in a mixture of meta- and ortho-substituted phenol derivatives (results not shown). None of the components of this mixture proved to be compatible with the methylation conditions employed thereafter. Mannich base formation from methyl 4-hydroxybenzoate followed by methylation and deprotection using hydrochloric acid resulted in decarboxylation (results not shown). Finally, E could be obtained by starting from tert-butyl 4-hydroxybenzoate (E1) and deprotection of the tert-butyl ester with trifluoroacetic acid.

The use of triply charged derivative $\mathbf{F}$ is expected to locally introduce a very high charge density on the PES membrane. F was obtained through methylation of commercially available 2,4,6tris(dimethylaminomethyl)phenol (F2), followed by ion exchange. Ion exchange was performed for all phenolics A-F in order to replace the easily oxidisable iodide ion, as we envisioned that its laccasemediated oxidation would interfere with oxidation of A-F. ${ }^{[23]}$ Intermediate $\mathbf{F 3}$ could be purified substantially through precipitation from methanol, the success of which was demonstrated using XPSanalysis (SI Figure S15). XPS N 1s narrow scans of the purified compound showed that conversion towards the triply charged species was nearly quantitative, as almost no neutral (tertiary) nitrogen (400.4 eV) could be observed.

In summary, six phenols (A-F) with distinct charge densities were synthesised. The structural variety should allow for rigorous testing of the mechanism of the laccase-mediated oligomerisation: A is likely resulting in an inhomogeneous mixture of products due to the free $\mathrm{C} 3$ and $\mathrm{C} 5$ positions, where $\mathbf{B}, \mathbf{C}, \mathbf{D}$ and $\mathbf{F}$ can only bind to the surface through C-O bond formation, while the use of $\mathbf{E}$ is expected to result in polymers in which its monomeric units will be consistently $\mathrm{Cl}$-O-bound (Scheme 2).

\section{Laccase-mediated conversion of charged monomers in the presence of PES membranes}

Initial attempts at functionalising PES membranes using laccase and positively charged $N, N, N-$ trimethyltyramine chloride (A) resulted in a coloured (faintly yellow) reaction medium. Colouration could indicate extension of a $\pi$ system, which thus hinted at oligomerisation through C-C bond formation. NMR analysis confirmed successful conversion of the starting material to a plurality of products (Figure 1). 


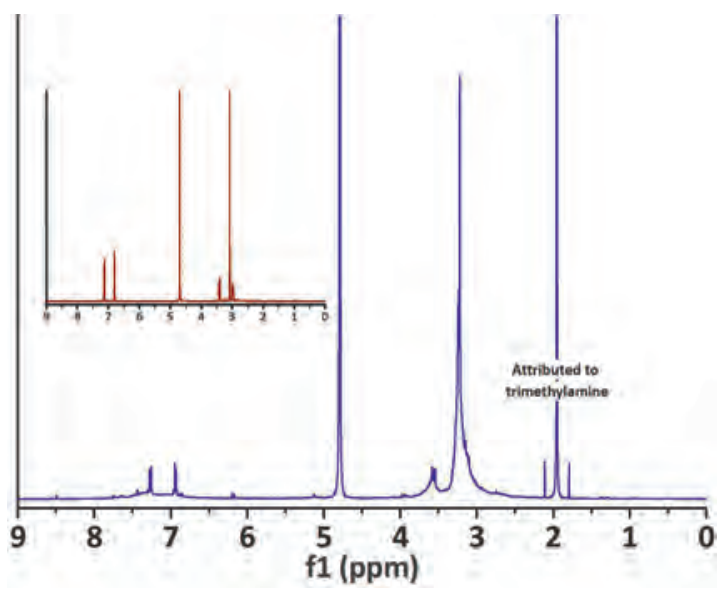

Figure 1. ${ }^{1} \mathrm{H}$ NMR spectrum of the reaction medium containing laccase $\left(1 \mathrm{U} \mathrm{ml}^{-1}\right)$ and $\mathbf{A}$ after $24 \mathrm{~h}$, showing conversion of $\mathbf{A}$ and the appearance of a new singlet at $1.95 \mathrm{ppm}$. Inset: ${ }^{1} \mathrm{H}$ NMR spectrum of $\mathbf{A}$.

Oligomerisation of A did, however, not result in membrane colouration as was observed for the laccase-mediated oligomerisation of 4-HBA. ${ }^{\left[{ }^{[9}\right.}$ Furthermore, XPS analysis of the membrane surface did not reveal the presence of a peak corresponding to quaternary (positively charged) nitrogen at $\sim 502$ eV (Table 1, entry 1, Figure 2). In an attempt to enhance conversion and subsequent coupling of $\mathbf{A}$ to the surface, laccase concentrations were quadrupled. As expected, this resulted in stronger colouration of the reaction medium. Formation of a precipitate further indicated conversion towards (less soluble) higher-order oligomers. Evolution of a strong amine-like odour, combined with the observation of an intense singlet at $1.95 \mathrm{ppm}$ in NMR, however, indicated the formation of trimethylamine (Figure 1). It was hypothesised that formation of trimethylamine resulted from deamination of (oligomers of) A with concomitant formation of 4-vinylphenol (derivatives). Although oligomerisation did not result in membrane colouration, XPS analysis revealed the presence of a peak at $401.6 \mathrm{eV}$ corresponding to quaternary nitrogen $\left(\mathrm{N}^{+}\right.$, Table 1 , entry 2 , Figure 2$)$. Thus, even though deamination seems to have occurred, successful surface functionalisation had been achieved.
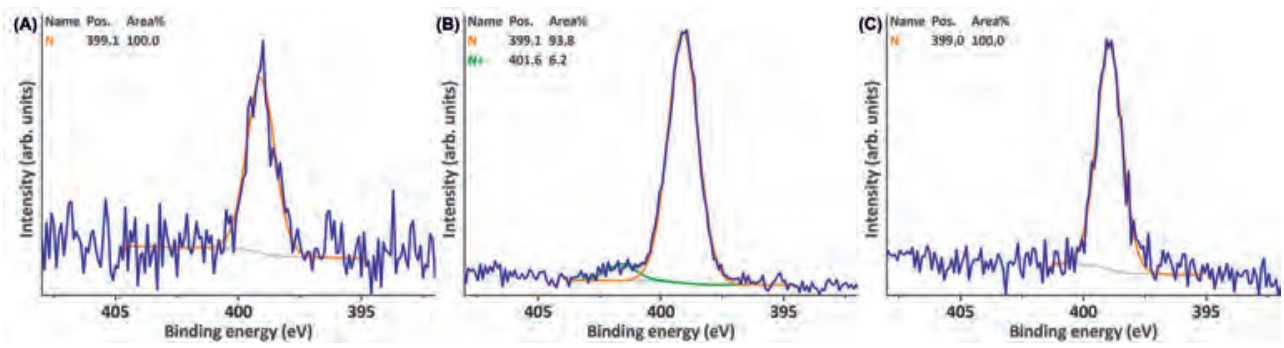

Figure 2. XPS N 1s narrow scans of PES membranes modified through oligomerisation of $\mathbf{A}$ at $A$ : a laccase concentration of $1 \mathrm{U} \mathrm{ml}^{-1}$ and $\mathrm{B}$ : a laccase concentration of $4 \mathrm{U} \mathrm{ml}^{-1}$. C: XPS N is narrow scan of a blank PES membrane.

The use of (PES) membranes adds additional challenges to the analysis of their surface properties. Firstly, since membranes are porous they do not allow for static water contact angle (SWCA) measurements. This assessment of surface wettability would be highly valuable as surface modification with charged phenolics was anticipated to result in a significantly lower SWCA. Secondly, PES membranes contain $10-20 \%$ poly(vinylpyrrolidone) as pore former. ${ }^{[24]}$ The presence of this nitrogencontaining polymer (Table 1, entry 18, Figure 2) hampered further analysis, because functionalisation with the charged phenolics would thus not introduce a unique element. In order to ease analysis, the experiments described below have been conducted on PES sheets rather than on membranes. 
Conversion of doubly charged phenols $\mathbf{B}$ and $\mathbf{C}$ at high laccase concentrations ( $4 \mathrm{U} \mathrm{ml}^{-1}$ ) resulted in a deeply red and deeply yellow reaction medium, respectively, without concomitant formation of odours. Where the use of $\mathbf{B}$ did lead to the presence of a peak corresponding to quaternary nitrogen $(401.6 \mathrm{eV}$ ) in the XPS N 1s narrow scan (Table 1, entry 3, SI Figure S2), the use of $\mathbf{C}$ did not (Table 1, entry 8, SI Figure S6). This difference is likely to be caused by the fact that $\mathbf{B}$ is more reactive, as it bears a stronger electron-donating (methoxy) substituent than $\mathbf{C}$ does (methyl ester). Further increasing the concentration of $\mathbf{B}$ (from $28.8 \mathrm{mM}$ to $57.6 \mathrm{mM}$ ) and increasing the reaction temperature to $40{ }^{\circ} \mathrm{C}$ resulted in a more intense $\mathrm{N}^{+}$signal (Table 1 , entry 6 , Figure 3 ). Increasing the reaction temperature to $40^{\circ} \mathrm{C}$ did not lead to the presence of quaternary nitrogen on the surface when using $\mathbf{C}$ (Table 1, entry 9, SI Figure S7). For the use of both B and C, the presence of a strong (neutral) nitrogen signal ( $399 \mathrm{eV}$ ) was observed (Table 1, entry 3, 6, 8 and 9, Figure 3). As the used PES sheets did not contain any form of nitrogen (Table 1, entry 19, Figure 3), this must have resulted from laccase-assisted surface modification. It was hypothesised that the observed nitrogen originates from unintentional enzyme immobilisation, in other words: protein fouling.
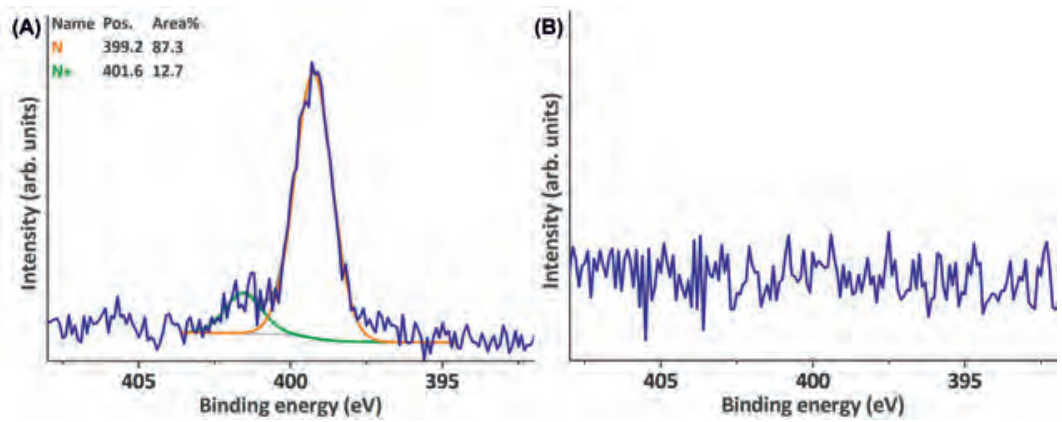

Figure 3. XPS $N$ 1s narrow scans of A: a PES sheet modified through laccase-mediated oligomerisation of B (57.6 $\mathrm{mM}, 40^{\circ} \mathrm{C}$ ) and $\mathrm{B}$ : a blank PES sheet.

As with the use of $\mathbf{C}$, the use of $\mathrm{OCF}_{3}$-substituted $\mathbf{D}$ did not result in successful surface functionalisation; neither fluorine ( $688 \mathrm{eV})$, nor quaternary nitrogen were present on the surface after modification (Table 1, entry 12, SI Figure S8 and S9). Similar to all previous experiments, the use of D resulted in the presence of surface-bound neutral nitrogen-containing species. As a control experiment, D was also used without providing laccase to the reaction mixture (Table 1, entry 13 , Figure 4), which, obviously, did not lead to surface functionalisation with the phenolic monomer. Additionally, no nitrogen was observed on PES sheets used in these control experiments. Furthermore, experiments in which PES sheets were merely agitated in buffer in which laccase was dissolved did result in the observation of surface-bound nitrogen (399.4 eV, Table 1, entry 16, Figure 4).
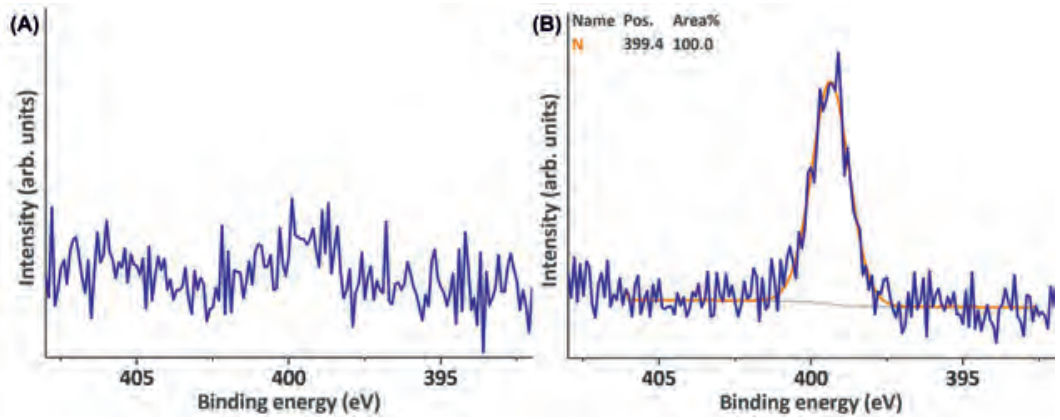

Figure 4. XPS N 1s narrow scans of A: a PES sheet after incubation with D (without addition of laccase) and B: a PES sheet after incubation with laccase. 
As the commercial laccase enzyme is highly impure, it was purified in order to unambiguously determine the cause of these nitrogen-containing contaminations. Purification (SI Figure S34) of the brown laccase powder resulted in a clear blue solution due to the presence of copper (II) ions in the enzyme active site. However, also experiments in which the laccase-mediated conversion of $\mathbf{A}$ was performed using purified laccase led to the presence of additional nitrogen-containing species on the membrane (SI Figure S12 and S13). It can thus be concluded that laccase-mediated conversion of phenolics has also led to immobilisation of the enzyme itself (protein fouling).

Laccase-mediated surface functionalisation using triply charged $\mathbf{F}$ is expected to introduce a high charge density per immobilised molecule. $\mathbf{F}$ is hypothesised to be less reactive than $\mathbf{B}$ due to the poor electron-donating capacity of the $\mathrm{CH}_{2} \mathrm{~N}_{(}\left(\mathrm{CH}_{3}\right)_{3}{ }^{+}$substituent. Nonetheless, a (minor) $\mathrm{N}^{+}$peak was observed after laccase-mediated modification of PES sheets (Table 1, entry 14, SI Figure S10).

To summarise, successful laccase-mediated surface functionalisation was achieved for $\mathbf{A}$, B and $\mathbf{F}$. For the use of all three, however, the peak corresponding to $\mathrm{N}^{+}(\sim 402 \mathrm{eV})$ was observed to be relatively small. As was shown for pure $\mathbf{F}$, the presence of quaternary nitrogen atoms on the surface would have resulted in an intense XPS signal (SI Figure S15). It was therefore concluded that surface coverage after laccase-mediated oligomerisation was minimal in all cases.

Table 1. Presence of neutral and/or positively charged nitrogen-containing species and static water contact angles on (modified) poly(ethersulfone) surfaces.

\begin{tabular}{|c|c|c|c|c|c|c|c|}
\hline \# & Phenol & [phe] ${ }^{[a]}$ & {$[\mathrm{lac}]^{[b]}$} & $\mathbf{T}^{[c]}$ & SWCA ${ }^{[d]}$ & $\mathbf{N}^{+}[\mathbf{e}]$ & $\mathbf{N}^{[\mathrm{f}]}$ \\
\hline 1 & A & 28.8 & 1 & rt & n.d. ${ }^{[g]}$ & No & Major \\
\hline 2 & A & 28.8 & 4 & rt & n.d. & Minor & Major \\
\hline 3 & B & 28.8 & 4 & rt & $51^{\circ}$ & Minor & Major \\
\hline 4 & B & 57.6 & 4 & rt & $60^{\circ}$ & n.d. & n.d. \\
\hline 5 & B & 28.8 & 4 & $40^{\circ} \mathrm{C}$ & $57^{\circ}$ & n.d. & n.d. \\
\hline 6 & B & 57.6 & 4 & $40^{\circ} \mathrm{C}$ & $60^{\circ}$ & Major & Major \\
\hline 7 & $B^{[h]}$ & 57.6 & 4 & $40^{\circ} \mathrm{C}$ & $72^{\circ}$ & No & Minor \\
\hline 8 & $C$ & 28.8 & 4 & rt & $59^{\circ}$ & No & Major \\
\hline 9 & C & 57.6 & 4 & rt & $57^{\circ}$ & No & Major \\
\hline 10 & C & 28.8 & 4 & $40^{\circ} \mathrm{C}$ & $61^{\circ}$ & n.d. & n.d. \\
\hline 11 & C & 57.6 & 4 & $40^{\circ} \mathrm{C}$ & $56^{\circ}$ & n.d. & n.d. \\
\hline 12 & $\mathrm{D}$ & 28.8 & 4 & rt & $58^{\circ}$ & No & Major \\
\hline 13 & D & 28.8 & 0 & rt & $77^{\circ}$ & No & No \\
\hline 14 & $\mathrm{~F}$ & 28.8 & 4 & rt & $55^{\circ}$ & Minor & Major \\
\hline 15 & $F^{[h]}$ & 28.8 & 4 & rt & $78^{\circ}$ & n.d. & n.d. \\
\hline 16 & None & 0 & 4 & rt & $56^{\circ}$ & No & Major \\
\hline 17 & None & 0 & 4 & $40^{\circ} \mathrm{C}$ & $58^{\circ}$ & n.d. & n.d. \\
\hline $18^{[\mathrm{i}]}$ & None & 0 & 0 & - & n.d. & No & Major \\
\hline $19^{[j]}$ & None & 0 & 0 & - & $74^{\circ}$ & No & No \\
\hline
\end{tabular}

${ }^{\text {[a] }}$ Concentration of respective phenol in the reaction mixture $(\mathrm{mM}) .{ }^{[\mathrm{b}]}$ Concentration of laccase in the reaction mixture $\left(\mathrm{U} \mathrm{ml}{ }^{-1}\right) .{ }^{[c]}$ Reaction temperature. ${ }^{[d]}$ Static water contact angle of a $3 \mu \mathrm{l}$ water droplet on the (modified) surface. ${ }^{[\mathrm{e}]}$ Presence of positively charged quaternary nitrogen based on the XPS N is narrow scan. [f] Presence of neutral nitrogen based on the XPS N 1s narrow scan. ${ }^{\left[{ }^{[g]}\right.}$ n.d. = not determined. ${ }^{[\mathrm{h}]}$ Surfaces were wiped after modification. ${ }^{[\mathrm{i}]}$ Blank PES membrane. ${ }^{[j]}$ Blank PES sheet.

The use of PES sheets rather than membranes allowed for SWCA measurements to be conducted. Due to the strongly hydrophilic nature of charged molecules, the SWCA was anticipated to drop significantly after surface functionalisation. Whichever charged phenol was employed, the SWCA dropped from $74^{\circ}$ (Table 1, entry 19) to approximately 50-60 (Table 1, entry 3-6, 8-12, and 14). This was, however, also observed in cases where no $\mathrm{N}^{+}$was observed after surface modification, and also in a control experiment without phenolics in the reaction medium (Table 1, entry 8, 9, 12, 16 and 17). Experiments in which laccase was not added to the reaction mixture (Table 1, entry 13) did not result in a drop in SWCA. It was therefore concluded that unintentional adsorption of the laccase enzyme caused the observed change in wettability. To remove the adsorbed enzyme, the surface of the modified PES sheets was gently wiped with a soft cotton swab. This indeed resulted in a significant 
lowering of the amount of nitrogen on the surface (Table 1, entry 7, SI Figure S4 and S5). Correspondingly, an increase in SWCA was observed, back to the level of unmodified PES. However, as shown through $\mathrm{N}$ 1s XPS spectra, wiping also resulted in the complete removal of any species containing quaternary nitrogen.

Gentle wiping was not expected to result in the removal of covalently bound small organics such as the phenolics used in this study. The removal of nitrogen-containing species would thereby thus indicate that surface modification is not covalent in these cases, which is in contrast with previous research that indicated that laccase-mediated surface modification of PES using 4-hydroxybenzoic acid derivatives is the result of covalent bonding. ${ }^{[9]}$ As the results stated above suggest that surface functionalisation using charged phenolics occurs through an alternative mechanism, it was decided to conduct more research towards the nature of the linkage between PES and 4-HBA (derivatives). By doing so we hope to clarify why 4-HBA adheres to the surface covalently and charged phenolics do not.

\section{Laccase-mediated oligomerisation of 4-hydroxybenzoic acid derivatives in the presence of PES model compounds}

Laccase-mediated surface functionalisation of PES membranes using 4-hydroxybenzoic acid derivatives has proven to be a mild, eco-friendly, easily applicable and cost-effective way of inducing anti-fouling properties to these membranes. ${ }^{[10 \mathrm{a}]}$ It is assumed that this is the result of covalent surface functionalisation. It is, however, difficult to directly observe the bond between the membrane and phenolic compounds, as I) this bond is very similar to bonds between two 4-HBA units, and II) the bulk will still consist mainly of unmodified PES. Probing the modes of binding between PES and (oligomeric) 4-HBA required us to first study comprehensible molecules (model compounds) that have been subjected to the same conditions. Subsequent experiments in which laccase-mediated surface modification has been performed using negatively charged phenols, act as positive controls. Additionally, washing the membranes that have undergone laccase-mediated modification using 4-HBA should subsequently give insight into the degree of grafting.

Studying how laccase-mediated oligomerisation alters lignin model compounds has proven to be an indispensable tool for studying grafting on lignin. ${ }^{[25]}$ Lignin model compounds are (soluble) ligninlike monomers or oligomers that bear some of the functional groups present in lignin. These model compounds are small enough to be separated and/or studied by LC-MS and NMR spectroscopy. Analogously, we aimed to study laccase-mediated functionalisation of (soluble) PES model compounds to mimic laccase-mediated grafting on PES.

For this purpose, two PES model compounds were synthesised; water-insoluble 4,4'sulfonylbis(methoxybenzene) (G, Scheme 3) and water-soluble 2,2'-((sulfonylbis $(4,1-$ phenylene))bis(oxy))-diacetic acid (H). G was afforded in one step through methylation of 4,4'sulfonyldiphenol. Etherification of both alcohol moieties of 4,4'-sulfonyldiphenol using ethyl chloroacetate followed by basic hydrolysis of the methyl esters afforded $\mathbf{H}$ in good yield.

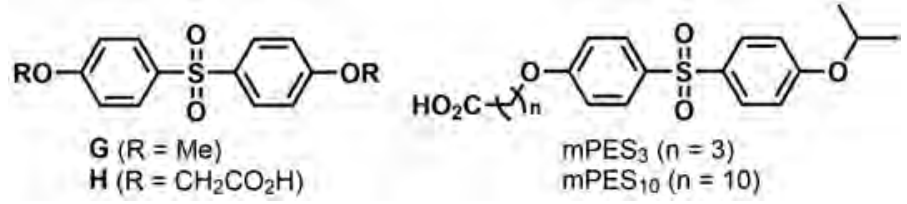

Scheme 3. PES model compounds used in this study.

4-HBA, laccase and either of the PES model compounds $\mathbf{G}$ or $\mathbf{H}$ were agitated at $\mathrm{pH}$ 5, followed by LC-MS analysis. Successful grafting on these PES model compounds would be indicated by the formation of "PES model compound/(oligomeric) 4-HBA conjugates". Laccase-mediated oligomerisation of 4-HBA has been shown to firstly result in C-C- or C-O-bound dimers with $m / z 273.04$ (diml, Scheme 
4) as most abundant products and also in dimers with $m / z 229.05$ (dim2). ${ }^{[12]}$ Our initial targeted search therefore focussed on conjugates of either of the PES model compounds with 4-HBA or its dimers. As an example: a conjugate between model compound $\mathbf{G}$ and a dimer with $m / z 273.04$ will be denoted as G/diml (calculated $m / z$ 549.09).

(A)

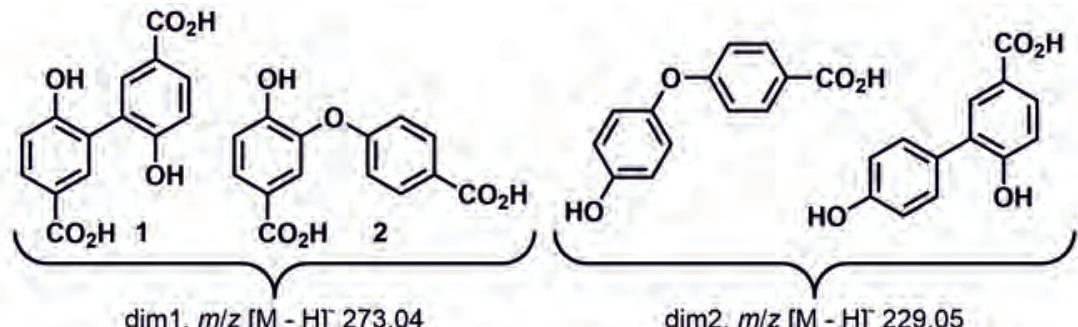

(B)

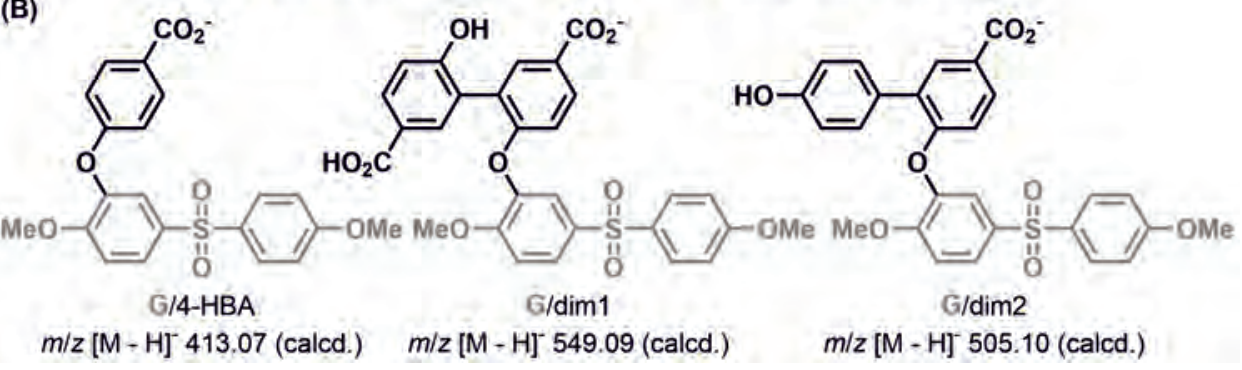

Scheme 4. A: Dimers formed during solution-phase laccase-mediated oligomerisation of 4-HBA. B: Structures of possible conjugates formed after laccase-assisted conversion of 4-HBA in the presence of PES model compound G (each of the depicted conjugates is merely an example of one of many possible isomers).

Attachment of 4-HBA or any of its dimers to $\mathbf{G}$ could not be observed (Figure 5). The use of $\mathbf{H}$ was expected to result in increased conjugate formation due to better mass transfer, as $\mathbf{H}$ is soluble in water. However, coupling of 4-HBA or its dimers to $\mathbf{H}$ could not be detected either (SI Figure S16). Products that could be observed were merely the result of laccase-mediated oligomerisation of 4-HBA. Attempts at increasing the conversion of 4-HBA by adding a mediator (TEMPO) did not result in H/dim1, H/dim2 or H/4-HBA conjugates either (SI Figure S17). Performing LC-MS analysis at higher concentrations allowed for easier (semi-)untargeted detection of conjugates. In this way the mass spectra at retention times corresponding to significant peaks in the UV chromatogram were analysed for plausible conjugates in which the bound (oligomeric) 4-HBA fragment would have undergone additional transformations e.g. further oligomerisation, hydroxylation or oxidation (SI Figure S18-S26). However, none of the observed peaks corresponded to a possible conjugate between $\mathbf{H}$ and 4-HBA derivatives/oligomers.
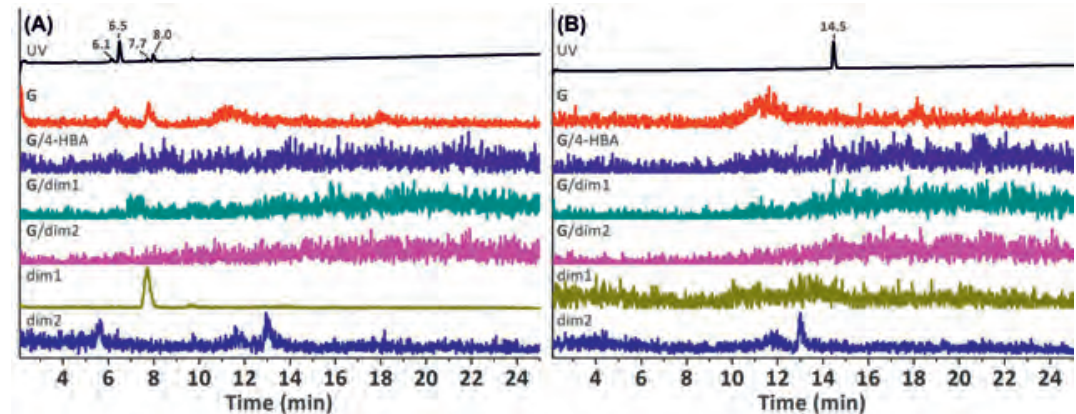

Figure 5. UV trace and extracted ion chromatograms of ions whose $m / z\left(\left[\mathrm{M}-\mathrm{H}^{-} \pm 0.50 \mathrm{Da}\right)\right.$ corresponds to that of G, $\operatorname{dim} 1 / \operatorname{dim} 2$ or their conjugates after incubation of 4-HBA, laccase and G. A: Aqueous reaction medium and B: ethyl acetate extract of solids. 
In a final attempt to induce conjugation, a more reactive phenol (vanillic acid) was subjected to laccase-mediated oligomerisation in the presence of PES model compound $\mathbf{H}$. Analogous to the laccase-mediated dimerisation of 4-HBA, vanillic acid (VA) is known to form C3-C3'-bound dimers (VAdim1, calculated $m / z$ 333.06).[26] Two other abundantly present dimers were the result of monomer coupling and decarboxylative hydroxylation followed by oxidation (H/VAdim2, calculated $\mathrm{m} / \mathrm{z}$ 303.05). However, none of the expected conjugates H/VA, H/VAdim1 or H/VAdim2 were observed (SI Figure S27). Similar to the laccase-mediated oligomerisation of 4-HBA in the presence of $\mathbf{H}$, the products that were observed, were vanillic acid oligomers.

It appeared that neither 4-HBA nor VA were reactive towards the model compounds $\mathbf{H}$ or $\mathbf{G}$. Either the proposed conjugates were not formed and untargeted search was not effective, or the quantities of these conjugates were too low to observe against the background of other molecules. To test whether the latter statement is correct we aimed to separate bulk non-conjugate molecules from the formed conjugates.

\section{Laccase-mediated oligomerisation of 4-hydroxybenzoic acid derivatives in the presence of resin-bound PES model compounds}

Laccase-mediated oligomerisation in the presence of (soluble) PES model compounds did not lead to the observation of PES model compound/(oligomeric) 4-HBA conjugates. To ease analysis, separation between the conjugates and non-conjugates (starting material and oligomeric 4-HBA) would be desirable. Due to a high similarity between both, and the low abundance of conjugates, chromatographic separation after laccase-mediated oligomerisation would be nearly impossible. We therefore envisioned immobilising a PES model compound on a resin in order for it to be isolable from the rest of the reaction medium through simple washing and filtering. This is accompanied with the additional advantage that the resin-bound PES model compound (R-mPES) would more accurately resemble solid PES fibres that are restricted in their mobility. As depicted in Scheme 5, the monohydroxy-functionalised PES model compound was first functionalised with a carboxylic acid to enable coupling to the resin to afford the resin-bound model compound; R-mPES. Laccase-mediated oligomerisation in the presence of R-mPES took place subsequently, which was followed by extensive washing of R-mPES to remove all non-bound starting material and oligomers of 4-HBA. Cleavage of the model compound and its reaction products from the resin, followed by LC-MS analysis allowed for the detection of possible conjugates and was expected to thereby reveal the nature of the bond between PES and 4-HBA.

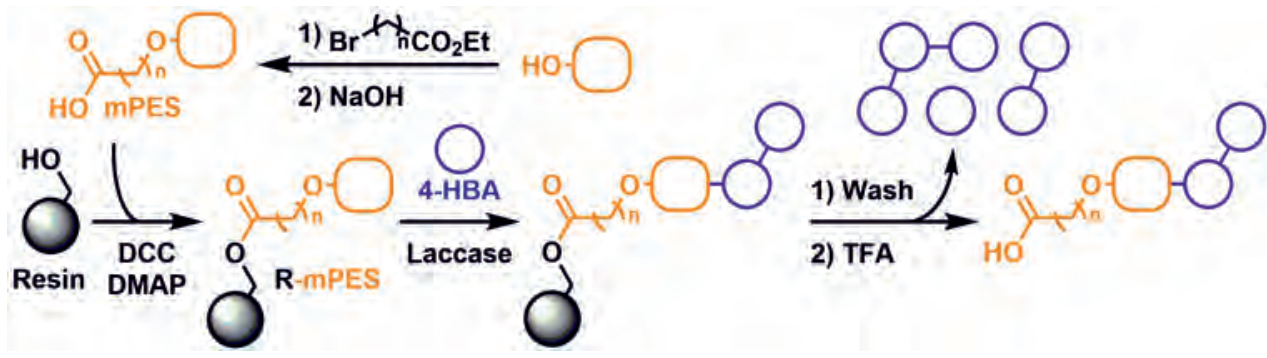

Scheme 5. Schematic overview of the anticipated laccase-mediated oligomerisation of 4-HBA in the presence of resinbound PES model compounds.

Since, to the best of our knowledge, enzymatic transformations on resin-bound model compounds to mimic surface chemistry have not been studied before, this methodology was first verified by studying a well-known reaction: the laccase-mediated oligomerisation of 4-HBA derivatives. Protection of the hydroxy moiety of 4-HBA as a tetrahydropyranyl (THP) ether afforded 4-(OTHP)BA. 4-(OTHP)BA was subsequently coupled to a hydroxy-functionalised NovaPEG Wang resin using $N, N$ dicyclohexylcarbodiimide and $N, N$-dimethylaminopyridine. NovaPEG resins have a backbone entirely 
constituted of poly(ethylene glycol) units (ChemMatrix), allowing for excellent swelling properties in both water and organic solvents. ${ }^{[27]}$ Approximation of reaction completion was accomplished through FTIR analysis, as coupling resulted in the appearance of a distinct carbonyl stretching vibration signal at $\bar{v}=1713 \mathrm{~cm}^{-1}$ corresponding to ester formation (Figure 6). When the intensity of this signal did not intensify any further, the reaction was deemed complete. On-resin deprotection could subsequently be achieved with pyridinium paratoluenesulfonate. Resin-bound 4-HBA was thereafter added to a mixture of laccase and 3-fluoro-4-hydroxybenzoic acid (3-F-4-HBA) and reacted for a short period of time. 3-F4-HBA was chosen as phenol in solution to be able to differentiate solution-phase dimerisation from solid-phase dimerisation; 3-F-4-HBA would form heterogeneous dimers with resin-bound 4-HBA, where it would form homogeneous dimeric 3-F-4-HBA in solution. After laccase-mediated oligomerisation, washing of the resin was followed by trifluoroacetic acid-mediated cleavage.

The cleaved material was compared to oligomerised 4-HBA and oligomerised 3-F-4-HBA by means of LC-MS. The use of 3-F-4-HBA not only introduced a moiety with a distinct mass (because of its fluorine atom), but it also proved to be significantly more hydrophobic than 4-HBA itself, which assured good LC separation. The main products of the laccase-mediated oligomerisation of 4-HBA are two dimers with $m / z 273.04$ ( 1 and 2). ${ }^{[12]}$ Analogously, dimerisation of 3-F-4-HBA was expected to result in a C3-C3'-bound dimer (5) and a C3-O-bound dimer (6), both having a calculated $m / z$ of 309.02. Oligomerisation of 3-F-4-HBA indeed resulted in the formation of two products with $\mathrm{m} / \mathrm{z}$ 309.02 eluting at $6.5 \mathrm{~min}$ (5) and $15.1 \mathrm{~min}$ (6, Figure 7). As expected, corresponding dimeric products of the laccase-mediated oligomerisation of 4-HBA eluted earlier: at $5.1 \mathrm{~min}$ (1) and $11.2 \mathrm{~min}(\mathbf{2})$. Hetero-dimers $\mathbf{3}$ and $\mathbf{4}$ (calculated $m / z$ 291.03), resulting from the coupling of resin-bound 4-HBA and 3-F-4-HBA in solution, were expected to elute halfway. Two peaks, corresponding to a molecule with $m / z$ 291.03, were indeed found. Detectability of the first compound, eluting at $5.9 \mathrm{~min}$, was substantially obscured by the presence of 3-F-4-HBA adducts ( $4.9 \mathrm{~min}$ ), but indeed proved to elute exactly halfway between $\mathbf{1}$ and $\mathbf{5}$ and was therefore designated as $\mathbf{3}$. Formation of $\mathbf{4}$ proved more apparent, as a clear peak, eluting at 13.2 min, was present, again eluting exactly halfway $\mathbf{2}$ and $\mathbf{6}$. The observation of these heterogeneous dimers ( $\mathbf{3}$ and $\mathbf{4}$ ) thereby proved the eligibility of the proposed methodology to perform enzyme-mediated transformations on resin-bound small-molecules.

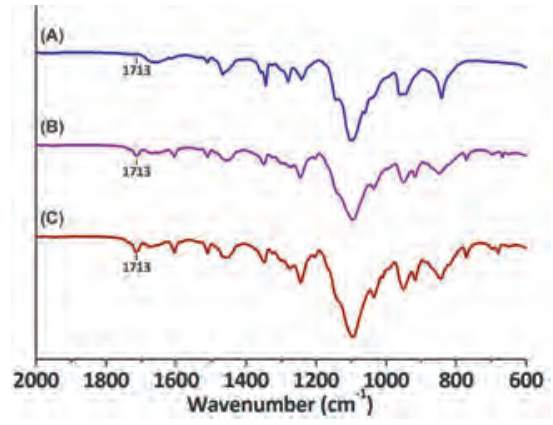

Figure 6. FTIR spectra of A: NovaPEG Wang resin, B: resin-bound 4-(OTHP)BA and C: resinbound 4-HBA.

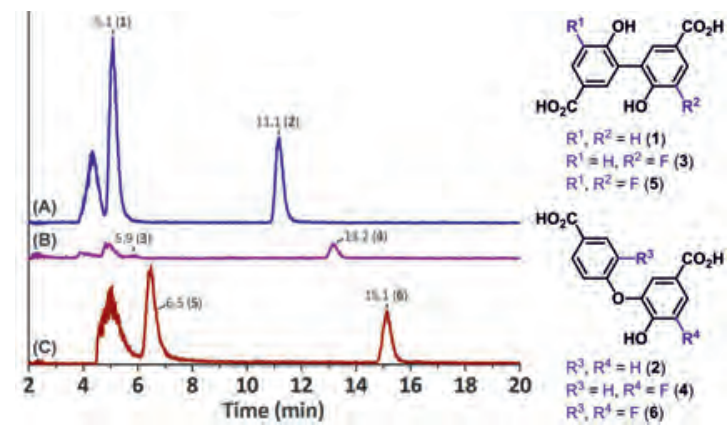

Figure 7. Extracted ion chromatograms of ions whose $m / z$ ([M $\mathrm{H}^{-} \pm 0.50 \mathrm{Da}$ ) corresponds to that of $\mathrm{A}: \mathbf{1}$ and $\mathbf{2}$ after laccasemediated oligomerisation of 4-HBA, B: $\mathbf{3}$ and $\mathbf{4}$ after laccasemediated oligomerisation of 3-F-4-HBA in the presence of resinbound 4-HBA and $\mathrm{C}$ : $\mathbf{5}$ and $\mathbf{6}$ after laccase-mediated oligomerisation of 3-F-4-HBA. Depicted structures of 3, 4, 5 and $\mathbf{6}$ are merely examples of possible isomers.

In order for the PES model compound to be bound to the NovaPEG Wang resin, it had to be functionalised with a carboxylic acid. Therefore, commercially available 4-((4isopropoxyphenyl)sulfonyl)phenol was etherified using ethyl 4-bromobutanoate, followed by hydrolysis of its ethyl ester. Initially, coupling of the PES model compounds was conducted in pure dichloromethane, however, switching to a mixture of dichloromethane and dimethylformamide allowed for more reproducible coupling. The carboxylic acid-functionalised PES model compound ( $\left.\mathrm{mPES}_{3}\right)$ could 
directly be subjected to enzymatic catalysis after coupling it to the resin. Laccase-mediated oligomerisation of 4-HBA in the presence of the resin-bound PES model compound (R-mPES 3 ) was conducted similarly to the oligomerisation of 3-F-4-HBA in the presence of resin-bound 4-HBA. A targeted search for R-mPES $3 / 4-H B A, ~ R-m P E S_{3} / d_{i m 1}$ and R-mPES $/$ dim2 conjugates by means of LC-MS was conducted after cleavage from the resin. However, no conjugates could be observed (Figure 8); the peak observed for molecules with a calculated $m / z$ of 649.14 (corresponding to $\mathrm{mPES}_{3} / 4-\mathrm{HBA}$ ) proved to be an adduct of $\mathrm{MPES}_{3}$ itself as this peak was also observed when $\mathrm{MPES}_{3}$ was individually subjected to LC-MS analysis. Laccase-mediated oligomerisation of the, more reactive, phenols, vanillic acid and syringic acid, in the presence of R-mPES 3 also did not result in conjugate formation (SI Figure S28-S32). As accessibility of the PES model compound might be limited due to immobilisation near the resin's matrix, formation of conjugates could be hampered. The three-carbon spacer was replaced for a ten-carbon spacer to enhance accessibility of the resin-bound PES model compound (R-mPES 10 ). Laccase-mediated oligomerisation of 4-HBA in the presence of R-mPES 10 did, however, not result in covalent bonding between 4-HBA (dimers) and R-mPES 10 either (SI Figure S33). It can therefore be stated that, although laccase-assisted transformations on resin-bound 4-HBA proved to be possible, laccase-mediated conjugation of 4-HBA and resin-bound PES model compounds could not be demonstrated.

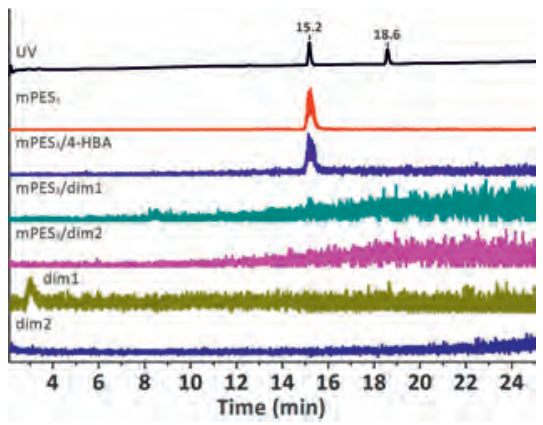

Figure 8. UV trace and extracted ion chromatograms of ions whose $m / z\left(\left[\mathrm{M}-\mathrm{H}^{-} \pm 0.50 \mathrm{Da}\right)\right.$ corresponds to that of $\mathrm{mPES}_{3}, \operatorname{dim} 1 / \operatorname{dim} 2$ or their conjugates after incubation of $4-\mathrm{HBA}$, laccase and R-mPES${ }_{3}$.

\section{Rewriting the mechanism behind the laccase-assisted surface functionalisation of PES}

Although laccase-mediated oligomerisation of 4-HBA proved to be highly effective in inducing antifouling properties to commercially available PES membranes, the mechanism behind the formation of the layer of oligomeric 4-HBA had not been elucidated yet. It was hypothesised that phenolic radicals would bind covalently to PES. We were, however, not able to observe any covalent bonding to the PES membrane or to model compounds mimicking it. Furthermore, the limited amount of charged phenolics present on PES after laccase-mediated oligomerisation of positively charged phenolics could easily be removed by wiping. Therefore, the hypothesis above had to be altered: laccase-assisted surface functionalisation of PES does not result from covalent binding, but rather from strong, but aspecific physical adsorption. This would clarify why the use of 4-HBA results in more surface functionalisation than the use of charged phenolics does; oligomeric 4-HBA precipitates readily and is, due to its low solubility, not easily washed off, whereas oligomers of charged phenols do not, or barely, form a precipitate and are also readily removed by aqueous washing. The use of other phenols, that are known to be oligomerised by laccase, but do not precipitate, is therefore expected not to result in PES membrane modification. Furthermore, extensive washing of PES membranes that have been functionalised with oligomers of 4-HBA should thus result in further removal of these oligomers.

To test whether the use of soluble phenolic oligomers would result in modification of PES, the phenolic monomer guaiacol sulfonate was chosen. Oligomers resulting from the laccase-mediated conversion of guaiacol sulfonate have been shown to be (completely) soluble in acidic aqueous media. ${ }^{[28]}$ It was, analogously, anticipated that this would also be true for oligomers of 4,5-dihydroxy- 
1,3-benzenedisulfonate (Tiron). Oligomerisation of guaiacol sulfonate resulted in a reaction medium being coloured intensely yellow, where oligomerisation of 4,5-dihydroxy-1,3-benzenedisulfonate resulted in a deeply red medium (while both reaction media remained completely transparent by eye). As predicted, the colour of the solution was not transferred to the membranes, which thus indicated that modification did not take place. This was confirmed through XPS analysis: no chemical alterations were observed apart from those resulting from enzyme adsorption (Figure 9).
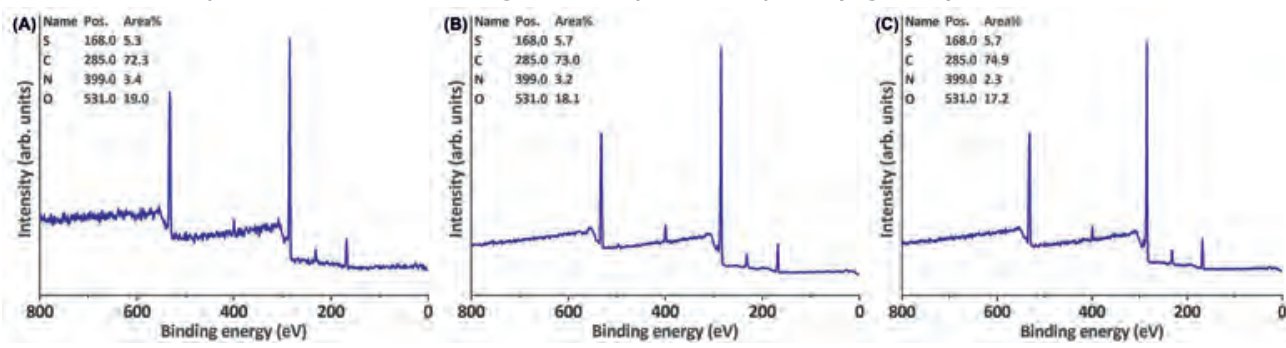

Figure 9. XPS wide scans of a PES membrane after incubation with laccase and A: guaiacol sulfonate or B: 4,5dihydroxy-1,3-benzenedisulfonate. C: XPS wide scan of a blank PES membrane.

Laccase-assisted modification of PES membranes with oligomeric 4-HBA resulted in intense colouration of the modified membrane, which persisted after strong flushing and repeated dipping in water. ${ }^{[9]}$ If modification proves indeed to be non-covalent, more extensive washing should result in less intense membrane colouration. Initial ( $2 \mathrm{~min}$ ) flushing on one side of the membrane with lukewarm tap water resulted in a distinct loss of colouration (Figure 10A) compared to the side that had not been flushed (Figure 10C). Elongating the time of flushing to $5 \mathrm{~min}$ resulted in minimal additional colour loss (Figure 10B and 10D). Where subsequent washing in water ( $40^{\circ} \mathrm{C}, 72 \mathrm{~h}$ ) did not alter membrane colouration (Figure 10G), addition of a detergent (TWEEN ${ }^{\circledR}, 0.1$ wt\%) resulted in a homogeneously tainted membrane of which colouration was significantly less intense than before washing (Figure 10F). As expected, oligomers of 4-HBA are poorly soluble in water and removal could therefore not be achieved by washing with water. However, the fact that washing with a detergent solution resulted in membrane decolouration, confirmed that laccase-assisted coating of PES occurs through adsorption of, rather than through covalently binding phenolic oligomers.

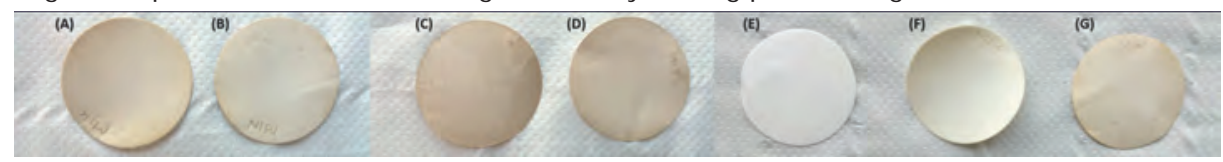

Figure 10. PES membranes that were modified through laccase-mediated oligomerisation of 4-HBA and subsequently cleaned by flushing for A: 2 min or B: 5 min. Backside of membranes of which the front is flushed for C: 2 min or D: 5 min. E: Blank membrane and membranes that have been additionally washed in an aqueous solution of F: TWEEN ${ }^{\circledR}$ $(0.1$ wt $\%)$ or G: water. Image colours have been adjusted as such, that background colouration is similar for all membranes.

\section{Conclusions}

Laccase-mediated oligomerisation of 4-hydroxybenzoic acid (4-HBA) in the presence of PES membranes has been reported to cover the surface with strongly bound coloured material that induced anti-fouling properties to the membrane. ${ }^{[9]}$ Modification was assumed to result from covalent bonding of laccase-generated 4-HBA radicals to the membrane surface. However, the use of a plurality of positively charged phenolics synthesised in-house (instead of 4-HBA) did not, or barely, result in membrane modification. The use of commercially available negatively charged phenolics that, upon oligomerisation, remained completely soluble did not result in modification of the PES surface either. The use of model compounds to mimic a PES surface allowed for easier detection of covalent binding. LC-MS analysis after incubation of either solid, soluble or resin-bound PES model compounds in a mixture of laccase and 4-HBA (derivatives) did not lead to the observation of any covalently bound 
conjugates between the PES model compound and a (oligomeric) 4-HBA (derivative). These observations led us to believe that the mechanism behind laccase-initiated coating of PES membranes occurs through physical adsorption of oligomeric 4-HBA, rather than through binding it to the surface covalently. This hypothesis was supported by the fact that membranes modified through laccaseinitiated modification with oligomers of 4-HBA were significantly less intensely coloured after extensive washing with a detergent solution. Finally, to inform other researchers that laccase-mediated surface functionalisation could easily be interpreted as the result of covalent grafting rather than strong physical adsorption, we recently also reviewed (attempts at) laccase-assisted grafting and assessed to what degree the induced modification could be the result of covalent grafting. ${ }^{[25 b]}$ By doing so, we have identified multiple other cases in which the intended covalent surface modification seems highly unlikely.

\section{Experimental}

\section{Chemicals and general remarks}

2,4,6-tris(Dimethylaminomethyl)phenol (95\%) and laccase from Trametes versicolor (12.9 U mg-1 according to supplier, EC number: 1.10.3.2) were bought from Sigma Aldrich. 4(Trifluoromethoxy)phenol (98\%), 4-((4-isopropoxyphenyl)sulfonyl)phenol (95\%) and $N, N-$ dimethyltyramine (95\%) were obtained from Fluorochem. Disodium 4,5-dihydroxy-1,3benzenedisulfonate (Tiron) monohydrate $(>98 \%)$ and potassium guaiacolsulfonate hemihydrate (>98\%) were supplied by TCl. 4-Hydroxybenzoic acid (99+\%) was bought at Acros Organics. NovaPEG Wang resin (batch 1: $0.62 \mathrm{~mol} \mathrm{~g}^{-1}$, batch 2: $0.46 \mathrm{~mol} \mathrm{~g}^{-1}$ ) was obtained from Merck Millipore. Poly(ethersulfone) membranes $(0.2 \mu \mathrm{m}$ pores, $50 \mathrm{~mm}$ diameter) were bought at Sartorius Stedim. Poly(ethersulfone) sheets $(10 \times 10 \mathrm{~mm})$ were obtained from Goodfellow.

For the sake of consistency, numbering of phenol carbon atoms was based on that of 4hydroxybenzoic acid. Concerning solid-phase chemistry, equivalents or amounts of mol in quotation marks ("') ) are based on full loading of the resin. Flash column chromatography was conducted using flash silica gel $60 .{ }^{1} \mathrm{H}$ and ${ }^{13} \mathrm{C}$ NMR spectra were either recorded on a $400 \mathrm{MHz}$ Bruker Avance spectrometer or a $600 \mathrm{MHz}$ Avance III spectrometer equipped with a cryo-probe. All signals were referenced relative to the residual solvent signal and coupling constants / are given in $\mathrm{Hz}$. Highresolution mass spectra were recorded on a Thermo Scientific Exactive instrument. Prior to modification, PES membranes were flushed with water and PES sheets were sonicated in methanol. All MS chromatograms were normalised to the peak with highest intensity. Extracted ion chromatograms are always displayed as a plot of $\left[\mathrm{M}-\mathrm{H}^{-} \pm 0.50 \mathrm{Da}\right.$.

\section{Synthetic procedures}

tert-Butyl 4-hydroxybenzoate (E1).[29] 4-Hydroxybenzoic acid $(5.0 \mathrm{~g}, 36.2 \mathrm{mmol}, 1$ eq.), tertbutanol ( $70 \mathrm{ml}, 0.72 \mathrm{~mol}, 20$ eq.) and $N, N$-dimethyl-4-aminopyridine (DMAP, $180 \mathrm{mg}, 1.45 \mathrm{mmol}, 0.04$ eq.) were dissolved in anhydrous tetrahydrofuran ( $120 \mathrm{ml}$ ) in a flame-dried round-bottom flask under argon. A solution of $\mathrm{N}, \mathrm{N}$-dicyclohexylcarbodiimide (DCC, $11.2 \mathrm{~g}, 54.3 \mathrm{mmol}, 1.5 \mathrm{eq}$.) in anhydrous tetrahydrofuran $(50 \mathrm{ml})$ was subsequently added dropwise over the course of $30 \mathrm{~min}$. This mixture was thereafter stirred for $20 \mathrm{~h}$ under an argon atmosphere at room temperature and subsequently filtered. Succeeding concentration in vacuo, the filtrate was washed with a $1 \mathrm{M}$ aqueous sodium bicarbonate solution $(50 \mathrm{ml})$ and thereafter purified using flash chromatography (elution from $0 \%$ to $30 \%$ ethyl acetate in petroleum ether 40-60). The final product (E1) was yielded as a white solid (37\%, $2.61 \mathrm{~g}, 13.4 \mathrm{mmol}) . \delta_{\mathrm{H}}\left(400 \mathrm{MHz}, \mathrm{CDCl}_{3}\right) 7.93-7.86(2 \mathrm{H}, \mathrm{m}, \mathrm{ArH}), 6.88-6.81(2 \mathrm{H}, \mathrm{m}, \mathrm{ArH})$, $1.58\left(9 \mathrm{H}, \mathrm{S}, \mathrm{CH}_{3}\right) . \delta \mathrm{c}\left(101 \mathrm{MHz} \mathrm{CDCl}_{3}\right) 166.4,160.1,131.7,124.0,115.1,81.1,28.3$. HRMS (ESI) $m / z$ [M - H] 193.0870 (calcd. 193.0870). 
General procedure for Mannich reaction. ${ }^{[21]}$ The respective para-substituted phenol (B1-E1, 3.0 mmol, 1.0 eq.) was dissolved in ethanol $(10 \mathrm{ml})$ under argon, after which dimethylamine ( $40 \mathrm{wt} \%$ in water, $1.1 \mathrm{ml}, 9.0 \mathrm{mmol}, 3.0$ eq.) was added. Careful dropwise addition of formaldehyde (37 wt\% in water, $0.58 \mathrm{ml}, 7.8 \mathrm{mmol}, 2.6 \mathrm{eq}$.) to this mixture was followed by refluxing overnight. The volatiles were evaporated under reduced pressure after which the concentrated residue was diluted with diethyl ether $(20 \mathrm{ml})$ and washed with a saturated aqueous sodium carbonate solution ( $20 \mathrm{ml}$ ) before being extracted with diethyl ether ( $3 \times 20 \mathrm{ml}$; to minimise yellowing, the extracts were intermittently purged with argon). Subsequent to evaporation of all volatiles, the crude product (B2-E2) was sufficiently pure to start follow-up chemistry, and was stored between 2 to $7^{\circ} \mathrm{C}$ under argon prior to further use.

General procedure for methylation. The respective Mannich base (B2-F2, $2.6 \mathrm{mmol}, 1$ eq.) was dissolved in anhydrous acetonitrile (15 ml) in a flame-dried round-bottom flask under argon, after which methyl iodide $(0.97 \mathrm{ml}, 15.6 \mathrm{mmol}, 6$ eq.) was added dropwise. Subsequent to being stirred at room temperature for $48 \mathrm{~h}$, a second portion of methyl iodide $(0.97 \mathrm{ml}, 15.6 \mathrm{mmol}, 6$ eq. $)$ was added dropwise and stirring was continued for another $24 \mathrm{~h}$. After filtration, the product (B3-F3) was used in the subsequent step without further purification.

1,1'-(5-Carboxy-2-hydroxy-1,3-phenylene) bis( $N, N, N$-trimethylmethanaminium) diiodide (E4). 1,1'-(5-(tert-Butoxycarbonyl)-2-hydroxy-1,3-phenylene) bis( $N, N, N$-trimethylmethanaminium) diiodide (E3, $200 \mathrm{mg}, 0.34 \mathrm{mmol}, 1$ eq.) was suspended in acetonitrile (3 ml) after which trifluoroacetic acid ( $3 \mathrm{ml}, 40 \mathrm{mmol},>100$ eq.) was added dropwise. The suspension was stirred at room temperature for $3 \mathrm{~h}$, after which the remaining solid (E4) was concentrated in vacuo and used in the next step without further purification.

General I/ Cl ion exchange procedure. Dowex Cl-form ion exchange resin (1X8-200) was washed and wetted with a 1:1 mixture of methanol/water (1:3 $V W$ washing solvent/resin). The resin was further rinsed with water to wash off all methanol and the wet resin was isolated by filtration. The respective diiodide salt (B3-D3, E4 or F3) and wet resin (10 g per g diiodide salt) were then added to water ( $20 \mathrm{ml}$ per $\mathrm{g}$ diiodide salt) and stirred gently at room temperature for $1 \mathrm{~h}$. Subsequent removal of the resin through filtration and evaporation of water yielded the white/off-white product.

$\mathbf{N}, \mathbf{N}, \mathbf{N}$-Trimethyltyramine chloride (A). $N, N$-Dimethyltyramine (400 mg, $2.42 \mathrm{mmol}, 1$ eq.) was dissolved in anhydrous acetonitrile (16 ml) in a flame-dried round-bottom flask under argon, after which methyl iodide $(0.97 \mathrm{ml}, 15.6 \mathrm{mmol}, 6$ eq.) was added dropwise. Subsequent to rapid emergence of a precipitate, the mixture was stirred at room temperature for $24 \mathrm{~h}$. The mixture was thereafter cooled on ice and diluted with ice-cold diethyl ether. Filtration afforded the product as a tanned white powder which was used in the next step without further purification. Dowex Cl-form ion exchange resin (1X8-200) was washed and wetted with a 1:1 mixture of methanol/water (1:3 $V W$ washing solvent/resin). The resin was further rinsed with water to wash off all methanol and the wet resin was isolated by filtration. $N, N, N$-Trimethyltyramine iodide $(690 \mathrm{mg}, 2.25 \mathrm{mmol})$ and wet resin $(8 \mathrm{~g})$ were then added to water $(30 \mathrm{ml}$ ) and stirred gently at room temperature for $1 \mathrm{~h}$. After filtering off the resin and evaporation of water, the resulting $\mathrm{N}, \mathrm{N}, \mathrm{N}$-trimethyltyramine chloride (A) was isolated as a white solid (96\% over two steps, $502 \mathrm{mg}, 2.33 \mathrm{mmol}) . \delta_{\mathrm{H}}\left(400 \mathrm{MHz}, \mathrm{D}_{2} \mathrm{O}\right) 7.32-7.13(2 \mathrm{H}, \mathrm{m}, \mathrm{ArH}), 6.98-$ $6.83(2 \mathrm{H}, \mathrm{m}, \mathrm{ArH}), 3.60-3.42\left(2 \mathrm{H}, \mathrm{m}, \mathrm{CH}_{2}\right), 3.17\left(9 \mathrm{H}, \mathrm{s}, \mathrm{NCH}_{3}\right), 3.12-3.03\left(2 \mathrm{H}, \mathrm{m}, \mathrm{CH}_{2}\right) . \delta \mathrm{c}(101$ $\mathrm{MHz}, \mathrm{D}_{2} \mathrm{O}$ ) 154.5, 130.3, 127.4, 115.8, 67.2 (t), 52.9 (t), 27.9. HRMS (ESI) $m / z$ [M] 180.1381 (calcd. 180.1383).

\section{1,1'-( 2-Hydroxy-5-methoxy-1,3-phenylene) bis( $N, N, N$-trimethylmethanaminium)}

dichloride (B). Off-white solid, $13 \%$ over three steps. $\delta_{\mathrm{H}}\left(400 \mathrm{MHz}, \mathrm{D}_{2} \mathrm{O}\right) 7.27(2 \mathrm{H}, \mathrm{s}, \mathrm{ArH}), 4.60$ (4 
$\left.\mathrm{H}, \mathrm{s}, \mathrm{CH}_{2}\right), 3.87\left(3 \mathrm{H}, \mathrm{s}, \mathrm{OCH}_{3}\right), 3.16\left(18 \mathrm{H}, \mathrm{s}, \mathrm{NCH}_{3}\right) . \delta \mathrm{c}\left(101 \mathrm{MHz}, \mathrm{D}_{2} \mathrm{O}\right) 153.1,150.0,122.7,119.8$, 64.1, 56.2, 52.7. HRMS (ESI) $m / z[\mathrm{M}]^{2+} 134.1064$ (calcd. 134.1070).

\section{1,1'-(2-Hydroxy-5-( methoxycarbonyl)-1,3-phenylene) bis( $N, N, N$}

trimethylmethanaminium) dichloride (C). White solid, $38 \%$ over three steps. $\delta_{\mathrm{H}}\left(400 \mathrm{MHz}, \mathrm{D}_{2} \mathrm{O}\right)$ $8.22(2 \mathrm{H}, \mathrm{s}, \mathrm{ArH}), 4.62\left(4 \mathrm{H}, \mathrm{s}, \mathrm{CH}_{2}\right), 3.93\left(3 \mathrm{H}, \mathrm{s}, \mathrm{OCH}_{3}\right), 3.14\left(18 \mathrm{H}, \mathrm{s}, \mathrm{NCH}_{3}\right) . \delta \mathrm{c}\left(101 \mathrm{MHz}, \mathrm{D}_{2} \mathrm{O}\right)$ 167.9, 164.3, 138.8, 120.0, 118.0, 64.1, 52.6, 52.5. HRMS (ESI) $m / z[M]^{2+} 148.1043$ (calcd. 148.1045).

\section{1,1'-(2-Hydroxy-5-(trifluoromethoxy)-1,3-phenylene) bis( $N, N, N-$}

trimethylmethanaminium) dichloride (D). Additionally purified through preparative HPLC. White solid, < 1\% over three steps. $\delta \mathrm{H}\left(400 \mathrm{MHz}, \mathrm{D}_{2} \mathrm{O}\right) 7.61(2 \mathrm{H}, \mathrm{s}, \mathrm{ArH}), 4.58\left(4 \mathrm{H}, \mathrm{s}, \mathrm{CH}_{2}\right), 3.13(16 \mathrm{H}, \mathrm{s}$, $\left.\mathrm{NCH}_{3}\right) . \delta c\left(151 \mathrm{MHz}, \mathrm{D}_{2} \mathrm{O}\right) 158.5,140.1,130.3,119.3,64.0,52.6$. HRMS (ESI) $\mathrm{m} / z[\mathrm{M}]^{2+} 161.0927$ (calcd. 161.0929).

1,1'-(5-Carboxy-2-hydroxy-1,3-phenylene) bis( $N, N, N$-trimethylmethanaminium) dichloride (E). White solid, $20 \%$ over four steps. $\delta_{\mathrm{H}}\left(400 \mathrm{MHz}, \mathrm{D}_{2} \mathrm{O}\right) 8.27(2 \mathrm{H}, \mathrm{s}, \mathrm{ArH}), 4.68\left(4 \mathrm{H}, \mathrm{s}, \mathrm{CH}_{2}\right), 3.15$ (18 H, S, NCH $)$. $\delta c\left(101 \mathrm{MHz}, \mathrm{D}_{2} \mathrm{O}\right)$ 168.6, 160.9, 139.1, 123.0, 118.0, 63.6, 52.6. HRMS (ESI) $m / z[M]^{2+} 141.0965$ (calcd. 141.0966).

1,1',1'-(2-Hydroxybenzene-1,3,5-triyl)tris( $N, N, N$-trimethylmethanaminium) trichloride (F). Additionally purified through precipitation from methanol and preparative HPLC. White solid, 16\% over two steps. $\delta_{\mathrm{H}}\left(400 \mathrm{MHz}, \mathrm{D}_{2} \mathrm{O}\right) 7.66(2 \mathrm{H}, \mathrm{s}, \mathrm{ArH}), 4.55(4 \mathrm{H}, \mathrm{s} \mathrm{CH}), 4.45\left(2 \mathrm{H}, \mathrm{s}, \mathrm{CH}_{2}\right), 3.12$ (18 $\left.\mathrm{H}, \mathrm{s}, \mathrm{NCH}_{3}\right), 3.10\left(9 \mathrm{H}, \mathrm{s}, \mathrm{NCH}_{3}\right) . \delta \mathrm{c}\left(101 \mathrm{MHz}, \mathrm{D}_{2} \mathrm{O}\right) 165.9,141.4,118.8,114.3,68.9,64.7,52.5$ (t), 51.9 (t). HRMS (ESI) $m / z[M]^{3+} 103.4283$ (calcd. 103.4281).

4,4'-Sulfonylbis(methoxybenzene) (G). 4,4'-Sulfonyldiphenol ( $2.5 \mathrm{~g}, 9.90 \mathrm{mmol}, 1$ eq.) was dissolved in dimethylformamide ( $120 \mathrm{ml}$ ) after which potassium carbonate $(6.9 \mathrm{~g}, 49.9 \mathrm{mmol}, 5 \mathrm{eq}$.) and methyl iodide $(9.3 \mathrm{ml}, 149 \mathrm{mmol}, 15$ eq.) were added. This mixture was stirred at room temperature for $48 \mathrm{~h}$ and subsequently filtered over Celite and washed with dichloromethane. Volatiles (including dimethylformamide) were removed in vacuo, after which the concentrate was diluted with ethyl acetate $(150 \mathrm{ml})$ and subsequently washed with water $(100 \mathrm{ml})$. The aqueous phase was extracted with ethyl acetate $(2 \times 50 \mathrm{ml})$ and the combined organic phases were subsequently washed with $5 \%$ aqueous sodium metabisulfite $(2 \times 50 \mathrm{ml})$, saturated aqueous sodium bicarbonate $(2 \times 20 \mathrm{ml})$ and brine $(100 \mathrm{ml})$. Evaporation of all volatiles afforded $\mathbf{G}$ as a white solid $(87 \%, 2.41 \mathrm{~g}, 8.66 \mathrm{mmol})$. $\delta \mathrm{H}\left(400 \mathrm{MHz}, \mathrm{CDCl}_{3}\right) 7.83\left(4 \mathrm{H}, \mathrm{d}, \int\right.$ 8.7, $\left.\mathrm{ArH}\right), 6.93(4 \mathrm{H}, \mathrm{d}$, J 8.7, $\mathrm{ArH}), 3.82\left(6 \mathrm{H}, \mathrm{s}, \mathrm{CH}_{3}\right) . \delta \mathrm{c}(101$ $\mathrm{MHz}, \mathrm{CDCl}_{3}$ ) 163.1, 133.9, 129.5, 114.4, 55.6. HRMS (ESI) $\mathrm{m} / z[\mathrm{M}+\mathrm{H}]^{+} 279.0681$ (calcd. 279.0686).

2,2'-((Sulfonylbis(4,1-phenylene)) bis(oxy)) diacetic acid (H). 4,4'-Sulfonyldiphenol (2.0 g, 8.00 mmol, 1 eq.) was dissolved in dimethylformamide $(20 \mathrm{ml})$ after which potassium carbonate $(1.7 \mathrm{~g}$, $12.0 \mathrm{mml}, 1.5$ eq.) and ethyl chloroacetate $(1.7 \mathrm{ml}, 16.0 \mathrm{mmol}, 2$ eq.) were added. This mixture was stirred at $100{ }^{\circ} \mathrm{C}$ for $3 \mathrm{~h}$, cooled to $\mathrm{rt}$ and subsequently diluted with water $(50 \mathrm{ml}$ ) before being extracted with ethyl acetate $(3 \times 50 \mathrm{ml})$. The intermediate ethyl ester was concentrated in vacuo and used without further purification in the next step by dissolving it $(1.9 \mathrm{~g}, 4.49 \mathrm{mmol}, 1 \mathrm{eq}$.) in methanol $(20 \mathrm{ml})$ and cooling the solution down to $0{ }^{\circ} \mathrm{C}$. After addition of a $1 \mathrm{M}$ aqueous solution of sodium hydroxide (11 ml, $11.0 \mathrm{mmol}, 2.5$ eq.) the mixture was stirred at room temperature for $2 \mathrm{~h}$. Concentration in vacuo was followed by dilution with a $1 \mathrm{M}$ aqueous solution of hydrochloric acid (13 $\mathrm{ml}, 13.0 \mathrm{mmol}, 3$ eq.). Extraction with ethyl acetate $(3 \times 50 \mathrm{ml}$ ), washing of the combined organic layers with brine followed by concentration in vacuo afforded $\mathbf{H}$ as a white solid ( $63 \%$ over two steps, $1.34 \mathrm{~g}, 3.66 \mathrm{mmol}$ ). $\delta_{\mathrm{H}}(400 \mathrm{MHz}, \mathrm{MeOD}) 7.85$ (4 H, d, J 8.8, ArH), 7.07 (4 H, d, J 8.8, ArH), 4.75 (4 
$\left.\mathrm{H}, \mathrm{S}, \mathrm{CH}_{2}\right) . \delta \mathrm{c}(101 \mathrm{MHz}, \mathrm{MeOD}) 171.7,163.2,135.7,130.6,116.3,65.8$. HRMS (ESI) $m / z[\mathrm{M}-\mathrm{H}]$ 365.0334 (calcd. 365.0337).

4-((Tetrahydro-2Hpyran-2-yl)oxy) benzoic acid (4-(OTHP)BA) . ${ }^{\text {[30] }}$ 4-Hydroxybenzoic acid (2.0 g, $14.5 \mathrm{mmol}, 1$ eq.), 2,3-dihydropyran (6.6 ml, $72.4 \mathrm{mmol}, 5$ eq.) and paratoluenesulfonic acid (catalytic amount) were stirred in anhydrous tetrahydrofuran (10 $\mathrm{ml}$ ) in a flame-dried round-bottom flask under nitrogen at room temperature for $5 \mathrm{~h}$. After addition of a saturated aqueous sodium bicarbonate solution $(20 \mathrm{ml})$ and subsequent extraction with ethyl acetate $(3 \times 20 \mathrm{ml})$ the product was yielded as a white solid $(38 \%, 1.2 \mathrm{~g}, 5.58 \mathrm{mmol}) . \delta_{\mathrm{H}}\left(400 \mathrm{MHz}, \mathrm{CDCl}_{3}\right) 8.09-8.03(2 \mathrm{H}, \mathrm{m}, \mathrm{ArH}), 7.13$ - $7.07(2 \mathrm{H}, \mathrm{m}, \mathrm{ArH}), 5.53(1 \mathrm{H}, \mathrm{t}$, J 3.1, CH), $3.87(1 \mathrm{H}, \mathrm{ddd}$, J 11.2, 10.1, 3.1, C $(H) \mathrm{H}), 3.68$ - 3.59 (1 $\mathrm{H}, \mathrm{m}, \mathrm{C}(H) \mathrm{H}), 2.09-1.97(1 \mathrm{H}, \mathrm{m}, \mathrm{C}(H) \mathrm{H}), 1.89\left(2 \mathrm{H}, \mathrm{dt}\right.$, J 7.6, 3.7, $\left.\mathrm{CH}_{2}\right), 1.79-1.65(2 \mathrm{H}, \mathrm{m}, 2 \times$ $\mathrm{C}(H) \mathrm{H}), 1.65-1.57(1 \mathrm{H}, \mathrm{m}, \mathrm{C}(H) \mathrm{H}) . \delta \mathrm{c}\left(101 \mathrm{MHz}, \mathrm{CDCl}_{3}\right) 171.8,161.6,132.2,122.3,116.0,96.1$, 62.1, 30.1, 25.1, 18.5. HRMS (ESI) $m / z$ [M - H]- 221.0819 (calcd. 221.0821).

4-(4-((4-I sopropoxyphenyl)sulfonyl)phenoxy)butanoic acid (mPES 3$)$. A mixture of 4-((4isopropoxyphenyl)sulfonyl)phenol (500 mg, $1.71 \mathrm{mmol}, 1$ eq.) and potassium carbonate ( $355 \mathrm{mg}, 2.57$ mmol, 1.5 eq.) in anhydrous butanone ( $10 \mathrm{ml}$ ) was refluxed under nitrogen for $1 \mathrm{~h}$, after which ethyl 4-bromobutanoate (501 mg, $2.57 \mathrm{mmol}, 1.5 \mathrm{eq}$.) was added, and this mixture was further refluxed overnight. After addition of water and extraction with ethyl acetate ( $3 \times 20 \mathrm{ml})$ the intermediate ester was concentrated in vacuo and used in the next step without further purification. The intermediate ester was dissolved in ethanol $(10 \mathrm{ml})$ and the mixture was cooled to $0{ }^{\circ} \mathrm{C}$ before addition of sodium hydroxide ( $137 \mathrm{mg}, 3.42 \mathrm{mmol}$ ). After being stirred at room temperature for $6 \mathrm{~h}$, the reaction mixture was neutralised by addition of saturated aqueous hydrochloric acid $(0.13 \mathrm{ml}, 4.28 \mathrm{mmol})$. Extraction with ethyl acetate $(3 \times 20 \mathrm{ml})$ and evaporation of all volatiles afforded the product as a yellowish solid (90\% over 2 steps, $580 \mathrm{mg}, 1.54 \mathrm{mmol}) . \delta_{\mathrm{H}}\left(400 \mathrm{MHz}, \mathrm{CDCl}_{3}\right) 7.82(4 \mathrm{H}, \mathrm{t}, J$ 9.5, $\mathrm{ArH}), 6.91(4 \mathrm{H}, \mathrm{t}, J$ 9.1, ArH), $4.59\left(1 \mathrm{H}\right.$, hept, J 6.0, CH), $4.04\left(2 \mathrm{H}, \mathrm{t}\right.$, J 6.0, $\left.\mathrm{CH}_{2}\right), 2.56\left(2 \mathrm{H}, \mathrm{t}\right.$, J 7.2, $\left.\mathrm{CH}_{2}\right), 2.12(2 \mathrm{H}, \mathrm{q}$, J 6.5, $\left.\mathrm{CH}_{2}\right), 1.33\left(6 \mathrm{H}, \mathrm{d}\right.$, J 6.1, $\left.\mathrm{CH}_{3}\right) . \delta \mathrm{c}\left(101 \mathrm{MHz}, \mathrm{CDCl}_{3}\right)$ 178.6, 162.2, 161.6, 134.2, 133.2, 129.5, $115.7,114.8,70.4,66.9,30.2,24.1,21.8$. HRMS (ESI) $m / z$ [M - H]- 377.1064 (calcd. 377.1064).

11-(4-((4-I sopropoxyphenyl)sulfonyl)phenoxy) undecanoic acid ( PEES $\left._{10}\right)$. A mixture of 4((4-isopropoxyphenyl)sulfonyl)phenol ( $1.00 \mathrm{~g}, 3.42 \mathrm{mmol}, 1 \mathrm{eq}$ ) and potassium carbonate $(615 \mathrm{mg}$, $4.45 \mathrm{mmol}, 1.3$ eq.) in anhydrous butanone ( $20 \mathrm{ml}$ ) was refluxed under nitrogen for $1 \mathrm{~h}$, after which ethyl 11-bromoundecanoate (1.30 g, $4.45 \mathrm{mmol}, 1.3$ eq.) was added and this mixture was further refluxed overnight. After addition of water and extraction with ethyl acetate ( $3 \times 20 \mathrm{ml})$, the intermediate ester was concentrated in vacuo and used in the next step without further purification. The intermediate ester $(1.00 \mathrm{~g}, 1.98 \mathrm{mmol}, 1 \mathrm{eq}$.) was dissolved in ethanol (10 ml) and the mixture was cooled to $0{ }^{\circ} \mathrm{C}$ before addition of sodium hydroxide ( $137 \mathrm{mg}, 3.42 \mathrm{mmol}, 1.7$ eq.). The reaction mixture had been stirred at room temperature for $6 \mathrm{~h}$, after which it was concentrated in vacuo. Addition of ethyl acetate $(20 \mathrm{ml})$ and aqueous hydrochloric acid $(1 \mathrm{M}, 20 \mathrm{ml})$ followed by extraction with ethyl acetate $(3 \times 20 \mathrm{ml})$ and evaporation of all volatiles afforded the product as a white solid (79\% over 2 steps, $842 \mathrm{mg}, 1.72 \mathrm{mmol}) . \delta \mathrm{H}\left(400 \mathrm{MHz}, \mathrm{CDCl}_{3}\right) 7.81(4 \mathrm{H}, \mathrm{dd}, J$ 8.5, 6.5, ArH), 6.91 (4 $\mathrm{H}, \mathrm{t}$, J 9.4, ArH), $4.58\left(1 \mathrm{H}\right.$, hept, / 6.0, CH), $3.96\left(2 \mathrm{H}, \mathrm{t}\right.$, J 6.5, $\left.\mathrm{CH}_{2}\right), 2.33\left(2 \mathrm{H}, \mathrm{t}\right.$, / 7.5, $\left.\mathrm{CH}_{2}\right), 1.75$ (2 $\mathrm{H}, \mathrm{p}$, J 6.6, $\left.\mathrm{CH}_{2}\right), 1.61\left(2 \mathrm{H}, \mathrm{p}, \boldsymbol{J} 7.2, \mathrm{CH}_{2}\right), 1.45-1.36\left(2 \mathrm{H}, \mathrm{m}, \mathrm{CH}_{2}\right), 1.36-1.25\left(16 \mathrm{H}, \mathrm{m}, \mathrm{CH}_{2}\right.$ \& $\left.\mathrm{CH}_{3}\right)$. $\delta \mathrm{c}\left(101 \mathrm{MHz}, \mathrm{CDCl}_{3}\right)$ 180.0, 162.7, 161.6, 133.7, 133.4, 129.5, 129.5, 115.7, 114.8, 70.4, 68.4, 34.0, 29.4, 29.3, 29.2, 29.2, 29.0, 29.0, 25.9, 24.6, 21.8. HRMS (ESI) $m / z$ [M - H]- 475.2157 (calcd. 475.2160).

\section{Solid-phase chemistry}

Coupling of 4-((tetrahydro-2Hpyran-2-yl)oxy) benzoic acid. NovaPEG Wang Resin (100 mg, "0.06 mmol", "1 eq.", $0.62 \mathrm{mmol} \mathrm{g}^{-1}$ loading) was swollen in anhydrous dichloromethane for $4 \mathrm{~h}$, after 
which 4-((tetrahydro-2H-pyran-2-yl)oxy)benzoic acid (34 mg, $0.15 \mathrm{mmol}, 2.5$ eq.), $N, N$ dicyclohexylcarbodiimide (44 mg, $0.21 \mathrm{mmol}, 3.5$ eq.) and $N, N$-dimethyl-4-aminopyridine (4 mg, 0.03 $\mathrm{mmol}, 0.5$ eq.) were added. The mixture was subsequently stirred gently under a nitrogen atmosphere at $40{ }^{\circ} \mathrm{C}$ for $5 \mathrm{~h}$. The resin was thereafter consecutively washed with methanol and dichloromethane (2x).

Deprotection of resin-bound 4-((tetrahydro-2Hpyran-2-yl)oxy)benzoic acid. Resin-bound 4-((tetrahydro-2H-pyran-2-yl)oxy)benzoic acid (100 mg,"0.06 mmol", 1 eq.) was swollen in dichloromethane for $1 \mathrm{~h}$, after which a solution of pyridinium paratoluenesulfonate (catalytic amount) in methanol was added. The resulting mixture was gently stirred overnight under a nitrogen atmosphere at room temperature. The resin was thereafter consecutively washed with methanol and dichloromethane $(2 x)$.

Coupling of mPES 3 . NovaPEG Wang Resin (565 mg,"0.26 mmol", "1 eq.", 0.46 mmol g-1 loading) was swollen in a mixture of anhydrous dichloromethane $(18 \mathrm{ml})$ and dimethylformamide $(2 \mathrm{ml})$ for $1 \mathrm{~h}$. Addition of $\mathrm{mPES}_{3}(500 \mathrm{mg}, 1.32 \mathrm{mmol}, 5$ eq.), $N, N$-dicyclohexylcarbodiimide ( $161 \mathrm{mg}, 0.78 \mathrm{mmol}, 3$ eq.) and $N, N$-dimethyl-4-aminopyridine $(6 \mathrm{mg}, 0.05 \mathrm{mmol}, 0.2 \mathrm{eq})$ was followed by gentle overnight stirring under a nitrogen atmosphere at room temperature. The resin was thereafter consecutively washed with methanol and dichloromethane $(2 \times)$.

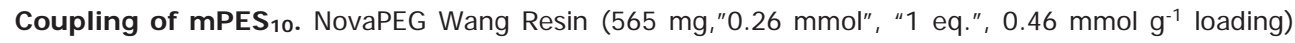
was swollen in a mixture of anhydrous dichloromethane $(18 \mathrm{ml})$ and dimethylformamide $(2 \mathrm{ml})$ for 30 min. Addition of PPES$_{10}$ ( $743 \mathrm{mg}, 1.56 \mathrm{mmol}, 6 \mathrm{eq}$.), $N, N$-dicyclohexylcarbodiimide (161 mg, 0.78 mmol, 3 eq.) and $N, N$-dimethyl-4-aminopyridine (DMAP, $6 \mathrm{mg}, 0.05 \mathrm{mmol}, 0.2 \mathrm{eq}$ ) was followed by gentle overnight stirring under a nitrogen atmosphere at room temperature. The resin was thereafter consecutively washed with methanol and dichloromethane $(2 \times)$.

\section{Laccase purification}

Desolvation and subsequent filtration (GP $0.22 \mu \mathrm{m}$ filter unit, Sterivex ${ }^{\mathrm{TM}}$ ) of commercially available laccase from Trametes versicolor (EC number: 1.10.3.2) ensured removal of large particulates. Initial purification was achieved by means of ion exchange column chromatography (Source 15Q (XK $16 \mathrm{~mm}$ $\times 12 \mathrm{~cm})$, ÄKTA explorer chromatography system). Gradient elution $\left(2 \mathrm{ml} \mathrm{min}{ }^{-1}\right)$ from $0 \mathrm{M}$ sodium chloride in $20 \mathrm{mM}$ tris $\mathrm{HCl}$ buffer ( $\mathrm{pH}$ 7) to $1 \mathrm{M}$ sodium chloride in $20 \mathrm{mM}$ tris $\mathrm{HCl}$ buffer (pH 7) over 20 column volumes was followed by fraction selection based on a $N, N$-diethyl-p-phenylenediamine sulfate assay. Selected fractions were pooled and concentrated to approximately $2 \mathrm{ml}$ using a $10 \mathrm{kDa}$ Amicon ultrafiltration system. Further purification was achieved through gel filtration chromatography (Sephacryl S300 (XK hiload $26 \mathrm{~mm} \times 60 \mathrm{~cm}$ ), ÄKTA explorer chromatography system). Elution with a $0.1 \mathrm{M}$ sodium acetate/acetic acid buffer $(\mathrm{pH} 6)$ afforded fractions that were selected based on a 2,2'azino-bis(3-ethylbenzothiazoline-6-sulphonic acid) (ABTS) diammonium salt assay, pooled and concentrated using a $10 \mathrm{kDa}$ Amicon ultrafiltration system. Concentrated laccase fractions proved to contain $6.6 \mathrm{mg} \mathrm{ml}^{-1}$ of protein as based on a Pierce BCA protein assay with bovine serum albumin as standard. Laccase purity was assessed using SDS-PAGE gel chromatography ( $12 \%$ acrylamide, ESI Fig. S34).

\section{Laccase activity assay}

The specific activity of both commercially available and purified laccase from Trametes versicolor (EC number: 1.10.3.2) was determined spectroscopically using a UV/Vis spectrometer (Varian Cary 50 Scan) by measuring the increase of absorbance for the oxidation of ABTS to the ABTS radical cation ( $\varepsilon$ $=36000$ ). ABTS diammonium salt was dissolved in an aqueous sodium acetate/acetic acid buffer (0.1 
$\mathrm{M}, \mathrm{pH}$ 5), to which laccase was added. The subsequent increase of the optical absorbance at $\lambda=420$ $\mathrm{nm}$ was followed for $60 \mathrm{~s}$.

\section{Laccase-mediated oligomerisation of phenolics in the presence of model compounds} Laccase-mediated oligomerisation of 4-HBA in the presence of G. A solution of 4-HBA (0.1 $\mathrm{mg}, 0.8 \mu \mathrm{mol}, 14.5 \mathrm{mM}$ final concentration, 0.004 eq.) and laccase $\left(0.23 \mathrm{U}, 4.5 \mathrm{U} \mathrm{ml}^{-1}\right)$ in an ammonium acetate/acetic acid buffer ( $100 \mu \mathrm{l}, 0.1 \mathrm{M}, \mathrm{pH}$ 5) was deposited on crystals of PES model compound $\mathbf{G}$ ( $50 \mathrm{mg}, 0.18 \mathrm{mmol}, 1 \mathrm{eq}$.) and allowed to react at it for $24 \mathrm{~h}$.

Work-up: laccase was removed from the reaction mixture using a $9 \mathrm{kDa}$ protein concentrator by employing centrifugal force (Hermle Z 206A). The reaction medium was appropriately diluted with water and analysed by LC-MS (LC-method LC3). The solids were dissolved in ethyl acetate, washed with water and the combined aqueous phases were thereafter extracted with fresh ethyl acetate. After evaporation of ethyl acetate, the extracts were dissolved in methanol and diluted with water to be analysed by LC-MS (LC-method LC3).

Laccase-mediated oligomerisation in the presence of $\mathbf{H .} 4$-HBA or vanillic acid $(3.3 \mu \mathrm{mol}, 6.88$ $\mathrm{mM}$ final concentration, 0.05 eq.) and laccase $\left(4.4 \mathrm{U}, 9 \mathrm{U} \mathrm{ml}^{-1}\right)$ were dissolved in an ammonium acetate/acetic acid buffer ( $480 \mu \mathrm{l}, 0.1 \mathrm{M}, \mathrm{pH}$ 5). PES model compound $\mathbf{H}$ ( $24 \mathrm{mg}, 66 \mu \mathrm{mol}, 1$ eq.) was added and the mixture was shaken at $40{ }^{\circ} \mathrm{C}$ for $24 \mathrm{~h}$. Alternatively, 4-HBA $(0.46 \mathrm{mg}, 3.3 \mu \mathrm{mol}, 6.6 \mathrm{mM}$ final concentration, 0.1 eq.), TEMPO ( $13 \mu \mathrm{l}$ of a $10 \mathrm{mg}$ per $25 \mathrm{ml}$ ( $1 \%$ methanol in buffer) stock, 33 nmol, 0.01 eq.) and laccase (2.3 $\left.\mathrm{U}, 4.5 \mathrm{U} \mathrm{ml}^{-1}\right)$ were dissolved in an ammonium acetate/acetic acid buffer (500 $\mu \mathrm{l}, 0.1 \mathrm{M}, \mathrm{pH}$ 5). PES model compound $\mathbf{H}$ ( $12 \mathrm{mg}, 33 \mu \mathrm{mol}, 1$ eq.) was added and the mixture was shaken at it for $24 \mathrm{~h}$.

Work-up: the reaction mixture was diluted with methanol and laccase was subsequently removed using a $9 \mathrm{kDa}$ protein concentrator by employing centrifugal force (Hermle Z 206A). The filtrate was thereafter analysed by LC-MS (LC-method LC4).

Laccase-mediated oligomerisation of 3-F-4-HBA in the presence of resin-bound 4-HBA. 3Fluoro-4-hydroxybenzoic acid (3-F-4-HBA, $1.03 \mathrm{mg}, 6.6 \mu \mathrm{mol}, 13.2 \mathrm{mM}$ final concentration, $1.1 \mathrm{eq}$.) and laccase $\left(0.13 \mathrm{U}, 0.26 \mathrm{U} \mathrm{ml}^{-1}\right)$ were dissolved in an aqueous ammonium acetate/acetic acid buffer ( $0.5 \mathrm{ml}, 0.02 \mathrm{M}, \mathrm{pH}$ 5). After addition of resin-bound 4-HBA (10 mg, "6.2 $\mu$ mol" 4-HBA, "1 eq." 4-HBA) the mixture was agitated at it for $2 \mathrm{~h}$. Washing of the modified resin was thereafter conducted with water and methanol consecutively (2x). Cleavage from the resin was achieved in a 1:1 mixture of trifluoroacetic acid and dichloromethane. After evaporation of all volatiles, the cleaved material was analysed by LC-MS (LC-method LC3). For a proper comparison, 3-F-4-HBA and 4-HBA were also oligomerised separately under the same conditions, but without the presence of resin-bound 4-HBA. The resin washing procedure was mimicked by adding methanol $(1 \mathrm{ml})$ to the reaction mixture, followed by evaporation of water and methanol. Cleavage was thereafter simulated by addition of a 1:1 mixture of trifluoroacetic acid and dichloromethane $(2 \mathrm{ml})$ and evaporation of all volatiles.

Laccase-mediated oligomerisation in the presence of R-mPES${ }_{3}$. Either of the phenolics 4-HBA, vanillic acid or syringic acid $(0.15 \mathrm{mmol}, 30.0 \mathrm{mM}$ final concentration, 6 eq.) was dissolved in $0.2 \mathrm{ml}$, $0.5 \mathrm{ml}$ or $1 \mathrm{ml}$ methanol, respectively. This phenol stock and laccase $\left(5 \mathrm{U}, 1 \mathrm{U} \mathrm{ml}^{-1}\right)$ were thereafter dissolved in an aqueous ammonium acetate/acetic acid buffer $(5 \mathrm{ml}, 0.02 \mathrm{M}, \mathrm{pH} 5)$. After addition of R-mPES 3 ( 54 mg, “0.025 mmol" mPES 3 , "1 eq." mPES ${ }_{3}$ ) the mixture was gently shaken at $40{ }^{\circ} \mathrm{C}$ for 60 h. Washing of the modified resin was conducted with dichloromethane, followed by acetone and finally water (3x). Cleavage from the resin was achieved using a 1:1 mixture of trifluoroacetic acid and dichloromethane. After evaporation of all volatiles, the cleaved material was analysed by LC-MS (LCmethod LC2). 
Laccase-mediated oligomerisation in the presence of R-mPES $10.4-\mathrm{HBA}$ (40 mg, $0.30 \mathrm{mmol}$, $30.0 \mathrm{mM}$ final concentration, 6 eq.) and laccase (32 U, $3 \mathrm{U} \mathrm{ml}^{-1}$ ) were dissolved in an aqueous ammonium acetate/acetic acid buffer (10 ml, $0.02 \mathrm{M}, \mathrm{pH}$ 5). After addition of R-mPES 10 (109 mg, "0.05 mmol" mPES 10 , "1 eq." mPES 10 ) the mixture was gently shaken at $40{ }^{\circ} \mathrm{C}$ for $24 \mathrm{~h}$. Washing of the modified resin was achieved with dichloromethane, followed by acetone and finally water (3x). Cleavage from the resin was achieved in a 1:1 mixture of trifluoroacetic acid and dichloromethane. After evaporation of all volatiles, the cleaved material was analysed by LC-MS (LC-method LC2).

\section{Laccase-mediated conversion of charged phenolics in the presence of a PES membrane or sheet}

The positively or negatively charged phenolic ( 28.8 or $57.6 \mathrm{mM}, 1$ eq.), laccase ( 1 or $4 \mathrm{U} \mathrm{ml}^{-1}$ ) and, in those cases where either of the phenols B, C, D or $\mathbf{F}$ was used, TEMPO (0.02 eq.) were dissolved in an aqueous sodium acetate/acetic acid buffer (0.1 M, pH 5). A cleaned PES membrane or sheet was placed in the reaction medium and agitated at it or $40^{\circ} \mathrm{C}$ for $24 \mathrm{~h}$. Alternatively, a $50 \mu \mathrm{l}$ aliquot of the reaction mixture was placed on a cleaned PES sheet which was allowed to stand for $24 \mathrm{~h}$ in the vicinity of a dish filled with water (at the start, this set-up was covered to minimise evaporation). Cleaning of both membranes and sheets was conducted by rinsing with water. Some modified PES sheets were gently wiped with a soft cotton swab for additional cleaning.

\section{Laccase-mediated oligomerisation of 4-HBA in the presence of a PES membrane}

4-HBA (2.0 mg, $0.06 \mathrm{mmol}, 28.8 \mathrm{mM}$ ) and laccase (4 mg, $8 \mathrm{U}, 4 \mathrm{U} \mathrm{ml}^{-1}$ ) were dissolved in an aqueous sodium acetate/acetic acid buffer $(0.1 \mathrm{M}, \mathrm{pH} 5)$ in a petri dish. A rinsed membrane was placed in the reaction medium and agitated at $40^{\circ} \mathrm{C}$ for $4 \mathrm{~h}$. The membrane was then flushed with lukewarm tap water for $2 \mathrm{~min}$ or $5 \mathrm{~min}$ on one side. Additional washing was thereafter either conducted by agitating the membrane in water or in an aqueous solution of TWEEN ${ }^{\circledR}(0.1 \mathrm{wt} \%)$ at 40 ${ }^{\circ} \mathrm{C}$ for $72 \mathrm{~h}$.

\section{Separation and analysis}

LC-MS. For liquid chromatography-mass spectrometry (LC-MS) analysis a MS pump (Thermo Finnigan Surveyor) was coupled to a photodiode array detector (Thermo Finnigan Surveyor PDA) and a mass spectrometer (Finnigan LXQ). Measurements were conducted in negative ionisation mode. Separation was achieved on an Alltima HP C18 column ( $2.1 \mathrm{~mm} \times 100 \mathrm{~mm}, 3 \mu \mathrm{m}$; Grace) at room temperature at a flow rate of $0.2 \mathrm{ml} \mathrm{min}{ }^{-1}$. The mobile phases consisted of $0.1 \%$ formic acid in water (mobile phase A) and in acetonitrile (mobile phase B).

LC1: Separation was achieved through isocratic elution with $15 \%$ B for 5.0 min followed by isocratic elution with $20 \% \mathrm{~B}$ for $12.0 \mathrm{~min}$, after which the column was flushed through isocratic elution with $100 \%$ B for 13.0 min. The column was re-equilibrated by employing $15 \%$ B for 15.0 min.

LC2: Separation was achieved through elution with a linear gradient starting with $20 \%$ B and ending with $100 \% \mathrm{~B}$ after $30.0 \mathrm{~min}$, after which the column was flushed through isocratic elution with $100 \%$ B for 15.0 min. The column was re-equilibrated by employing $20 \%$ B for $15.0 \mathrm{~min}$.

LC3: Separation was achieved through elution with a linear gradient starting with $5 \%$ B and ending with $95 \%$ B after 20.0 min, after which the column was flushed through isocratic elution with 95\% B for $20.0 \mathrm{~min}$. The column was re-equilibrated by employing $5 \%$ B for $12.0 \mathrm{~min}$.

LC4: Separation was achieved through elution with a linear gradient starting with $5 \%$ B and ending with $75 \%$ B after 20.0 min, additional separation was thereafter achieved through isocratic elution with $75 \%$ for 5.0 min. Flushing of the column was subsequently achieved through isocratic elution with $95 \%$ B for 15.0 min ( 25.0 min when using highly concentrated material), after which the column was re-equilibrated by employing $5 \%$ B for $12.0 \mathrm{~min}$. 
Preparative HPLC. Preparative HPLC-separations were performed on an Alltima C18 column $(22 \mathrm{~mm}$ $\times 250 \mathrm{~mm}, 5 \mu \mathrm{m}$; Grace). A LC-8A Shimadzu LC-pump was connected to a SPD-M10AVP Shimadzu diode array detector. Separation was achieved at room temperature through elution with $3 \%$ methanol

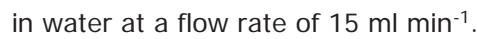

FTIR. FTIR spectra were measured on a diamond crystal (Platinum ATR, Bruker) integrated in a Bruker Tensor 27 spectrophotometer system controlled through OPUS software (version 7.2.139.1294). All spectra were recorded with at least 16 scans, using ambient air as background.

XPS. X-ray photoelectron spectroscopy (XPS) core electron binding energies were measured on a JPS9200 photoelectron spectrometer (J EOL). Surfaces were irradiated at one or more spots with an Al Ka source emitting $1486.7 \mathrm{eV}$ photons while charge compensation was being applied with an accelerating voltage of $2.8 \mathrm{eV}$ and a filament current of $4.80 \mathrm{~A}$ to compensate for charging of the carbon-based polymer surfaces. Spectra were analysed using CasaXPS version 2.3.16 PR 1.6. The C 1s peak emission was calibrated to a binding energy of $284.5 \mathrm{eV}$ when measuring on PES, and C 1s narrow scans were deconvoluted into their component peaks using Gaussian-Lorentzian sum functions having $30 \%-70 \%$ Gaussian-Lorentzian character.

SWCA measurement. SWCA measurements were performed on a Krüss DSA 100 drop shape analyser. SWCA's were assessed for $3 \mu \mathrm{l}$ water droplets, where drop shape was fitted according to Tangent-2 fitting parameters (Krüss DSA software version 1.90.0.14) by fitting at least 10× for each droplet. Reported values are an average of SWCA's measured over multiple droplets on a surface.

\section{Acknowledgements}

The authors would like to thank Judith Firet, Adrie H. Westphal and Mahboubeh Tasviri for their assistance in experimental work. This project was funded by the NanoNextNL project 04A.01 "Biomimetic selective layers".

\section{References}

[1] a) R. Zhang, Y. Liu, M. He, Y. Su, X. Zhao, M. Elimelech, Z. Jiang, Chem. Soc. Rev. 2016, 45, 5888-5924; b) P. Le-Clech, V. Chen, T. A. G. Fane, J. Membr. Sci. 2006, 284, 17-53; c) L. F. Greenlee, D. F. Lawler, B. D. Freeman, B. Marrot, P. Moulin, Water Res. 2009, 43, 2317-2348.

[2] H. Flemming, Appl. Microbiol. Biotechnol. 2002, 59, 629-640.

[3] V. Moghimifar, A. Raisi, A. Aroujalian, J. Membr. Sci. 2014, 461, 69-80.

[4] X. Li, T. Cai, T. Chung, Environ. Sci. Technol. 2014, 48, 9898-9907.

[5] E. Celik, H. Park, H. Choi, H. Choi, Water Res. 2011, 45, 274-282.

[6] N. Nady, M. C. R. Franssen, H. Zuilhof, M. S. Mohyeldin, R. Boom, K. Schroën, Desalination 2011, 275, 1-9.

[7] S. Schmidt, C. Scherkus, J. Muschiol, U. Menyes, T. Winkler, W. Hummel, H. Gröger, A. Liese, H. G. Herz, U. T. Bornscheuer, Angew. Chem. Int. Ed. 2015, 54, 2784-2787.

[8] a) Y. Jiang, A. J. J. Woortman, G. O. R. Alberda van Ekenstein, D. M. Petrović, K. Loos, Biomacromolecules 2014, 15, 2482-2493; b) P. Steunenberg, M. Uiterweerd, M. Sijm, E. L. Scott, H. Zuilhof, J. P. M. Sanders, M. C. R. Franssen, Curr. Org. Chem. 2013, 17, 682-690.

[9] N. Nady, K. Schroën, M. C. R. Franssen, B. van Lagen, S. Murali, R. M. Boom, M. S. Mohyeldin, H. Zuilhof, ACS Appl. Mater. Interfaces 2011, 3, 801-810.

[10] a) N. Nady, K. Schroën, M. C. R. Franssen, R. Fokkink, M. S. Mohyeldin, H. Zuilhof, R. M. Boom, J. Colloid Interface Sci. 2012, 378, 191-200; b) S. van der Veen, N. Nady, M. C. R. Franssen, H. Zuilhof, R. M. Boom, T. Abee, K. Schroën, J. Appl. Polym. Sci. 2015, 132, 41576.

[11] V. Madhavi, S. S. Lele, Bioresources 2009, 4, 1694-1717. 
[12] S. Slagman, J. Escorihuela, H. Zuilhof, M. C. R. Franssen, RSC Adv. 2016, 6, 99367-99375.

[13] a) X. Li, S. De Feyter, D. Chen, S. Aldea, P. Vandezande, F. Du Prez, I. F. J. Vankelecom, Chem. Mater. 2008, 20, 3876-3883; b) R. Malaisamy, M. L. Bruening, Langmuir 2005, 21, 10587-10592; c) J. Kochan, T. Wintgens, J. E. Wong, T. Melin, Desalination 2010, 250, 10081010.

[14] a) D. Saeki, M. Imanishi, Y. Ohmukai, T. Maruyama, H. Matsuyama, J. Membr. Sci. 2013, 447, 128-133; b) Q. Chen, P. Yu, W. Huang, S. Yu, M. Liu, C. Gao, J. Membr. Sci. 2015, 492, $312-$ 321.

[15] a) R. Hadj Lajimi, E. Ferjani, M. S. Roudesli, A. Deratani, Desalination 2011, 266, 78-86; b) L. Y. Ng, A. W. Mohammad, C. Y. Ng, C. P. Leo, R. Rohani, Desalination 2014, 351, 19-26.

[16] a) G. Zhang, W. Gu, S. Ji, Z. Liu, Y. Peng, Z. Wang, J. Membr. Sci. 2006, 280, 727-733; b) U. K. Aravind, J. Mathew, C. T. Aravindakumar, J. Membr. Sci. 2007, 299, 146-155.

[17] H. Wu, X. Li, C. Zhao, X. Shen, Z. Jiang, X. Wang, Ind. Eng. Chem. Res. 2013, 52, 5772-5780.

[18] Z. J ia, D. Shen, W. Xu, Carbohydr. Res. 2001, 333, 1-6.

[19] T. Kudanga, E. Nugroho Prasetyo, J. Sipilä, P. Nousiainen, P. Widsten, A. Kandelbauer, G. S. Nyanhongo, G. M. Guebitz, Eng. Life Sci. 2008, 8, 297-302.

[20] L. Servillo, A. Giovane, N. D'Onofrio, R. Casale, D. Cautela, G. Ferrari, M. L. Balestrieri, D. Castaldo, J. Agric. Food Chem. 2014, 62, 2679-2684.

[21] a) D. Skalamera, C. Bohne, S. Landgraf, N. Basaric, J. Org. Chem. 2015, 80, 10817-10828; b) J. H. Hodgkin, Aust. J. Chem. 1984, 37, 2371-2378.

[22] R. Ikeda, J. Sugihara, H. Uyama, S. Kobayashi, Polym. Int. 1998, 47, 295-301.

[23] F. Xu, Appl. Biochem. Biotechnol. 1996, 59, 221-230.

[24] T. Miyano, T. Matsuura, S. Sourirajan, Chem. Eng. Commun. 1993, 119, 23-39.

[25] a) T. Kudanga, E. Nugroho Prasetyo, J. Sipilä, G. S. Nyanhongo, G. M. Guebitz, Enzyme Microb. Technol. 2010, 46, 272-280; b) S. Slagman, H. Zuilhof, M. C. R. Franssen, ChemBioChem 2018, 19, 288-311.

[26] J. Bollag, S. Liu, R. D. Minard, Soil Biol. Biochem 1982, 14, 157-163.

[27] F. García-Martín, M. Quintanar-Audelo, Y. García-Ramos, L. J. Cruz, C. Gravel, R. Furic, S. Côté, J. Tulla-Puche, F. Albericio, J. Comb. Chem. 2006, 8, 213-220.

[28] M. Lund, A. Ragauskas, Appl. Microbiol. Biotechnol. 2001, 55, 699-703.

[29] P. Iqbal, K. Critchley, J. Bowen, D. Attwood, D. Tunnicliffe, S. D. Evans, J. A. Preece, J. Mater. Chem. 2007, 17, 5097-5110.

[30] A. Lesac, S. Narančić, M. Šepelj, D. W. Bruce, V. Šunjić, Tetrahedron: Asymmetry 2003, 14, 2731-2737. 


\section{Supporting information corresponding to Chapter 3}

Figure S1. XPS wide scan of PES membrane after incubation with $A(28.8 \mathrm{mM})$ and laccase $\left(4 \mathrm{U} \mathrm{ml}^{-1}\right)$ at room temperature.

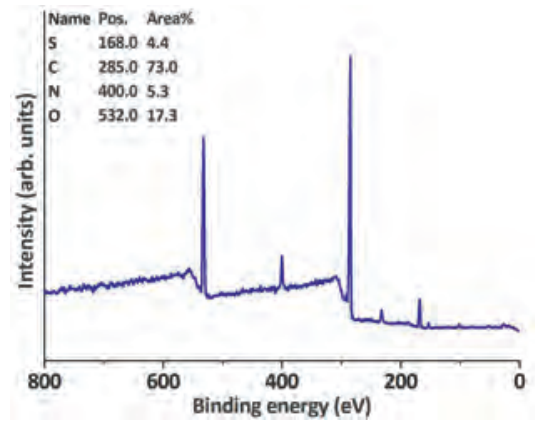

Corresponds to Table 1 , entry 2.

Figure S3. XPS wide scan of PES sheet after incubation with $B(57.6 \mathrm{mM})$ and laccase (4 $\mathrm{U} \mathrm{ml}^{-1}$ ) at $40^{\circ} \mathrm{C}$.

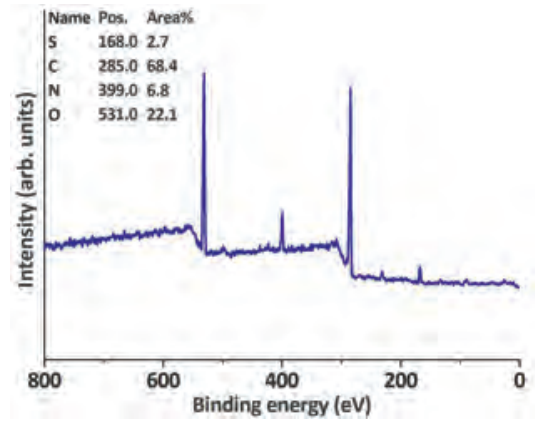

Corresponds to Table 1 , entry 6.
Figure S2. XPS N 1s narrow scan of PES sheet after incubation with $B(28.8 \mathrm{mM})$ and laccase $\left(4 \mathrm{U} \mathrm{ml}^{-1}\right)$ at room temperature.

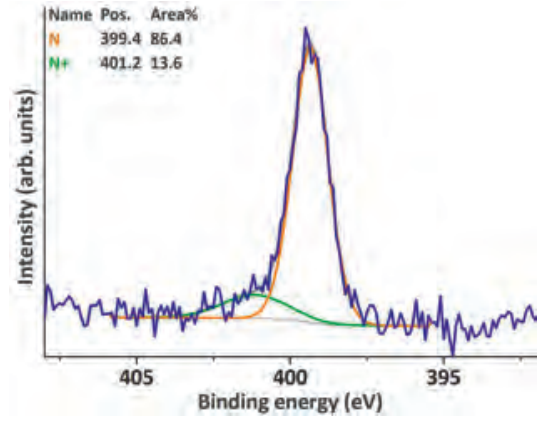

Corresponds to Table 1 , entry 3.

Figure S4. XPS $\mathbf{N} 1 s$ narrow scan of PES sheet after incubation with $B(57.6 \mathrm{mM})$ and laccase $\left(4 \mathrm{U} \mathrm{ml}^{-1}\right)$ at $40{ }^{\circ} \mathrm{C}$ and subsequent wiping.

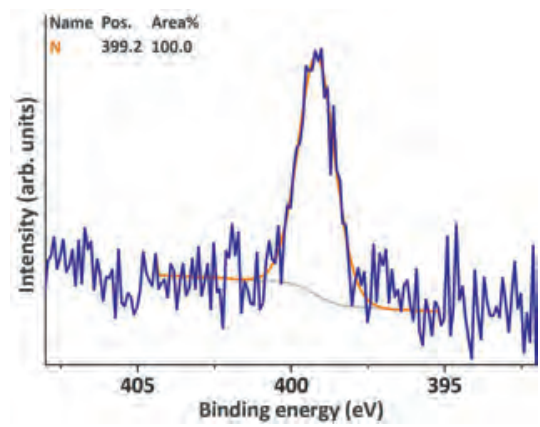

Corresponds to Table 1, entry 7. 
Figure S5. XPS wide scan of PES sheet after incubation with $B(57.6 \mathrm{mM})$ and laccase (4 $\mathrm{U} \mathrm{ml}^{-1}$ ) at $40^{\circ} \mathrm{C}$ and subsequent wiping.

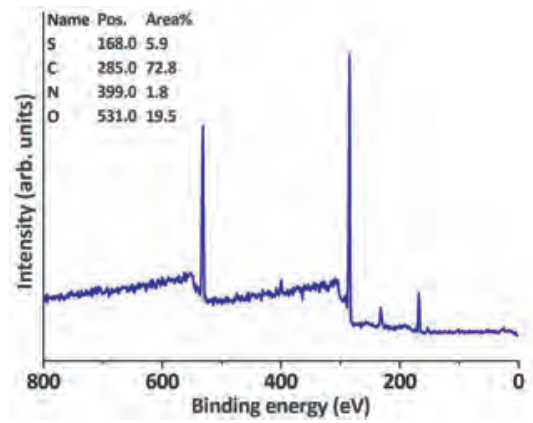

Corresponds to Table 1, entry 7.

Figure S7. XPS N 1s narrow scan of PES sheet after incubation with $\mathrm{C}(28.8 \mathrm{mM})$ and laccase $\left(4 \mathrm{U} \mathrm{ml}^{-1}\right)$ at $40{ }^{\circ} \mathrm{C}$.

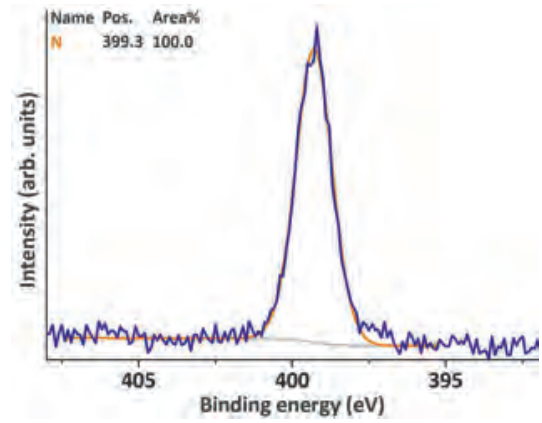

Corresponds to Table 1, entry 10.

Figure S9. XPS wide scan of PES sheet after incubation with $D(28.8 \mathrm{mM})$ and laccase (4 $\mathrm{U} \mathrm{ml}^{-1}$ ) at room temperature.

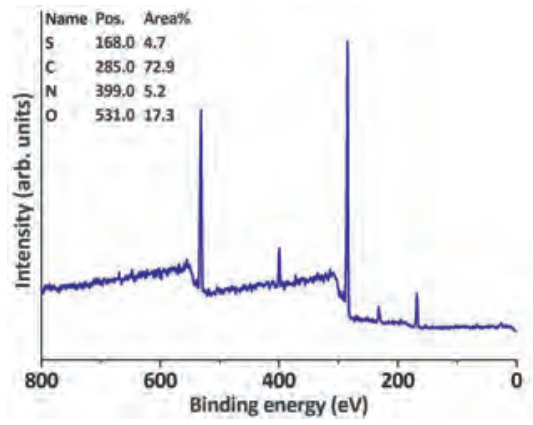

Corresponds to Table 1, entry 12.
Figure S6. XPS N 1s narrow scan of PES sheet after incubation with C (28.8 $\mathrm{mM})$ and laccase $\left(4 \mathrm{U} \mathrm{ml}^{-1}\right)$ at room temperature.

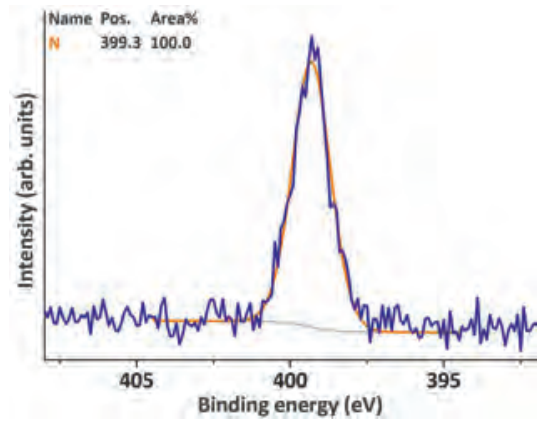

Corresponds to Table 1, entry 8.

Figure S8. XPS $\mathbf{N}$ 1s narrow scan of PES sheet after incubation with $D(28.8 \mathrm{mM})$ and laccase $\left(4 \mathrm{U} \mathrm{ml}^{-1}\right)$ at room temperature.

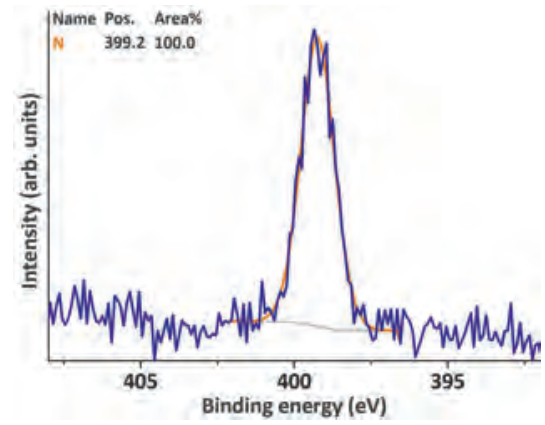

Corresponds to Table 1, entry 12.

Figure S10. XPS N 1s narrow scan of PES sheet after incubation with $F(28.8 \mathrm{mM})$ and laccase $\left(4 \mathrm{U} \mathrm{ml}^{-1}\right)$ at room temperature.

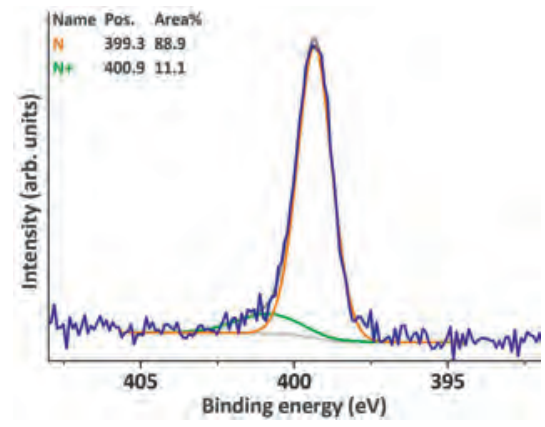

Corresponds to Table 1, entry 14. 
Figure S11. XPS N 1s narrow scan of PES sheet after incubation with laccase $\left(4 \mathrm{U} \mathrm{ml}^{-1}\right)$ at room temperature.

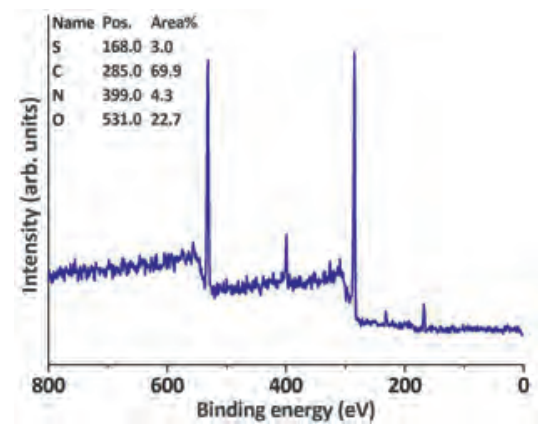

Corresponds to Table 1, entry 16.

Figure S13. XPS wide scan of PES sheet after incubation with $A(28.8 \mathrm{mM})$ and purified laccase $\left(4 \mathrm{U} \mathrm{ml}^{-1}\right)$ at room temperature.

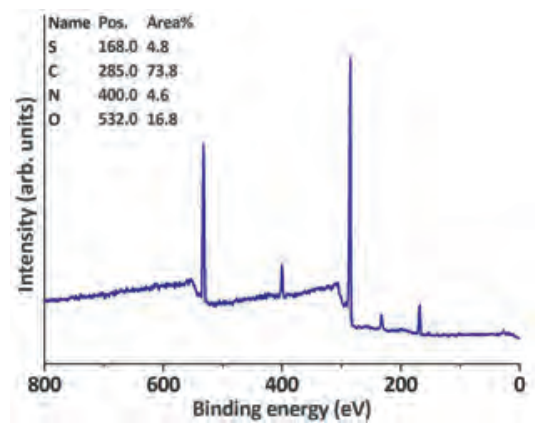

Figure S15. XPS N 1s narrow scan of F3 drop-casted on an etched silicon wafer.

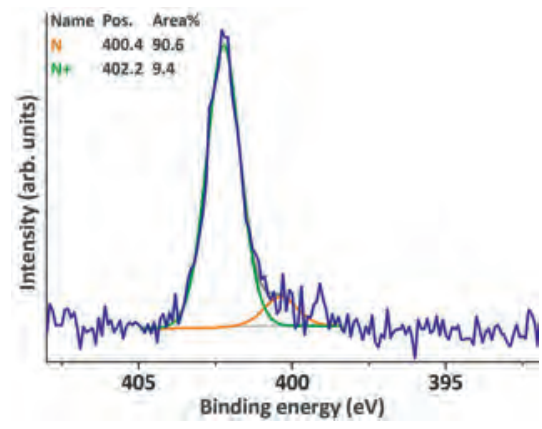

Figure S12. XPS $N$ 1s narrow scan of PES sheet after incubation with $A(28.8 \mathrm{mM})$ and purified laccase $\left(4 \mathrm{U} \mathrm{ml}^{-1}\right)$ at room temperature.

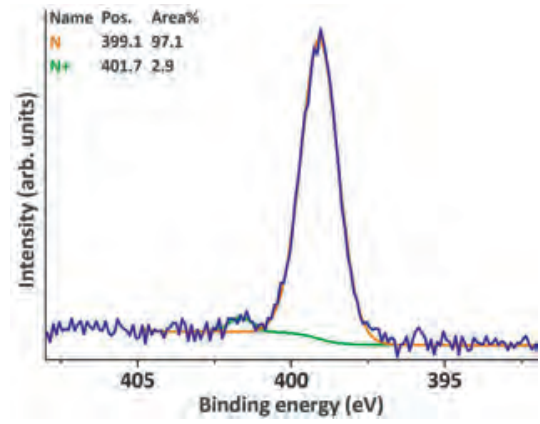

Figure S14. XPS wide scan of blank (nonmodified) PES sheet.

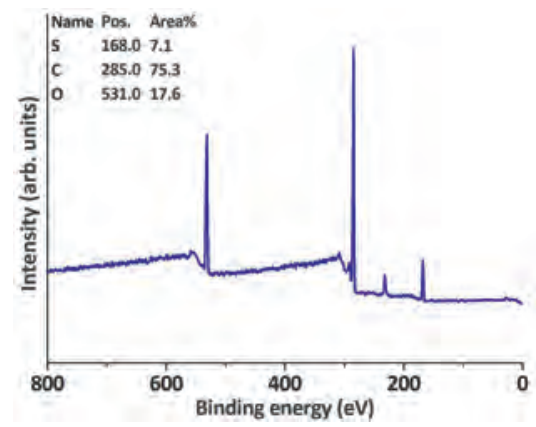

Corresponds to Table 1, entry 19. 
Figure S16. LC-MS analysis of the reaction medium after incubation of 4-HBA, laccase and $\mathrm{H}$.

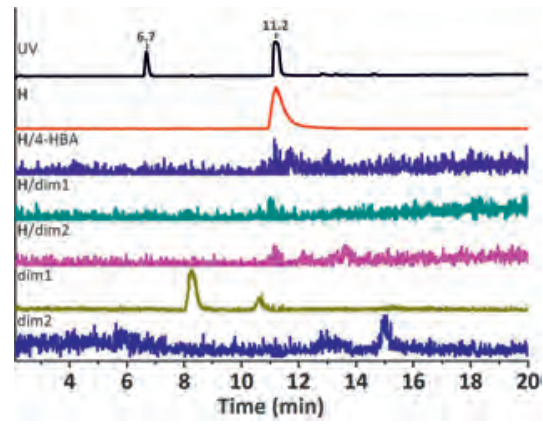

UV and extracted ion chromatograms of ions whose $\mathrm{m} / \mathrm{z}$ ([M-H] $\left.]^{-} \pm 0.50 \mathrm{Da}\right)$ corresponds to that of $\mathbf{H}, \operatorname{dim} 1 / \operatorname{dim} 2$ or their conjug. after incub. of 4-HBA, lac. and $\mathbf{H}$.

Figure S18. LC-MS analysis of the highly concentrated reaction medium after incubation of 4-HBA, laccase and $H$.

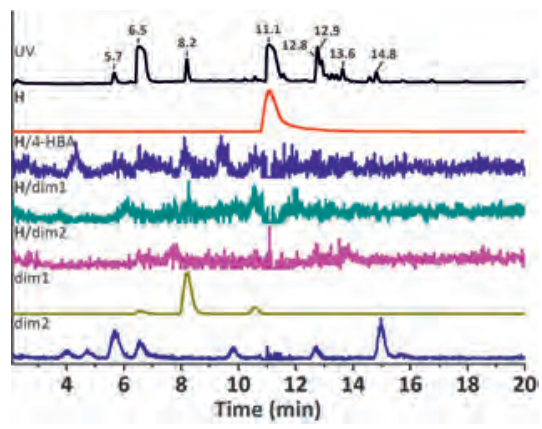

UV and extracted ion chromatograms of ions whose $\mathrm{m} / \mathrm{z}$ $\left([\mathrm{M}-\mathrm{H}]^{-} \pm 0.50 \mathrm{Da}\right)$ corresponds to that of $\mathbf{H}, \operatorname{diml} / \mathrm{dim} 2$ or their conjug. after incub. of 4-HBA, lac. and $\mathbf{H}$.

Figure S20. Mass spectrum corresponding to peak at $6.5 \mathrm{~min}$ in SI Figure S18.

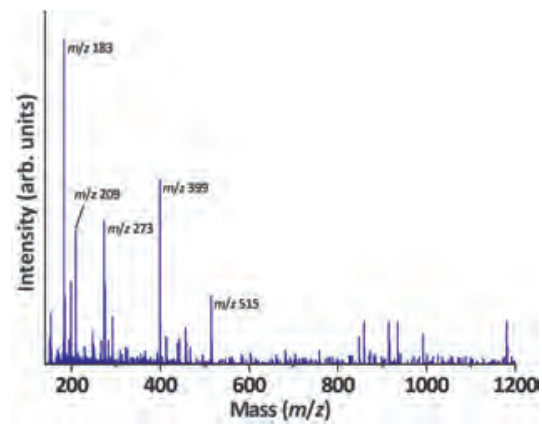

Figure S17. LC-MS analysis of the reaction medium after incubation of 4-HBA, laccase, TEMPO and $\mathrm{H}$.

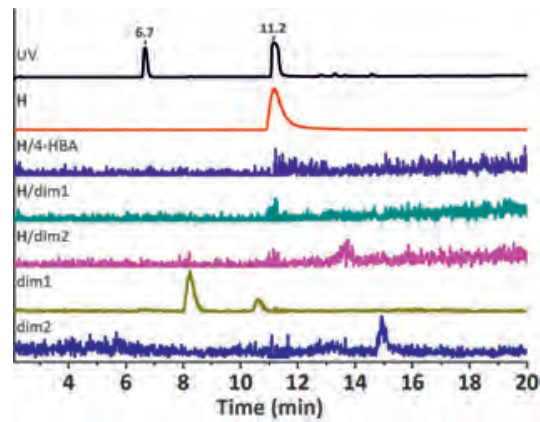

UV and extracted ion chromatograms of ions whose $m / z$ $\left([\mathrm{M}-\mathrm{H}]^{-} \pm 0.50 \mathrm{Da}\right)$ corresponds to that of $\mathbf{H}$, diml/dim2 or their conjug. after incub. of 4-HBA, lac., TEMPO and $\mathbf{H}$.

Figure S19. Mass spectrum corresponding to peak at $5.7 \mathrm{~min}$ in SI Figure S18.

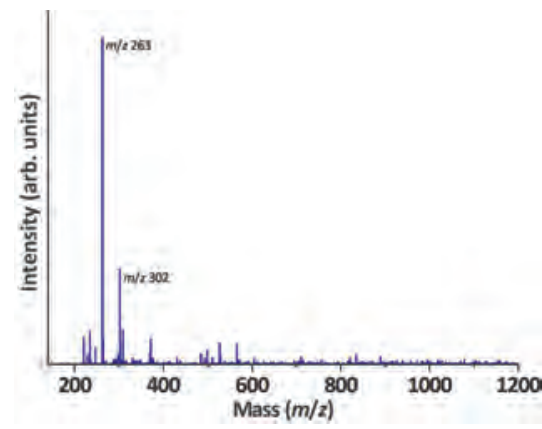

Figure S21. Mass spectrum corresponding to peak at $8.2 \mathrm{~min}$ in SI Figure S18.

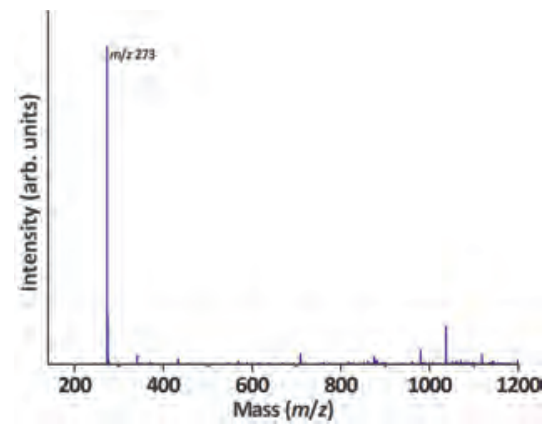


Figure S22. Mass spectrum corresponding to peak at $11.1 \mathrm{~min}$ in SI Figure S18.

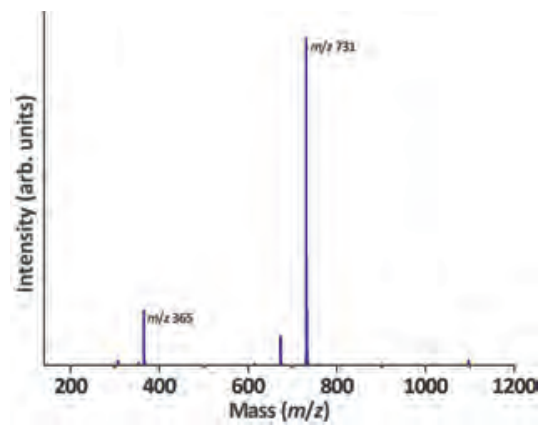

Figure S24. Mass spectrum corresponding to peak at $\mathbf{1 2 . 9} \mathbf{~ m i n ~ i n ~ S I ~ F i g u r e ~ S 1 8 . ~}$

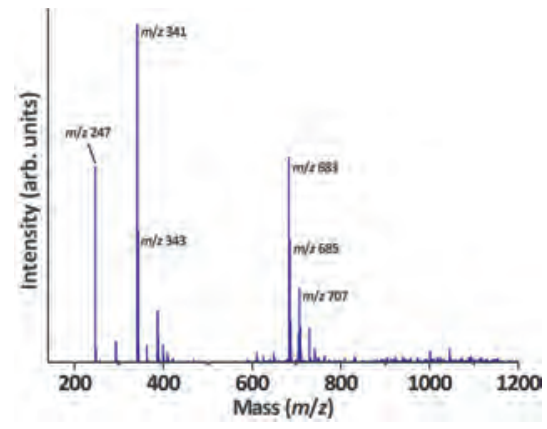

Figure S26. Mass spectrum corresponding to peak at $14.8 \mathrm{~min}$ in SI Figure S18.

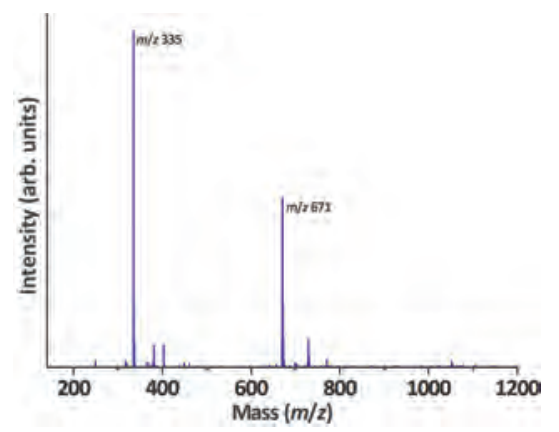

Figure S23. Mass spectrum corresponding to peak at $\mathbf{1 2 . 8} \mathbf{~ m i n ~ i n ~ S I ~ F i g u r e ~ S 1 8 . ~}$

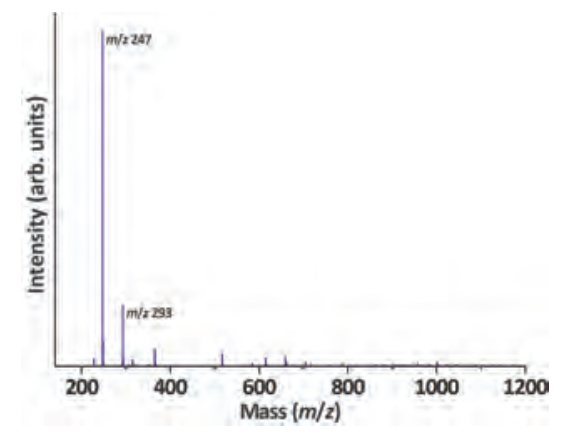

Figure S25. Mass spectrum corresponding to peak at $\mathbf{1 3 . 6} \mathbf{~ m i n}$ in SI Figure S18.

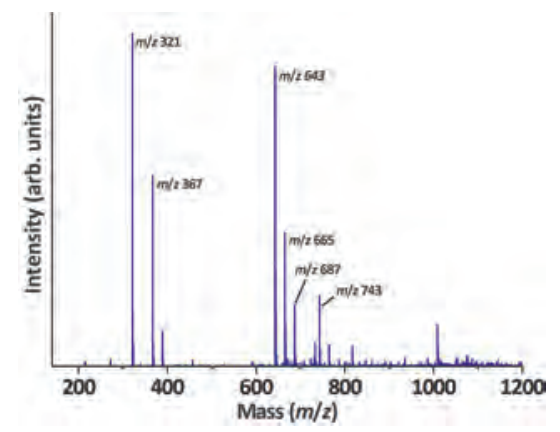


Figure S27. LC-MS analysis of the reaction medium after incubation of vanillic acid (VA), laccase and $H$.

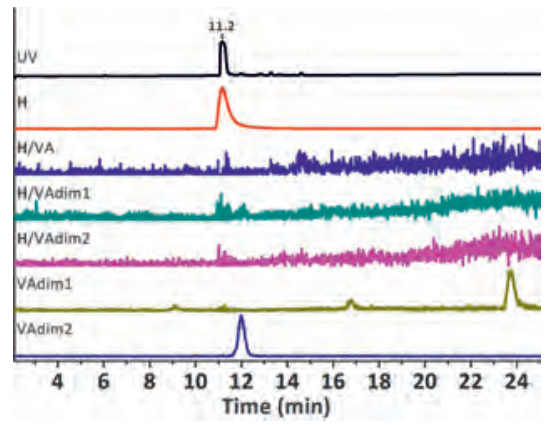

UV trace and extracted ion chromatograms of ions whose $\mathrm{m} / \mathrm{z}\left([\mathrm{M}-\mathrm{H}]^{-} \pm 0.50 \mathrm{Da}\right)$ corresponds to that of $\mathbf{H}$, VAdim1/VAdim2 or their conjugates after incubation of VA, laccase and $\mathbf{H}$.

Figure S29. LC-MS analysis of the material cleaved from R-mPES 3 after its incubation with syringic acid and laccase.

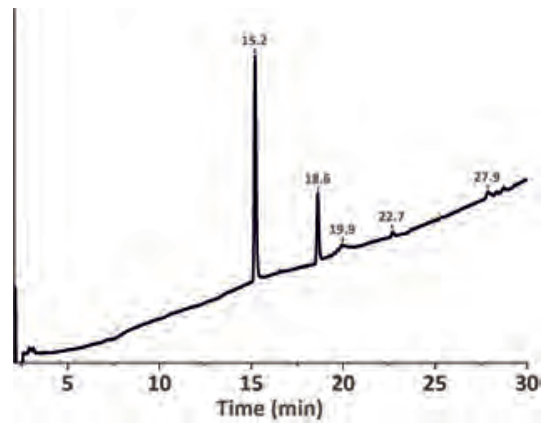

Figure S31. Mass spectrum corresponding to peak at 22.7 min in SI Figure S29.

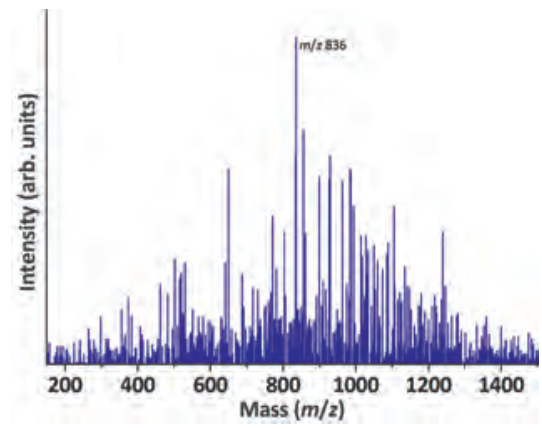

Figure S28. LC-MS analysis of the material cleaved from R-mPES 3 after its incubation with vanillic acid (VA) and laccase.

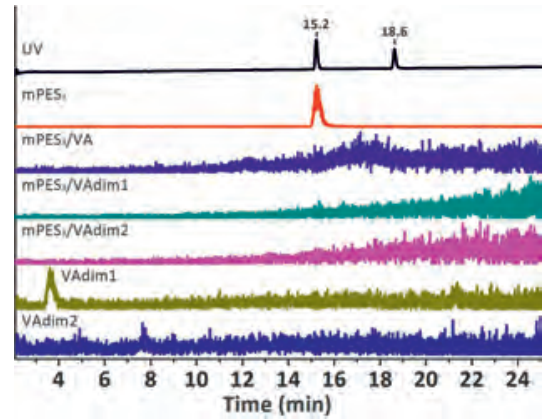

UV trace and extracted ion chromatograms of ions whose $m / z\left([\mathrm{M}-\mathrm{H}]^{-} \pm 0.50 \mathrm{Da}\right)$ corresponds to that of $\mathrm{mPES}_{3}$, VAdiml/VAdim2 or their conjugates after incubation of VA, laccase and R-mPES 3 .

Figure S30. Mass spectrum corresponding to peak at $19.9 \mathrm{~min}$ in SI Figure S29.

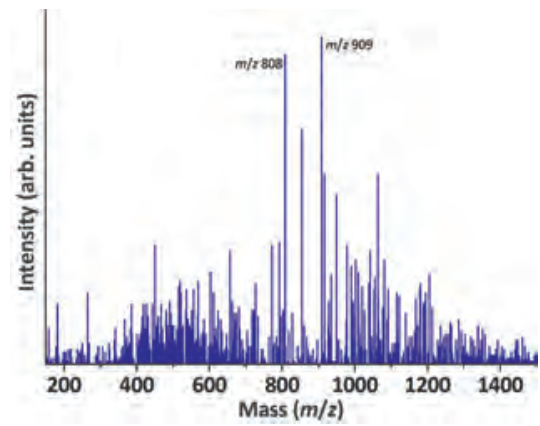

Figure S32. Mass spectrum corresponding to peak at 27.9 min in SI Figure S29.

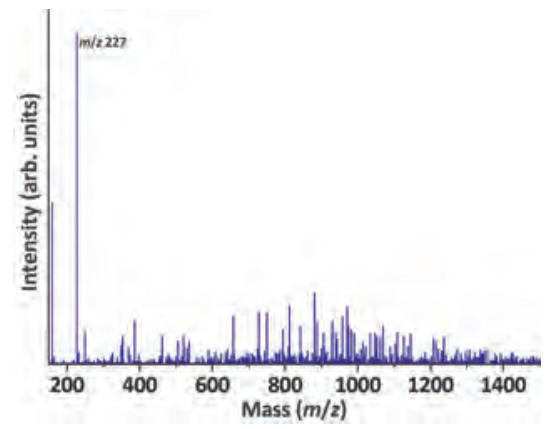


Figure S33. LC-MS analysis of the reaction medium after incubation of 4-HBA, laccase and R-mPES 10 .

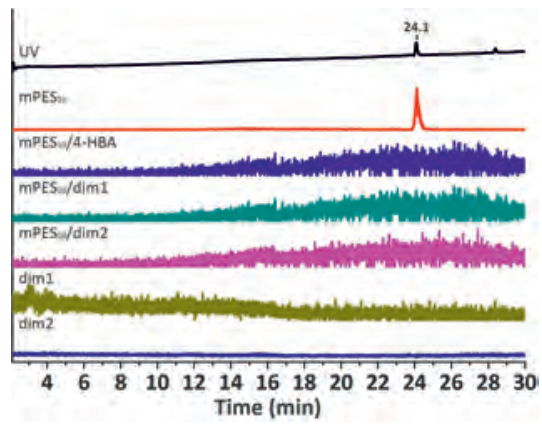

UV trace and extracted ion chromatograms of ions whose $\mathrm{m} / \mathrm{z}\left([\mathrm{M}-\mathrm{H}]^{-} \pm 0.50 \mathrm{Da}\right)$ corresponds to that of $\mathrm{mPES}_{10}$, $\operatorname{dim} 1 / \operatorname{dim} 2$ or their conjugates after incubation of 4-HBA, laccase and R-mPES 10 .

Figure S34. SDS-PAGE gel of purified laccase (Lac) and mass markers (M).

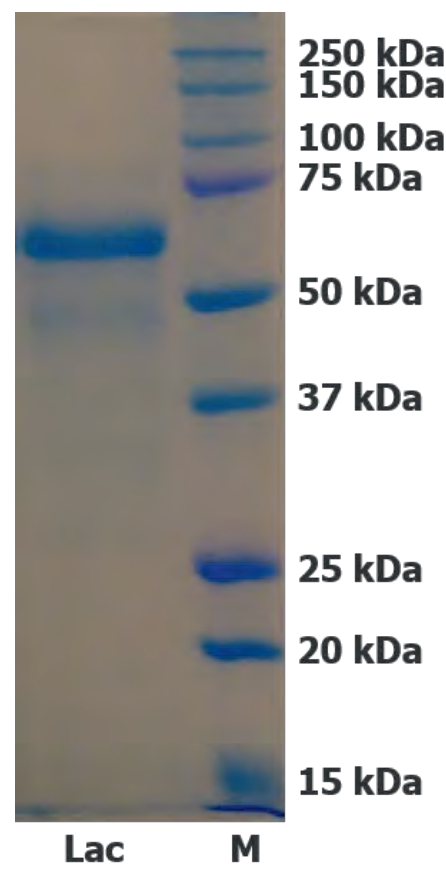




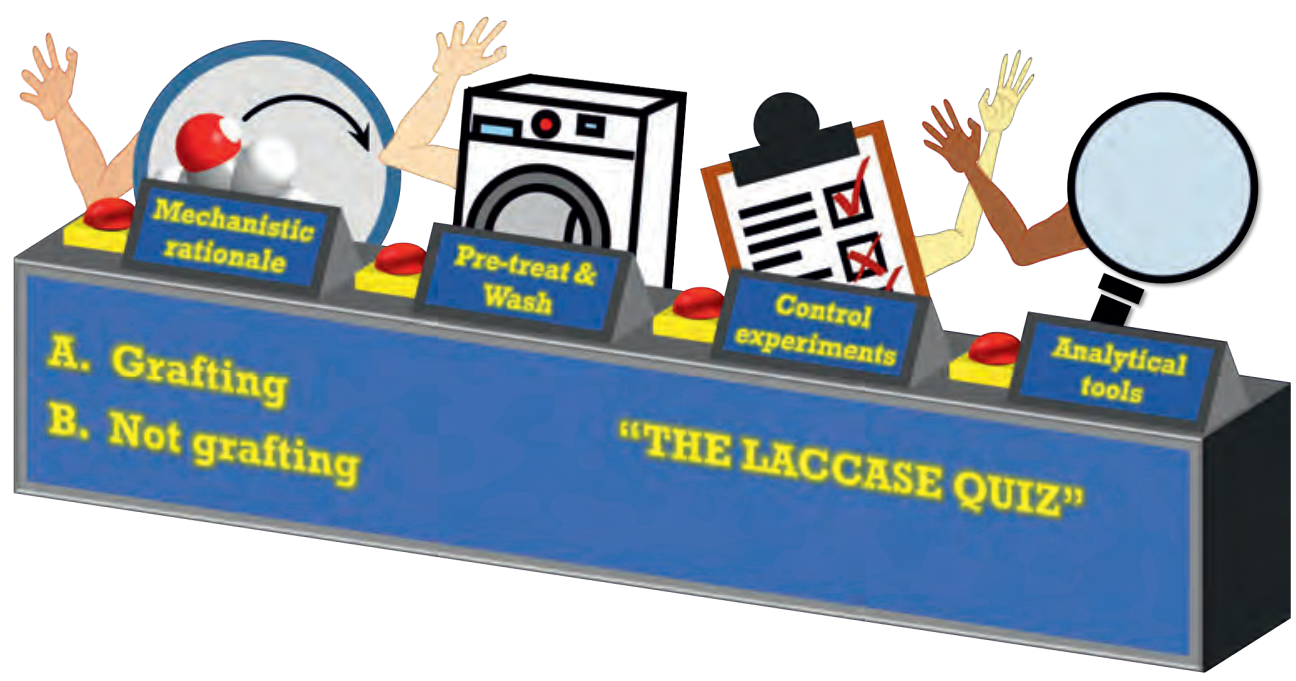




\title{
Chapter 4
}

\section{Laccase-mediated grafting on biopolymers and synthetic polymers: a critical review}

\begin{abstract}
Laccase-mediated grafting on lignocelluloses has gained considerable attention as an environmentally benign strategy for covalently modifying wood, paper and cork. In recent decades this technique has also been employed for modifying fibres with a polysaccharide backbone, such as cellulose or chitosan, to infer colouration, anti-microbial activity or anti-oxidant activity to the material. The scope of this approach has been further widened by researchers who apply mediators or high redox potential laccases and those that modify synthetic polymers and proteins. In all cases the methodology relies on a one- or two-electron oxidation of the surface functional groups or of the graftable molecule in solution. However, similar results can very often be achieved through simple deposition, even after extensive washing. This unintended adsorption of the active substance could have an adverse effect on the durability of the applied coating. Differentiating between actual covalent binding and adsorption is therefore essential but proves to be challenging. This review not only covers excellent research on the topic of laccase-mediated grafting over the last five to ten years, but will also critically compare it side by side to highlight either the lack or presence of compelling evidence for covalent grafting.
\end{abstract}




\section{I ntroduction}

Grafting of (bio)molecules on polymers and surfaces has gained substantial interest in recent years. Grafting concerns covalent bond formation between a macromolecule and a small-molecule. In the context of polymer chemistry, grafting is defined as "a reaction in which one or more species of block are connected to the main chain of a macromolecule as side-chains having constitutional or configurational features that differ from those in the main chain". ${ }^{[1]}$ From a surface chemistry perspective, one needs to expand this definition to composites in which the main chain constitutes a diverse array of materials, ranging from brick ${ }^{[2]}$ and fibreglass ${ }^{[3]}$ to paper ${ }^{[4]}$ and wood. ${ }^{[5]}$ Grafting can be achieved via three different pathways: grafting-to, grafting-from and grafting-through (Scheme 1). ${ }^{[6]}$ Grafting-to is characterised by the attachment of polymer chains bearing a reactive moiety that covalently binds to a compatible reactive group present on the backbone of the composite or macromolecule. Functionalising poly(ethylenimine) with isocyanate-terminated poly(ethylene glycol) to enhance gene delivery systems is an example of a grafting-to approach. ${ }^{[7]}$ In the grafting-from approach, polymerisation of soluble monomers is initiated from the backbone of the composite or macromolecule. Surface-initiated atom transfer radical polymerisation (SI-ATRP) is often used to polymerise zwitterionic monomers via a radical initiator on the surface, so as to confer anti-fouling properties to the surface. ${ }^{[8]}$ Lastly, grafting-through involves the co-polymerisation of bare monomers with monomers bearing the graftable moiety to simultaneously form the graft and the backbone. ${ }^{[9]}$

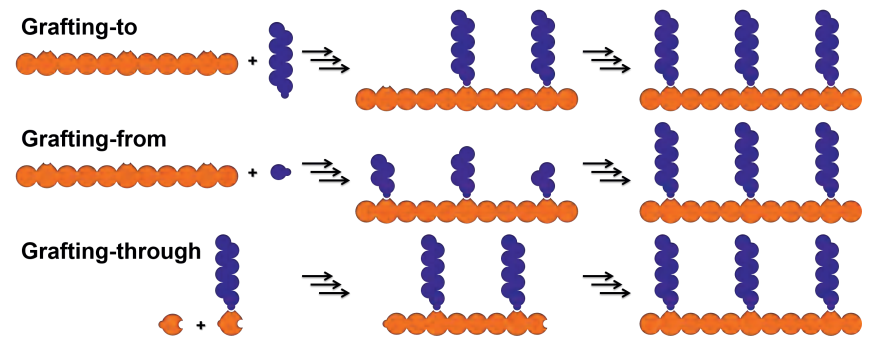

Scheme 1. Three different types of grafting: grafting-to, grafting-from and grafting-through.

Whichever method is chosen, very often (expensive) metal catalysis or the use of harsh conditions is required. In the previously mentioned ATRP process copper $(1)$ complexes are used that, in the presence of oxygen, lead to oxidised metal species, which are detrimental to the turnover of the catalysts. ${ }^{[10]}$ In such cases stringent oxygen-free conditions are essential, which make the grafting procedure more time consuming, more complex and more expensive. Similarly; although new developments allow photografting initiated by visible light, ${ }^{[11]}$ grafting acrylic monomers/polymers often requires high energy UV irradiation. ${ }^{[12]}$ Additionally, the thus formed radicals are susceptible to quenching by oxygen.

In light of the growing world population and the concomitant scarcity of resources, more and more effort is being put forth in circumventing the use of expensive metal catalysts and high energy processes. One way of bypassing these disadvantages is the use of enzymes to initiate grafting. Enzymes operate under mild conditions, are highly tuneable and most often very selective. In the context of grafting, particularly the catalytic use of oxidoreductases has gained considerable attention over the last ten years. ${ }^{[13]}$ Within this class, laccases (EC 1.10.3.2) are of particular interest, since they can oxidise a substrate using molecular oxygen as the oxidant to initiate radical grafting with water as sole by-product, whereas other oxidoreductases, such as horseradish peroxidase (HRP), require hydrogen peroxide as oxidant, which is hazardous at elevated concentrations. ${ }^{[14]}$ The earliest documentation of laccases dates back to the $19^{\text {th }}$ century. In 1883 Yoshida described the isolation of this enzyme from the lacquer tree Rhus vernicifera. ${ }^{[15]}$ More than 100 years later this research is still significant in the production of artificial Urushi lacquer using laccase isolates. ${ }^{[16]}$ Apart from its presence in plants, laccases have also been found in insects ${ }^{[17]}$ and prokaryotes. ${ }^{[18]}$ They are, 
however, most ubiquitous in fungi, particularly in white rot fungi such as Trametes versicolor. ${ }^{[19]}$ The role of laccases in the fungal biome is to assist in the formation of nutrients by degrading lignin to smaller molecules. Laccases act on the omnipresent phenolic moieties in lignin (Scheme 2), however, laccases exhibit a far larger substrate scope than only lignin-derived phenols. Depending on the type of laccase, the scope ranges from catechols to phenols, and even non-phenolic substrates can be converted directly using laccase (although laccases form a family of enzymes, we will typically use the singular, as usually only one type of laccase is used in an experiment). ${ }^{[20]}$ By employing mediators, laccase is able to oxidise molecules that are normally too sterically congested to be oxidised directly, which further expands the scope of this already versatile enzyme. ${ }^{[21]}$

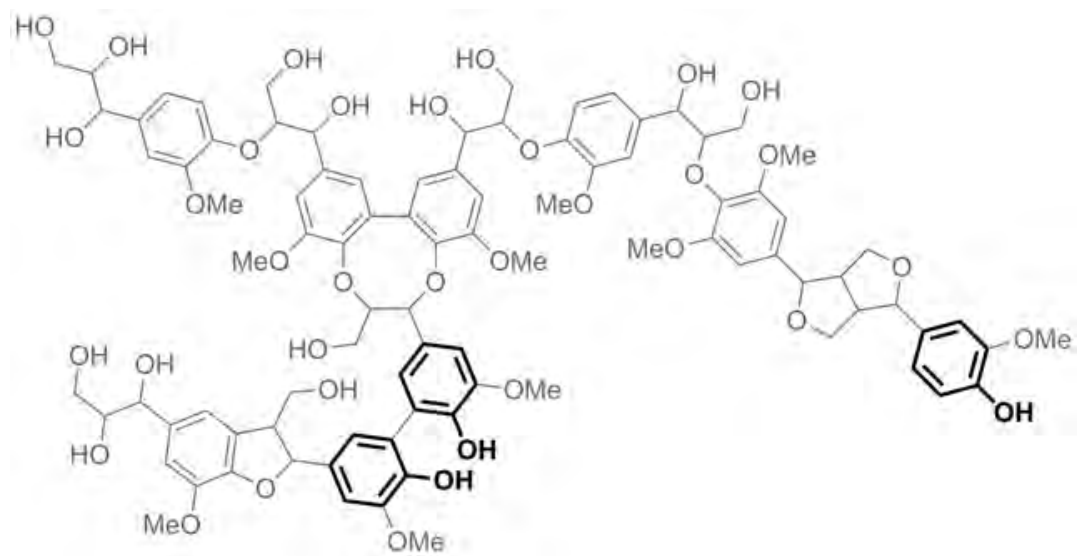

Scheme 2. A small lignin segment in which the phenolic moieties are highlighted.

This wide scope and the ambient conditions under which these enzymes operate are also the reasons for the widespread use of laccase in a variety of applications. Comparable to its native function, laccases are used to degrade lignin in order to obtain pharmaceutically interesting building blocks. ${ }^{[22]}$ They also have an enormous impact in environmental protection through the detoxification of dyes and pharmaceuticals from soil or wastewater. ${ }^{[23]}$ Since laccase-assisted reactions involve radical chemistry, laccases not only aid in breaking molecules down, but also in building them up. For this reason, laccases are used to synthesise small-molecules such as dyestuffs. ${ }^{[20 a, 24]}$ Furthermore, the previously mentioned grafting is a field in which laccase plays an essential role. At the basis of all of these applications is the, among laccases, highly conserved four-atom copper cluster (Scheme 3). ${ }^{[19 b]}$

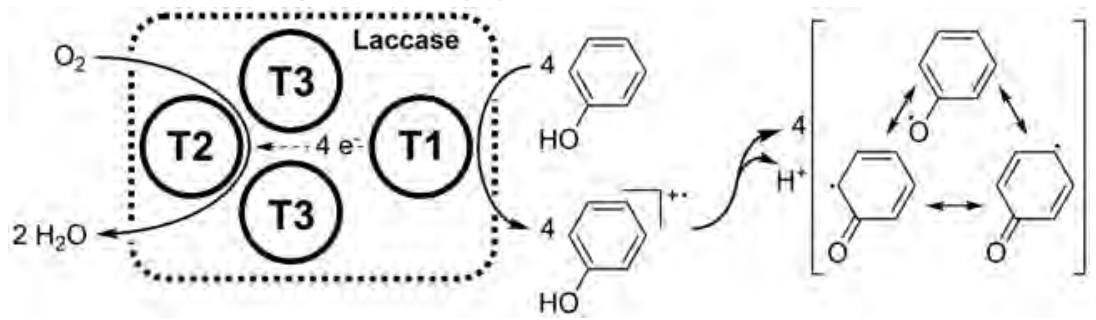

Scheme 3. Generic mechanism for the conversion of four phenolic substrates to their radical cations, with concomitant reduction of one molecule of dioxygen, followed by the rapid (non-enzymatic) loss of a proton to form the delocalised phenolic radical.

In general, three different types of copper form the active site of laccase. At the type 1 site (T1), four substrate molecules are converted to their radical cations. The thus extracted electrons are then transferred to the type 2 and type 3 sites (T2 and T3, respectively), at which molecular oxygen is reduced to two molecules of water. The formed substrate radical cations quickly lose a proton to form 
reactive radical species, which can then participate in non-enzymatic covalent bond formation or bond cleavage.

For small-molecule synthesis this bond formation can be easily studied by means of NMR spectroscopy or mass spectrometry. However, laccase-mediated grafting often results in the formation of a bond between two components of which at least one is a very complex matrix e.g. lignin. The formed bond is one of many in a very heterogeneous system; analysis of such an intricate architecture thus shows much resemblance to the infamous search for the needle in a haystack. In this search a covalent bond can easily be mistaken for (non-specific) adsorption. We postulate that covalent grafting using laccase is often claimed without posing substantial scientific evidence for it. In order to properly assess the likelihood of actual covalent bonding, the respective studies/methodologies are evaluated on the basis of five factors (F1-F5).

F1) Mechanistic rationale. Mixing two amino acids together at room temperature often does not result in peptide coupling; adding a carbodiimide will, however, result in amide-bond formation. ${ }^{\text {[25] }}$ More related to grafting: laccase oxidises (mostly) phenols, if these cannot be oxidised directly, a mediator might be required. ${ }^{[21]}$ Is laccase-mediated oxidation feasible with regard to the molecule's oxidation potential? Are the reactants reactive at the required $\mathrm{pH}$ ? These are some of the questions that arise when estimating the validity of the purported modification. Of course, grafting does not always have to proceed through the envisioned mechanism, which makes finding the imaginary needle even more challenging.

F2) Pre-treatment. Many of the materials discussed herein are of natural origin and could be contaminated with feculence or fats. ${ }^{[26]}$ Contaminants might shield the surface; thus hampering the intended grafting. Furthermore, (partially) soluble monomers or small oligomers of the material to be modified could be present in the material matrix. ${ }^{[27]}$ These molecules are likely to be more reactive than the molecules on the solid/liquid interface due to increased mobility and accessibility. ${ }^{[28]}$ Modification of these reactive species followed by precipitation onto the surface, could unintentionally suggest grafting. In some cases, pre-treatment involving cleaning or even extraction might therefore be required.

F3) Control experiments. Laccase-mediated grafting very often involves oligomerisation of phenols. ${ }^{[13 c]}$ Logically, the chemical and physical properties of the formed oligomers will be different from those of the parent monomer. Not observing the monomer in control experiments in which it is solely supplied to the surface, thus in the absence of laccase, does not necessarily confirm laccasemediated grafting. In a proper experimental design, the influence of oligomers on surface coating without laccase or with inactivated laccase should be assessed as well. Furthermore, lignin is rich in phenols, and therefore, also susceptible to laccase-mediated oxidation if no additional phenols are present. ${ }^{[29]}$ Additionally, proteins are notoriously fouling ${ }^{[30]}$; control experiments in which only laccase is applied on the surface (without additional monomers) are thus a necessity.

F4) Washing and cleaning. Proper washing after modification is at the essence of discrimination between adsorption and grafting, since this should remove the loosely bound adsorbents. The choice of washing solvent, additive and washing time might prove to be crucial, especially when oligomers are formed. These oligomers tend to be less soluble in the aqueous reaction medium; more apolar solvents are thus often advisable for cleaning. The simplicity of these steps has often not been combined with a proper estimation of their importance.

F5) Analytical tools. Arguably the most important step of grafting is the evaluation afterwards. Spectra are easily misinterpreted or, worse, misused. A direct indication of grafting would be the formation of a new unique covalent bond between the surface and the grafted molecule that has a distinct observational characteristic. Such indications are, however, typically rare. In most cases in which grafting is expected, simultaneous adsorption will also have occurred. Without the presence of a distinct observational characteristic, discrimination between adsorption and grafting can thus not (easily) be determined based on techniques such as FTIR spectroscopy and X-ray photoelectron spectroscopy (XPS). To conclude, without proper control experiments the mere observation of a 
molecule on the surface does not have to indicate grafting, since non-covalent adsorption often results in the same spectral alterations.

When all of the factors above have been considered and the resulting pre-requisites have been met, it is very likely that the presented methodology has resulted in covalent bond formation. However, considering all of the above only results in a binary statement: grafting or no grafting. It is essential to quantify the degree of grafting, the modes of grafting and the degree of adsorption. Additionally, in some applications (unintentional) leaching of adsorbents could result in contamination of the environment. ${ }^{[31]}$ In these cases, strong (covalent) binding is thus essential and adsorption should be reduced to a minimum. Furthermore, covalent binding does not have to indicate irreversible binding, that is, a reaction such as imine formation yields strong covalent bonds, but is known to be fully reversible. If the application requires strong binding, the grafting methodology should be tuned to meet those requirements.

The studies reviewed herein, in general, employ grafting in heterogeneous systems: dispersions, solid surfaces, undissolved solids etc. These are often the most difficult systems to analyse due to the limited solubility which induces identification errors. Additionally, all grafts discussed below arose from a grafting-to or a grafting-from approach. Laccase-mediated grafting-through strategies strongly resemble co-polymerisation, which is beyond the scope of this review. Each sub-domain will be individually discussed on the basis of the material upon which grafting is performed: lignocelluloses, polysaccharides, proteins and synthetic polymers. Per sub-domain the mechanistic foundations for grafting, the state-of-the-art regarding analysis of the corresponding grafts, and the pitfalls/challenges arising from grafting on these materials are discussed first. These are followed by specific examples of grafting that are, where possible, grouped according to application or employed methodology. Per example, we will elaborate on the factors determining the likelihood of grafting.

The goal of this review will thus not only be to outline the large numbers of excellent studies on the topic of laccase-mediated grafting, but also to compare them side by side to highlight either the lack or presence of considerable evidence for real covalent grafting.

\section{Laccase-mediated grafting on lignocelluloses}

\section{Validity of laccase-mediated grafting}

By appreciating that (fungal) laccases naturally act on lignin to convert it into useful feedstock for their host organism, ${ }^{[19 a]}$ it is not difficult to imagine that laccases are mainly employed in the modification of lignocellulosic material. ${ }^{[13 c]}$ Lignocelluloses comprise all natural fibres that are made up from lignin, hemicellulose and cellulose, albeit in varying ratios. ${ }^{[32]}$ This includes biomass, such as grasses and trees, value-added materials i.e. paper, pulp, cork and wood, but also waste generated when processing biomass into these materials. Although celluloses or other polysaccharides can also be modified (indirectly) by using laccase, the abundance of lignin in the lignocellulosic material is vital to the success of laccase-mediated grafting on lignocelluloses. ${ }^{[33]}$ The traditional removal of ligninderived chromophores from lignocelluloses to enhance whiteness (bleaching) by chlorine and peroxides is gradually being replaced by enzymatic methods, which includes the use of laccase. ${ }^{[34]}$ The mild way in which laccases generate radicals is employed in lignin polymerisation/oligomerisation, but also in delignification, ${ }^{[35]}$ an essential part of the bleaching process. This topic is beyond the scope of this review and excellently reviewed by Meyer and co-workers. ${ }^{[29]}$

The most common route towards lignocellulosic grafts, at first glance, provides a clear mechanistic rationale (Scheme 4): a laccase-generated phenolic radical (initiation) reacts with an oxidisable moiety (propagation) or another radical (termination). However, the free radical, be it from a phenol in solution or from lignin, is delocalised over the aromatic system, which often implicates multiple reactive sites. Coupling of such delocalised radicals thus results in diverse substrate-lignin, substratesubstrate and even lignin-lignin dimers and subsequent oligomers. Achieving a solid mechanistic 
understanding of these processes is further complicated by the presence of phenolic extractives in the lignocellulosic matrix, as these extractives could also act as laccase substrates. Additionally, applying mediators to the system further alters the reaction pathway. Its highly diverse nature thus clearly indicates the complexity of the laccase-mediated modification of lignocelluloses.

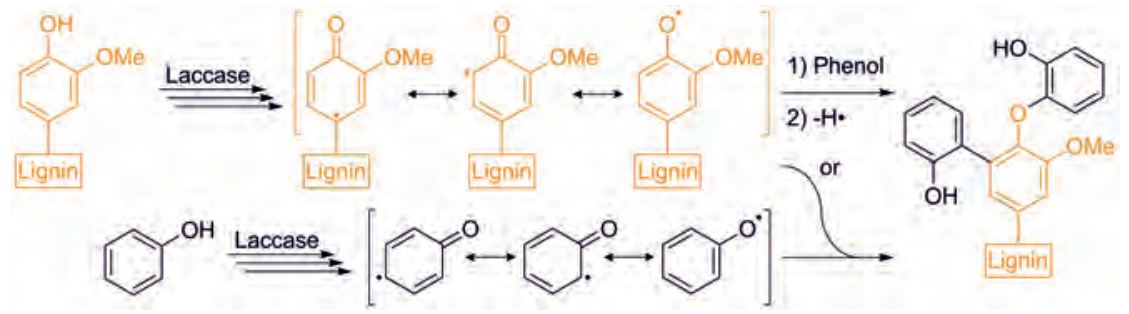

Scheme 4. Generalised schematic of laccase-mediated grafting of phenol on lignin.

In order to understand what happens in a complex system, such as wood, one often reverts to lignin model compounds; small "monomers" of the highly complex lignin structure that contain many of the functional groups present in lignin. These monomers, however, are generally soluble in aqueous buffer/organic solvent mixtures, and have limited complexity, so they can be separated and studied by both LC-MS and NMR spectroscopy. The groups of Nyanhongo and Guebitz (vide infra) have extensively studied lignin model compounds and their interaction with laccase. In early studies, beech wood, laccase and tyramine (Scheme 5A) were reacted together to result in a depletion of tyramine ${ }^{[36]}$ even though tyramine itself is not a substrate for the laccases employed therein due to its high oxidation potential. The influence of beech wood is confirmed when the lignin model adlerol (Scheme 5B) is used instead of wood; since adlerol is also not a laccase substrate, no depletion of tyramine was observed. In all cases where phenolic lignin model compounds were used, tyramine was converted (Scheme 5C).

(A)<smiles>NCCc1ccc(O)cc1</smiles>

(B)

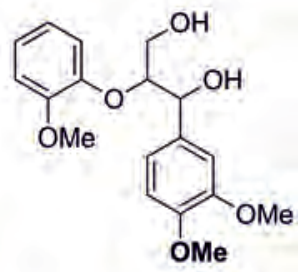

(C1) \& (C2)

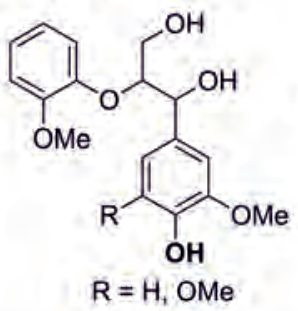

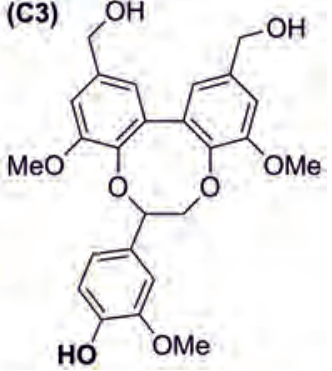

Scheme 5. A: Tyramine, B: non-phenolic lignin model compound adlerol and, C: phenolic lignin model compounds $\mathrm{C1}$ : guaiacylglycerol $\beta$-guaiacylether $(\mathrm{R}=\mathrm{H}), \mathrm{C}$ : syringylglycerol $\beta$-guaiacylether $(\mathrm{R}=\mathrm{OMe})$ and $\mathrm{C} 3$ : dibenzodioxocin.

"Grafting" on model compounds was later confirmed by studying the coupling of lignin models to several functional molecules by LC-MS. ${ }^{[37]}$ In all cases in which a phenolic molecule was reacted in the presence of the phenolic lignin model dibenzodioxocin (Scheme $5 \mathrm{C} 3$ ), the heterodimer $[\mathrm{M}+\mathrm{H}]^{+}$ion of the model/phenol conjugate was observed. In this way, the lignin model compound was conjugated to tyramine, tyramine derivatives or molecules bearing a fluorine probe. The use of fluorine-substituted phenols was further exploited towards the hydrophobisation of beech veneers. ${ }^{[38]}$ The authors clearly recognised the possible interference of extractives, since the wood was Soxhlet-extracted with acetone prior to modification. Depending on the fluorinated phenol that was grafted, the static water contact angle (SWCA; an indirect measurement of surface hydrophobicity) increased from $54^{\circ}$ for unmodified wood to up to $90^{\circ}$ for grafted wood. Observation of the coupling between the fluorinated phenol and a model compound through NMR spectroscopy was posed as additional theoretical evidence for the plausibility of grafting on lignocelluloses. 
In additional studies, the efficiency of grafting by other laccases was examined. It was shown that, the use of laccase from Bacillus SF spores resulted in coupling of syringylglycerol $\beta$-guaiacylether and tyramine through $\mathrm{C}-\mathrm{O}$ bond formation rather than through $\mathrm{C}-\mathrm{C}$ bond formation as the ortho positions (with respect to the phenyl alcohol) were blocked (Scheme 6, P1). ${ }^{[39]}$ Further transformations included dehydroxylation (P2), oxidation (P3) and nucleophilic attack by either of the solvents water (P4) or methanol (P5); which has been shown to be rather common in laccase-mediated oxidation. ${ }^{[40]}$<smiles>COc1ccccc1OC(CO)C(c1cc(OC)c(Oc2cc(CCN)ccc2O)c(OC)c1)C(C)(C)C</smiles>

P2, dehydroxylation<smiles>COc1ccccc1OC(CO)C(=O)c1cc(OC)c(Oc2cc(CCN)ccc2O)c(OC)c1</smiles>

P3, oxidation<smiles>COc1ccccc1OC(CO)C(O)c1cc(OC)c(Oc2ccccc2OC(CO)C(O)c2cc(OC)c(Oc3cc(CCN)ccc3O)c(OC)c2)c(OC)c1</smiles>

P1, coupling<smiles>COc1ccccc1OC(CO)C(O)c1cc(OC)c(Oc2cc(CCN)cc(O)c2O)c(OC)c1</smiles>

P4, Nucl. $=\mathrm{H}_{2} \mathrm{O}$<smiles>COc1ccccc1OC(CO)C(O)c1cc(OC)c(Oc2cc(CCN)cc(OC)c2OC)c(OC)c1</smiles>

P5, Nucl. $=\mathrm{MeOH}$

Scheme 6. Coupling of syringylglycerol $\beta$-guaiacylether and tyramine to form P1 followed by subsequent chemistries. P2-P5 are representative structures for the set of possibilities related to the mentioned reaction (based on Kudanga and co-workers). ${ }^{[39]}$ Nucl.: nucleophile.

Apart from grafting phenolics from lignin, Kudanga and co-workers also managed to graft and concomitantly hydrophobise beech veneers by using alkylamines. ${ }^{[41]}$ To support their hypothesis that covalent bonding, and not mere adsorption, was responsible for the increase in hydrophobicity, the authors again reverted back to simple phenolics and lignin model compounds (Scheme 7A/B). Reacting either catechol or guaiacol with laccase in the presence of $n$-dodecylamine resulted in mono-, diand/or trimeric species coupled to exactly two $n$-dodecylamine molecules (Scheme 7C). A similar coupling pattern (phenol/amine $=1: 2,2: 2$ and/or 3:2) was observed for di- $n$-hexylamine. Upon coupling $n$-dodecylamine with lignin model compounds, 1:1 coupling products between the guaiacoltype lignin model compounds and the amine were observed (Scheme 7D).

As follow-up, the beech veneers were reacted with laccase in the presence of either amine to afford highly hydrophobic beech wood (increase in SWCA from $58^{\circ}$ (unmodified beech) to 107 and $81^{\circ}$ ). Furthermore, extensive washing and rinsing with a good solvent (with respect to the amines) assured that any adsorbed material was washed off. Similar results were later found for the modification of jute fabrics with octadecylamine. ${ }^{[26]}$ The importance of extraction prior to laccasemediated modification was again exemplified because non-extracted and unmodified jute exhibited static water contact angles of $111^{\circ}$, due to the presence of lipophilic wax and fats. 


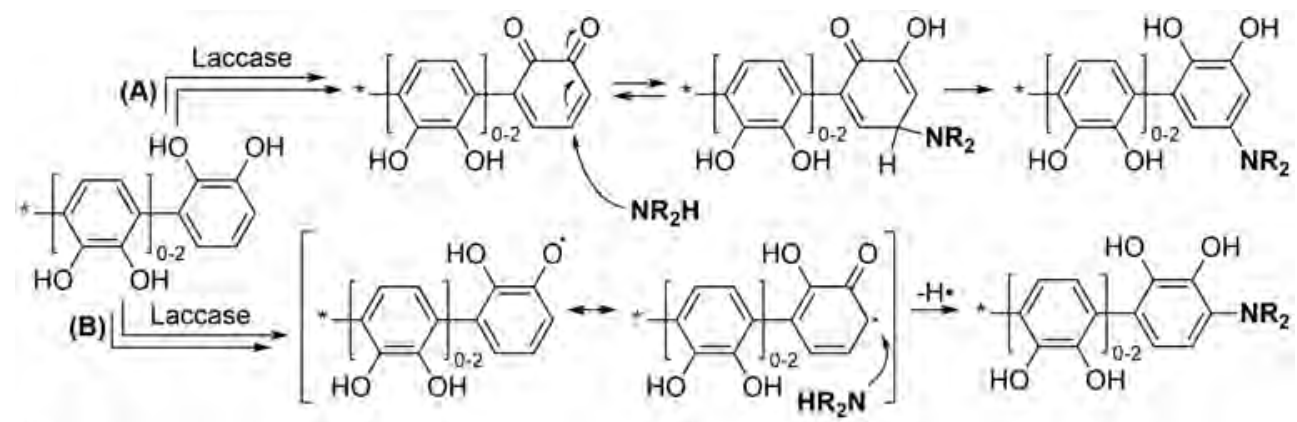

(C)

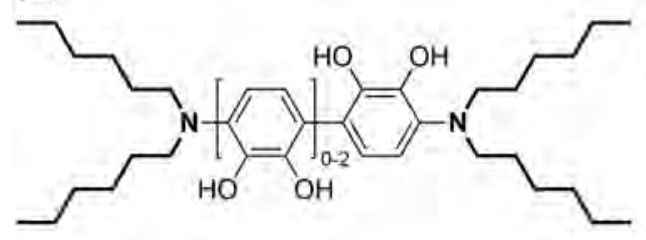

(D)

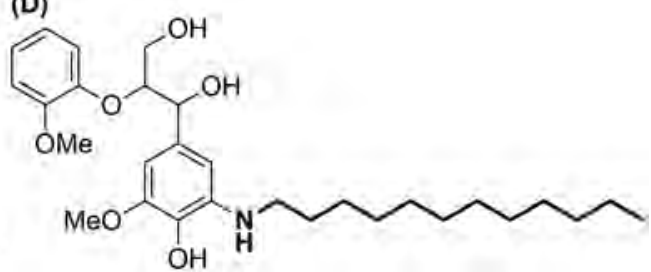

Scheme 7. A: Mechanism for the coupling of alkylamine to a laccase-generated quinone via Michael addition. B: C-N bond formation through coupling of an amine and a phenolic carbon radical. C: Catechol/di- $n$-hexylamine 1:2, 2:2 and 3:2 conjugates. D: Guaiacylglycerol $\beta$-guaiacyl ether $n$-dodecylamine 1:1 conjugate (based on the work by Kudanga et al. $\left.{ }^{[41]}\right)$.

Although the use of model compounds to mimic bigger polymeric matrices provides a powerful tool to study grafting reactions on a molecular level, it also has its limitations. Lignin is a highly complex network composed of many types of functionalities. In order to simplify this system to an extent that NMR spectroscopy or LC-MS-based techniques are viable, one is thus always limited to only a selection of functional groups per model compound. This implies that, in order to properly mimic lignin, a multitude of fragments is required. Additionally, approximately $10-20 \%$ of lignin is comprised of phenolic subunits, while the model compounds used by Kudanga and co-workers are 19-28\% phenolbased, which means that any laccase reactivity will be an overestimation. ${ }^{[42]}$ This discrepancy is accentuated further by appreciating the fact that the model compounds are soluble and small enough for all phenols to be oxidised by laccase, while only some of the phenols on the exterior of the insoluble lignin will be accessible to laccase. Furthermore, in a homogeneous mixture, as is the case for model compounds, the chances for the model compound and graftable molecule to react are far higher than those in the case of insoluble lignin. A phenolic lignin surface radical is likely to be quenched by a nearby lignin moiety or other unintentional scavengers present in the reaction mixture. Any representative set of lignin model compounds would thus not be complete without a non-phenolic and insoluble molecule (that could be made soluble upon analysis).

Apart from the mentioned bottom-up approach using lignin model compounds, several researchers have aimed at utilising pyrolysis-coupled-to-GC-MS analysis (Py-GC-MS) to study grafting in a topdown fashion. Using this methodology, Vidal et al. studied the laccase-mediated modification of flax and sisal pulps in the presence of a range of simple phenols. ${ }^{[43]}$ Initial measurements revealed that kappa numbers (an indication of lignin content) most notably increased for flax treated with $p$ coumaric acid. Laccase-induced sisal pulp treatment with guaiacyl-type (G-type) phenolics, such as ferulic acid (FA) or coniferaldehyde, resulted in higher kappa numbers than treatment with syringyltype (S-type) phenolics did (Scheme 8). This was rationalised by assuming more steric hindrance around the phenyl alcohol, which is surrounded by two methoxy substituents, rather than one. Additionally, the C2-carbon without a methoxy substituent bears high electron density in the phenolic radical. ${ }^{[44]} \mathrm{G}$-type phenolics thus have not one, but two reactive sites, and therefore, the chance of covalent coupling is increased. Subsequently, the modified and acetone-extracted sisal and flax fibres 
were subjected to Py-GC-MS analysis in the presence of tetramethylammonium hydroxide (TMAH) as base and methylating agent (Scheme 9). The observation of the 3,4-dimethoxycinnamic acid methyl ester confirmed the presence of FA after laccase-mediated sisal modification. Similar results were observed for the modification of flax fibres. Assuming that all adsorbed phenol had been extracted off, this would thus imply that grafting had really taken place.

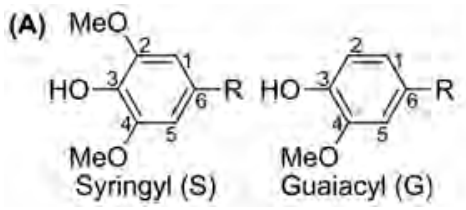<smiles>COc1cc(O[14CH3])ccc1C1OC(=O)C2C(c3ccc(O[14CH3])cc3OC)OC(=O)C12</smiles>

Scheme 8. A: Syringyl/guaiacyl nomenclature and B: ferulic acid dilactone graft as envisioned by Rencoret et al.[45]

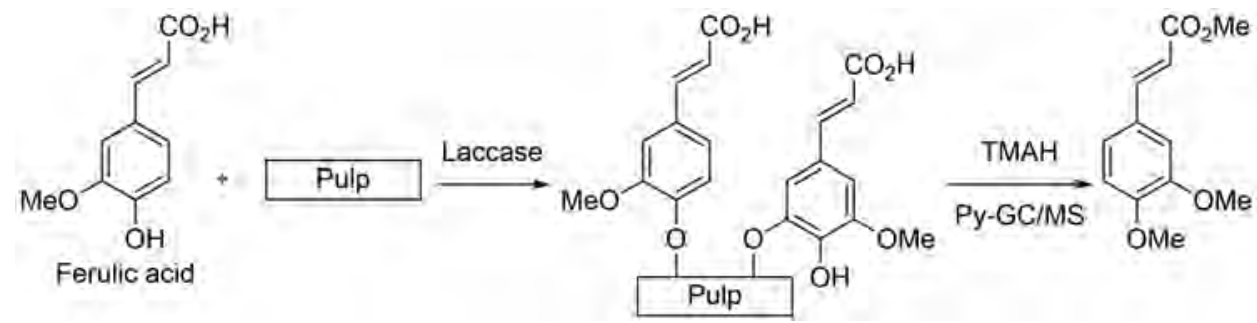

Scheme 9. Simplified schematic representation for the laccase-mediated grafting of ferulic acid (FA) from pulps followed by Py-GC-MS analysis with methylating agent TMAH (the depicted pulp-FA graft is merely an indication of the possible structure; many more structures possible, based on the work by Vidal and co-workers). ${ }^{[43]}$

As a follow-up, the laccase-mediated modification of sisal pulp with FA was studied in more detail. ${ }^{[45]}$ The formation of an etherified ferulic acid derivative is claimed on the basis of a $1 \mathrm{ppm}$ shift in the ${ }^{13} \mathrm{C}$-spectrum of isolated modified lignin. However, caution has to be exerted when interpreting NMR spectra of complex lignocellulosic grafts. Upon grafting phenolics from lignin, the formed bonds are so similar to those present in the native lignin that differentiation between the two is highly challenging. Nonetheless, a plausible dilactone originating from FA dimerisation could also be observed (Scheme 8B). Although both grafting of the monomer and the dilactone are highly likely, (2D) NMR spectroscopy performed in this way could, in our opinion, not unambiguously confirm covalent binding to the lignin backbone. As mentioned, employing mediators further enlarges the scope of laccasemediated oxidation reactions. However, one should be aware of the type of chemistry that is introduced with the use of these mediators. A mediator, such as veratryl alcohol, for example, only acts as a one-electron shuttle from laccase to a non-laccase substrate, while $\mathrm{N}$-O radicals like $(2,2,6,6-$ tetramethylpiperidin-1-yl)oxyl (TEMPO, Scheme 10) are oxidised by laccase to the corresponding oxoammonium cation, which can perform two-electron oxidations. ${ }^{[46]}$ Using four different mediators (2,2'-azino-bis(3-ethylbenzothiazoline-6-sulphonic acid (ABTS), 1-hydroxybenzotriazole (HOBt), Nhydroxyphthalimide (HPI) and TEMPO, Scheme 10) and two types of lignin (wheat straw lignin and beech organosolv lignin), Munk et al. further exploited the use of Py-GC-MS to study the effect of laccase-mediator treatments on lignin. ${ }^{[47]}$ Grafting could only be observed for HOBt and HPI, while grafting of HPI was substantially more efficient (Table 1 ). This difference was explained by taking into account that the half-lifetime of the HOBt radical is approximately 800 times shorter than that of the HPI radical. The HPI radical, therefore, has more time to "find" the insoluble lignin. Grafting from beech organosolv lignin (BOL) was relatively less efficient, compared to grafting from wheat straw lignin (WSL). This could be rationalised by appreciating the fact that the S/G ratio of BOL was approximately five times higher than that of WSL. Additionally, the high lignin content of BOL implies that the core lignin is almost inaccessible. Furthermore, pre-treatment of organosolv lignin during production is known to result in less reactive C-C bonds. Actual covalent grafting is made highly likely as no mediator was observed by Py-GC-MS analysis in control experiments without laccase, and 
laccase-mediated oligomerisation (and subsequent adsorption) of these mediators is not probable. Although unlikely, one should be aware of possible other adsorption mechanisms via mediatoroligomers or mediator/laccase complexes that would not occur in the control experiments mentioned above.

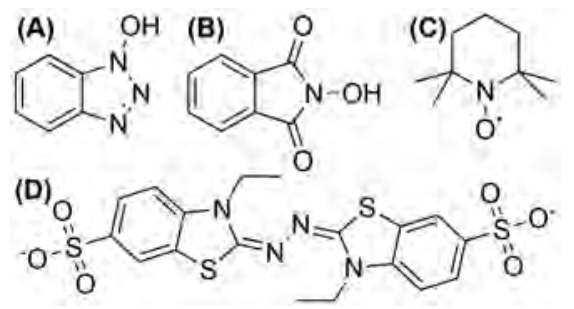

Table 1. Grafting yields for mediator grafting on lignin.

\begin{tabular}{lcccc}
\hline S:G & Lig. $^{[\mathrm{b}]}$ & $\begin{array}{c}\text { HOBT G\% } \\
\text { (100 s) }\end{array}$ & $\begin{array}{c}\text { HPI G\% } \\
\text { (7900 s) }\end{array}$ \\
WSL & 0.6 & 43.7 & $5-6$ & $32-35$ \\
BOL & 2.7 & 87.4 & 1 & 11 \\
\hline
\end{tabular}

[a] Syringyl-type (S)/guaiacyl-type (G) lignin ratio for unmodified lignin. [b] wt\% lignin. [c] Grafting yield in percentages (half-lifetime of mediator radical).

Scheme 10. Mediators A: 1-hydroxybenzotriazole (HOBt), B: N-hydroxyphthalimide (HPI), C: TEMPO and D: 2,2'-azino-bis(3-ethylbenzothiazoline-6sulfonic acid (ABTS).

These studies have shown that Py-GC-MS is a promising tool for examining laccase-mediated grafting because the grafted moiety can be detected directly. Unfortunately, one has to rely on the efficiency of the washing procedure to ensure substantial removal of non-covalently bound material. If pyrolysis, or any breakdown technique for that matter, can be balanced in such a way that internal lignin bonds between the grafted moiety and lignin are also broken, it might provide an even better view of the molecular nature of the lignin graft. In addition, 2D NMR spectroscopy has proven to aid lignin characterisation to a huge extent. ${ }^{[48]}$ If the grafted molecules are chosen in the right way $2 \mathrm{D}$ NMR spectroscopy may also be the optimal tool to study grafting reactions on lignin.

When studying laccase-mediated grafting, control experiments have to be designed with care. Very often laccase-mediated modification is only compared to the simple addition of the monomer (without laccase). However, apart from laccase-induced grafting on the surface, laccase also oligomerises phenolic monomers in solution. The physical and chemical properties, such as solubility and adsorption tendency, of these oligomers will be different to those of the monomeric species itself. In earlier work by Kim and co-workers, this issue was partially addressed by comparing in-situ colouration of flax fibres to colouration with oligomers that had been generated ex-situ (from morin and quercetin; Scheme 11) in a two-step one-pot process. ${ }^{[49]}$<smiles>O=c1c(O)c(-c2ccc(O)cc2O)oc2cc(O)cc(O)c12</smiles><smiles>O=c1c(O)c(-c2ccc(O)c(O)c2)oc2cc(O)cc(O)c12</smiles>

Scheme 11. A: Morin and B: quercetin.

Although laccase was not deactivated prior to the second step, laccase activity will be limited because most monomers and short oligomers small enough to enter the active site will have reacted in the first step. However, in the case of quercetin, flax fibres were equally well coloured with both procedures. This could indicate two things: either the formed oligomers are still small enough to be oxidised by laccase and are thus able to graft from the surface, or colouration is mainly affected by adsorption of oligomers. However, when using morin, different results were obtained. In this case, the two-step colouration was less effective than that of the one-step process. Since morin has a higher oxidation potential (more difficult to oxidise/initiate oligomerisation) than quercetin does, adsorption phenomena are relatively more likely to contribute to colouration. Furthermore, colour strength still increased when the temperature in the second step was increased to 80 or $90{ }^{\circ} \mathrm{C}$, under which conditions laccase is inactive. ${ }^{[50]}$ This is thus an extra indication that adsorption is likely to play a role. On the other hand, flax fibres subjected to a bleaching treatment prior to modification showed less 
colouration. This might indicate that lignin content was further depleted and less sites were available for covalent attachment of phenolics. These results specify that adsorption phenomena play a role during laccase-mediated grafting, and that proper control experiments can indicate to what extent.

To summarise: the tools mentioned above (lignin model studies and Py-GC-MS), together with a reliable mechanistic background, adequate pre-treatment, appropriate control experiments and sufficient washing procedures, have provided a solid foundation for the ability of laccase to graft phenolics, alkylamines and certain mediators covalently on lignin. In the studies discussed below, we will thus often assume that covalent grafting is possible. We will, however, highlight those instances in which it is unclear whether covalent grafting is the main mode of functionalisation, as in the case of insufficient washing, for example.

\section{Examples of grafting}

Mechanical strength, hydrophobicity, anti-microbial activity or anti-oxidant activity are the properties studied most frequently when aiming to improve chemical or mechanical stability of lignocelluloses by laccase-assisted treatments. Enriched paper hand sheets made from softwood Kraft pulp subjected to a laccase treatment in the presence of several phenolic monomers, as developed by Orlandi and co-workers, is one of many examples. ${ }^{[51]}$ In almost all cases, the modified hand sheets were proven to diminish bacterial growth, in comparison to that of unmodified paper. The most notable effects were found with caffeic acid, 4-hydroxybenzoic acid and dopamine oligomers, which induced bactericidal activity against multiple bacterial strains. Although grafting is highly likely to contribute, simple adsorption could not be ruled out since the modified sheets were only washed with water after the enzymatic treatment.

Laccase-mediated functionalisation of wood veneer and pulp using tannins (natural polyphenols) conferred anti-bacterial properties to the wood. ${ }^{[52]}$ Although simple phenols proved to reduce bacterial growth to a higher extent, tannic acid-modified lignocelluloses almost completely diminished the growth of S. aureus. Additionally, chestnut tannin and cationic tannin, in particular, induced a substantial anti-bacterial effect against $E$. coli. Coupling between tannin base-units (catechins) and simple phenolics as lignin model compounds mainly resulted in 1:1-coupled conjugates.

Oligomers originating from laccase-mediated coupling of phenols are known to be strongly coloured due to their extended $\pi$ systems. Schroeder et al. coloured flax fibres through in-situ laccaseassisted oligomerisation of several phenolic monomers. ${ }^{[53]}$ However, most likely due to limited washing with water only, colour fastness was low. Modification did, nonetheless, result in growth inhibition of B. subtilis and S. aureus (at 6 and $4 \mathrm{mM}$ respectively) by the orange-coloured FA-coated surface. The minimum inhibitory concentration for the white hydroquinone surface against these bacterial strains was even below the detection limit.

In order to minimise biodeterioration and oxidation of linen, Silva and co-workers pre-treated flax fibres with laccase, followed by coupling with catechin or chitosan, or first chitosan, followed by catechin (Scheme 12). ${ }^{[54]}$ Prior oxidation with laccase proved to be essential to enhance colour strength when this was followed by a catechin/laccase treatment; without pre-treatment, colour strength was two to five times lower. The authors indicate more grafting on the surface as the cause of higher colour strength due to the presence of, pre-treatment induced, reactive quinones that are susceptible to radical coupling. Grafting of chitosan on the pre-treated linen in the presence of laccase, followed by laccase-mediated grafting of catechin, however, resulted in poor fixation of catechin. Amine groups of chitosan likely bound the pre-formed quinones generated from bare flax lignin covalently through, previously mentioned, radical coupling, Michael addition (Scheme 7) or imine formation. In all cases, less amine groups would have been available to couple with oxidised catechin in the subsequent step. Both the chitosan/catechin- and catechin-modified linen showed excellent antioxidant properties compared with those of control linen only treated with chitosan and/or catechin (no laccase). 


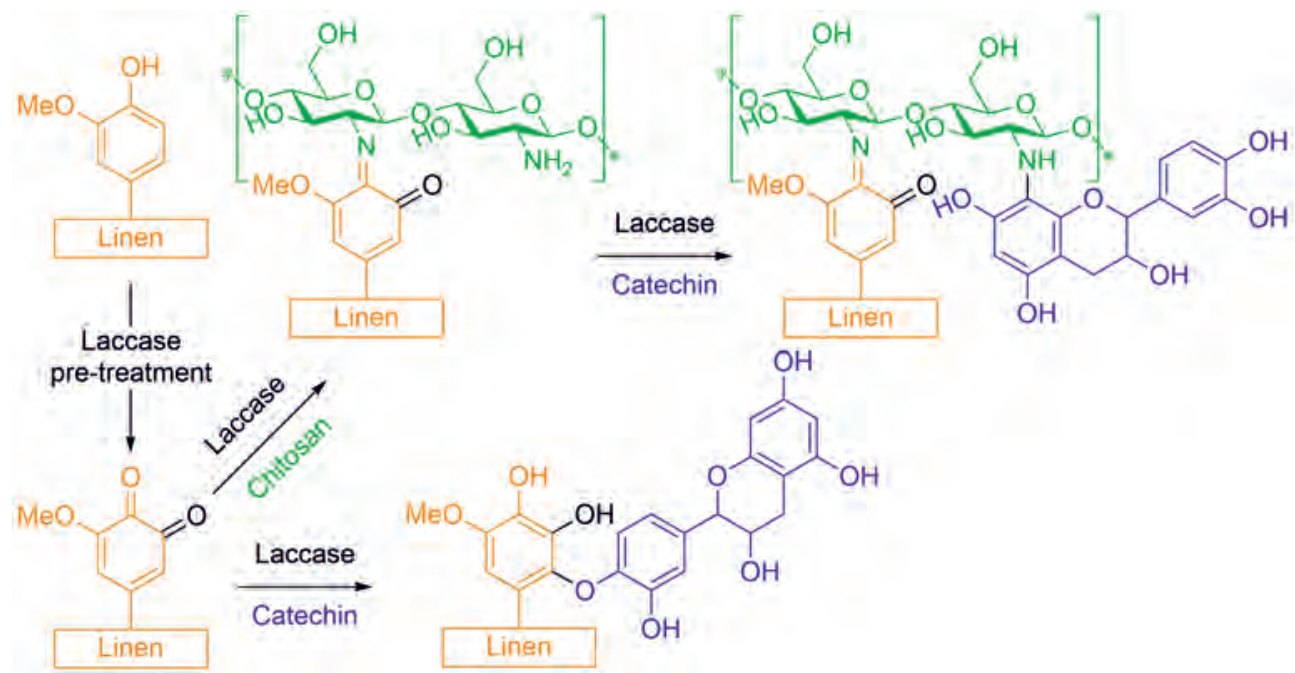

Scheme 12. Reaction scheme for the modification of linen through a laccase-mediated chitosan, catechin or chitosan/catechin treatment with or without laccase pre-treatment (depicted structures are merely an indication of the molecular nature of the graft; many more structures are possible, based on Silva and co-workers). ${ }^{[54]}$

Roncero and co-workers reacted simple phenols directly with laccase in order to confer antibacterial properties to flax pulp. Almost complete growth reduction of several bacterial strains was achieved for paper made from pulps treated with laccase and $p$-coumaric acid at initial phenol concentrations of $15 \mathrm{mM}$. However, laccase-mediated modification with syringaldehyde or acetosyringone only resulted in substantial growth inhibition of Klebsiella pneumoniae at concentrations above $20 \mathrm{mM}$. Once again, the treated pulp was only washed with water, so deposition of less soluble phenol oligomers could be responsible for the effects obtained. ${ }^{[55]}$

Traditionally, conferring anti-microbial activity to lignocellulosics using laccase is performed through the grafting of phenolic compounds. Schubert et al., however, employed laccase's ability to oxidise iodide in order to iodinate lignin to enhance the anti-microbial activity of spruce wood. ${ }^{[56]}$ The growth of E. coli, S. aureus and Saccharomyces cerevisiae was completely inhibited after iodination at an initial iodide concentration of $50 \mathrm{mM}$ with the addition of the mediator ABTS. Additionally, antifungal activity was assessed after iodination by comparing the mass loss of treated and untreated samples caused by the wood-digesting fungi Oligoporus placenta and Trametes versicolor. Wood treated at an initial iodide concentration of $50 \mathrm{mM}$ in the presence of laccase (with or without mediator acetosyringone) was completely resistant to mass loss caused by both fungi. Anti-fungal activity was thereby as high as with commercial fungicide VP 7/260a. Applying iodide only was significantly less effective against both bacterial growth and fungal degradation in all cases. Substantial and repeated leaching procedures using water were applied in all mentioned cases, which should be sufficient to leach out all formed and unbound $I_{3}{ }^{-}$. FTIR analysis was used to assess chemical alterations in the modified wood. Apart from observing structural changes after grafting, no direct proof of iodination was obtained. FTIR spectroscopy could have been used more effectively in this case, since C-I stretching vibrations, which are not present in unmodified wood, would be observed as a strong signal at $\bar{v}=500 \mathrm{~cm}^{-1}$. As a benchmark for their iodination strategy, Schubert and co-workers also assessed the anti-bacterial effect induced by phenol grafting rather than iodination. It was observed that wood treated with thymol or isoeugenol alone resulted in a significant growth reduction of multiple bacterial strains. However, when laccase was added to the reaction mixtures, only the laccase/isoeugenol treatment conferred anti-bacterial activity to the wood, and only for one bacterial strain (S. aureus). Apparently grafting, and/or oligomerisation and adsorption of thymol, had led to a loss of the antibacterial properties. The authors have subjected the wood to extensive washing with water, but it is 
unclear how effective this is considering the poor solubility of the phenols and its oligomers in water. Orlandi and co-workers, however, obtained different results: ${ }^{[51]}$ in their hands, isoeugenol was not an effective anti-bacterial agent against S. aureus and thymol only moderately so, but wood pulp treated with laccase/isoeugenol was bactericidal. In all cases, the effect is less than that with the iodination approach of Schubert et al., ${ }^{[56]}$ which shows that iodination is a more efficient, but underappreciated, method to confer anti-microbial activity to lignocellulosic material.

Tzanov and co-workers developed a silver nanoparticle (NP)-cork composite as an anti-bacterial adsorbent for wastewater treatment (Scheme 13). ${ }^{[57]} \mathrm{AgNO}_{3}$ was firstly reduced to $\mathrm{Ag}$ in the presence of an amine-functionalised carbohydrate (chitosan (CS) or a 6-deoxy-6-( $\omega$-aminoethyl)amino cellulose derivative (AC)), followed by laccase-assisted fixation of the NP to cork. Cork consists of lignin, tannins and suberin (a polyester containing long chain fatty acids, hydroxy fatty acids and phenolic acids), which are susceptible to laccase-mediated oxidation. ${ }^{[58]}$ The cork-AgNP-CS composite fully inhibited the growth of $E$. coli and S. aureus, whereas the AC-composite was only able to completely inhibit the growth of $S$. aureus. Although substantial grafting can be expected, washing after modification was performed with water, which might not have removed all adsorbed insoluble NPs. In order to test the anti-bacterial efficiency during use, water filtration cartridges were filled with the cork-AgNP-CS composite material. ${ }^{[59]}$ All bacteria could be removed from the effluent at $8 \mathrm{~h}$ residence time. However, approximately $25 \%$ of the total silver content had been leached out after five disinfection cycles. This would contaminate the water and diminish the composite's anti-bacterial effect, but, additionally the question arises to what extent the leached silver contributes to the anti-bacterial effect.

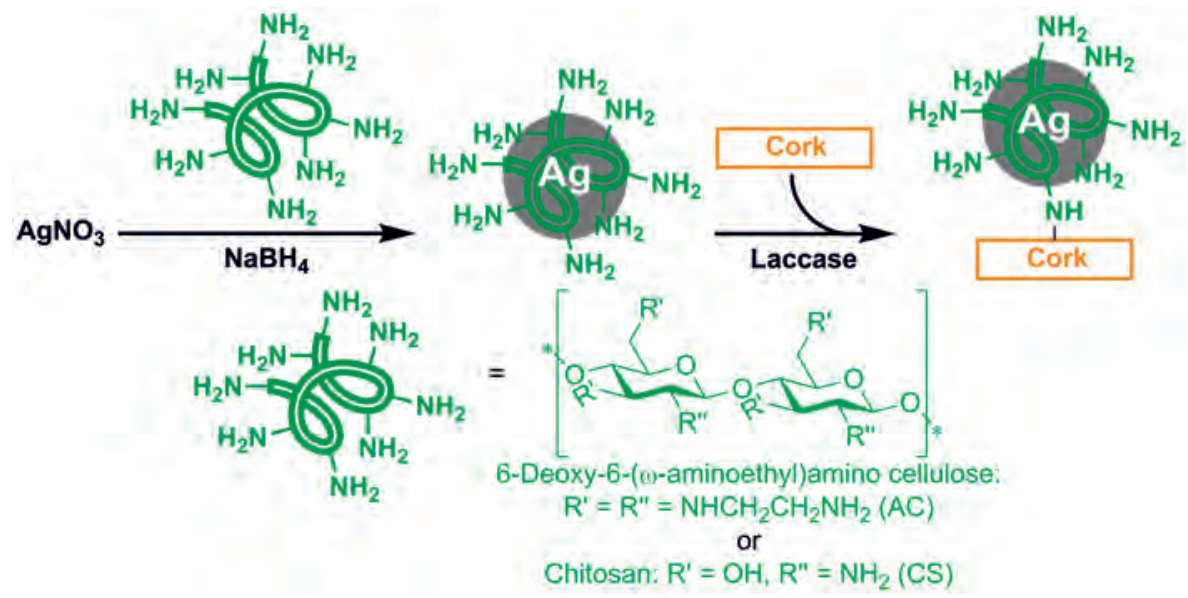

Scheme 13. Reaction scheme for the synthesis of an anti-bacterial silver nanoparticle-cork composite (based on Tzanov et al. ${ }^{[57]}$ ).

Natural sources are exploited more and more as a basis for composite materials. Unfortunately, these materials often suffer from low compatibility with man-made plastics due to their high hydrophilicity. In order to enhance its hydrophobicity, Fan et al. modified jute with laccase in the presence of dodecyl gallate (Scheme 14). ${ }^{[60]}$ Soxhlet-extraction with acetone was expected to ensure removal of any unbound material after grafting. Static water contact angle (SWCA) measurements revealed an increase from $30^{\circ}$ for unmodified material to $111^{\circ}$ for modified material. Furthermore, although control experiments with laccase or gallate only, also resulted in higher SWCA's, these droplets wetted the fibres within $1 \mathrm{~min}$, while the SWCA for the laccase/gallate-modified jute stayed practically unchanged for the first $5 \mathrm{~min}$. FTIR analysis was used to indicate whether gallate or gallate oligomers were covalently bound to the jute. The appearance of new bands, however, does not necessarily indicate the formation of a bond between the surface and the graftable moiety. To aid analysis, one should at least perform control experiments in which the phenolic oligomers are formed ex-situ and subsequently deposited on the surface. But, even then, the formed bonds are very similar 
to those in the lignin matrix, especially when grafting phenols, which makes FTIR analysis at best highly complicated, and often an unreliable tool. Modified jute/polypropylene composites exhibited higher breaking strengths than the composites with native jute. As a follow-up, also propyl and octyl gallate were used as jute modifiers in the presence of laccase. ${ }^{[61]}$ As expected, these molecules were less efficient at conferring hydrophobicity to the jute. These new materials thus showed non-optimal compatibility with polypropylene, which was apparent from a lower tensile strength of these composites, than that of the dodecyl gallate jute/polypropylene composites.

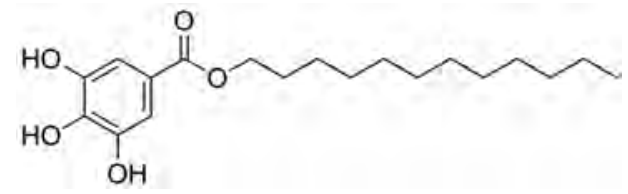

Scheme 14. Dodecyl gallate.

Lignocelluloses with higher lignin contents, such as beech wood, could also be made hydrophobic through in- situ laccase-assisted dodecyl gallate oligomerisation and grafting. ${ }^{[62]}$ Apart from the wood being hydrophobic (SWCA $>90^{\circ}$ ), the presence of dodecyl gallate (oligomers) after acetone extraction was also confirmed by FTIR and XPS analysis. On the basis of a decrease in the surface, oxygen/carbon ratio, covalent attachment was claimed; however, non-covalent deposition of gallate oligomers would cause a similar decrease. So, although XPS analysis can be a powerful tool, as with FTIR measurements, one should be careful with its interpretation.

Garcia-Ubasart and co-workers developed a simple method to make paper more resistant to wetting by reacting pulp with dodecyl gallate and laccase. ${ }^{[63]}$ Although other phenols were also investigated, dodecyl gallate was the only compound that could substantially increase the wetting time of the paper. Unfortunately, the mechanical strength of the treated paper suffered from the conditions under which the highest degree of grafting could be expected (higher laccase/gallate concentrations and longer reaction times). Later studies further confirmed wetting resistance by SWCA measurements. ${ }^{[64]}$ Furthermore, an increase in kappa number (an indication of the degree of lignin/phenolics) was observed after modification and subsequent acetone extraction. The exact mechanism behind laccase-mediated functionalisation of lignocelluloses in the presence of gallates has, however, yet to be clarified. Studies have shown that under some conditions the kappa number increases after laccase/gallate treatment, but drops again after extraction. After modification of lignocellulosic material with a similar lignin content as the jute and wood mentioned above (> 5\%), Reynaud et al. observed that after extraction a drop in kappa number coincided with a decrease in SWCA and an increase in water absorption rate (back to the level of unmodified material). ${ }^{65]}$ During lignocellulosic processing, some xylan monomers are converted into hexenuronic acids (HexA's, Scheme 15); undesired olefinic chromophores that cause aging of the lignocelluloses. Laccase/mediator treatments are known to decrease the HexA content of the material; therefore, Cadena and co-workers tested $p$-coumaric acid and dodecyl gallate as mediators for the removal of HexA. ${ }^{[33]}$ The use of dodecyl gallate in the presence of Trametes villosa laccase was indeed very effective in removing HexA ( $82 \%$ decrease), while a Pycnoporus cinnabarinus laccase/ $p$-coumaric acid treatment removed only $2.8 \mu \mathrm{mol} \mathrm{g}^{-1}(22 \%)$ of the HexA present in the unbleached flax pulp (lignin content $1.4 \%$ ). Unlike what Reynaud and co-workers observed, the kappa number was approximately twice as high after modification and subsequent acetone extraction, even though a low lignin content flax was used. ${ }^{[65]}$ Moreover, when lignin-free and HexA-free pulp were used, almost no change in kappa number was detected. However, when lignin-free pulp with high HexA content was modified, the approximate twofold increase in kappa number was again observed. It was thus postulated that grafting does not occur through coupling with lignin, but through coupling with HexA. These remarkable results have yet to be confirmed, but laccase-assisted modification of gallates in the presence of a HexA model compound might reveal more details on the grafting process. 


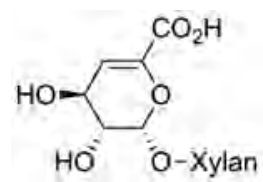

Scheme 15. Xylan-coupled hexenuronic acid (HexA).

Witayakran and co-workers employed laccase-mediated oxidation of softwood Kraft pulp to functionalise it with amino acids. ${ }^{[66]}$ Imine formation, radical coupling or Michael addition on the generated quinones would likely result in covalent grafting. When histidine was used, the tensile strength, tear strength and wet tensile strength of paper made from these pulps improved. Measuring carboxylic acid content was used to demonstrate the presence of amino acids. However, treating the pulp with laccase only, or amino acids only, also resulted in an increase in carboxylic acid content. Although the carboxylic acid content after laccase/amino acid treatment was higher than that of the samples treated with either laccase or amino acids alone, this could thus simply result from the adsorption of both.

Apart from commonly observed nucleophilic coupling of amines or grafting of phenols, also functionalisation with methacrylate or acrylamide is a popular strategy for the modification of lignocelluloses. Fan and co-workers employed laccase-initiated grafting using acrylamide and tertbutylhydroperoxide to functionalise jute fibres. ${ }^{[67]}$ Earlier studies confirmed that the presence of hydroperoxide was essential as the use of laccase only did not result in substantial grafting, while the necessity of laccase is dependent on the type of lignin. ${ }^{[68]}$ Modification resulted in a $10^{\circ}$ drop in SWCA and a somewhat enhanced colouration of the jute. These modest results indicate minimal grafting of acrylamide. Additionally, the initial SWCA of the jute proved to be exceptionally high $\left(110^{\circ}\right)$, which was likely to have been caused by contamination of the jute with fats and waxes. ${ }^{[26]}$ The presence of these compounds could also have influenced possible grafting, which makes these results hard to interpret. Ko and co-workers used a similar laccase-initiated grafting approach with $\mathrm{N}$-isopropylacrylamide (NIPAM) as monomer, but in a two-step sequence. ${ }^{[69]}$ Prior to grafting, they functionalised lignin with an ATRP-initiator ( $\alpha$-bromoisobutyryl bromide). Grafting subsequently resulted in 800 to $1000 \mathrm{~nm}$ long poly(NIPAM) brushes with thicknesses up to approximately $100 \mathrm{~nm}$ and polydispersity indexes as low as 1.3; which is common for brushes synthesised through ATRP. The downside of this type of polymerisation is inhibition by molecular oxygen. However, laccase requires oxygen as an electron acceptor; thus, exclusion of oxygen causes laccase not to act as a catalyst, but as a stoichiometric oxidant. Grafting of acrylamide, as mentioned previously, was not performed under oxygen-deprived conditions, which might also explain the modest functionalisation of jute.

Most of the work discussed so far had been conducted on laboratory scale, but laccase-mediated grafting has also been applied in more practical settings. Schubert and co-workers used industrial process water from a wood fibreboard plant directly as the phenol source in a laccase-mediated treatment to enhance the mechanical strength of wood fibre insulation boards. ${ }^{[70]}$ The compression strength and internal bond strength of boards made from laccase/process water-treated pulp were significantly improved relative to treatments with fresh water in the presence of laccase. This effect was enhanced even further when conventional latex binder was used as an additive. Pulp treated with a low laccase dose $\left(1.7 \mathrm{U} \mathrm{mg}^{-1}\right)$ and $3 \%$ latex, and pulp treated with $5 \%$ latex only, were equally strong; fossil-fuel-based binder dosage could thus be lowered by $40 \%$. For the production of mediumdensity fibreboards, Kharazipour applied a laccase/4-hydroxybenzoic acid treatment to replace traditional binders. ${ }^{[71]}$ By doing so, some grafting was likely to have occurred, but most pronounced was rapid delignification; the used lignin pulp was deprived of approximately $40 \%$ of its aromatics within $30 \mathrm{~min}$. This is remarkable as 4-hydroxybenzoic acid is a very unreactive mediator due to the presence of a conjugated carboxylic acid, while having only one ring-bound hydroxyl functionality. ${ }^{[72]}$ Nonetheless, fibreboards produced in this way complied with European standards regarding bending strength, internal bond strength and thickness swelling, whereas pulps treated with laccase only and 
two other laccase-mediator systems did not. Although a substantial contribution of adsorbed material can be expected, since in both studies the pulp has not been washed afterwards, this example does show the potential of laccase-assisted modifications in an industrial setting.

Aracri and co-workers used laccase-mediated grafting in a completely different, but applicationoriented, adhesive formulation. ${ }^{[73]}$ Several commercial isolated lignins were reacted in a laccase/mediator (acetosyringone) system to generate quinones, followed by grafting of gallic acid, tannic acid and dopamine, which is known to have strong adhesion properties. Further grafting at elevated temperatures generated phenol- and quinone-enriched lignin. The quinones in this paste could additionally undergo oligomerisation, nucleophilic attack, and/or imine bond formation when the paste was smeared at the backing of a wool carpet sample that naturally contained protein-based amine groups. In this way the paste could act as a carpet adhesive through both covalent and noncovalent interactions. The force required to pull a loop of fabric from the carpet was used to assess adhesion. The resulting adhesion was only approximately $10 \%$ lower than that of commercial latexbased adhesives, while being almost completely constructed from renewable sources.

To conclude: laccase-mediated grafting has proven to be a cost-effective, mild and eco-friendly way of modifying lignocelluloses. Whether grafting or rather adsorption is the main mode of material modification is highly dependent on process parameters and washing procedures. The latter requires extra attention in many cases, especially where non-covalent binding, and thus, leaching results in a health risk. Although Py-GC-MS and NMR spectroscopy are powerful techniques to study possible grafting, their use has not reached its full potential. Furthermore, the use of model compounds is essential in understanding the molecular details of the grafting process. It is, however, advisable to also employ both insoluble as well as non-phenolic lignin models in order to mimic lignin to a greater extent.

\section{Laccase-mediated grafting on polysaccharides}

To some degree, this topic has already been discussed, since applying laccase-mediator systems will sometimes also result in oxidation of the cellulose present in lignocelluloses. For some fibres, cellulose and hemicellulose are by far the most abundant constituents, e.g. $97 \%$ for flax fibres. ${ }^{[74]}$ For that reason, all cellulose considered herein is assumed to constitute less than $2 \%$ lignin.

Whereas the mechanism behind lignin modification is highly multifaceted due to its intrinsic complexity, laccase-mediated polysaccharide treatments are, in general, easier to comprehend. Two main modes of action could be considered dependent on the polysaccharide of choice: I) laccasemediator system-assisted oxidation of carbohydrate hydroxyl groups to aldehydes or carboxylic acids, followed by coupling with a suitable reagent, or II) laccase-mediated oxidation of a phenol that can be sequentially coupled to an amine-functionalised carbohydrate similar to the previously discussed functionalisation of cork with chitosan (Scheme 13). Even though laccase-assisted carbohydrate modification could be considered to be mechanistically less complex than that of lignin modification, the same guidelines apply and similar challenges remain. The intended grafting strategy should be mechanistically feasible and proper control experiments and washing should be conducted. Adsorption might also occur during carbohydrate modification so performing correct analysis and characterisation is sometimes puzzling.

In the mid-1990s Viikari and co-workers filed a patent describing, for the first time, the laccasemediated oxidation of cellulose in the presence of TEMPO. ${ }^{[75]}$ This strategy resulted in chemoselective oxidation of cellulose's C6 hydroxy groups. As mentioned earlier, laccase converts TEMPO through a one-electron oxidation to the corresponding oxoammonium ion that can selectively oxidise primary alcohols through a two-electron oxidation due to intrinsic steric bulk around the cation (Scheme 16). ${ }^{[76]}$ 


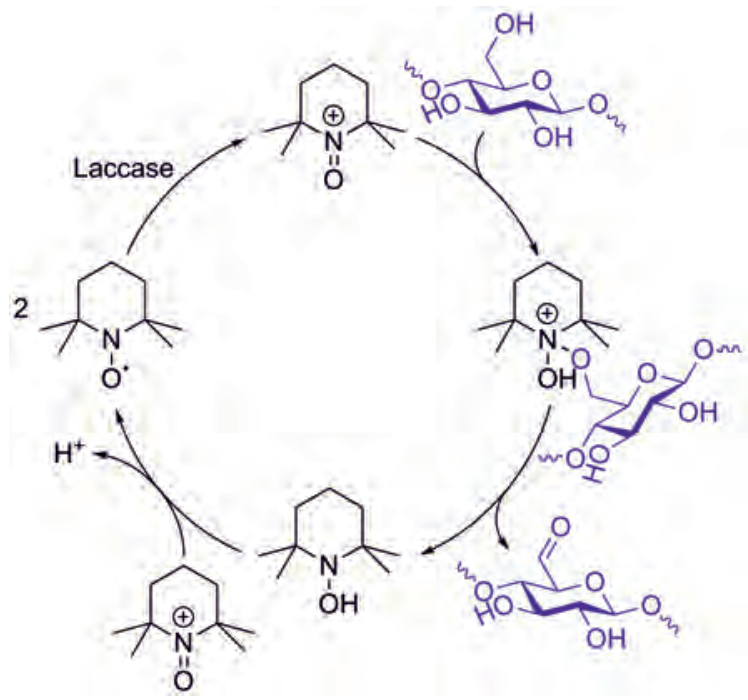

Scheme 16. Laccase-mediated oxidation of TEMPO followed by TEMPO-mediated oxidation of cellulose (based on the proposed mechanism of laccase/TEMPO-mediated oxidation by Tromp et al.). ${ }^{[76]}$

Where chemical oxidation of cellulose using bleach is known to cause depolymerisation of cellulose, laccase-mediated TEMPO treatments minimise this depolymerisation to a certain extent. ${ }^{[77]}$ The use of TEMPO as a mediator is essential, since hydroxylamine type mediators are not able to oxidise cellulose to a significant degree. Furthermore applying TEMPO without laccase does not result in substantial oxidation. ${ }^{[77]}$ Later studies showed that whether selectivity towards the aldehyde with respect to the carboxylic acid was observed was highly dependent on the conditions (Table 2). Evaluation of the carboxyl-to-carbonyl ratio resulting from oxidation of cellulose by $\mathrm{Xu}$ and coworkers ${ }^{[78]}$ and that of $\beta$-cyclodextrin (a cellulose model) by Yu et al. ${ }^{[79]}$ revealed a general trend that temperatures above $30{ }^{\circ} \mathrm{C}$, longer reaction times, higher laccase dosage and higher TEMPO concentrations favoured carboxylic acid formation. Apart from introducing additional functionality, paper made from oxidised cellulose is stronger, most markedly due to an increase in wet strength. ${ }^{[80]}$

Table 2. Variability in laccase/TEMPO-mediated cellulose oxidation: results from experiments with highest degree of oxidation.

\begin{tabular}{|c|c|c|c|c|c|c|c|}
\hline \multirow[b]{2}{*}{ Author } & \multirow[b]{2}{*}{$\begin{array}{l}\text { Laccase } \\
\text { source }\end{array}$} & \multirow[b]{2}{*}{ Type of pulp } & \multicolumn{4}{|c|}{ Concentration in $\mu \mathrm{mol} \mathrm{g}^{-1}$ [b] } & \multirow[b]{2}{*}{$\begin{aligned} & \mathrm{CO}_{2} \mathrm{H} \\
: & \mathrm{CHO}^{I \mathrm{c}}\end{aligned}$} \\
\hline & & & $\mathrm{CO}_{2} \mathrm{H}_{\mathrm{i}}$ & $\mathrm{CO}_{2} \mathrm{H}_{\mathrm{f}}$ & $\mathrm{CHO}_{\mathrm{i}}$ & $\mathrm{CHO}_{\mathrm{f}}$ & \\
\hline J iang ${ }^{[81]}$ & T. versicolor & $\begin{array}{c}\text { Bleached acacia Kraft pulp } \\
\text { (80\% cellulose) }\end{array}$ & 98 & 596 & 16 & 241 & $69: 31$ \\
\hline $\mathrm{Xu}{ }^{[78]}$ & A. oryzae & Bleached softwood Kraft pulp & 30 & 138 & 32 & 104 & $60: 40$ \\
\hline Aracri ${ }^{82]}$ & T. villosa & $\begin{array}{l}\text { Sisal soda-anthraquinone } \\
\text { ( } 79 \% \text { glucan, } 1 \% \text { lignin) }\end{array}$ & 110 & 266 & 1 & 191 & $45: 55$ \\
\hline Patel ${ }^{[77]}$ & T. pubescens & Cotton linters & 7 & 31 & 8 & 120 & $18: 82$ \\
\hline Aracri ${ }^{83]}$ & T. villosa & $\begin{array}{l}\text { Sisal soda-anthraquinone } \\
\text { ( } 79 \% \text { glucan, } 1 \% \text { lignin) }\end{array}$ & 110 & 126 & 1 & 107 & $13: 87$ \\
\hline J aušovec [84] & T. versicolor & Cellulose nanofibres (95\%) & 10 & 30 & 750 & 1523 & $3: 97$ \\
\hline
\end{tabular}

${ }^{[a]}$ First author and reference of corresponding paper. ${ }^{[b]}$ I nitial $\left(_{i}\right)$ and final $\left(_{f}\right)$ concentrations of carboxyl and carbonyl groups. ${ }^{\text {[c] }}$ Resulting carboxyl-to-carbonyl ratio.

Yu and co-workers used laccase/TEMPO-mediated oxidation in the presence of octadecylamine to increase the hydrophobicity of cotton. ${ }^{[85]}$ Initial modifications were performed on glucans (watersoluble short-chain glucose polymers), which are more reactive and easier to characterise. FTIR analysis of the modified glucan revealed (almost) full conversion of the aldehyde and the appearance of a new band at $\bar{v}=1640 \mathrm{~cm}^{-1}$, which corresponded to the formed imine. However, due to strong 
absorbance of cotton at that wavelength, no increased intensity of the band at $\bar{v}=1640 \mathrm{~cm}^{-1}$ was observed after cotton modification. Nonetheless, SWCA measurements revealed that cellulose was rendered hydrophobic (SWCA $>110^{\circ}$ ). Washing fastness was high, since the SWCA was barely altered for up to eight washing cycles, even though washing was performed in the presence of a commercial detergent.

The importance of washing steps, a proper understanding of adsorption/grafting mechanisms and appropriate analytical tools was nicely accentuated by Guimarães and co-workers, who overdyed denim fabrics with in-situ laccase-generated catechin or catechol oligomers. ${ }^{[86]}$ Dyeing in this case, does not result in covalent grafting due to the lack of reactivity between cellulose and (oxidised/oligomerised) catechin or catechol. Nonetheless intense colouration of the fabrics with sufficient to good fastness levels was achieved. It was hypothesised that less soluble oligomers had reasonably strong interactions with the denim surface. This thus indicates that non-specific adhesion can be very resilient. Even when grafting is possible, mere adsorption cannot always be ignored.

Where Py-GC-MS analysis proves to be very promising in the field of lignocellulosics, Díaz Blanco and co-workers envisioned an elegant coupling of breakdown, LC-MS-MS and NMR spectroscopy to fulfil a similar role in the analysis of polysaccharide grafts. In their approach cellulose dyeing was achieved by firstly tosylating cellulose, followed by nucleophilic displacement of the tosylate with 2,5diaminobenzenesulfonic acid (2,5-DABSA). ${ }^{[87]}$ Further functionalisation was thereafter accomplished through laccase-mediated grafting from the immobilised 2,5-DABSA using catechol. Removal of unbound catechol was achieved by extensive washing of the modified cellulose in boiling water in the presence of a surfactant. Grafting was indirectly confirmed by showing that aminated cellulose dyed with ex-situ generated oligomeric catechol exhibited a far lower wet rubbing fastness. MS-MS analysis of a modified cellulose hydrolysate revealed the presence of glucose dimers covalently attached to one molecule of 2,5-DABSA, which bound two to six units of catechol (Scheme 17). Similar dimers were found where a tosylate was still present, indicating that nucleophilic displacement by 2,5-DABSA was not complete. Breakdown of the graft followed by analysis thus proves to also be a powerful tool for studying grafting on cellulose.

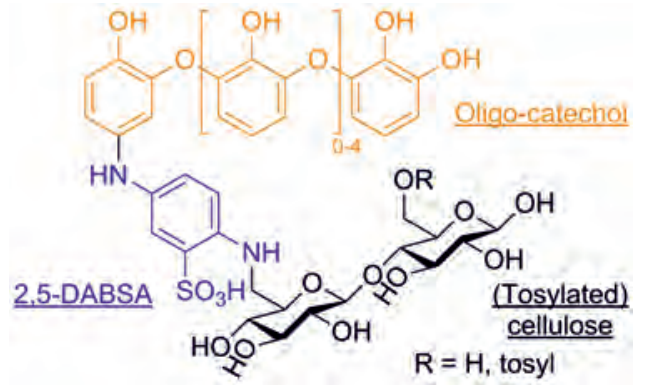

Scheme 17. Possible covalently bound structures of 2,5-DABSA/catechol-modified cellulose observed by MS (as proposed by Díaz Blanco and co-workers). ${ }^{[87]}$

For many grafting applications the outermost layer of the material should be activated, but its internal consistency should not be altered. Because TEMPO-mediated oxidation of cellulose decreases the degree of polymerisation, Liu and co-workers developed an immobilised TEMPO-poly(vinylamine) complex (PVAm-T) and used this as mediator in the laccase-mediated oxidation of cellulose. ${ }^{\left[{ }^{[8]} \text { It was }\right.}$ proposed that some surface C6 hydroxy groups got oxidised to the aldehyde first, which would then form imine bonds with the remaining amine groups of PVAm-T (Scheme 18). Since PVAm-T is still positively charged and laccase is slightly negatively charged, they formed a PVAm-T/laccase complex on the surface. It was further hypothesised that this immobilised laccase would oxidise proximal TEMPO units to their corresponding oxoammonium ions. Since oxidation was only observed at the exterior of the cellulose membranes, it was assumed that TEMPO did not act as a direct shuttle for oxidising cellulose, but that it oxidised neighbouring TEMPO units on PVAm-T. These neighbouring 
TEMPO units would do the same until the TEMPO moiety closest to the surface would oxidise the C6 primary alcohol of cellulose. Since only the exterior of cellulose is oxidised (Scheme 18B), lower immobilised TEMPO concentrations (with respect to those of free TEMPO) were required for oxidation.

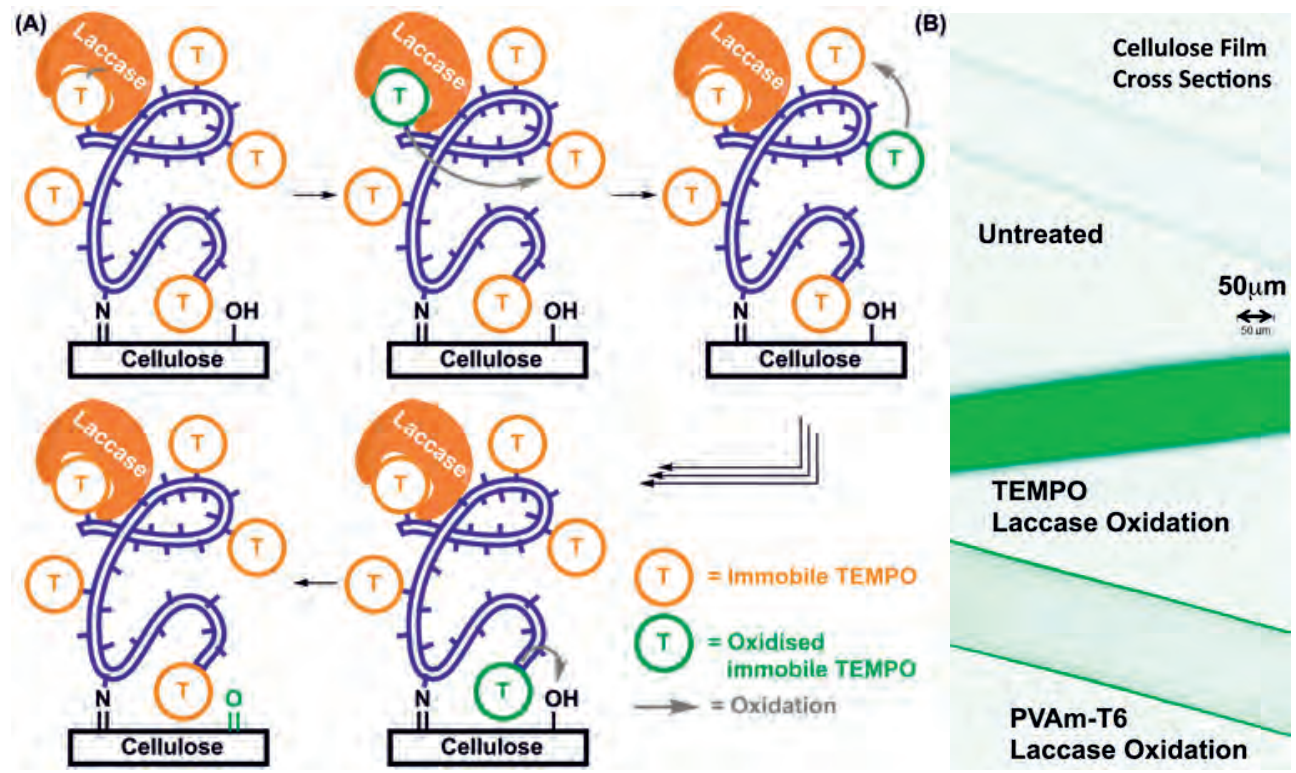

Scheme 18. A: PVAm-T/laccase complex grafted on cellulose for the laccase-mediated oxidation of TEMPO followed by "oxidation state-transfer" to oxidise cellulose (based on Liu et al.). ${ }^{[88]}$ B: Distribution of fluorescein-labelled aldehyde groups in cellulose membrane cross sections. Aldehydes were labelled with green fluorescein-5thiosemicarbazide. Reproduced with permission from Liu et al.). ${ }^{[88]}$ Copyright American Chemical Society, 2013.

Furthermore, the PVAm-T/laccase complex was used, together with non-functionalised poly(vinylamine), as a cellulose-to-cellulose adhesive. In later studies, the use of poly(acrylic acid)TEMPO instead of PVAm-T as the source of TEMPO did not result in adhesion. ${ }^{[89]}$ Adhesion could be restored by adding poly(vinylamine) again, thereby indirectly indicating the importance of grafting.

Similar to the hydrophobisation of lignocelluloses, cellulosic material can also be modified using laccase and dodecyl gallate. ${ }^{[90]}$ These celluloses have low lignin $(\sim 1 \%$; kappa number $<6.3)$ and hexenuronic acid contents, which make grafting unlikely. Nonetheless increases in kappa number, although limited, are observed after laccase-mediated modification and extraction with acetone. This means that either the graft consists of long gallate oligomers, or acetone extraction is simply not sufficient to remove all unbound gallate and gallate oligomers.

In order to create a conductive form of cellulose, Zhang and co-workers performed in-situ laccasemediated synthesis of polyaniline to generate highly conductive cotton which displayed enhanced antistatic properties. ${ }^{[91]}$ By using sodium dodecylbenzenesulfonate as a micellar soft template, polyaniline was synthesised in a linear head-to-tail fashion (Scheme 19). Although covalent bonding to cellulose was not claimed, some imine formation might have taken place. This will, however, be limited as laccase's ability to oxidise cellulose to aldehydes is minor when TEMPO is not applied as mediator.

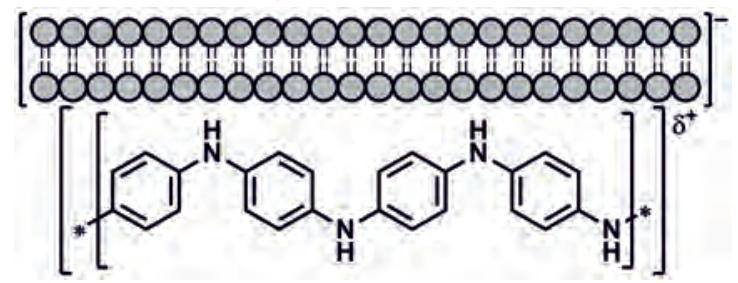

Scheme 19. Micellar soft template-assisted head-to-tail synthesis of polyaniline. 
In more recent work, sodium dodecylbenzenesulfonate was replaced by potassium perfluorooctanesulfonate (PFOS), which provided cellulose with the possibility to perform surface energy switching, and thus, also wettability switching (Scheme 20). ${ }^{[92]}$ In this elegant approach, the functionalised surface displayed SWCA $>120^{\circ}$ due to the presence of PFOS, which not only acted as a template, but also as a dopant. Dedoping could subsequently be achieved by using ammonia or ethylenediamine to result in a fully wettable $\left(\right.$ SWCA $<15^{\circ}$ ) surface; this process was repeatable up to more than ten times. Concomitantly, the cellulose appeared blue/purple in the dedoped state (green when PFOS-doped) and was no longer conductive. The equivalent of 25 repetitive water washing cycles resulted in a $30^{\circ}$ decrease of the SWCA in the doped state. Although this is substantial, the SWCA is still far higher than that of bare cellulose, indicating that polyaniline and/or PFOS is still present on the surface, which could point to covalent bonding between polyaniline and cellulose.
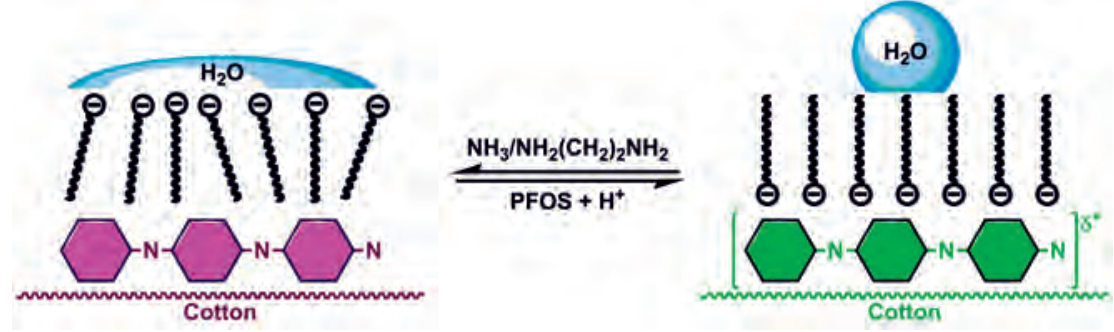

Scheme 20. Wettability switching of polyaniline-functionalised cotton due to doping with PFOS and dedoping with amines, including a schematic depiction of a water droplet deposited on the respective surface. ${ }^{\text {[92] }}$

Apart from cellulose itself, its etherified derivative, ethyl cellulose, has also been used in laccasemediated transformations. I qbal and co-workers used several approaches towards the development of ethyl cellulose composites. By mixing ethyl cellulose and keratin in the presence of laccase, composites with SWCA's and glass transition temperatures higher than those of either parent material were developed. ${ }^{[93]}$ The exact role of laccase is, however, debatable; since most of the hydroxy functionalities of the cellulose backbone are etherified and no mediator is applied, oxidation of cellulose towards reactive aldehydes is unlikely. The authors proposed amide formation via oxidation of the scarce hydroxy moieties of ethyl cellulose which couple to amine groups of keratin. However, to the best of our knowledge, this type of laccase-assisted amide-bond formation has not been observed before. In a later study, this material was further functionalised through a laccase-mediated approach involving several simple phenols. ${ }^{[94]}$ Cellulose-keratin-based composites treated in the presence of caffeic acid, gallic acid, thymol or 4-hydroxybenzoic acid showed complete bacteriostatic activity towards the gram-negative strains $E$. coli and $P$. aeruginosa. Furthermore, similar activity was observed for thymol and 4-hydroxybenzoic acid-modified composites against B. subtilis and S. aureus. Additionally, human keratinocyte-like (HaCaT) skin cells proved to be fully viable in the presence of these composites after five days of growth, while maintaining properly stretched morphologies. Grafting most likely occurred through $\mathrm{C}-\mathrm{N}$ or $\mathrm{C}=\mathrm{N}$ bond formation between the amine moieties of keratin and oxidised phenols. However, a substantial contribution of adsorbed phenolic oligomers can also be expected, since the composites were washed only after being dried. Drying of the swollen ethyl cellulose will cause compression of the material. During compression, deposition of unbound phenolics, that are still present, is likely to occur. This will impede their removal through washing afterwards. Nonetheless, the phenol-functionalised ethyl cellulose/keratin composites might prove to be easily accessible materials for several biotechnological applications.

The same authors used a similar approach to develop ethyl cellulose/poly(3-hydroxybutyrate) composites. ${ }^{[95]}$ Again composites that were generated in the presence of laccase exhibited higher glass transition temperatures than either of their parent compounds. Although claimed, laccase-mediated grafting is unlikely due to the absence of a mediator and a deficiency in (nucleophilic) coupling partners in poly(3-hydroxybutyrate) $(\mathrm{P}(3-\mathrm{HB})$ ). Nonetheless, composites generated in the absence of 
laccase did not show an increase in glass transition temperature. Incorporation of laccase itself within the composite might be a reason for this. Further functionalisation of these materials was achieved by modification with either 4-HBA or FA in the presence of laccase. ${ }^{[96]}$ Both modifications resulted in surfaces with bacteriostatic activity against Escherichia coli. Of these, 4-HBA-functionalised ethyl cellulose/P(3-HB) proved to be biocompatible with HaCaT cells. Any property enhancement has likely occurred due to deposition of phenolic oligomers because there is no basis for reactivity between (oxidised) phenolics and ethyl cellulose or $\mathrm{P}(3-\mathrm{HB})$. Similar results were found when thymol, caffeic acid or gallic acid were used as the phenolic modifier, although caffeic acid and gallic acid modification did not result in equally efficient anti-bacterial composites. ${ }^{[97]}$ Iqbal and co-workers also employed lipase instead of laccase to generate composites from ethyl cellulose and $\mathrm{P}(3-\mathrm{HB})$ through esterification. ${ }^{[98]}$ Although ethyl cellulose possesses a limited number of hydroxy moieties, this seems a more viable way of covalently coupling ethyl cellulose and $\mathrm{P}(3-\mathrm{HB})$.

Of all amine-bearing carbohydrates, chitosan (CS) is most commonly employed in grafting. CS

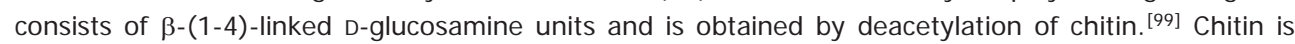
the second-most abundant natural carbohydrate polymer, but generally regarded as waste; thus, recycling it as CS is highly desirable. The solubility of CS is strongly dependent on pH; at a pH lower than 6.1, enough amine groups are protonated to dissolve CS. ${ }^{[100]}$ For the sake of consistency, we will mainly discuss heterogeneous reactions with insoluble CS.

Božič and co-workers and Aljawish et al. (vide infra) more or less simultaneously pioneered the field of laccase-mediated modification of chitosan. The former initially modified CS by functionalising it with caffeic acid and gallic acid oligomers to confer anti-oxidant and anti-microbial properties to the material.. ${ }^{[101]}$ By using Trametes versicolor laccase, chitosan was modified at different acidities. Of these, only laccase/gallic acid treated $\mathrm{CS}$ at $\mathrm{pH} 4.5$ showed improved anti-bacterial activity (against $E$. coli) relative to $\mathrm{CS}$ treated at that $\mathrm{pH}$ without the presence of laccase. ABTS radical cation scavenging activity, a measure of the anti-oxidant activity, was excellent for CS derivatives, modified with either of the phenolics at all acidities. Due to limited washing of the resulting grafts, the degree of actual covalent grafting is hard to determine. Laccase-mediated treatments with other phenolics (quercetin and tannic acid) to modify CS resulted in no or limited increases in anti-microbial activity. ${ }^{[02]}$ By employing Suberase, a Myceliophthora thermophila laccase, Aljawish et al. modified CS with either FA or ethyl ferulate under heterogeneous reaction conditions followed by extensive washing with buffer, but also with methanol, ethanol and acetone, to remove moderately soluble oligomers. ${ }^{[103]}$ Similarly to the above-mentioned CS derivatives, a pronounced anti-oxidant effect was observed, although FAmodified $\mathrm{CS}$ exhibited $\mathrm{EC}_{50}$ values that were approximately three times higher than those of the ethyl ferulate-modified derivatives. The extensive washing procedure allowed for better characterisation of the CS derivatives. FTIR analysis revealed two new bands at $\bar{v}=1620$ and $1640 \mathrm{~cm}^{-1}$ for both grafted phenolics. The presence of these two bands might indicate covalent grafting, since these could correspond to $\mathrm{C}=\mathrm{N}$ stretching vibrations of Schiff bases. Although only speculative, additional evidence for the presence of Schiff bases might be found in partially overlooked, but valuable, ${ }^{13} \mathrm{C}$ NMR spectra of derivatised CS. Any imines present would be formed from a reaction between an amine and a quinone. The peak at approximately $150 \mathrm{ppm}$ could represent the carbon atom of a quinone-based imine. ${ }^{[104]}$ This is further substantiated by the absence of this signal in the spectrum of hydrated CS derivatives, since the imine would be in equilibrium with its parent amine and quinone. It was furthermore shown that bacterial growth on both ethyl ferulate and FA-modified CS was comparable to that of unmodified chitosan. By combining these results with those of Božič and co-workers, it could thus be concluded that laccase-mediated functionalisation using simple phenols is not a viable way to improve the anti-bacterial behaviour of CS. Modification additionally decreased water and oxygen permeability of the material, which indicated its potential as (food) packaging material. ${ }^{[105]}$ Due to its increased hydrophobicity, modified CS adsorbed more protein from cell growth media than CS itself did, which is essential for the viability of cells. ${ }^{[106]}$ Cell growth of human umbilical vein endothelial cells (HUVEC's) and mesenchymal stem cells was improved and mainly influenced by grafting time and 
(derivatised) CS film thickness. An approximate $20-40 \%$ increase in cell viability was achieved relative to that of non-modified CS, while also improving cell morphology from round to stretched.

Yang and co-workers employed the methodology established by Aljawish et al.; ${ }^{[103]}$ thus including extensive washing, to modify CS with cinnamic acid derivatives to minimise bacterial growth of several Ralstonia solanacearum races. ${ }^{[107]}$ Of main interest was the growth inhibition of RS-5, which is responsible for mulberry wilt disease. Of all functionalised chitosan derivatives, the caffeic acidmodified chitosan proved to be the most promising with an IC 50 value of $0.23 \mathrm{mg} \mathrm{ml}^{-1}$, which was similar to that of the oligomerised caffeic acid itself.

In summary, laccase-mediated covalent polysaccharide functionalisation is a growing field that allows easy and eco-friendly access to highly complex and functional materials. Promising biotechnological applications, such as anti-bacterial and biodegradable plastics that enhance cell growth, rapidly emerge. However, the chemistry behind this technology has thus far not been substantially studied. Many of the developed procedures for laccase-mediated grafting lack proper washing cycles, which makes differentiation between adsorption and covalent grafting challenging. Studies with polysaccharide model compounds, such as simple glucoses and glucosamines, might aid in establishing a better understanding of the underlying chemistry. Furthermore, most of these modifications are based on imine-bond formation, which is reversible in nature. For numerous applications, this is not desirable, and chemical or enzymatic reduction of the imine bond would introduce a more stable graft. Overall, it is therefore a fair characterisation of the state-of-the-art in this field to describe laccase-mediated grafting on lignocelluloses as promising, but also that there still is a lot to be learned.

\section{Laccase-mediated grafting on proteins}

Apart from grafting on textiles such as jute and cotton, laccase is also commonly employed to functionalise wool fibres. The high abundance of nucleophiles in wool keratin allows covalent bonding to phenols oxidised by enzymes. ${ }^{[108]}$ Laccase-mediated grafting is regularly used for in-situ colouration, enhancement of hydrophobicity or to confer anti-bacterial properties to the material. An important factor in wool modification is prior extraction and cleaning because raw wool is often contaminated with feculence and is abundant in fats and waxes. ${ }^{[109]}$

Jeon and co-workers studied laccase-mediated in-situ colouration of yak wool with a comprehensive set of phenols and phenolic mixtures. ${ }^{[110]}$ Strongly coloured oligomers that covered a wide range of tints were synthesised firstly. From this, mixtures displaying colours that resembled those of natural human hair were selected and further tested for their dyeing ability. Modification with gallic acid and syringic acid resulted in brown wool, catechol and catechin gave black wool, and FA/syringic acid modification resulted in a faint reddish colour (Figure 1). Washing with detergents commonly encountered in shampoo did not result in loss of colour. Although this was not elaborated on, the good washing fastness might have resulted from covalent binding between keratin and the oxidised mono- or oligomers, although the poor solubility of phenolic oligomers might also have played a role.

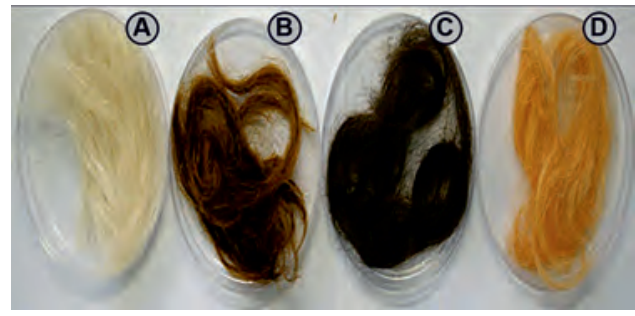

Figure 1. A: Virgin yak wool, B: yak wool dyed with gallic acid plus syringic acid, C: yak wool dyed with catechin plus catechol and D: yak wool dyed with ferulic acid plus syringic acid (reproduced with permission from Jeon and coworkers). ${ }^{[110]}$ 
Yuan and co-workers used a similar approach to colour wool with catechin or gallic acid (Scheme 21A). ${ }^{[111]}$ To also impede shrink resistance to the fabric, a polymer coating can be applied to smoothen the wool cuticle surface. The addition of poly(ethyleneimine) (PEI) to the laccase/phenol mixture in a one-step process, however, prevented colouration of the fabric (Scheme 21B).

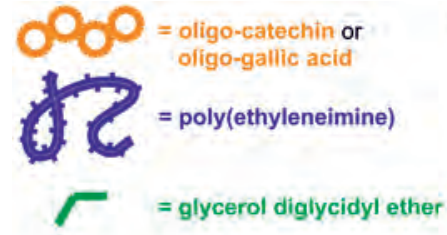

(C)
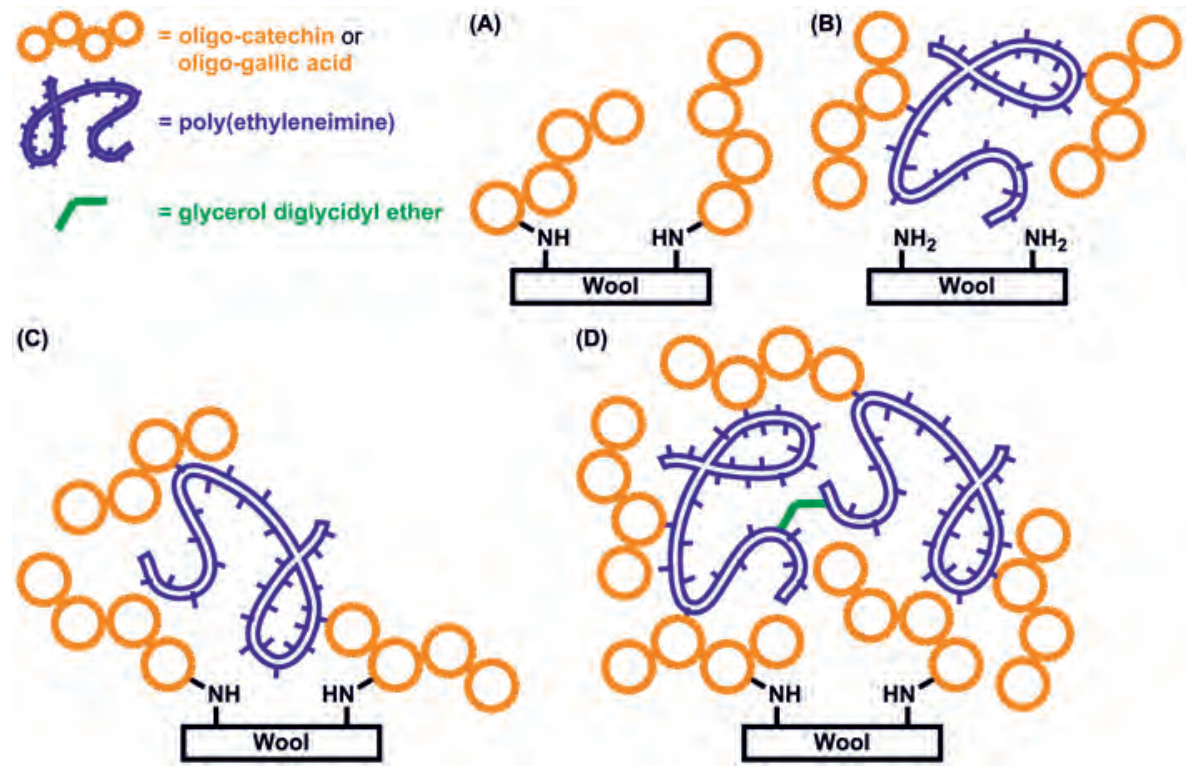

Scheme 21. Schematic representation of laccase-mediated colouration of wool with catechin or gallic acid oligomers. A: Colouration of wool with laccase-generated oligomers only. B: One-step attempt at colouration in the presence of poly(ethyleneimine). C: Two-step process: colouration followed by the addition of poly(ethyleneimine). D: Two-step process: colouration followed by the addition of poly(ethyleneimine) and glycerol diglycidyl ether (based on Yuan et al.). ${ }^{[111]}$

It was hypothesised that the amine groups of PEI competed with nucleophilic moieties of wool for covalent binding to the oxidised phenols. Indeed, implementation of a two-step/one-pot process, in which laccase-mediated modification was followed by the addition of PEI did result in successful colouration and an average 15\% increase in shrink resistance (of alkali pre-treated samples) after four washing cycles (Scheme 21C). Furthermore, similar levels of colouration in the one- (without PEI) and two-step processes imply that colouration has substantially occurred through covalent grafting, since otherwise PEI would also have been covalently bound to loosely adsorbed phenolics in the two-step process. Covalent binding is further suggested by the fact that the fabrics remain equally strongly coloured after four washing cycles. Shrink resistance could further be increased by the addition of amine crosslinking agent glycerol diglycidyl ether in the second step to increase PEI polymer length. Addition of the crosslinker also further increased the colour strength of the fabric, most likely because dissolved coloured PEI could be crosslinked to the surface-immobilised amines (Scheme 21D).

Other work by the same group involved the in-situ laccase-mediated synthesis of poly(2,5diaminobenzenesulfonic acid) (poly(2,5-DABSA)) to confer pH-dependent colouration and conductivity to wool. ${ }^{[112]}$ Poly(2,5-DABSA)-coated wool doped at $\mathrm{pH} 1.8$ was conductive and dark purple, while after dedoping at $\mathrm{pH}$ 10, the modified wool was rendered electrochemically inactive and became yellowish/brown. Although no covalent bonding between wool and the coating was claimed, this is likely to have occurred as 2,5-DABSA could also be oxidised by laccase to generate a radical, which process is similar to that of radical generation from phenols. This makes this moiety thus also susceptible to attack by amines of the wool protein.

To enhance laccase-mediated colouration of wool even further, Zhang and co-workers pre-treated wool with 1-ethyl-3-(3-dimethylaminopropyl)carbodiimide hydrochloride (EDC) to activate carboxylic 
acids present on wool.[113] Next to direct coupling of wool to oxidised phenolics, phenols could now bind to wool through ester-bond formation. This methodology indeed resulted in a $50 \%$ colour strength increase relative to wool that had not been pre-treated. Additionally, substantial improvements in wet rubbing fastness and washing fastness were achieved. In order to further examine the degree to which adsorbed oligomers contribute to colouration, washing with more apolar solvents would be desirable.

Hossain and co-workers employed laccase-mediated oligomerisation of nordihydroguaiaretic acid (Scheme 22) for the functionalisation of wool fibres. ${ }^{[114]}$ Modification resulted in an increased UV resistance, improved anti-oxidant activity, a $25 \%$ tensile strength increase and better shrink resistance. The improvement of the latter two physical parameters was ascribed to crosslinking of fibres due to the bi-catechol moiety of nordihydroguaiaretic acid. Unfortunately, no distinctive characteristics of covalent bonding, such as imine formation, were reported.

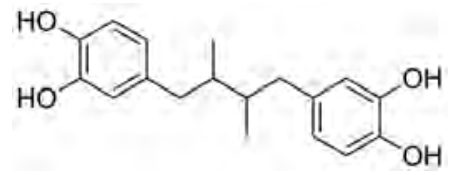

Scheme 22. Nordihydroguaiaretic acid.

As with cotton and lignocellulosic fibres, dodecyl gallate has the potential to also graft on wool through radical coupling, Michael addition or Schiff base formation. By doing so, wool was made hydrophobic (SWCA $>110^{\circ}$ ), while, at the same time, conferring upon it moderate anti-oxidant and anti-bacterial properties. ${ }^{[115]}$ Additional washing with aqueous solutions of ethanol and at higher temperature most likely assured proper removal of any adsorbed material. It was claimed that laccasemediated modifications with higher concentrations ( $>1 \mathrm{mM}$ ) did, however, result in substantial adsorption. Further studies revealed that laccase-mediated modifications with gallates bearing shorter alkyl chains were less efficient at conferring anti-bacterial activity, anti-oxidant activity and hydrophobicity to the wool. ${ }^{[116]}$

By laccase/TEMPO-mediated oxidation of $\beta$-cyclodextrin, wool could be functionalised with this cyclic heptasugar through imine-bond formation. ${ }^{[79]}$ MALDI-TOF MS analysis revealed that, in initial tests with tyrosine as a wool model compound, all seven sugar moieties in one molecule of $\beta$ cyclodextrin could be simultaneously functionalised. Wool functionalisation was claimed to be confirmed by an increase in the $\mathrm{C}-\mathrm{O}, \mathrm{O}-\mathrm{H}$ and $\mathrm{N}-\mathrm{H}$ stretching bands in FTIR. Furthermore, a new band at $\bar{v}=1502 \mathrm{~cm}^{-1}$ was attributed to the $\mathrm{C}=\mathrm{N}$ stretching vibration of newly formed imines. However, it is well-known that imine formation results in a new band between $\bar{v}=1600$ and $1700 \mathrm{~cm}^{-1}$. $\left.{ }^{\text {85, }}, 103,117\right]$ Although likely, imine formation following cellulose modification could thus not be unambiguously confirmed.

Mussel-inspired modification of surfaces is a hot topic because dopamine derivatives provide easily applicable and highly adhesive coatings. ${ }^{[118]}$ Oxidation of dopamine furthermore results in melanin polymers that provide resistance against UV light by absorbing it and dissipating it as heat. J ia and coworkers used laccase to oxidise dopamine and make silk UV resistant. ${ }^{[119]}$ Polydopamine coatings were applied in two ways: I) by first adsorbing dopamine on the silk surface, followed by dipping the silk in a laccase solution, or II) through in-situ laccase-mediated dopamine polymerisation in the presence of silk. Although method two provided higher colour strength, washing fastness was inferior. Further washing was attempted by treating the samples with dimethylformamide at $90^{\circ} \mathrm{C}$. The negligible drop in colour strength for silk modified with either method was ascribed to result from covalent binding of melanin to silk. Interestingly, XPS analysis revealed an increase in surface nitrogen from $3 \%$ for unmodified silk to $19 \%$ for polydopamine-modified silk. Elemental nitrogen only accounts for approximately $9-10 \%$ of all elements in melanin, any increase above that would thus indicate the presence of other substances, for example laccase. However, it is more likely that surface nitrogen is more abundant in unmodified silk than initially indicated. ${ }^{[120]}$ 
To conclude: much of the work presented above focused on applying laccase-mediated strategies to modify wool. However, only limited fundamental knowledge on the grafting mechanism has been gained since the first laccase-mediated dyeing process was developed by Shin and co-workers. ${ }^{[121]}$ In order to properly identify whether actual grafting plays a role, the functionalisation mechanisms should be investigated. Questions such as "does Schiff base formation occur?" and "do amino acid thiols perform nucleophilic attack on laccase oxidised phenols?" need to be answered. Furthermore, to this point, the scarcity in studies performing proper washing and control experiments hamper further discrimination between grafting and adsorption. Nonetheless, laccase-mediated functionalisation proves to be, among others, an easily applicable and eco-friendly way to colour wool fabrics.

\section{Laccase-mediated grafting on synthetic polymers}

Functionalising synthetic polymers forms a unique sub-domain in the field of laccase-mediated grafting, since these types of modifications are not based on a common grafting mechanism. To overcome the inherent inertness of polypropylene, Schroeder and co-workers used plasma-initiated radical polymerisation in the presence of an amine-functionalised methacrylate monomer. ${ }^{[122]}$ The amine-functionalised co-polymer was subsequently used in laccase-mediated grafting of guaiacol sulfonic acid. Laccase-mediated grafting efficiency was directly related to the type of aminefunctionalised methacrylate present on the surface; only primary amines reacted with the oxidised phenolics, where tertiary amines and quaternary amines did not. MS analysis of the laccase-mediated coupling of guaiacol sulfonic acid and hexylamine (an amine-functionalised polypropylene model compound) revealed the presence of a signal at $m / z$ 301.7. This signal was attributed to hexylamine coupled to guaiacol sulfonic acid, thereby presenting a basis for the possible grafting of the phenolic monomer on the functionalised polymer.

Díaz Blanco and co-workers also employed oxygen plasma to hydroxylate flat poly(dimethylsiloxane) (PDMS, Scheme 23). ${ }^{[123]}$ The hydroxylated surface was then treated with (3aminopropyl)-triethoxysilane (APTES) to generate amine-functionalised PDMS (PDMS- $\mathrm{NH}_{2}$ ). A two-step laccase-assisted grafting strategy was thereafter employed to couple gallic acid oligomers to PDMS$\mathrm{NH}_{2}$, followed by further modification with sulfobetaine methacrylate (SBMA).

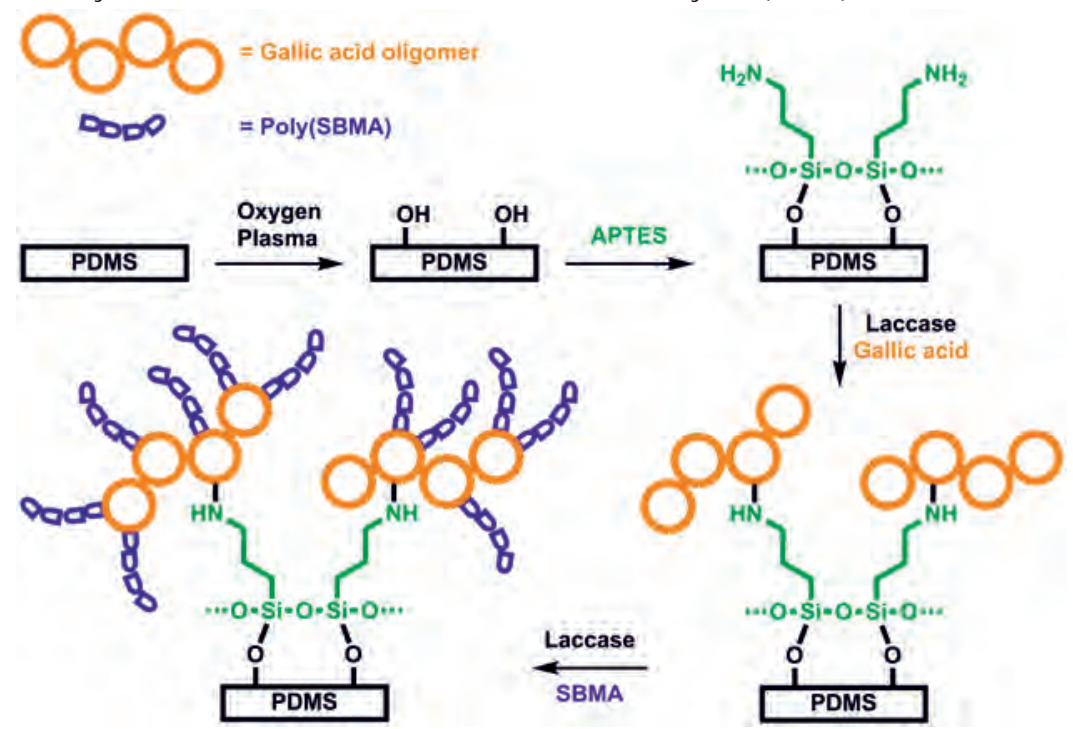

Scheme 23. Schematic representation of laccase-mediated SBMA functionalisation of PDMS (based on Díaz Blanco et al. $\left.{ }^{[123]}\right)$. 
XPS analysis allowed for the discrimination between several types of chemical species on the surface. In this way, the presence of $\mathrm{sp}^{2}$-hybridised nitrogen and quaternary nitrogen could be proven with reasonable reliability. Unfortunately, this is one of the few studies regarding laccase-mediated grafting that harnesses the power of high-resolution XPS narrow scans; many other grafting studies would greatly benefit from its use. This functionalisation strategy (silanisation, followed by laccasemediated grafting) was also applied to PDMS-based urinary catheters (PDMS-SBMA). I nitially, PDMSSBMA and SBMA-coated catheters that did not undergo plasma treatment (PDMS-SBMA ) exhibited similarly good anti-fouling properties against synthetic urine. However, fouling was still only minimal for PDMS-SBMA after seven days of incubation, while PDMS-SBMA ${ }^{x}$ was severely fouled in that time frame. Furthermore, the coating proved to only induce a minimal reduction in human foreskin fibroblast viability $(\sim 10 \%)$ compared with cells grown only in medium. Static growth of $P$. aeruginosa and S. aureus on PDMS-SBMA was reduced by approximately $60 \%$, relative to growth on PDMS. However, in a dynamic setup with an artificial bladder, this effect proved to be less pronounced.

Gonçalves and co-workers utilised a simpler strategy in order to functionalise catheters. Their approach involved an alkaline pre-treatment of polyurethane and PDMS catheters followed by laccasemediated modification with catechin. ${ }^{[124]}$ Modification of both materials, either with or without pretreatment, led to coating of the surface, as indicated by an increase in colour strength. Furthermore, pre-treated samples exhibited higher colour strength than those that did not undergo pre-treatment. This could be rationalised by appreciating that alkaline pre-treatment hydrolysed some of the urethane moieties to expose amines that could covalently bind to oxidised catechin. Water washing did not result in a substantial reduction of colour strength; however, washing with a detergent in all cases resulted in a drop in colour strength. This caused the colour strength of samples that were alkaline pre-treated not to be any different to those of samples that were not pre-treated. Since, without pretreatment, only a limited number of amines are able to covalently bind catechin (oligomers), this indicates that a substantial part of the colouration is caused by mere deposition of catechin oligomers. In the case of silicone catheters, a considerable loss of colour was subsequently observed after incubation in synthetic urine. Nonetheless, substantial anti-microbial activity against $E$. coli was observed in many cases and in some cases against $S$. epidermidis.

To graft ferulic acid from polyamides, Acero et al. employed a two-step enzymatic cascade.[125] Polyamidase was first used to partially hydrolyse the polyamide to expose amines that, in the second step, covalently bound to laccase-oxidised FA. The use of butylamine as a model compound in a laccase-mediated coupling with FA resulted in a 1:1 covalently bound conjugate between FA and the amine, as analysed by MS. This result makes grafting likely, but more in-depth analysis of the graft is required.

Fouling is not only an issue that concerns medical devices, but it also induces problems in the shipping and water filtration industries, for example. ${ }^{[126]}$ Water filtration membranes are made out of inert polymers to reduce chemical deterioration. ${ }^{[127]}$ However, chemically inert membranes tend to be hydrophobic, which induces biofouling through protein adsorption followed by biofilm formation. Previous work within our group by Nady and co-workers, involved the modification of inert poly(ethersulfone) (PES) water filtration membranes through laccase-mediated coating with simple phenolics. ${ }^{[128]}$ To improve hydrophilicity of the membrane, polar hydroxybenzoic acids, such as 4hydroxybenzoic acid (4-HBA) and gallic acid, were employed as phenol source. Subsequent colouration of the membranes indicated coating. To test whether, and to what extent, this change was caused by adsorption, the membranes were also incubated with a solution of phenolic oligomers which had been generated ex-situ and in which laccase was deactivated by using dilute sodium hydroxide. It was observed that in the case of gallic acid, adsorption accounted for more than $87 \%$ of the colour change. The colouration of membranes caused by pre-oligomerised 4-HBA, however, diminished by only approximately $35 \%$ compared with colouration induced through in-situ laccase-mediated oligomerisation. It was therefore assumed that covalent grafting from the membrane took place in the case of 4-HBA. By employing quantum chemical calculations, it was made probable that the graft 
consisted of both C-O- and C-C-coupled oligomers (Scheme 24A). Later studies confirmed this C-O and $\mathrm{C}-\mathrm{C}$ bond formation and it was further observed that the dimers that were initially formed, rapidly oligomerised onwards; thus indicating that the membrane coating was likely to be composed of highly diverse and relatively long oligomers. ${ }^{\text {72] }}$

(A)
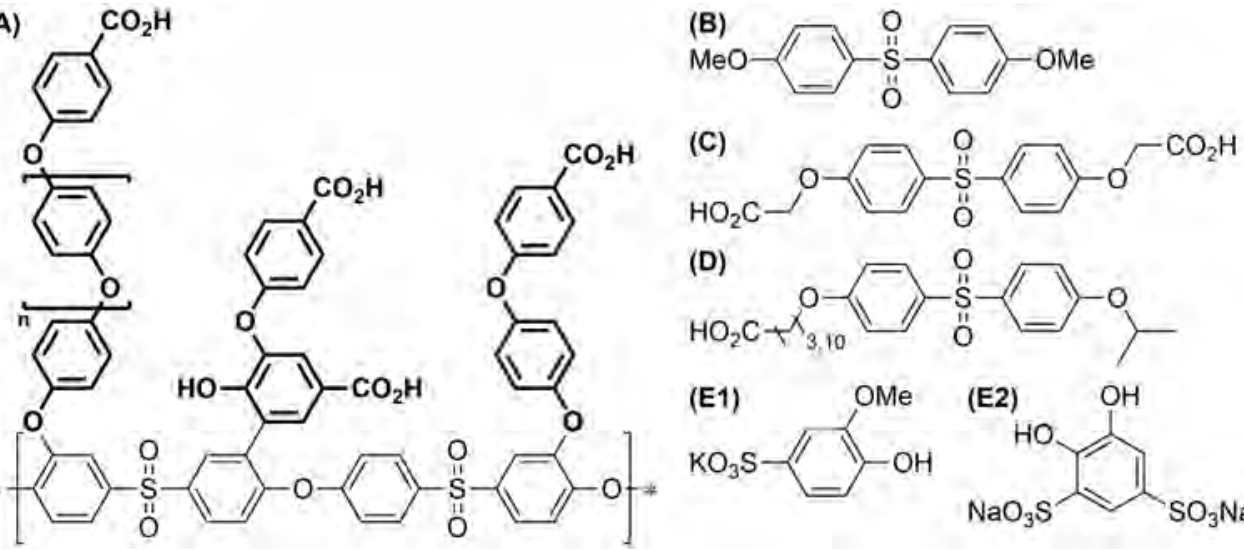

(D)
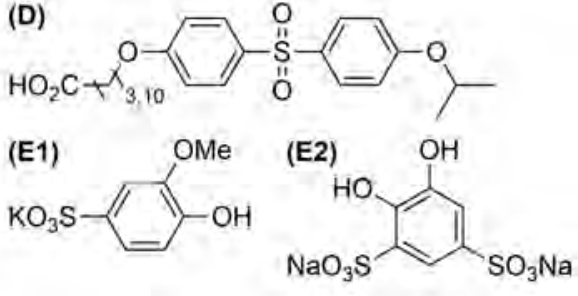

Scheme 24. A: Result of laccase-mediated grafting from PES membranes, as proposed by Nady and co-workers. ${ }^{[128]}$ Compounds used in the discrimination between grafting and adsorption on PES membranes. B: Insoluble PES model compound, C: water-soluble PES model compound and D: PES model compound for on-resin immobilisation with variable spacer length. E: Highly water-soluble sulfonated phenols, E1: potassium guaiacolsulfonate and E2: Tiron.

Initial studies towards the anti-fouling behaviour of these modified membranes focused on the adsorption of bovine serum albumin (BSA) as a protein model foulant. ${ }^{[129]}$ Grafted 4-HBA was particularly effective in minimising BSA adsorption, even to levels that were below the detection limit. Importantly, the modification with 4-HBA acid did not alter the water flux substantially. Studies on spin-coated PES further revealed a sharp decrease in fouling towards BSA, dextrin (polysaccharide) and tannin (polyphenol), especially at short modification times $(<2 \mathrm{~h}){ }^{[130]}$ This effect was more pronounced for 4-HBA than for gallic acid. Finally, repellence of two Listeria monocytogenes strains was tested by using the commonly employed EGD strain and the LR-991 strain, which is known for its tendency to form biofilms. ${ }^{[131]}$ I nitial attachment of both strains on surfaces modified with 4-HBA, FA and gallic acid was all reduced by approximately $60 \%$ compared to attachment on bare PES. Further biofilm formation was equally well reduced in the cases of FA and gallic acid modification. Attachment of both strains to modified surfaces under dynamic flow conditions resulted in an even larger reduction in all cases (70-95\%).

However, recent results from our group have shined new light on the plausibility of grafting. Laccase-mediated oligomerisation of 4-HBA was performed in the presence of insoluble (Scheme 24B) and soluble (Scheme 24C) PES model compounds to determine the nature of the covalent bond between PES and the phenolic compound. However, no covalent conjugate could be observed in either case. Additionally, the use of a more reactive phenol did not result in the observation of phenol/model PES adducts either. In order to mimic the architecture of PES more accurately, a PES model compound was immobilised on a resin, which was subsequently used as a PES mimic in laccase-mediated oligomerisation, and successively cleaved off from the resin to be analysed by LC-MS (Scheme 24D). However, this approach also did not result in any observation of a phenol/model PES conjugate. Finally, to impart additional functionality to PES membranes, laccase was reacted with sulfonated phenolics in the presence of the membrane (Scheme 24E). Although extensive colouration of the medium and substantial conversion of the starting material were observed, the membrane did not get coloured. The current hypothesis is that coating of PES is based on laccase-generated phenolic oligomers that adsorb strongly to the PES membrane due to their poor solubility and hydrophobicity. 
When completely soluble oligomers are generated (like those from sulfonated monomers), these stay in solution and do not adhere.

To summarise: due to the high diversity of synthetic oligomers used in laccase-mediated functionalisation, it is difficult to draw general conclusions. However, it is clear that modification of synthetic polymers and subsequent analysis can be interpreted in multiple ways. Any discrimination between grafting and adsorption should not be dealt with lightly, and, even if grafting seems likely, one has to conduct suitable control experiments to verify the results. Furthermore, many of the mentioned plastics could not be functionalised directly, but needed pre-activation. This severely increases the carbon footprint of the functionalisation approach.

\section{Conclusions}

It has become clear that laccase-mediated modification of surfaces can benefit many applications. Properties that are altered or conferred by doing so include colour, mechanical strength, anti-oxidant effects, anti-bacterial effects, etc. The power of laccase clearly lies in its ability to generate radicals in a mild way, and to produce water as the only by-product, while possessing a huge substrate scope and high solvent tolerance. If laccases start being produced on a larger scale, they will become cheaper, and might therefore, also be proposed as eco-friendly alternatives to inorganic or oil-based catalysts. Furthermore, as many of the materials susceptible to laccase-mediated modification are now being considered as waste, recycling through functionalisation by laccase is highly attractive.

Grafting, nonetheless, is not the main origin of alteration of the material in all mentioned applications. Grafting often coincides with adsorption, resulting in a material that is altered due to both types of modification. Strong, persistent binding without the influence of adsorbents is, however, frequently required in these applications. If the appropriate washing procedure is chosen, this might already be enough to remove any loosely bound molecules. Additionally, one has to consider the influence of reactions in solution: formed homo-oligomers might precipitate; as a result, control experiments in which only monomer is employed will not ensure a fair comparison. Grafting might also result in reversible covalent bonding, as is the case for imine formation. For many applications, irreversibility and thus, stability is crucial; therefore, one has to tune the grafting strategy to the desired application. Discrimination between grafting and adsorption is not easy, so the analytical tools employed to do so should be chosen wisely. FTIR analysis, for example, is much more valuable for examining grafting on polysaccharides than it is for examining grafting on lignocelluloses, since in the former, functional group transformation (as in the formation of imines) is employed more frequently. Ideally, all modified surfaces are examined with a range of surface-sensitive techniques, including XPS, FTIR, SWCA, Py-GC-MS or complementary techniques. Furthermore, the use of ambient MS to study covalently bound molecules on a surface is a promising technique that would certainly be of great use in the characterisation of materials generated through laccase-mediated grafting. ${ }^{[132]}$

Among all materials studied, lignocelluloses currently provide the best platform for grafting. However, this conclusion can only be drawn because this chemistry is well understood. Burton et al. set a benchmark approximately seven years ago by indicating that laccase-mediated grafting (and coupling) is indeed a promising technique to mildly modify several materials for a plethora of applications. ${ }^{[13 c]}$ This prediction has indeed been shown to be true. Given its rapidly increasing potential, it is therefore now time to think again beyond the applications and study its chemistry in much more detail, with many more techniques. This will provide new insights into how to make sure that functionalisation is really covalent, and can persistently yield the desired surface modification.

\section{Acknowledgements}

This project was funded by the NanoNextNL project 04A.01 "Biomimetic selective layers". 


\section{References}

[1] K. Horie, M. Baron, R. B. Fox, J. He, M. Hess, J. Kahovec, T. Kitayama, P. Kubisa, E. Marechal, W. Mormann, R. F. T. Stepto, D. Tabak, J. Vohlidal, E. S. Wilks, W. J. Work, G. Allegra, A. Fradet, K. Hatada, A. D. Jenkins, J. I. J in, R. G. Jones, P. Kratochvil, E. Marcechal, I. Meisel, W. V. Metanomski, G. Moad, S. Penczek, L. P. Rebelo, M. Rinaudo, I. Schopov, M. Schubert, V. P. Shibaev, S. Slomkowskj, K. Dorfner, M. J. Frechet, W. I. Harris, P. Hodge, T. Nishikubo, C. K. Ober, E. Reichmanis, D. C. Sherrington, M. Tomoi, D. Wohrle, Pure Appl. Chem. 2004, 76, 889-906.

[2] X. Liu, Q. Wu, Polymer 2001, 42, 10013-10019.

[3] D. Zang, F. Liu, M. Zhang, X. Niu, Z. Gao, C. Wang, Chem. Eng. J. 2015, 262, 210-216.

[4] A. Carlmark, E. E. Malmström, Biomacromolecules 2003, 4, 1740-1745.

[5] A. Hüttermann, C. Mai, A. Kharazipour, Appl. Microbiol. Biotechnol. 2001, 55, 387-394.

[6] B. Zhao, W. J. Brittain, Prog. Polym. Sci. 2000, 25, 677-710.

[7] H. Petersen, P. M. Fechner, A. L. Martin, K. Kunath, S. Stolnik, C. J. Roberts, D. Fischer, M. C. Davies, T. Kissel, Bioconjugate Chem. 2002, 13, 845-854.

[8] S. C. Lange, E. van Andel, M. M. J. Smulders, H. Zuilhof, Langmuir 2016, 32, 10199-10205.

[9] D. Neugebauer, Y. Zhang, T. Pakula, S. S. Sheiko, K. Matyjaszewski, Macromolecules 2003, 36, 6746-6755.

[10] K. Matyjaszewski, Macromolecules 2012, 45, 4015-4039.

[11] a) J. O. Zoppe, N. C. Ataman, P. Mocny, J. Wang, J. Moraes, H.-A. Klok, Chem. Rev. 2017, 117, 1105-1318; b) W.-L. Chen, R. Cordero, H. Tran, C. K. Ober, Macromolecules 2017, 50, 4089-4113; c) J. Niu, D. J. Lunn, A. Pusuluri, J. I. Yoo, M. A. O'Malley, S. Mitragotri, H. T. Soh, C. J. Hawker, Nat. Chem. 2017, 9, 537-545.

[12] P. S. Zhong, N. Widjojo, T.-S. Chung, M. Weber, C. Maletzko, J. Membr. Sci. 2012, 417-418, 52-60.

[13] a) G. S. Nyanhongo, T. Kudanga, E. Nugroho Prasetyo, G. M. Guebitz in Biofunctionalization of Polymers and Their Applications (Eds.: G. S. Nyanhongo, W. Steiner, G. Guebitz), Springer, Berlin, 2011, pp. 47-68; b) A. Mikolasch, F. Schauer, Appl. Microbiol. Biotechnol. 2009, 82, 605-624; c) T. Kudanga, G. S. Nyanhongo, G. M. Guebitz, S. Burton, Enzyme Microb. Technol. 2011, 48, 195-208; d) G. M. Guebitz, A. Cavaco-Paulo, Trends Biotechnol. 2008, 26, 32-38.

[14] V. Madhavi, S. S. Lele, Bioresources 2009, 4, 1694-1717.

[15] H. Yoshida, J. Chem. Soc. Trans. 1883, 43, 472-486.

[16] S. Kobayashi, H. Uyama, R. I keda, Chem.-Eur. J. 2001, 7, 4755-4760.

[17] M. R. Coy, T. Z. Salem, J. S. Denton, E. S. Kovaleva, Z. Liu, D. S. Barber, J. H. Campbell, D. C. Davis, G. W. Buchman, D. G. Boucias, M. E. Scharf, Insect Biochem. Mol. Biol. 2010, 40, 723732.

[18] N. Santhanam, J. M. Vivanco, S. R. Decker, K. F. Reardon, Trends Biotechnol. 2011, 29, 480489.

[19] a) C. M. Rivera-Hoyos, E. D. Morales-Álvarez, R. A. Poutou-Piñales, A. M. Pedroza-Rodríguez, R. RodrÍguez-Vázquez, J. M. Delgado-Boada, Fungal Biol. Rev. 2013, 27, 67-82; b) P. Baldrian, FEMS Microbiol. Rev. 2006, 30, 215-242; c) S. V. S. Kumar, P. S. Phale, S. Durani, P. P. Wangikar, Biotechnol. Bioeng. 2003, 83, 386-394.

[20] a) S. Witayakran, A. J. Ragauskas, Adv. Synth. Catal. 2009, 351, 1187-1209; b) J. R. J eon, P. Baldrian, K. Murugesan, Y. S. Chang, Microb. Biotechnol. 2012, 5, 318-332.

[21] M. Mogharabi, M. A. Faramarzi, Adv. Synth. Catal. 2014, 356, 897-927.

[22] S. Camarero, D. Ibarra, Á. T. Martínez, J. Romero, A. Gutiérrez, J. C. del Río, Enzyme Microb. Technol. 2007, 40, 1264-1271.

[23] a) R. Sarma, M. S. Islam, A.-F. Miller, D. Bhattacharyya, ACS Appl. Mater. Interfaces 2017, 9, 14858-14867; b) X. Zhang, M. Hua, L. Lv, B. Pan, Sci. Rep. 2015, 5, 8253; c) L. Lloret, G. 
Eibes, T. A. Lú-Chau, M. T. Moreira, G. Feijoo, J. M. Lema, Biochem. Eng. J. 2010, 51, 124131.

[24] J. Polak, A. Jarosz-Wilkolazka, Process Biochem. 2012, 47, 1295-1307.

[25] N. Nakajima, Y. I kada, Bioconjugate Chem. 1995, 6, 123-130.

[26] A. Dong, X. Fan, Q. Wang, Y. Yu, A. Cavaco-Paulo, Int. J. Biol. Macromol. 2015, 79, 353-362.

[27] S. V. Vassilev, D. Baxter, L. K. Andersen, C. G. Vassileva, T. J. Morgan, Fue/ 2012, 94, 1-33.

[28] H. F. Lecoanet, J.-Y. Bottero, M. R. Wiesner, Environ. Sci. Technol. 2004, 38, 5164-5169.

[29] L. Munk, A. K. Sitarz, D. C. Kalyani, J. D. Mikkelsen, A. S. Meyer, Biotechnol. Adv. 2015, 33, 13-24.

[30] I. Banerjee, R. C. Pangule, R. S. Kane, Adv. Mater. 2011, 23, 690-718.

[31] R. Kaegi, B. Sinnet, S. Zuleeg, H. Hagendorfer, E. Mueller, R. Vonbank, M. Boller, M. Burkhardt, Environ. Pollut. 2010, 158, 2900-2905.

[32] J. Pérez, J. Muñoz-Dorado, T. de la Rubia, J. Martínez, Int. Microbiol. 2002, 5, 53-63.

[33] E. M. Cadena, X. Du, G. Gellerstedt, J. Li, A. Fillat, J. García-Ubasart, T. Vidal, J. F. Colom, Bioresour. Technol. 2011, 102, 3911-3917.

[34] S. Camarero, O. García, T. Vidal, J. Colom, J. C. del Río, A. Gutiérrez, J. M. Gras, R. Monje, M. J. Martínez, Á. T. Martínez, Enzyme Microb. Technol. 2004, 35, 113-120.

[35] Ú. Fillat, D. I barra, M. Eugenio, A. Moreno, E. Tomás-Pejó, R. Martín-Sampedro, Fermentation 2017, 3, 17.

[36] T. Kudanga, E. Nugroho Prasetyo, J. Sipilä, P. Nousiainen, P. Widsten, A. Kandelbauer, G. S. Nyanhongo, G. M. Guebitz, Eng. Life Sci. 2008, 8, 297-302.

[37] T. Kudanga, E. Nugroho Prasetyo, J. Sipilä, G. S. Nyanhongo, G. M. Guebitz, Enzyme Microb. Technol. 2010, 46, 272-280.

[38] T. Kudanga, E. Nugroho Prasetyo, P. Widsten, A. Kandelbauer, S. J ury, C. Heathcote, J. Sipilä, H. Weber, G. S. Nyanhongo, G. M. Guebitz, Bioresour. Technol. 2010, 101, 2793-2799.

[39] T. Kudanga, E. Nugroho Prasetyo, J. Sipilä, A. Eberl, G. S. Nyanhongo, G. M. Guebitz, J. Mol. Catal. B 2009, 61, 143-149.

[40] K. Manda, D. Gördes, A. Mikolasch, E. Hammer, E. Schmidt, K. Thurow, F. Schauer, Appl. Microbiol. Biotechnol. 2007, 76, 407-416.

[41] T. Kudanga, E. Nugroho Prasetyo, J. Sipilä, G. M. Guebitz, G. S. Nyanhongo, J. Biotechnol. 2010, 149, 81-87.

[42] H. E. Schoemaker, P. J. Harvey, R. M. Bowen, J. M. Palmer, FEBS Lett. 1985, 183, 7-12.

[43] E. Aracri, A. Fillat, J. F. Colom, A. Gutiérrez, J. C. del Río, Á. T. Martínez, T. Vidal, Bioresour. Technol. 2010, 101, 8211-8216.

[44] W. R. Russell, A. R. Forrester, A. Chesson, M. J. Burkitt, Arch. Biochem. Biophys. 1996, 332, 357-366.

[45] J. Rencoret, E. Aracri, A. Gutiérrez, J. C. del Río, A. L. Torres, T. Vidal, A. T. Martínez, Biochem. Eng. J. 2014, 86, 16-23.

[46] M. Zhao, J. Li, E. Mano, Z. Song, D. M. Tschaen, E. J. J. Grabowski, P. J. Reider, J. Org. Chem. 1999, 64, 2564-2566.

[47] L. Munk, A. M. Punt, M. A. Kabel, A. S. Meyer, RSC Adv. 2017, 7, 3358-3368.

[48] E. A. Capanema, M. Y. Balakshin, J. F. Kadla, J. Agric. Food Chem. 2004, 52, 1850-1860.

[49] S. Kim, C. López, G. M. Güebitz, A. Cavaco-Paulo, Eng. Life Sci. 2008, 8, 324-330.

[50] J. Forde, E. Tully, A. Vakurov, T. D. Gibson, P. Millner, C. Ó'Fágáin, Enzyme Microb. Technol. 2010, 46, 430-437.

[51] G. Elegir, A. Kindl, P. Sadocco, M. Orlandi, Enzyme Microb. Technol. 2008, 43, 84-92.

[52] P. Widsten, C. Heathcote, A. Kandelbauer, G. M. Guebitz, G. S. Nyanhongo, E. Nugroho Prasetyo, T. Kudanga, Process Biochem. 2010, 45, 1072-1081.

[53] M. Schroeder, N. Aichernig, G. M. Guebitz, V. Kokol, Biotechnol. J. 2007, 2, 334-341. 
[54] C. Silva, T. Matamá, S. Kim, J. Padrão, E. Nugroho Prasetyo, T. Kudanga, G. S. Nyanhongo, G. M. Guebitz, M. Casal, A. Cavaco-Paulo, React. Funct. Polym. 2011, 71, 713-720.

[55] A. Fillat, O. Gallardo, T. Vidal, F. I. J. Pastor, P. Díaz, M. B. Roncero, Carbohydr. Polym. 2012, 87, 146-152.

[56] M. Schubert, J. Engel, L. Thöny-Meyer, F. W. M. R. Schwarze, J. Ihssen, Appl. Environ. Microbiol. 2012, 78, 7267-7275.

[57] A. Francesko, L. Blandón, M. Vázquez, P. Petkova, J. Morató, A. Pfeifer, T. Heinze, E. Mendoza, T. Tzanov, ACS Appl. Mater. Interfaces 2015, 7, 9792-9799.

[58] S. P. Silva, M. A. Sabino, E. M. Fernandes, V. M. Correlo, L. F. Boesel, R. L. Reis, Int. Mater. Rev. 2005, 50, 345-365.

[59] L. V. Garcia Peña, P. Petkova, R. Margalef-Marti, M. Vives, L. Aguilar, A. Gallegos, A. Francesko, I. Perelshtein, A. Gedanken, E. Mendoza, J. C. Casas-Zapata, J. Morató, T. Tzanov, Ind. Eng. Chem. Res. 2017, 56, 3599-3606.

[60] A. Dong, Y. Yu, J. Yuan, Q. Wang, X. Fan, Appl. Surf. Sci. 2014, 301, 418-427.

[61] X. Ni, A. Dong, X. Fan, Q. Wang, Y. Yu, A. Cavaco-Paulo, Fibers Polym. 2015, 16, 2276-2283.

[62] M. Fernández-Fernández, M. Á. Sanromán, D. Moldes, J. Wood Chem. Technol. 2015, 35, 156-165.

[63] J. Garcia-Ubasart, A. Esteban, C. Vila, M. B. Roncero, J. F. Colom, T. Vidal, Bioresour. Technol. 2011, 102, 2799-2803.

[64] J. Garcia-Ubasart, J. F. Colom, C. Vila, N. G. Hernández, M. Blanca Roncero, T. Vidal, Bioresour. Technol. 2012, 112, 341-344.

[65] C. Reynaud, S. Tapin-Lingua, G. Elegir, M. Petit-Conil, S. Baumberger, J. Biotechnol. 2013, 167, 302-308.

[66] S. Witayakran, A. J. Ragauskas, Enzyme Microb. Technol. 2009, 44, 176-181.

[67] A. Dong, J. Yuan, Q. Wang, X. Fan, J. Appl. Polym. Sci. 2014, 131, 40387.

[68] C. Mai, A. Majcherczyk, A. Hüttermann, Enzyme Microb. Technol. 2000, 27, 167-175.

[69] G. Gao, M. A. Karaaslan, J. F. Kadla, F. Ko, Green Chem. 2014, 16, 3890-3898.

[70] M. Schubert, P. Ruedin, C. Civardi, M. Richter, A. Hach, H. Christen, PLoS One 2015, 10, e0128623.

[71] M. Euring, M. Rühl, N. Ritter, U. Kües, A. Kharazipour, Biotechnol. J. 2011, 6, 1253-1261.

[72] S. Slagman, J. Escorihuela, H. Zuilhof, M. C. R. Franssen, RSC Adv. 2016, 6, 99367-99375.

[73] E. Aracri, C. Díaz Blanco, T. Tzanov, Green Chem. 2014, 16, 2597-2603.

[74] G. D. Love, C. E. Snape, M. C. Jarvis, I. M. Morrison, Phytochemistry 1994, 35, 489-491.

[75] L. Viikari, K. Kruus, J. Buchert (Valtion Teknillinen Tutkimuskeskus), WO 023117 A1, 1999

[76] S. A. Tromp, I. Matijošytė, R. A. Sheldon, I. W. C. E. Arends, G. Mul, M. T. Kreutzer, J. A. Moulijn, S. de Vries, ChemCatChem 2010, 2, 827-833.

[77] I. Patel, R. Ludwig, D. Haltrich, T. Rosenau, A. Potthast, Holzforschung 2011, 65, 475-481.

[78] S. Xu, Z. Song, X. Qian, J. Shen, Cellulose 2013, 20, 2371-2378.

[79] Y. Yu, Q. Wang, J. Yuan, X. Fan, P. Wang, Carbohydr. Polym. 2016, 153, 463-470.

[80] E. Aracri, T. Vidal, A. J. Ragauskas, Carbohydr. Polym. 2011, 84, 1384-1390.

[81] J. Jiang, W. Ye, L. Liu, Z. Wang, Y. Fan, T. Saito, A. Isogai, Biomacromolecules 2017, 18, 288-294.

[82] E. Aracri, T. Vidal, Cellulose 2012, 19, 867-877.

[83] E. Aracri, C. Valls, T. Vidal, Carbohydr. Polym. 2012, 88, 830-837.

[84] D. Jaušovec, R. Vogrinčič, V. Kokol, Carbohydr. Polym. 2015, 116, 74-85.

[85] Y. Yu, Q. Wang, J. Yuan, X. Fan, P. Wang, L. Cui, Carbohydr. Polym. 2016, 137, 549-555.

[86] C. Guimarães, S. Kim, C. Silva, A. Cavaco-Paulo, Biotechnol. J. 2011, 6, 1272-1279.

[87] C. Díaz Blanco, M. D. González, J. M. D. Monmany, T. Tzanov, Enzyme Microb. Technol. 2009, 44, 380-385.

[88] J. Liu, R. Pelton, J. M. Obermeyer, A. Esser, Biomacromolecules 2013, 14, 2953-2960. 
[89] S. Shi, R. Pelton, Q. Fu, S. Yang, Ind. Eng. Chem. Res. 2014, 53, 4748-4754.

[90] J. Garcia-Ubasart, T. Vidal, A. L. Torres, O. J. Rojas, Biomacromolecules 2013, 14, 1637-1644.

[91] Y. Zhang, A. Dong, Q. Wang, X. Fan, A. Cavaco-Paulo, Y. Zhang, Appl. Biochem. Biotechnol. 2014, 174, 820-831.

[92] Y. Zhang, X. Fan, Q. Wang, A. Cavaco-Paulo, RSC Adv. 2016, 6, 49272-49280.

[93] H. M. N. Iqbal, G. Kyazze, T. Tron, T. Keshavarz, Macromol. Mater. Eng. 2015, 300, 712-720.

[94] H. M. N. Iqbal, G. Kyazze, I. C. Locke, T. Tron, T. Keshavarz, Green Chem. 2015, 17, 38583869.

[95] H. M. N. Iqbal, G. Kyazze, T. Tron, T. Keshavarz, Cellulose 2014, 21, 3613-3621.

[96] H. M. N. Iqbal, G. Kyazze, I. C. Locke, T. Tron, T. Keshavarz, Int. J. Biol. Macromol. 2015, 81, 552-559.

[97] a) H. M. N. Iqbal, G. Kyazze, I. C. Locke, T. Tron, T. Keshavarz, Carbohydr. Polym. 2015, 131, 197-207; b) H. M. N. Iqbal, G. Kyazze, I. C. Locke, T. Tron, T. Keshavarz, eXPRESS Polym. Lett. 2015, 9, 764-772.

[98] H. M. N. Iqbal, G. Kyazze, T. Tron, T. Keshavarz, Polym. Chem. 2014, 5, 7004-7012.

[99] M. Rinaudo, Prog. Polym. Sci. 2006, 31, 603-632.

[100] R. Shepherd, S. Reader, A. Falshaw, Glycoconjugate J. 1997, 14, 535-542.

[101] M. Božič, S. Gorgieva, V. Kokol, Carbohydr. Polym. 2012, 87, 2388-2398.

[102] M. Božič, S. Gorgieva, V. Kokol, Carbohydr. Polym. 2012, 89, 854-864.

[103] A. Aljawish, I. Chevalot, B. Piffaut, C. Rondeau-Mouro, M. Girardin, J. Jasniewski, J. Scher, L. Muniglia, Carbohydr. Polym. 2012, 87, 537-544.

[104] M. Yuan, I. Tanabe, J.-M. Bernard-Schaaf, Q.-Y. Shi, V. Schlegel, R. Schurhammer, P. A. Dowben, B. Doudin, L. Routaboul, P. Braunstein, NewJ. Chem. 2016, 40, 5782-5796.

[105] A. Aljawish, L. Muniglia, A. Klouj, J. Jasniewski, J. Scher, S. Desobry, Food Hydrocolloids 2016, 60, 551-558.

[106] a) A. Aljawish, L. Muniglia, I. Chevalot, Biotechnol. Prog. 2016, 32, 491-500; b) A. Aljawish, L. Muniglia, J. J asniewski, A. Klouj, J. Scher, I. Chevalot, Process Biochem. 2014, 49, 863-871.

[107] C. Yang, Y. Zhou, Y. Zheng, C. Li, S. Sheng, J. Wang, F. Wu, Int. J. Biol. Macromol. 2016, 87, 577-585.

[108] R. S. Asquith, Chemistry of Natural Protein Fibers, Plenum Press, New York, 1977.

[109] Z. Moldovan, E. Jover, J. M. Bayona, Anal. Chim. Acta 2002, 465, 359-378.

[110] J.-R. J eon, E.-J. Kim, K. Murugesan, H.-K. Park, Y.-M. Kim, J.-H. Kwon, W.-G. Kim, J.-Y. Lee, Y.-S. Chang, Microb. Biotechnol. 2010, 3, 324-335.

[111] M. Yuan, Q. Wang, J. Shen, E. Smith, R. Bai, X. Fan, Text. Res. J. DOI: https://dx.doi.org/10.1177/0040517517712096.

[112] T. Zhang, R. Bai, J. Shen, Q. Wang, P. Wang, J. Yuan, X. Fan, Text. Res. J. DOI: https://dx.doi.org/10.1177/0040517517720497.

[113] T. Zhang, R. Bai, Q. Wang, X. Fan, P. Wang, J. Yuan, Y. Yu, Color. Technol. 2017, 133, 65-72.

[114] K. M. G. Hossain, M. D. González, A. R. Juan, T. Tzanov, Enzyme Microb. Technol. 2010, 46, 326-330.

[115] K. M. G. Hossain, M. D. González, G. R. Lozano, T. Tzanov, J. Biotechnol. 2009, 141, 58-63.

[116] K. M. G. Hossain, M. D. González, J. M. D. Monmany, T. Tzanov, J. Mol. Catal. B 2010, 67, 231-235.

[117] J. W. Ledbetter, J. Phys. Chem. 1977, 81, 54-59.

[118] H. Lee, S. M. Dellatore, W. M. Miller, P. B. Messersmith, Science 2007, 318, 426-430.

[119] W. Jia, Q. Wang, X. Fan, A. Dong, Y. Yu, P. Wang, RSC Adv. 2017, 7, 12977-12983.

[120] J. Shao, J. Liu, J. Zheng, C. M. Carr, Polym. Int. 2002, 51, 1479-1483.

[121] H. Shin, G. M. Guebitz, A. Cavaco-Paulo, Macromol. Mater. Eng. 2001, 286, 691-694.

[122] M. Schroeder, E. Fatarella, J. Kovač, G. M. Guebitz, V. Kokol, Biomacromolecules 2008, 9, 2735-2741. 
[123] C. Díaz Blanco, A. Ortner, R. Dimitrov, A. Navarro, E. Mendoza, T. Tzanov, ACS Appl. Mater. Interfaces 2014, 6, 11385-11393.

[124] I. Gonçalves, T. Matamá, A. Cavaco-Paulo, C. Silva, Biocatal. Biotransform. 2014, 32, 2-12.

[125] E. H. Acero, D. Ribitsch, R. D. Rodriguez, A. Dellacher, S. Zitzenbacher, A. Marold, K. J. Greimel, M. Schroeder, A. Kandelbauer, S. Heumann, G. S. Nyanhongo, H. Schwab, G. M. Guebitz, J. Mol. Catal. B 2012, 79, 54-60.

[126] H. Flemming, Appl. Microbiol. Biotechnol. 2002, 59, 629-640.

[127] I.-S. Chang, P. L. Clech, B. J efferson, S. Judd, J. Environ. Eng. 2002, 128, 1018-1029.

[128] N. Nady, K. Schroën, M. C. R. Franssen, B. van Lagen, S. Murali, R. M. Boom, M. S. Mohyeldin, H. Zuilhof, ACS Appl. Mater. Interfaces 2011, 3, 801-810.

[129] N. Nady, K. Schroën, M. C. R. Franssen, M. S. Mohyeldin, H. Zuilhof, R. M. Boom, J. Membr. Sci. 2012, 394, 69-79.

[130] N. Nady, K. Schroën, M. C. R. Franssen, R. Fokkink, M. S. Mohyeldin, H. Zuilhof, R. M. Boom, J. Colloid Interface Sci. 2012, 378, 191-200.

[131] S. van der Veen, N. Nady, M. C. R. Franssen, H. Zuilhof, R. M. Boom, T. Abee, K. Schroën, J. Appl. Polym. Sci. 2015, 132, 41576.

[132] a) R. K. Manova, S. Joshi, A. Debrassi, N. S. Bhairamadgi, E. Roeven, J. Gagnon, M. N. Tahir, F. W. Claassen, L. M. W. Scheres, T. Wennekes, K. Schroën, T. A. van Beek, H. Zuilhof, M. W. F. Nielen, Anal. Chem. 2014, 86, 2403-2411; b) R. Sen, J. Escorihuela, M. M. J. Smulders, H. Zuilhof, Langmuir 2016, 32, 3412-3419. 


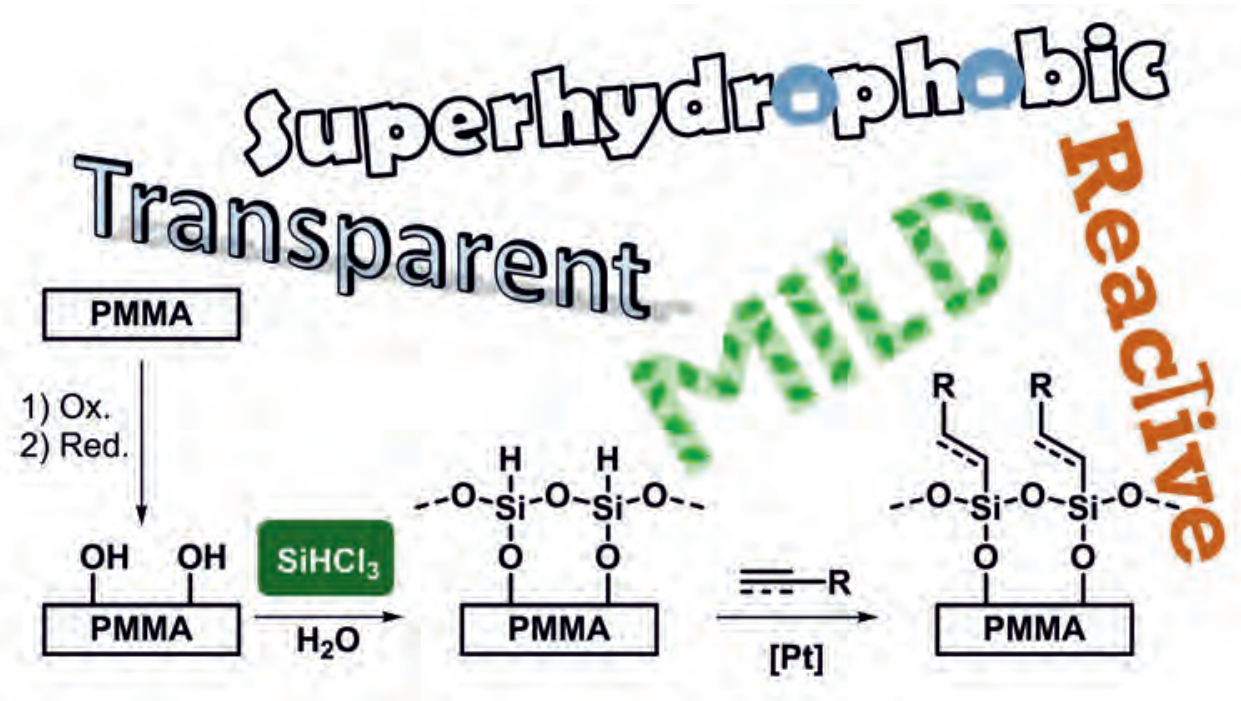




\title{
Chapter 5
}

\section{One-step generation of reactive superhydrophobic surfaces via $\mathrm{SiHCl}_{3}-$ based silicone nanofilaments}

\begin{abstract}
Superhydrophobic surfaces gain ever-growing attention due to their applicability in many (consumer) products/materials as they often display, among others, anti-fouling, anti-icing and/or self-cleaning properties. A simple way to achieve superhydrophobicity is through the growth of silicone nanofilaments. These nanofilaments, however, are very often non-reactive and thus difficult to utilise in subsequent chemistries. In response, we have developed a single-step procedure to grow intrinsically superhydrophobic ( $\mathrm{SiHCl}_{3}$-based) silicone nanofilaments with selective reactivity. The silicone nanofilaments could be further functionalised via Pt-catalysed hydrosilylation of exposed $\mathrm{Si}-\mathrm{H}$ moieties. These surfaces are easily obtained using mild conditions, and are stable under hydrolytic conditions (neutral water, $24 \mathrm{~h}$ at $80^{\circ} \mathrm{C}$ ), while remaining highly transparent, which makes them well suited for optical and photochemical experiments.
\end{abstract}

A minorly adjusted version of this chapter is accepted for publication as One-Step Generation of Reactive Superhydrophobic Surfaces via SiHCl3-Based Silicone Nanofilaments S. Slagman, S.P. Pujari, M.C.R. Franssen and H. Zuilhof 2018, accepted for publication in Langmuir

* This chapter is directly followed by its corresponding supporting information. 


\section{I ntroduction}

Since the beginning of the twenty-first century the production and use of superhydrophobic surfaces has gained tremendous attention. ${ }^{[1]}$ Superhydrophobic materials and coatings are of special interest as they can be anti-fouling ${ }^{[2]}$ and anti-icing/deicing, ${ }^{[3]}$ and can possess self-cleaning properties. ${ }^{[4]}$ Surface hydrophobicity is generally assessed by measuring the static water contact angle (SWCA). By definition, a superhydrophobic surface has an SWCA of $\geq 150^{\circ} .{ }^{[5]}$ Although applying low surface energy (perfluoroalkyl-based) coatings results in hydrophobic surfaces, these coatings generally do not have SWCA's of more than ca. $123^{\circ} .{ }^{[6]}$ In order to further increase the contact angle, and thus generate superhydrophobicity, the surface should be roughened through nano- or microtexturing. ${ }^{[7]}$ Furthermore, in many applications, such as microfluidics and optics, the optical transmittance of materials is of utmost importance. When superhydrophobic transparent materials are required, the process of making this material superhydrophobic should, thus, not result in a major loss of transparency.

In recent years, many techniques have been developed to induce roughness. By means of e.g. laser ablation ${ }^{[8]}$ or lithography ${ }^{[9]}$ highly defined micro-nano structures can be obtained. Supplementing this are bottom-up approaches in which a rough coating is, for example, obtained through spraying, ${ }^{[10]}$ spin-coating ${ }^{[11]}$, chemical vapor deposition ${ }^{[12]}$ or nanostructuring of preformed microstructures. ${ }^{[13]}$ Such micro-nanostructured surfaces are easily obtained through the growth of silicone nanofilaments (SNF's) by means of chemical vapor deposition or solution-phase deposition of trichlorosilanes or triethoxysilanes. ${ }^{[1,}{ }^{14]}$ SNF's are nanometer-thick randomly oriented twisted polysiloxane rods with a high surface area that can be grown from a multitude of materials. ${ }^{[1]}$ Due to their high surface area, materials coated with SNF's are rendered superhydrophobic while - in those cases where glass or a transparent polymer is used as support - maintaining high transparency. SNF length, surface coverage and surface density are easily regulated by varying water concentration, silane concentration, water/silane ratio or reaction time, which thus allows for a highly tunable one-step preparation of superhydrophobic surfaces. ${ }^{[1]]}$

Although multiple materials have been shown to support SNF formation, ${ }^{[14 b]}$ glass is by far the most common material to grow SNF's from ${ }^{[1]}$ due to its optical properties and widespread use. Interestingly, to the best of our knowledge, substantial SNF growth from pure PMMA has not been reported yet, likely related to its low compatibility with a range of organic solvents. Kasapgil et al.[14c] studied the growth of superhydrophobic SNF's on polyacrylates using $\mathrm{MeSiCl}_{3}$ in the gas phase and in solution, where, for PMMA, they obtained mostly spherical structures plus some nanofilaments. Easy access to superhydrophobic, functionalisable, transparent PMMA would be highly interesting given its widespread use in both outdoor applications as well as increasingly in microfluidics.

Surface activation to enhance SNF attachment is commonly achieved through air- or oxygenplasma treatment. ${ }^{[15]}$ By doing so, the surface is enriched with a plurality of oxygen-bearing functional groups, which act as anchor points for further functionalisation. ${ }^{[16]}$ Plasma treatment is, however, not suitable for the modification of microfluidic channels due to diffusion limitations. ${ }^{[17]}$ Furthermore, UVinitiated surface activation is not desirable for synthetic polymer surfaces due to yellowing and possible chain-scission. ${ }^{[18]}$ Within our group, we have recently developed a mild, liquid-based oxidation/reduction strategy that allows for the surface modification of microfluidic channels, and only introduces hydroxyl functionalities. ${ }^{[16]}$ Oxidation of a cyclic olefin copolymer (COC) surface under Fenton-like conditions using copper (II) acetate and hydrogen peroxide resulted in a multitude of surface-bound oxygen moieties that were reduced to hydroxyl groups upon subsequent employment of sodium borohydride in methanol.

SNF's are most commonly grown using trichloromethylsilane, yielding a surface with minimal follow-up reactivity. ${ }^{[14 b, 14 c]}$ While advantageous for some applications, this limits the scope of potential use significantly, since it is difficult to subsequently add tailor-made functional moieties or to apply mild, yet covalent, patterning. Harsh plasma treatments are typically needed to activate such a surface 
in order for it to be modified any further, e.g. through perfluoroalkane self-assembled monolayer (SAM) formation. ${ }^{[19]}$ The use of trichlorovinylsilane for the growth of SNF's does allow for further modification through photoinduced disulfide-ene or thiol-ene click chemistry, as demonstrated by Levkin and co-workers (so-called reactive SNF's). ${ }^{[20]}$ However, unlike trichloromethylsilane-based SNF's, trichlorovinylsilane-based SNF's grown from polymer surfaces have not been reported to be intrinsically superhydrophobic $\left(\right.$ SWCA $\left.<135^{\circ}\right)$. ${ }^{[20 a]}$ Co-polymerisation with fluoroalkane-substituted silanes $^{[21]}$ or further functionalisation is then required to generate superhydrophobicity. ${ }^{[20 a]}$ To overcome this, we hereby report the one-step generation of a transparent superhydrophobic reactive poly(methylmethacrylate) (PMMA) surface through the formation of trichlorosilane-based $\left(\mathrm{SiHCl}_{3}\right.$, also: trichlorohydrosilane) SNF's (Scheme 1). Additionally, we are able to further functionalise the resulting surface through Pt-catalysed hydrosilylation using the Si-H moieties in the SNF backbone. In this way we produce superhydrophobic reactive SNF's grown from a polymer surface in a single step.

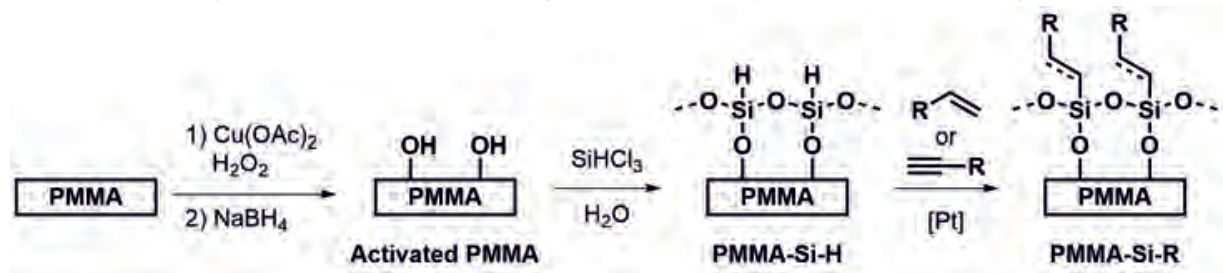

Scheme 1. Formation of functional superhydrophobic PMMA surfaces: mild activation, silicone nanofilament formation and subsequent functionalisation through $\mathrm{Pt}(0)$-catalysed hydrosilylation.

\section{Results and discussion}

\section{Mild activation of PMMA}

Oxidation of PMMA using mild peroxidative copper catalysis as developed for microfluidic devices $^{[16]}$ led to homogeneous yellowing of the PMMA surface, partially due to the presence of copper oxides. However, after sodium borohydride treatment, PMMA regained its original clarity. This mild oxidation/reduction strategy resulted in a near constant SWCA: from $72^{\circ}$ for PMMA to $68^{\circ}$ for activated PMMA. In addition, wide scan XPS analysis revealed only a minor overall increase in oxygen content (average of $1.0 \%$ increase, Figure $1 \mathrm{~A}$ and $1 \mathrm{~B}$ ). The significant change that nevertheless had taken place at the surface was only borne out at XPS $O$ 1s narrow scans. First of all, the $O$ is peak at $532.0 \mathrm{eV}$ was assigned to PMMA's carbonyl oxygen, while the peak at $533.5 \mathrm{eV}$ was assigned to its COC oxygen atom. This assignment was based on both literature ${ }^{[22]}$ and B3LYP/6-311+G(d,p) calculations, analogous to previous DFT-based methods to assign XPS peaks, which yield a calculated difference of $1.59 \mathrm{eV}$ between the binding energies of these two types of $\mathrm{O}$ atoms, in excellent agreement with experiment. ${ }^{[23]}$
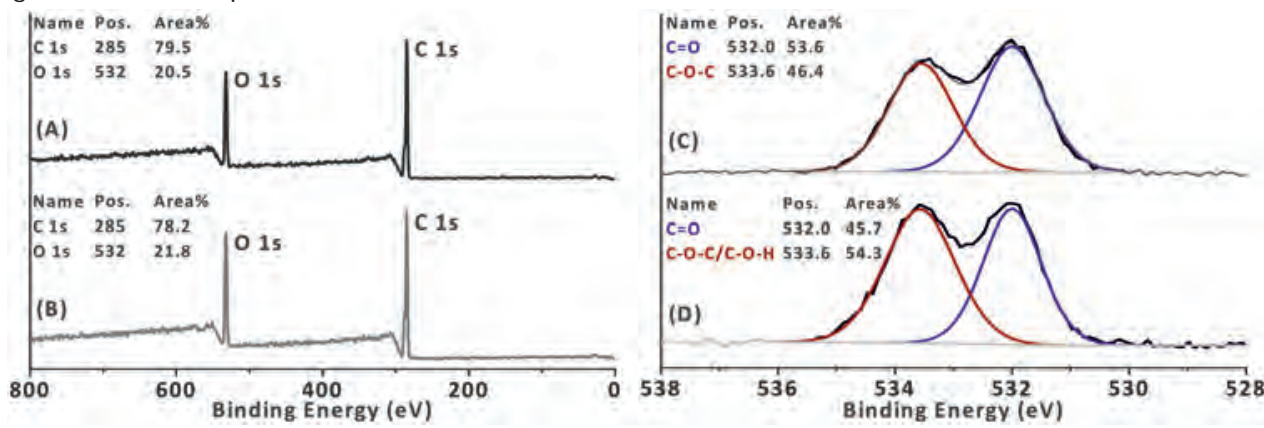

Figure 1. XPS wide scans of A: bare PMMA and B: activated PMMA. XPS O 1s narrow scans of C: bare PMMA and D: activated PMMA. 
As can be seen in Figure $1 \mathrm{C}$ and $1 \mathrm{D}$ the peak at $533.5 \mathrm{eV}$ increases (on average by $8 \%$ ) significantly after the oxidation/reduction sequence. For unmodified PMMA this peak only corresponds to the $\mathrm{C}-\mathrm{O}-\mathrm{C}$ oxygen atom of its ester moiety, where, after oxidation, this peak increases due to the introduction of alcohol functional groups $(\mathrm{C}-\mathrm{O}-\mathrm{H})$, which are expected at nearly the same binding energy (within $0.2 \mathrm{eV}$ ). Although the surface was significantly altered chemically, this activation procedure barely changed the surface topography: $<1.0 \mathrm{~nm}$ change in roughness; compare SI Figure $\mathrm{S} 2$ and S3.

\section{Silanisation of activated PMMA using $\mathrm{SiHCl}_{3}$}

Due to the presence of a reactive handle, we could now further functionalise the PMMA surface through silanisation. Again building on the work regarding $\mathrm{COC}$ modification, ${ }^{[16]}$ we initially envisioned the formation of a thin polysiloxane layer prepared from $\mathrm{SiHCl}_{3}$, to allow for further functionalisation through hydrosilylation. To our surprise, however, silanisation of activated PMMA using $\mathrm{SiHCl}_{3}$ resulted in a hydrophobic surface with a SWCA of approximately $130^{\circ}$. The silanisation conditions employed that led to this dramatic increase in SWCA were directly copied from the work regarding COC modification in which silane concentrations were high and the water concentration was not regulated. It is, however, well known that the degree of water is of utmost importance for satisfactory surface silanisation, as high concentrations will lead to the formation of (insoluble) homopolymers, whereas too low concentrations limit surface-grafted polysiloxane formation. ${ }^{[24]}$ Based on the conditions employed for the formation of trichloromethylsilane-based SNF's, water and $\mathrm{SiHCl}_{3}$ were hereafter used in equal concentrations. ${ }^{[14 b]}$ As can be seen in Figure $2 \mathrm{~A}$, controlling the trichlorosilane concentration $(3.5 \mathrm{mM})$ and performing the reaction in water-saturated cyclohexane $(3.5 \mathrm{mM})^{[25]}$ resulted in the reproducible generation of superhydrophobic PMMA-polysiloxane hybrids (PMMA-Si-H, SWCA $\left(9 \mu\right.$ ldroplets) $\left.=153-159^{\circ}\right) .3 \mu$ water droplets did not adhere to the PMMA-Si-H surface and in some cases this was also observed for $9 \mu$ ldroplets.
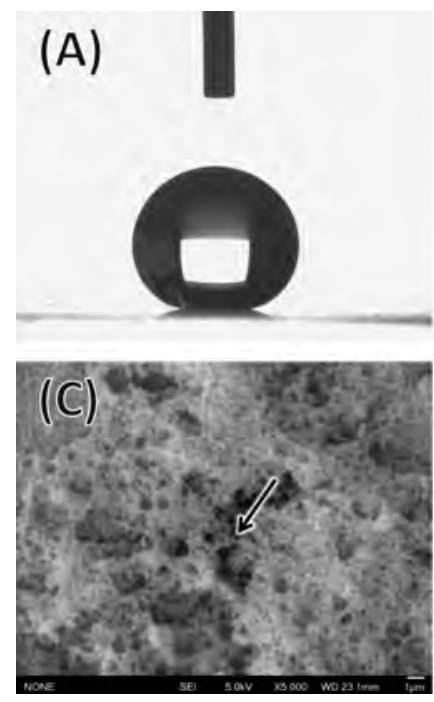
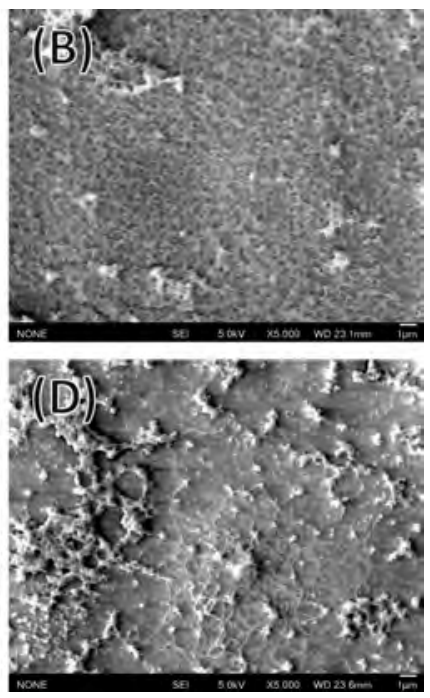

Figure 2. PMMA-Si-H resulting from silanisation using $3.5 \mathrm{mM} \mathrm{SiHCl}_{3}$ in water-saturated cyclohexane (3.5 $\left.\mathrm{mM} \mathrm{H}_{2} \mathrm{O}\right)$. A: Representative image of a $9 \mu \mathrm{l}$ water droplet on PMMA-Si$\mathrm{H}\left(\right.$ SWCA $\left.=158^{\circ}\right)$. B: SEM image depicting a homogeneous porous layer of condensed fibres. C: SEM image depicting a strand of polysiloxane fibres (indicated by an arrow) spanning a, locally porous, patch of denser fibres. D: SEM image of a newly initiated fibre network surrounded by more mature (denser) networks (SWCA = $\left.151^{\circ}\right)$.

These superhydrophobic surfaces were, furthermore, characterised by ultra-low water sliding angles; all modified surfaces exhibited sliding angles of $<1^{\circ}$. Even small defects, induced by scratching for example, did not exert large effects on the sliding angle (sliding angles remained < $15^{\circ}$ ). XPS-analysis revealed that, after silanising for $1 \mathrm{~h}$, the supporting PMMA layer could not be detected anymore (Figure 3A), indicating a dense polysiloxane network of at least $10 \mathrm{~nm}$ thickness (approximate maximum attenuation length of emitted electrons). Concomitantly, a strong IR signal at 
$\bar{v}=2251 \mathrm{~cm}^{-1}$ corresponding to an $\mathrm{O}_{3} \mathrm{Si}-\mathrm{H}$ stretching vibration was observed as clear indication of successful silanisation (Figure 3B and $3 \mathrm{C}$ ).

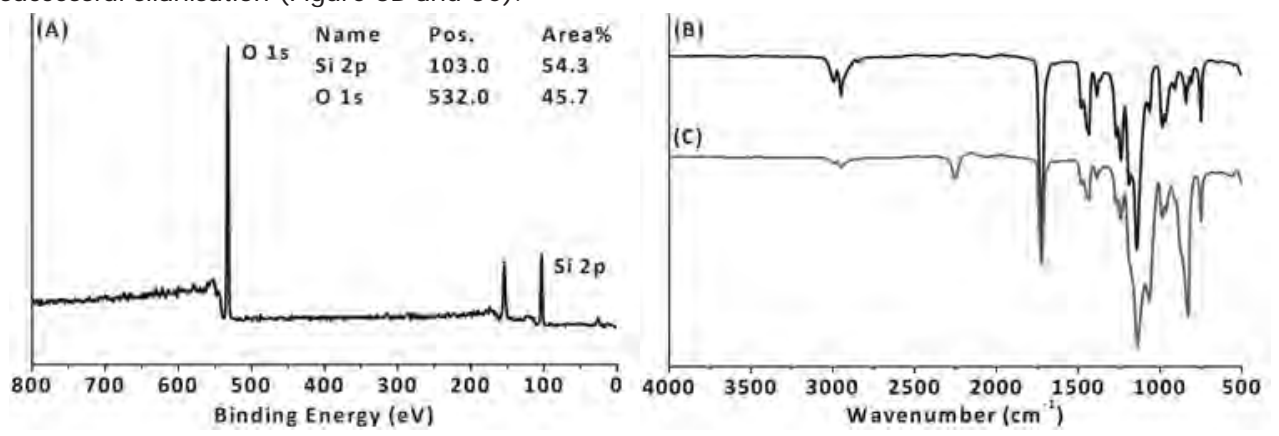

Figure 3. A: XPS wide scans of PMMA-Si-H and IR-spectra of B: activated PMMA and C: PMMA-Si-H.

Despite the presence of a thick polysiloxane coating, PMMA-Si-H proved to be highly transparent. The loss in optical transmittance of PMMA-Si-H compared to PMMA is, on average, limited to $<2 \%$ over the entire visible light range $(400-800 \mathrm{~nm})$, with a maximum loss of $4 \%$ at $400 \mathrm{~nm}$ (Figure 4 ). The modified surfaces are thus still highly suitable for photochemical or optical experiments, which is of particular interest to the microfluidics industry.

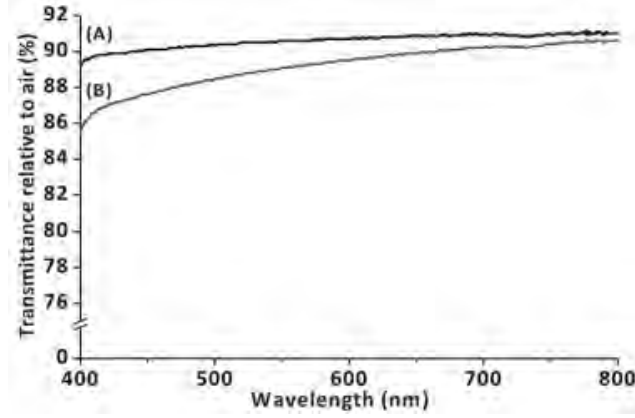

Figure 4. Visible-light transmittance relative to air of A: bare PMMA and B: PMMA-Si-H.

In order for the surface to become superhydrophobic, silanisation must have induced some form of roughness. ${ }^{[7]}$ The origin of this roughness might be mere deposition of $\mathrm{SiHCl}_{3}$-based polysiloxane nanoparticles, for example. To determine the roughening mechanism, we initially performed atomic force microscopy (AFM) studies on the superhydrophobic PMMA-polysiloxane hybrid. However, the dragging motion of the AFM tip induced artificial wells and peaks, most likely due to large differences in height between actual wells and peaks of the PMMA-Si-H surface (data not shown). ${ }^{[26]}$ Scanning electron microscopy (SEM) imaging provided a better picture of surface architecture (Figure 2B and 2C). The presence of a dense polysiloxane layer, as was hypothesised after XPS-analysis, was confirmed through SEM imaging, as a homogeneous porous layer of condensed fibres was visible (Figure 2B). This fibrous structure became even more clearly visible in areas with larger pores. Figure $2 \mathrm{C}$ displays a strand of polysiloxane fibres spanning a, locally porous, patch of denser fibres. By silanising the activated PMMA surface for a shorter period of time, initiation of the fibrous network from the flat surface could be visualised. Figure 2D depicts one of these initiation sites in which the network is growing outwards. We therefore hypothesise that silanisation is initiated locally from activated PMMA, and initial fibre growth occurs in a 1D-fashion. In addition, such fibres sporadically spawn cross-linking fibres in the second dimension, after which gradual growth and thickening of multiple networks results in complete surface coverage. Growth of polysiloxane fibres has been observed before; Seeger and co-workers have named these fibres silicone nanofilaments (SNF's). ${ }^{[14 b]}$ 
These SNF's are commonly prepared from small mono-alkyl-functionalised silanes bearing three hydrolysable groups, such as trichloromethylsilane. ${ }^{[14 b, 14 c]}$ However, the use of trichlorosilane $\left(\mathrm{SiHCl}_{3}\right)$ for the growth of SNF's has, to the best of our knowledge, not been reported before, while this presents a unique example of single-step superhydrophobisation through silicone nanofilament formation with a silane that does not bear any hydrophobic (hydrocarbon or fluorocarbon) moieties.

Although full surface coverage was not achieved for the surface depicted in Figure 2D, the surface did prove to be superhydrophobic $\left(\right.$ SWCA $\left.=151^{\circ}\right)$. This is, however, not always reproducible as some surfaces that had undergone the same treatment did not become superhydrophobic. A thicker homogeneous layer is thus preferred. Formation of such a layer furthermore eases analysis as carbon from PMMA could no longer be observed after silicone nanofilament growth (SI Figure S6B).

Silanisation of non-activated PMMA revealed the importance of the employed oxidation/reduction sequence. By comparing the $\mathrm{O}_{3} \mathrm{Si}-\mathrm{H}$ stretching vibration $\left(\bar{v}=2251 \mathrm{~cm}^{-1}\right)$ after silanisation to PMMA's $\mathrm{C}=\mathrm{O}$ stretching vibration $\left(\bar{v}=1724 \mathrm{~cm}^{-1}\right.$, signal intensity consistent before and after silanisation), a reasonable estimate of the degree of silanisation could be made. This ratio proved to be three times as large for PMMA-Si-H that had been $\mathrm{Cu}(\mathrm{II}) / \mathrm{H}_{2} \mathrm{O}_{2}$-activated prior to silanisation than for non-activated PMMA. The significance of prior surface activation to enhance silicone nanofilament growth has been addressed before by Seeger and co-workers. ${ }^{[27]}$ This observation thus confirms the advantage provided by non-destructive strategies as we employed here to activate delicate polymers like PMMA.

\section{Pt-catalysed functionalisation of PMMA-Si-H}

The presence of reactive $\mathrm{Si}-\mathrm{H}$ moieties on the PMMA-Si-H surface allows for further functionalisation of the SNF's through hydrosilylation. Reactive SNF's grown from a polymer surface that have been reported up to date are based on trichlorovinylsilane, which proved not be intrinsically superhydrophobic.[20a] Superhydrophobicity could, in those cases, only be achieved through subsequent high-energy light-triggered $(260 \mathrm{~nm})$ thiol-ene chemistry. Previous work regarding hydrosilylation of hydrocarbon polymer-polysiloxane hybrids used light-triggered $\mathrm{Si}-\mathrm{H}$ bond breaking. ${ }^{[16]}$ Such a UV light based approach would fail in the case of PMMA, as this absorbs UV light itself, leading to yellowing and/or chain-scission of the PMMA support layer. ${ }^{[18]}$ We therefore aimed to expand the scope of Si-C bond formation by employing (thermally activated) Pt-catalysis rather than photochemistry. For this we hypothesised that the use of low concentrations of Karstedt's catalyst (proposed structure: $\mathrm{Pt}(0)_{2}\left[\left(\mathrm{Me}_{2} \mathrm{SiCH}=\mathrm{CH}_{2}\right)_{2} \mathrm{O}\right]_{3}$ ), might allow for hydrosilylation without the presence of phenylsilanes in the polysiloxane backbone. ${ }^{[16]}$

As a proof-of-principle, we have used four alkenes and alkynes (A-D, Scheme 2) with different reactivity and degrees of fluorination to perform hydrosilylation reactions with, as these would allow for easy detection through $\mathrm{C}$ 1s and F 1s XPS analysis due to a high degree of fluorination.

(A)

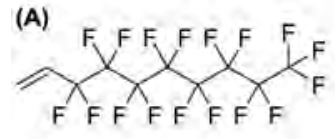

(B)

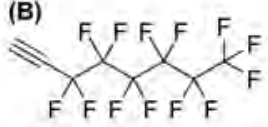

(C)

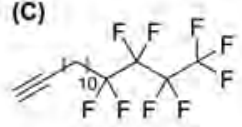

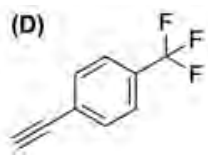

Scheme 2. Fluorinated alkenes and alkynes (A-D) used for hydrosilylation.

As expected, all surfaces functionalised with A, B and C (PMMA-Si-A, PMMA-Si-B and PMMA-Si-C) remained superhydrophobic after hydrosilylation (SWCA $(9 \mu \mathrm{L}) \mathbf{A}: 155^{\circ}, \mathbf{B}: 157^{\circ}$ and C: $157^{\circ}$ ). As for PMMA-Si- $\mathrm{H}$, for all these modified surfaces, sliding angles proved to be extremely low $\left(<1^{\circ}\right)$. Alkyne $\mathbf{D}$ is of special interest as it is the most reactive, but also a very challenging reagent since PMMA is known to dissolve in (structurally highly similar) toluene. ${ }^{[28]}$ Its use indeed led to surface functionalisation, but also to yellowing and cracking of the surface (results not shown). Research was therefore continued with compounds $\mathbf{A}$ to $\mathbf{C}$.

XPS analysis of PMMA-Si-A to PMMA-Si-C proved the success of hydrosilylation via the appearance of a distinct $F$ 1s peak at $688 \mathrm{eV}$ (Figure 5). As can be seen in Figure 5D, hydrosilylation of $\mathbf{A}$ results in 
far less fluorine $(\mathrm{F} / \mathrm{Si}=0.7)$ on the surface than hydrosilylation of $\mathbf{B}(\mathrm{F} / \mathrm{Si}=1.9)$ and $\mathbf{C}(\mathrm{F} / \mathrm{Si}=1.4)$ does. Furthermore, the introduction of a unique element (fluorine) allowed for a relative comparison of the quantitative success of hydrosilylation between A, B and C. By calculating the F/Si ratio and dividing that by the amount of fluorine atoms in the respective alkyne/alkene we can qualitatively derive the relative reactivity of $\mathbf{A}$ to $\mathbf{C}$ (Figure 5E). As expected, $\mathbf{A}$ was the least reactive, while alkynes $\mathbf{B}$ and $\mathbf{C}$ proved to be equally reactive, even though $\mathbf{B}$ bears strongly electron-withdrawing fluorine atoms in close proximity to the reactive alkyne.
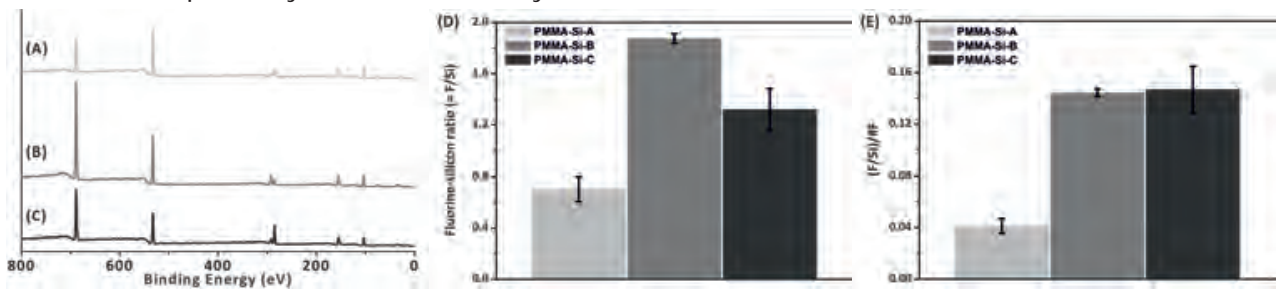

Figure 5. Result of Pt-catalysed hydrosilylation ( $24 \mathrm{~h}$ ); XPS wide scans of A: PMMA-Si-A, B: PMMA-Si-B and C: PMMASi-C (for area\% see SI Figures S7-S9). D: XPS-derived F/Si ratio and E: XPS-derived F/Si ratio divided by the number of fluorine atoms per respective molecule A-C.

Conversion of $\mathrm{Si}-\mathrm{H}$ moieties could be confirmed using IR spectroscopy as the signal at $\bar{v}=2251$ $\mathrm{cm}^{-1}$ was substantially smaller after hydrosilylation in all cases (Figure 6). As only the exterior of the thick and dense polysiloxane network is likely to be chemically accessible, Si-H moieties that are buried within this network were not converted, hence the observation of the respective IR signal. Even oxidation of some residual (exterior) $\mathrm{Si}-\mathrm{H}$ moieties during storage or use will not be of substantial influence on material properties, e.g. SWCA, as the formed Si-OH moieties will be shielded by a layer of fluoroalkanes/alkenes.

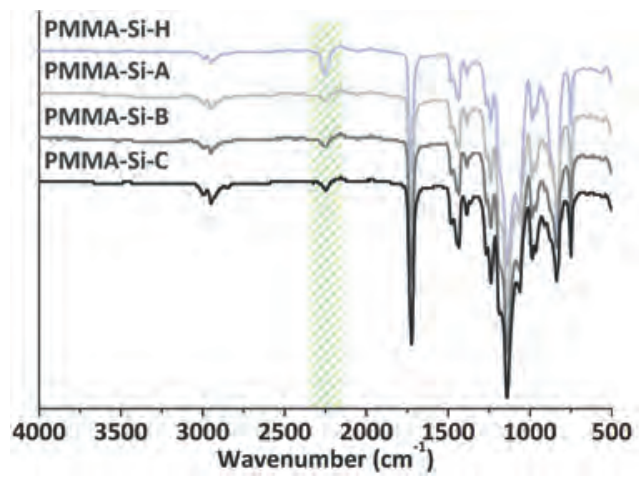

Figure 6. IR spectra of PMMA-Si-R compared to that of PMMA-Si-H with the signal at $\bar{v}=2251 \mathrm{~cm}^{-1}$, corresponding to $\mathrm{O}_{3} \mathrm{Si}-\mathrm{H}$.

Hydrosilylation results in only a small average reduction of the optical transmittance compared to PMMA-Si-H over the entire range of visible light (PMMA-Si-A $<4 \%$, PMMA-Si-B $<4 \%$, PMMA-Si-C $<$ $2 \%$ ), so also these surfaces are highly suitable for photochemical and optical experiments (Figure 7).

It could be shown that the nanofilamentous coating is stable under the derivatisation conditions as the XPS-derived O/Si ratios (1.1-1.3) obtained for the PMMA-polysiloxane hybrids are comparable to that of PMMA-Si-H. An increase of the O/Si ratio would have indicated that the PMMA support would become visible by XPS, so apparently the silicone nanofilaments do not break and leach out. Finally, SEM imaging confirms that hydrosilylation does not affect the polysiloxane topography (SI Figure S10). 


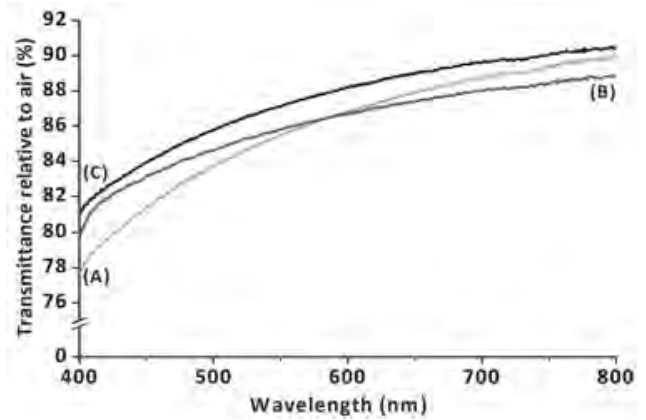

Figure 7. Visible light transmittance relative to air of A: PMMA-Si-A, B: PMMA-Si-B and C: PMMA-Si-C.

\section{Hydrolytic stability of PMMA-polysiloxane hybrids}

Apart from assessing whether the polysiloxane network was affected by hydrosilylation, we also evaluated the chemical stability of the material during use. The functionalised PMMA-polysiloxane hybrids should be able to withstand harsh hydrolytic conditions in order for them to be used effectively in microfluidic devices employing aqueous solutions. Their hydrolytic stability was assessed by heating PMMA-Si-A and PMMA-Si-C at $80^{\circ} \mathrm{C}$ at neutral $\mathrm{pH}$, under strongly acidic conditions (pH 3$)$ and under strongly basic conditions ( $\mathrm{pH} 11$ ), and measuring their SWCA afterwards (Figure 8). It could be shown that both surfaces were extremely stable in neutral water as their SWCA was not altered after $24 \mathrm{~h}$. Although their SWCA dropped considerably after being treated at $\mathrm{pH}$ 3, both PMMA-Si-A and PMMA-SiC remained hydrophobic (SWCA $=103^{\circ} \pm 8^{\circ}$ and $132^{\circ} \pm 6^{\circ}$, respectively). The effect of strongly basic conditions proved to be more profound as the SWCA of PMMA-Si-A dropped to $90^{\circ} \pm 5^{\circ}$ and that of PMMA-Si-C to $77^{\circ} \pm 6^{\circ}$.

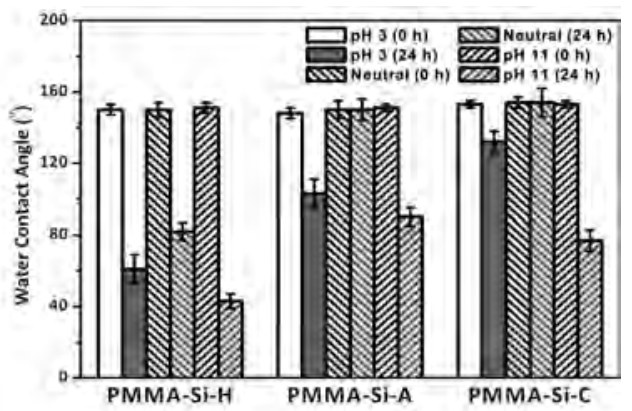

Figure 8. Static water contact angle (SWCA) of (fluorinated) PMMA-polysiloxane hybrids before and after being heated in water at three different degrees of acidity $\left(80^{\circ} \mathrm{C}, 24 \mathrm{~h}\right)$ as a measure for hydrolytic stability. Error bars indicate standard deviations of SWCA measured by fitting at least 10 times (Tangent-2) for at least two droplets on a single surface.

Hydrosilylation proved to be essential for preventing hydrolytic degradation of the polysiloxane layer as the SWCA of PMMA-Si-H dropped to $82^{\circ} \pm 5^{\circ}$ upon heating it in neutral water at $80^{\circ} \mathrm{C}$ for 24 $\mathrm{h}$ (Figure 8). At these high temperatures, the Si-H moieties of PMMA-Si-H are expected to be converted to polar Si-OH moieties, which thereby contributes to a lowering of the material's SWCA. A similar trend is observed for acid- and base-catalysed hydrolysis, as PMMA-Si-H becomes far more polar ( $61^{\circ} \pm 8^{\circ}$ and $43^{\circ} \pm 4^{\circ}$, respectively) than the fluorinated PMMA-polysiloxane hybrids do upon hydrolysis. A highly apolar, fluorine-rich, overlayer thus enhances stability, likely by hindering water from penetrating towards the polysiloxane backbone.

Hydrolytic stability could also be confirmed through XPS measurements as the spectra were practically identical before and after heating PMMA-Si-A and PMMA-Si-C at $80^{\circ} \mathrm{C}$ in neutral water (SI Figure 12). The stability is most characteristically borne out by appreciating that the O/Si ratio had 
barely been altered (Figure 9A; PMMA-Si-A/C; 1.2-1.4, PMMA-Si-A/C before; 1.1-1.3), which indicates minimal Si-O bond cleavage. Additionally, minimal changes in the percentage of surface-bound fluorine (Figure 9B; $<10 \%$ difference before and after heating) prove that the fluorine-rich overlayer stays intact. Although acidic hydrolysis of both PMMA-Si-A and PMMA-Si-C resulted in a considerable loss of hydrophobicity, chemically, their surfaces remained intact remarkably well (O/Si ratio $=1.4$ and $<$ $10 \%$ difference in the percentage of fluorine for both materials). Upon basic hydrolysis, the O/Si ratio increased significantly (3.1 and 6.7, respectively) and the percentage of fluorine was approximately halved, which is in line with the significant loss of hydrophobicity for both PMMA-Si-A and PMMA-Si-C.
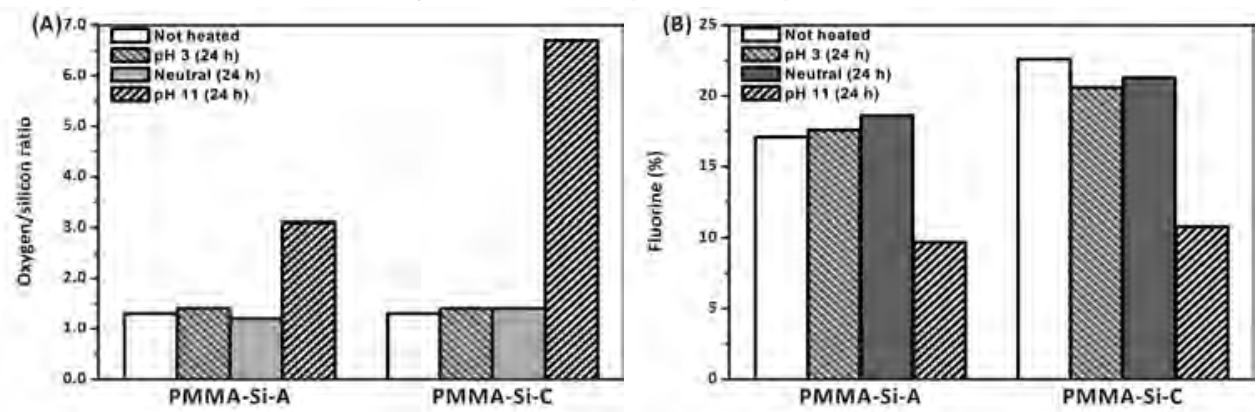

Figure 9. A: XPS-derived oxygen/silicon ratio and B: XPS-derived percentage of surface-bound fluorine of fluorinated PMMA-polysiloxane hybrids before and after being heated in water at three different degrees of acidity $\left(80^{\circ} \mathrm{C}, 24 \mathrm{~h}\right)$ as a measure for hydrolytic stability.

Finally, SEM imaging confirmed silicone nanofilament viability as the topography of both PMMA-SiA and PMMA-Si-C had not been altered upon either heating the material at $\mathrm{pH} 3$ (SI Figure 14), nor upon heating it in neutral water (SI Figure 15). As expected, basic hydrolysis resulted in a significant loss of the nanofilamentous coating (SI Figure 16). It concomitantly resulted in degradation of the PMMA support, which is apparent from the formation of micrometer-sized dents.

\section{Conclusions}

We report here on the formation of intrinsically superhydrophobic reactive silicone nanofilaments grown from a polymer surface. A mild oxidation/reduction strategy using $\mathrm{Cu}(\mathrm{II}) / \mathrm{H}_{2} \mathrm{O}_{2}$-based surface activation followed by reduction using sodium borohydride resulted in PMMA with a reactive handle to grow $\mathrm{SiHCl}_{3}$-based silicone nanofilaments from. Such silicone nanofilaments proved to be intrinsically superhydrophobic, even though the monomeric silane lacks any hydrophobic moiety. The resulting densely packed fibre network contains many reactive $\mathrm{Si}-\mathrm{H}$ moieties that could be functionalised through Pt-catalysed hydrosilylation with 1-alkenes or 1-alkynes. Such PMMA-polysiloxane hybrids remained highly optically transparent after both silanisation and hydrosilylation. Hydrosilylation, furthermore, proved to induce hydrolytic stability to the material as the fluorinated hybrids remained superhydrophobic upon hydrolysis ( $24 \mathrm{~h}$ ) in water at temperatures up to $80^{\circ} \mathrm{C}$. High transparency and hydrolytic stability make PMMA-polysiloxane hybrid surfaces suitable for further use, e.g. in a wide variety of microfluidic devices for optical and photochemical experiments.

\section{Experimental}

\section{Chemicals}

PMMA surfaces $(1 \times 1 \mathrm{~cm})$ were either generously provided by Micronit Microfluidics or obtained from Eriks and cut to size in-house. Cyclohexane (HPLC grade) was bought at LaboScientific B.V. Copper (II) acetate (98\%) was obtained from Alfa Aesar. Hydrogen peroxide (aqueous, 30\%) and sodium borohydride (flakes, $\geq 98 \%$ ) were purchased from Merck Millipore. Trichlorosilane $\left(\mathrm{SiHCl}_{3}\right)$ 
(99\%), Karstedt's catalyst (0.1 M in vinyl-terminated poly(dimethylsiloxane)) and ethylene glycol (anhydrous, 99.8\%) were obtained from Sigma Aldrich. WWR supplied $n$-hexane (99\%), while $1 H, 1 H, 2 H, 2 H$-perfluoro-1-decene A $(99 \%)$ and (perfluoro- $n$-hexyl)acetylene B $(95 \%)$ were bought at ABCR. 1-Ethynyl-4-(trifluoromethyl)benzene D (>98.0\%) was obtained from TCl. $13,13,14,14,15,15,16,16,16-N o n a f l u o r o-h e x a d e c-1$-yne C was synthesised according to a known procedure ${ }^{[29]}$ and purified prior to use by filtering it through a small plug of silica, Celite and magnesium sulfate (1:1:1), washing with cyclohexane and concentrating it in vacuo to obtain $\mathbf{C}$ as a colourless liquid.

\section{Surface modification}

PMMA surface activation. ${ }^{[16]}$ PMMA samples $(1 \times 1 \mathrm{~cm})$ were rinsed with cyclohexane before being sonicated $(37 \mathrm{kHz})$ in cyclohexane at room temperature for $5 \mathrm{~min}$. After being rinsed with cyclohexane again, the samples were dried under a flow of nitrogen and marked on the backside (non-modified side). Copper (II) acetate ( $73 \mathrm{mg}, 0.40 \mathrm{mmol}$ ) was dissolved in water $(5 \mathrm{ml})$ in a $2.4 \times 7.5 \mathrm{~cm}$ ( $\varnothing \times$ height) vial, after which an aqueous solution of $30 \%$ hydrogen peroxide ( $15 \mathrm{ml}$ ) was added. Manual wetting of the cleaned PMMA was achieved by gently dipping it in the copper (II) acetate/hydrogen peroxide reaction mixture, after which the PMMA was placed (marking upwards) on the reaction mixture. The PMMA was gently pushed into (approximately $2 \mathrm{~cm}$ ) the reaction mixture by means of a syringe (SI Figure S1). Subsequent sonication $(37 \mathrm{kHz})$ of the mixture, while being allowed to warm up from room temperature to $50{ }^{\circ} \mathrm{C}$ over the course of $20 \mathrm{~min}$, ensured fluid homogeneity. The yellowish PMMA was then extensively rinsed with water and dried under a flow of nitrogen. It was thereafter placed (marking downwards) on the bottom of an ethylene glycol (anhydrous, $15 \mathrm{ml}$ ) filled $2.4 \times 7.5 \mathrm{~cm}$ ( $\varnothing \times$ height) vial, after which sodium borohydride flakes ( $100 \mathrm{mg}, 2.64 \mathrm{mmol}$ ) were added. This mixture was then sonicated $(37 \mathrm{kHz})$ at room temperature for $10 \mathrm{~min}$ and the, now colourless, PMMA was rinsed with water and sonicated $\left(37 \mathrm{kHz}, 30{ }^{\circ} \mathrm{C}, 10 \mathrm{~min}\right)$ in water in a reagent tube in which the samples were able to stand upright. Rinsing with water, drying under a flow of nitrogen followed by rinsing with cyclohexane and again drying under a flow of nitrogen afforded activated PMMA. Samples were either stored under argon (glovebox) for later use, stored in a vacuum oven (40 ${ }^{\circ} \mathrm{C}$ ) prior to XPS analysis, or used in the next reaction step immediately.

Silanisation of activated PMMA. In a glovebox, trichlorosilane (17.7 $\mu \mathrm{l}$ of a 0.99 M solution in anhydrous cyclohexane, $0.017 \mathrm{mmol}, 3.5 \mathrm{mM}$ final concentration) was dissolved in water-saturated cyclohexane $\left(5 \mathrm{ml}, 3.5 \mathrm{mM} \mathrm{H}_{2} \mathrm{O}\right.$ ) and gently shaken. One or two activated PMMA samples were placed (marking downwards) in the solution, after which the vials were tightly closed and taken out of the glovebox. After being wrapped with Parafilm ${ }^{\circledR}$, the vials were sonicated for 2 min and thereafter shaken at $110 \mathrm{rpm}$ for $60 \mathrm{~min}$. Cleaning of the samples was thereafter achieved by placing the samples (marking downwards) in fresh cyclohexane and subsequent shaking, after which the cyclohexane was replenished and the vial was shaken again (repeated twice). Rinsing with cyclohexane and drying under a stream of nitrogen afforded nanofilament-coated PMMA-polysiloxane hybrids (PMMA-Si-H).

Hydrosilylation of PMMA-Si-H. In a glovebox A, B, C or D $(0.45 \mathrm{mmol}, 1$ eq. $)$ and a solution of Karstedt's catalyst in vinyl-terminated poly(dimethylsiloxane) ( $2 \mu \mathrm{L}, 0.2 \mu \mathrm{mol}, 0.0004$ eq.) were dissolved in anhydrous $n$-hexane $(3 \mathrm{ml}$ ) and shaken thoroughly. One or two PMMA-Si-H samples were placed (marking downwards) in the solution, after which the vials were tightly closed and taken out of the glovebox. After being wrapped with Parafilm ${ }^{\circledR}$, the vials were incubated (without agitation) at 40 ${ }^{\circ} \mathrm{C}$ for $24 \mathrm{~h}$. Cleaning of the samples was thereafter achieved by placing the samples (marking downwards) in fresh cyclohexane and subsequent shaking, after which the cyclohexane was replenished and the vial was shaken again (repeated twice). Rinsing with cyclohexane and drying 
under a stream of nitrogen afforded fluorinated nanofilament-coated PMMA-polysiloxane hybrids (PMMA-Si-A to PMMA-Si-D).

\section{Hydrolytic stability}

A glass screw cap vial was filled with either neutral, acidic $(\mathrm{pH} 3$, acidified through addition of $1 \mathrm{M}$ $\mathrm{HCl}$ ) or basic ( $\mathrm{pH} 11$, basified through addition of $1 \mathrm{M} \mathrm{NaOH}$ ) water. A (fluorinated) PMMA-polysiloxane substrates was thereafter added to each of these vials and heated at $80^{\circ} \mathrm{C}$ for $24 \mathrm{~h}$. Afterwards, the substrates were taken out, thoroughly rinsed with water and dried over a stream of dry argon. Further drying was achieved by storing the surfaces in a vacuum oven for $16 \mathrm{~h}$. Some of the substrates used in this study were made by silanising under static conditions rather than through agitation at $110 \mathrm{rpm}$.

\section{Surface analysis}

Wetting behaviour. SWCA measurements were performed on a Krüss DSA 100 drop shape analyzer. SWCA's were assessed for both $3 \mu \mathrm{l}$ and $9 \mu \mathrm{l}$ water droplets, and the drop shape was fitted according to Tangent- 2 fitting parameters (Krüss DSA software version 1.90.0.14) by fitting at least 10× for each droplet. Reported values are an average of SWCA's measured over multiple droplets on a surface. Sliding angles were measured by depositing a $9 \mu \mathrm{l}$ water droplet on the surface, after which the surface was gradually tilted until the droplet fell off (reported values are averages over three or more measurements).

Transparency measurements. Visible light transmittance (transmittance\%, 400-800 nm, 600 scans $\mathrm{min}^{-1}$ ) was recorded on a Cary 50 scan UV-Visible Spectrophotometer (Varian) relative to the transmittance of ambient air. All reported values are averaged values of $\geq 2$ independent measurements (multiple spots on multiple surfaces).

Atomic force microscopy. AFM images were obtained with an MFP3D AFM (Asylum Research, Santa Barbara, CA). Imaging was performed in tapping mode in air. Images were flattened and the rootmean-square (RMS) roughness was calculated from the fluctuations of surface height. By doing so, the reported RMS values give an indication of surface roughness.

Scanning electron microscopy (SEM) imaging. Due to excessive charging of the polymeric samples, all surfaces were sputter-coated with gold $(60 \mathrm{sec}$, JFC-1300 (Jeol)) prior to imaging. Scanning electron microscopy (SEM) imaging was performed on a JAMP-9500F (J eol) Field Emission Auger Microscope with a primary beam energy of $5.0 \mathrm{kV}$ or $10.0 \mathrm{kV}$ at $5,000 \times$ and $10,000 \times$ magnification.

X-ray photoelectron spectroscopy (XPS). Core electron binding energies were measured by recording XPS spectra on a JPS-9200 photoelectron spectrometer (J EOL). Samples were irradiated at one or more spots with an Al Ka source emitting $1486.7 \mathrm{eV}$ photons, under a voltage of $12 \mathrm{kV}$ through which a current of $20 \mathrm{~mA}$ passed, while charge compensation was applied with an accelerating voltage of $2.8 \mathrm{eV}$ and a filament current of 4.80 A. Spectra were analysed using CasaXPS version 2.3.16 PR 1.6. The $C$ 1s peak emission was calibrated to a binding energy of $285.0 \mathrm{eV}$, and $\mathrm{C}$ is narrow scans were deconvoluted into their component peaks using Gaussian-Lorentzian sum functions having $30 \%-70 \%$ Gaussian-Lorentzian character. Due to surface irregularity (physically and chemically) no FWHM constraints were given when fitting peaks (except for bare PMMA).

Fourier transform-infrared (FTIR) spectroscopy. FTIR spectra were measured on a diamond crystal (Platinum ATR, Bruker) integrated in a Bruker Tensor 27 spectrophotometer system controlled through OPUS software (version 7.2.139.1294). All measurements were recorded with at least 16 scans and ambient air as background. 


\section{Quantum chemical calculations}

XPS $O$ 1s peak positions were calculated for the oxygen atoms of methyl acetate using B3LYP/6$311+G(d, p)$ according to a procedure described previously. ${ }^{[23 b]}$

\section{Acknowledgements}

Dr Rui Rijo Carvalho (Surfix Inc.) is thanked for his helpful comments and assistance. This project was funded by the NanoNextNL project 04A.01 "Biomimetic selective layers".

\section{References}

[1] a) T. Sun, L. Feng, X. Gao, L. Jiang, Acc. Chem. Res. 2005, 38, 644-652; b) P. Roach, N. J. Shirtcliffe, M. I. Newton, Soft Matter 2008, 4, 224-240; c) B. Bhushan, Y. C. Jung, Prog. Mater. Sci. 2011, 56, 1-108; d) S. Nishimoto, B. Bhushan, RSC Adv. 2013, 3, 671-690; e) B. Wang, W. Liang, Z. Guo, W. Liu, Chem. Soc. Rev. 2015, 44, 336-361; f) S. H. Li, J. Y. Huang, Z. Chen, G. Q. Chen, Y. K. Lai, J. Mater. Chem. A 2017, 5, 31-55; g) M. J. Liu, S. T. Wang, L. Jiang, Nat. Rev. Mater. 2017, 2, 17036; h) L. Feng, S. H. Li, Y. S. Li, H. J. Li, L. J. Zhang, J. Zhai, Y. L. Song, B. Q. Liu, L. Jiang, D. B. Zhu, Adv. Mater. 2002, 14, 1857-1860; i) G. R. J. Artus, S. Seeger, Adv. Colloid Interface Sci. 2014, 209, 144-162.

[2] J. Chapman, F. Regan, Adv. Eng. Mater. 2012, 14, B175-B184.

[3] L. L. Cao, A. K. Jones, V. K. Sikka, J. Z. Wu, D. Gao, Langmuir 2009, 25, 12444-12448.

[4] Y. Lai, Y. Tang, J. Gong, D. Gong, L. Chi, C. Lin, Z. Chen, J. Mater. Chem. 2012, 22, 74207426.

[5] A. Lafuma, D. Quere, Nat. Mater. 2003, 2, 457-460.

[6] E. G. Shafrin, W. A. Zisman in Contact Angle, Wettability, and Adhesion, Vol. 43, AMERICAN CHEMICAL SOCIETY, 1964, pp. 145-157.

[7] D. Quéré, Annu. Rev. Mater. Res. 2008, 38, 71-99.

[8] E. Fadeeva, V. K. Truong, M. Stiesch, B. N. Chichkov, R. J. Crawford, J. Wang, E. P. Ivanova, Langmuir 2011, 27, 3012-3019.

[9] J. U. Kim, S. Lee, T. I. Kim, J. Nanomater. 2016, 17.

[10] Y. Lu, S. Sathasivam, J. L. Song, C. R. Crick, C. J. Carmalt, I. P. Parkin, Science 2015, 347, $1132-1135$.

[11] C. K. Soz, E. Yilgor, I. Yilgor, Polymer 2015, 62, 118-128.

[12] Z. Geng, J. H. He, J. Mater. Chem. A 2014, 2, 16601-16607.

[13] K. Ellinas, S. P. Pujari, D. A. Dragatogiannis, C. A. Charitidis, A. Tserepi, H. Zuilhof, E. Gogolides, ACS Appl. Mater. Interfaces 2014, 6, 6510-6524.

[14] a) S. Jung, G. Artus, J. Zimmermann, S. Seeger (University Of Zurich), WO2004113456, 2004; b) G. R. J. Artus, S. Jung, J. Zimmermann, H. P. Gautschi, K. Marquardt, S. Seeger, Adv. Mater. 2006, 18, 2758-2762; c) E. Kasapgil, E. G. Atici, R. Cicek, I. Anac, H. Y. Erbil, RSC AdV. 2016, 6, 74921-74928.

[15] L. Bardos, H. Barankova, Thin Solid Films 2010, 518, 6705-6713.

[16] R. R. Carvalho, S. P. Pujari, E. X. Vrouwe, H. Zuilhof, ACS Appl. Mater. Interfaces 2017, 9, 16644-16650.

[17] a) D. A. Markov, E. M. Lillie, S. P. Garbett, L. J. McCawley, Biomed. Microdevices 2014, 16, 91-96; b) M. Rosso, V. van Steijn, L. C. P. M. de Smet, E. J. R. Sudholter, C. R. Kleijn, M. T. Kreutzer, Appl. Phys. Lett. 2011, 98, 174102.

[18] A. Torikai, M. Ohno, K. Fueki, J. Appl. Polym. Sci. 1990, 41, 1023-1032.

[19] J. Zhang, S. Seeger, Angew. Chem. Int. Ed. 2011, 50, 6652-6656. 
[20] a) J. Guo, W. Fang, A. Welle, W. Feng, I. Filpponen, O. J. Rojas, P. A. Levkin, ACS Appl. Mater. Interfaces 2016, 8, 34115-34122; b) J. Li, L. Li, X. Du, W. Feng, A. Welle, O. Trapp, M. Grunze, M. Hirtz, P. A. Levkin, Nano Lett. 2015, 15, 675-681.

[21] D. E. Rollings, J. G. C. Veinot, Langmuir 2008, 24, 13653-13662.

[22] P. Louette, F. Bodino, J.-J. Pireaux, Surf. Sci. Spectra 2005, 12, 69-73.

[23] a) M. Giesbers, A. T. M. Marcelis, H. Zuilhof, Langmuir 2013, 29, 4782-4788; b) J. Zhao, F. Gao, S. P. Pujari, H. Zuilhof, A. V. Teplyakov, Langmuir 2017, 33, 10792-10799.

[24] a) J. B. Brzoska, I. Benazouz, F. Rondelez, Langmuir 1994, 10, 4367-4373; b) E. P. Plueddemann, Silane coupling agents, 2nd ed., Plenum Press, New York, 1991.

[25] J. W. Roddy, C. F. Coleman, Talanta 1968, 15, 1281-1286.

[26] K. C. Khulbe, C. Y. Feng, T. Matsuura, Synthetic Polymeric Membranes: Characterization by Atomic Force Microscopy, Springer Berlin, 2007.

[27] G. R. J. Artus, L. Bigler, S. Seeger, Langmuir 2014, 30, 10308-10316.

[28] C. B. Walsh, E. I. Franses, Thin Solid Films 2003, 429, 71-76.

[29] S. P. Pujari, E. Spruijt, M. A. Cohen Stuart, C. J. M. van Rijn, J. M. J. Paulusse, H. Zuilhof, Langmuir 2012, 28, 17690-17700. 


\section{Supporting information corresponding to Chapter 5}

Figure S1. Set-up for copper-catalysed oxidation for activation of PMMA.

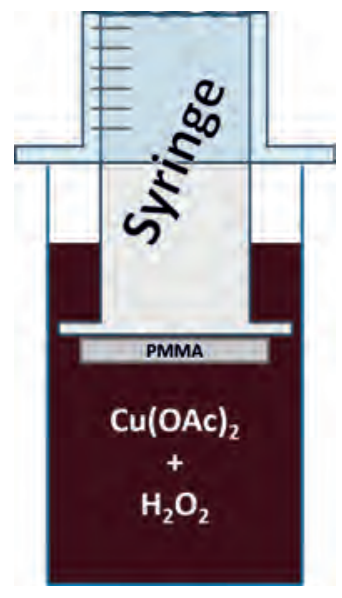

Figure S2. AFM analysis of bare PMMA.

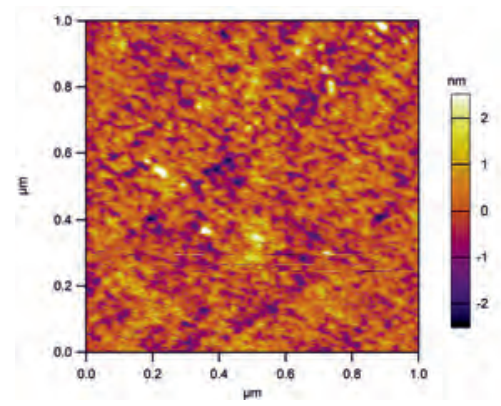

A: Height topography.

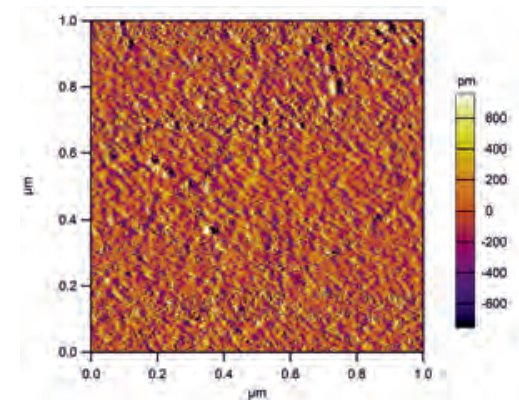

B: Amplitude topography.

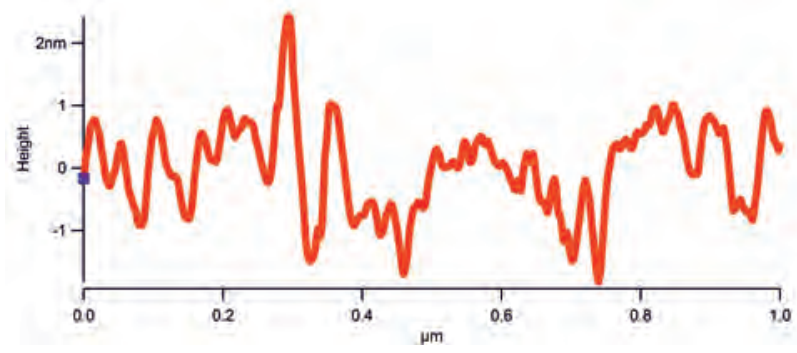

C: Roughness profile indicating an average roughness of $0.6 \mathrm{~nm}$. 
Figure S3. AFM analysis of activated PMMA.

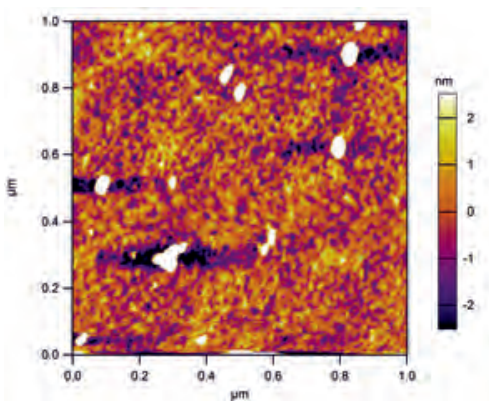

A: Height topography.

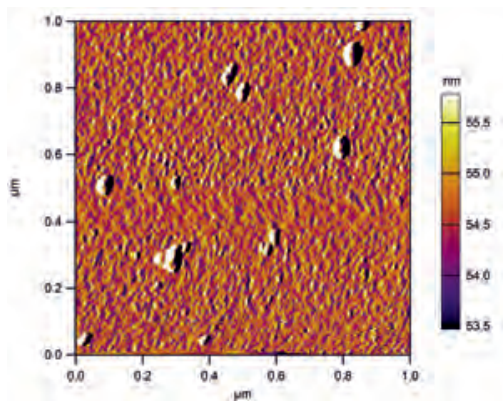

B: Amplitude topography.

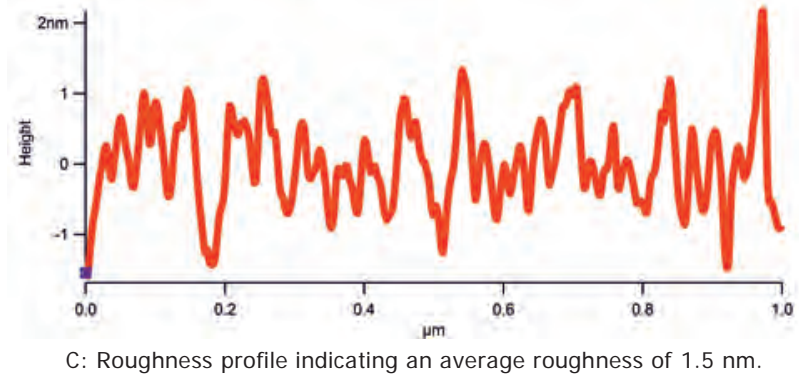

Figure S4A. XPS analysis of bare PMMA; wide scan.

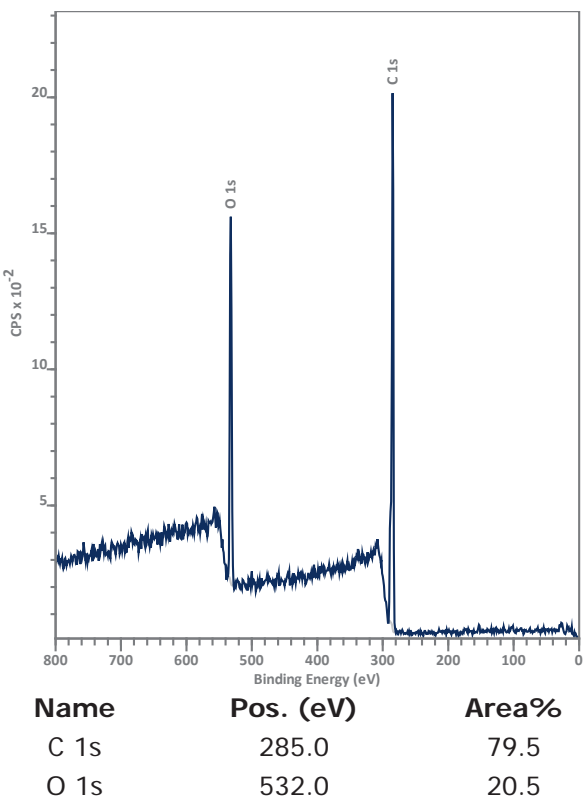

Figure S4B. XPS analysis of bare PMMA; $C$ 1s narrow scan.

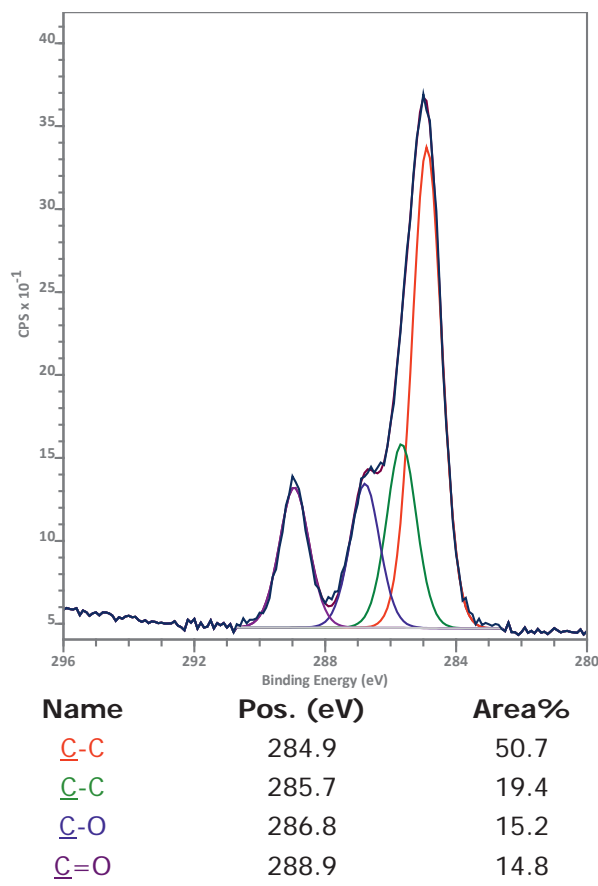


Figure S4C. XPS analysis of bare PMMA; 0 1s narrow scan.

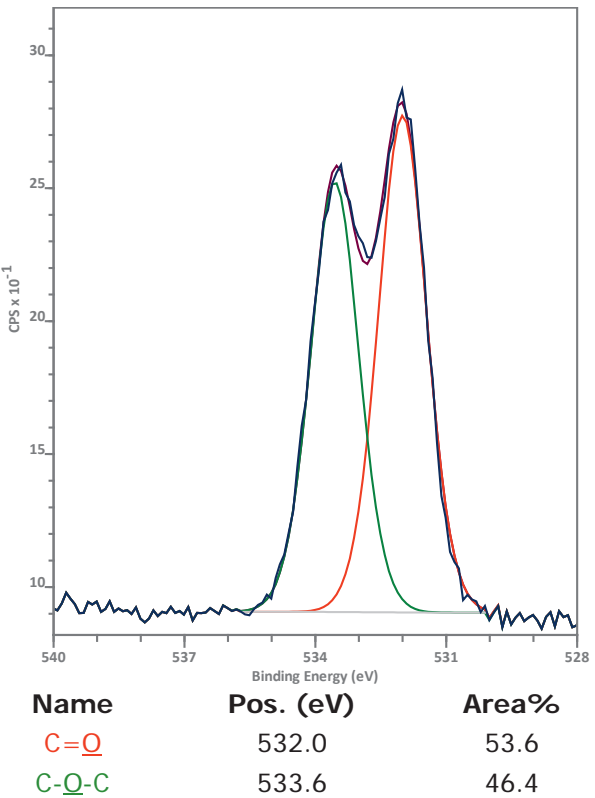

Figure S5B. XPS analysis of activated PMMA; C 1s narrow scan.

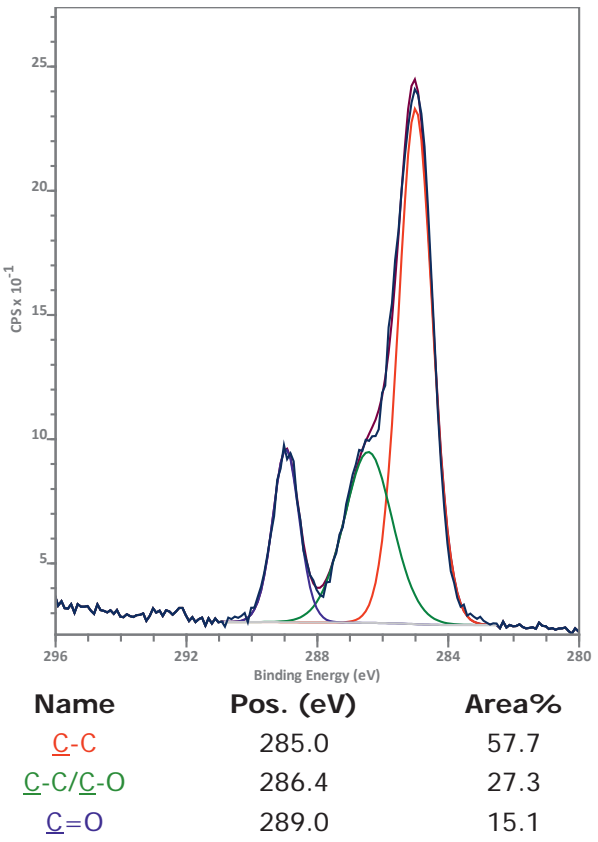

Figure S5A. XPS analysis of activated PMMA; wide scan.

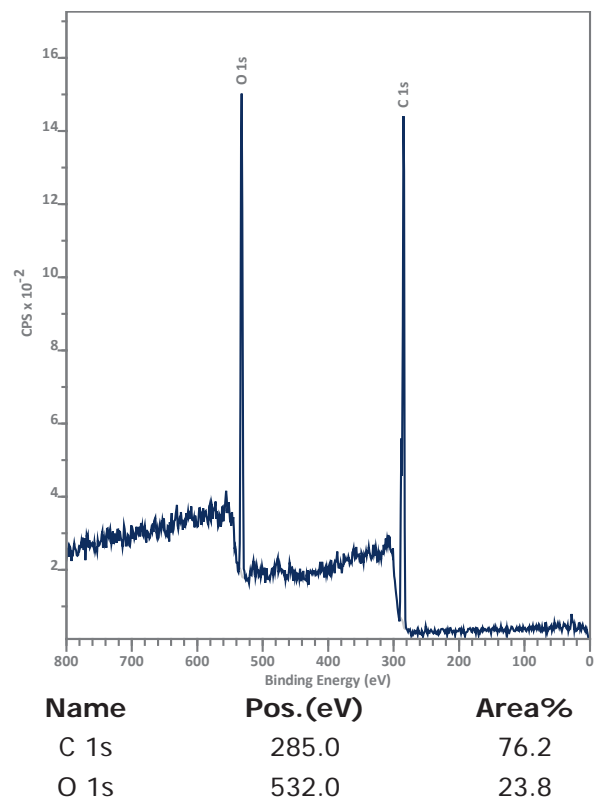

Figure S5C. XPS analysis of activated PMMA; 0 is narrow scan.

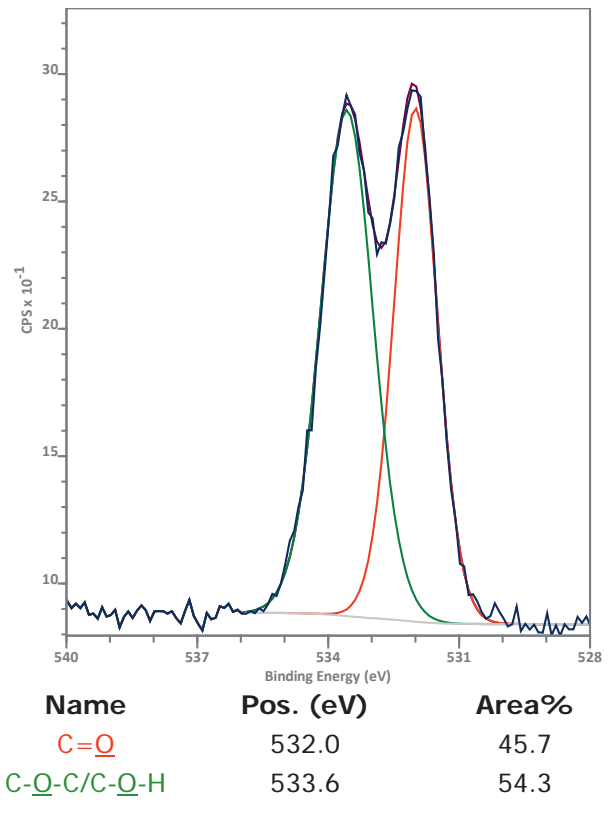


Figure S6A. XPS analysis of PMMA-Si-H; wide scan.

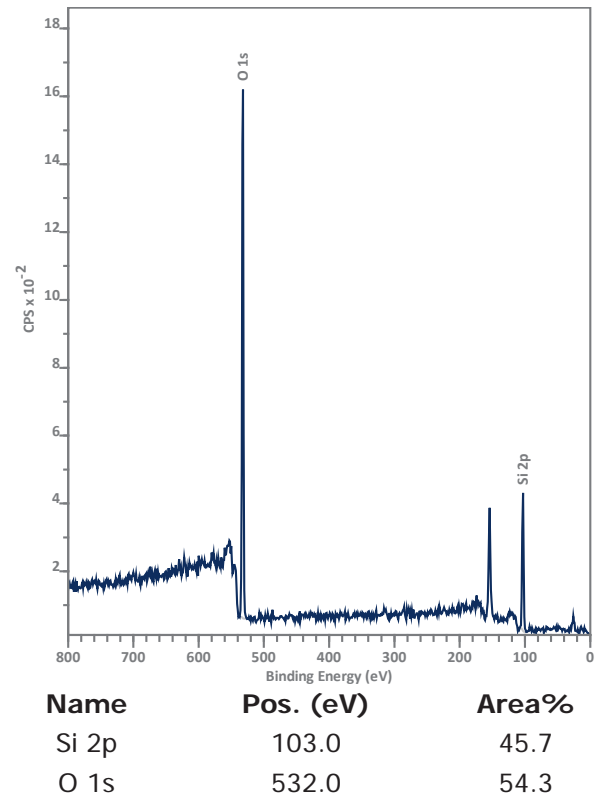

Figure S6C. XPS analysis of PMMA-Si-H; 0 Is narrow scan.

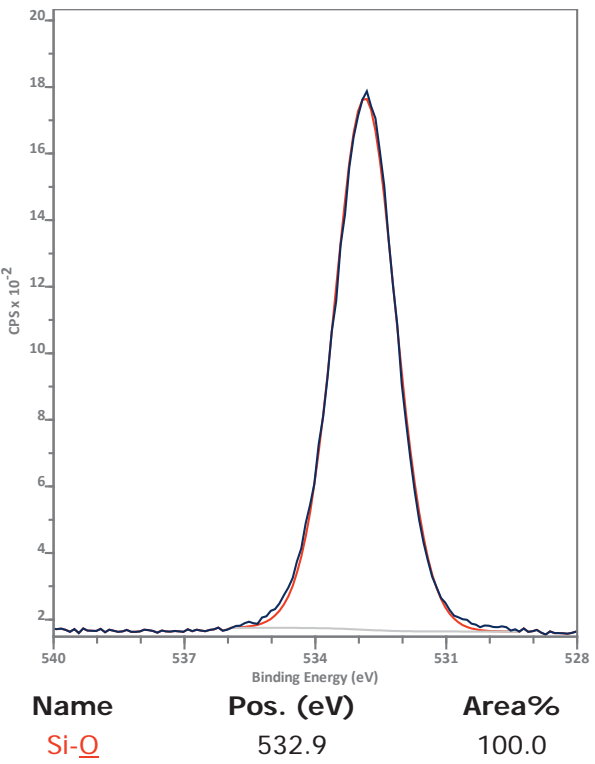

Figure S6B. XPS analysis of PMMA-Si-H; $C$ is narrow scan.

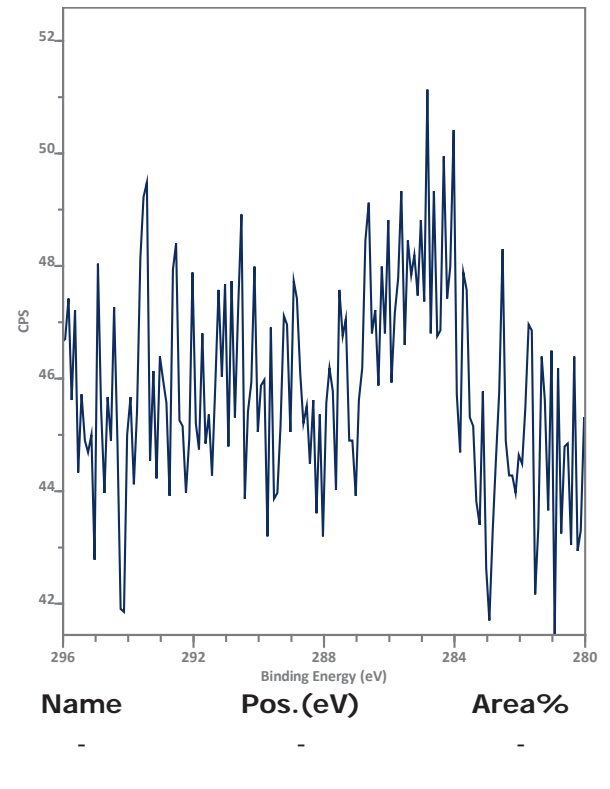

Figure S6D. XPS analysis of PMMA-Si-H; Si $2 p$ narrow scan.

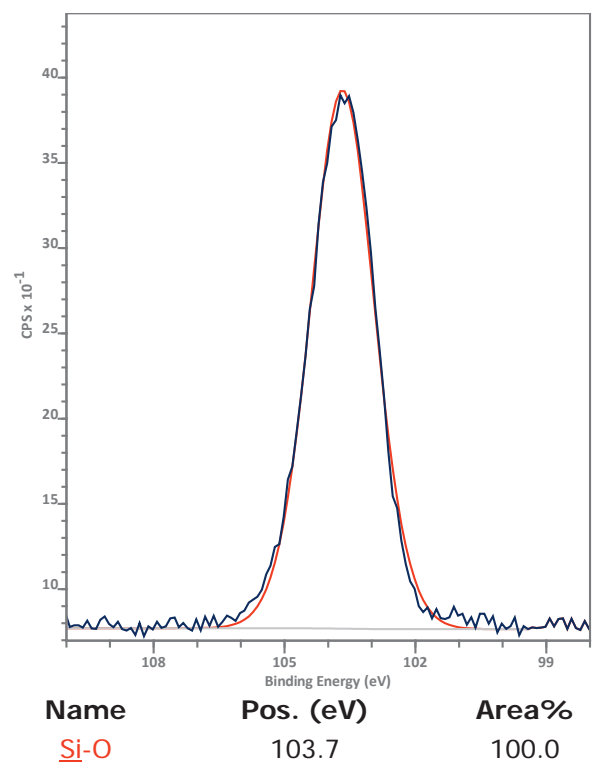


Figure S7A. XPS analysis of PMMA-Si-A; wide scan.

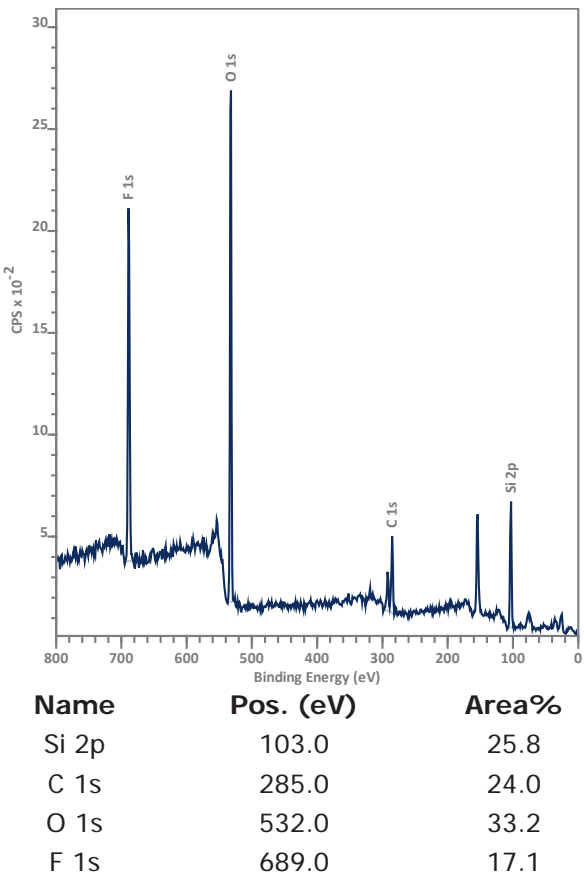

Figure S7C. XPS analysis of PMMA-Si-A; 0 is narrow scan.

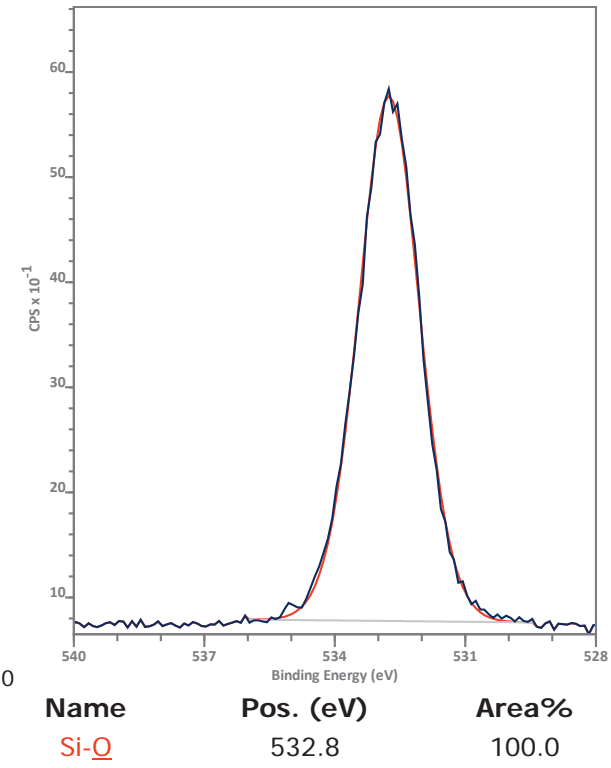

Figure S7B. XPS analysis of PMMA-Si-A; $C$ is narrow scan.

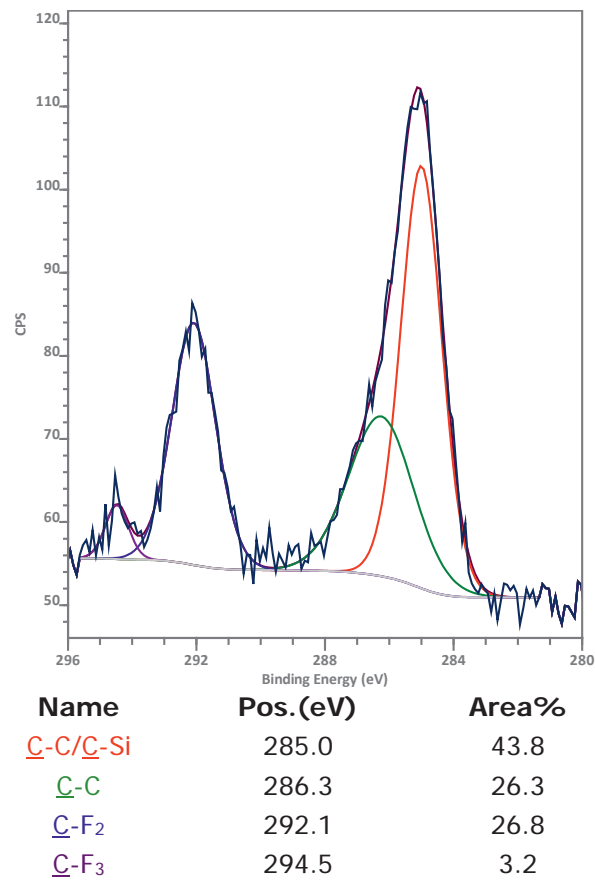

Figure S7D. XPS analysis of PMMA-Si-A; Si $2 p$ narrow scan.

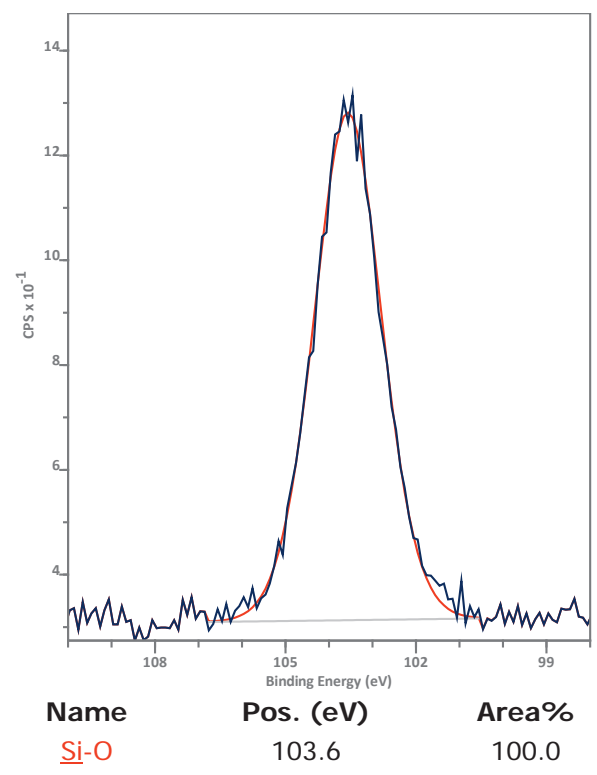


Figure S7E. XPS analysis of PMMA-Si-A; F 1s scan.

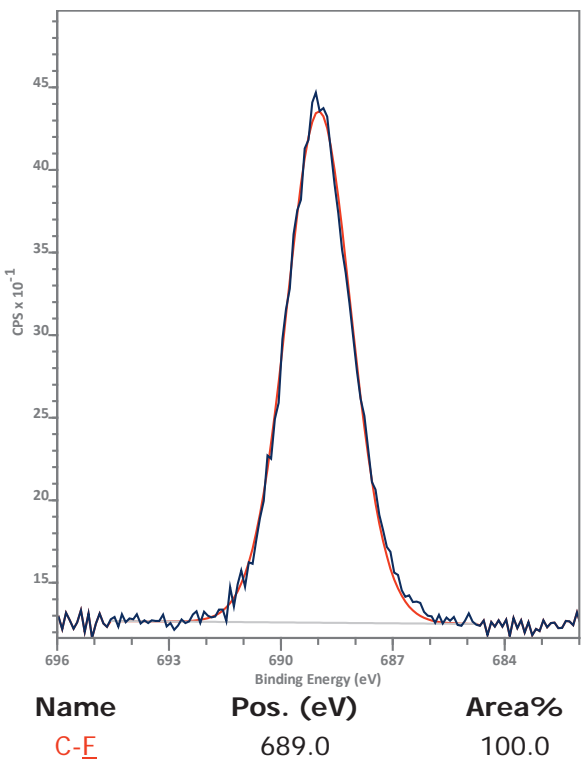

Figure S8B. XPS analysis of PMMA-Si-B; $C$ 1s narrow scan.

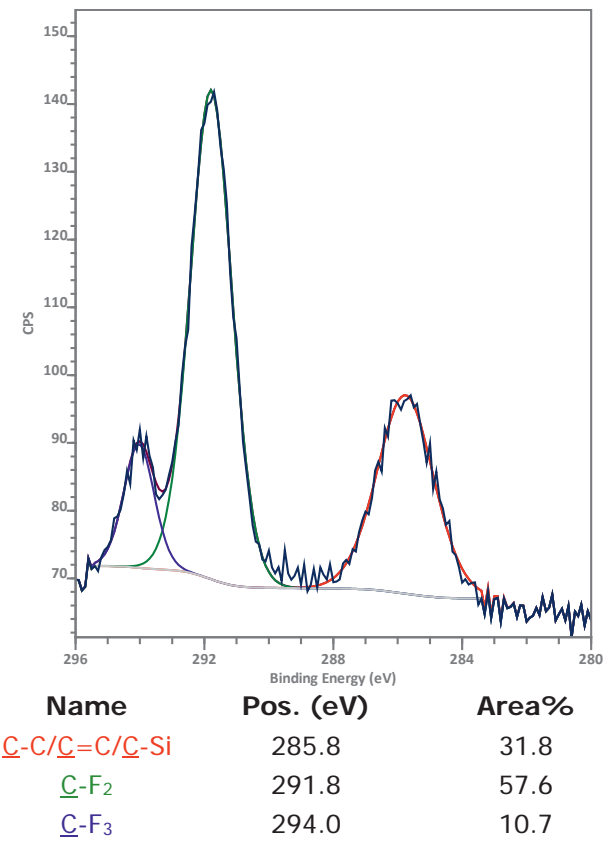

Figure S8A. XPS analysis of PMMA-Si-B; wide scan.

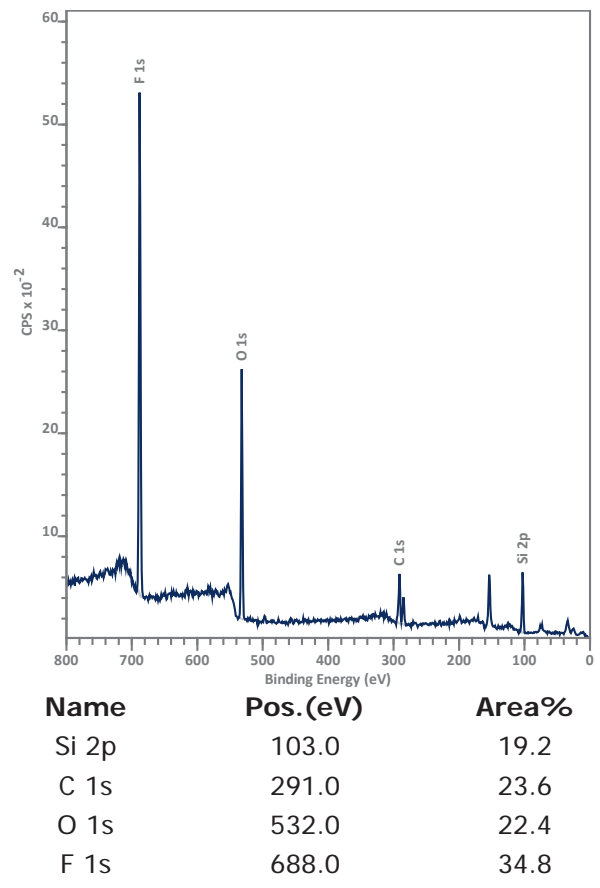

Figure S8C. XPS analysis of PMMA-Si-B; $O$ Is narrow scan.

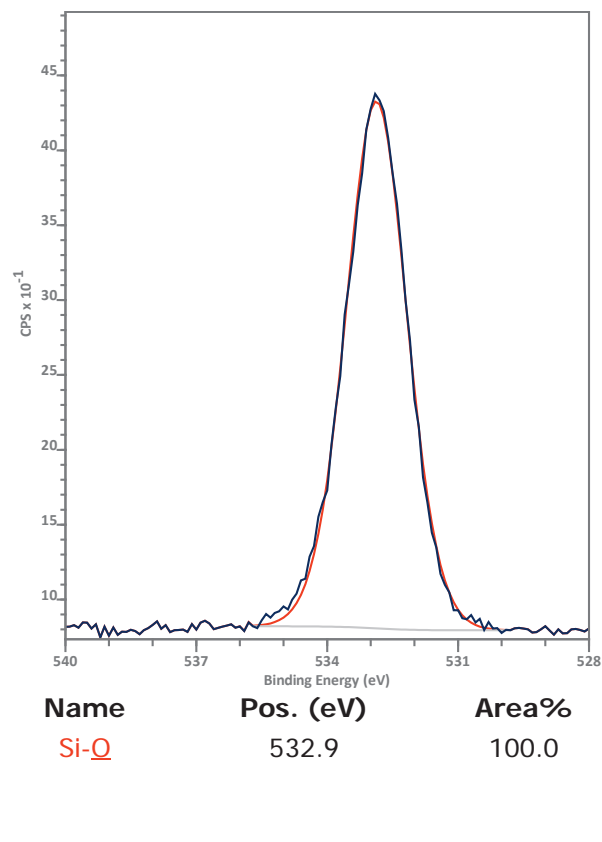


Figure S8D. XPS analysis of PMMA-Si-B; Si $2 p$ narrow scan.

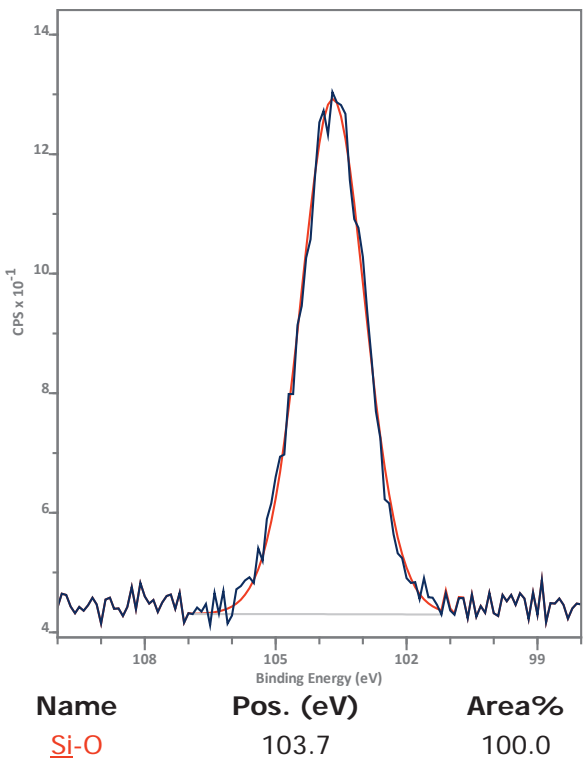

Figure S9A. XPS analysis of PMMA-Si-C; wide scan.

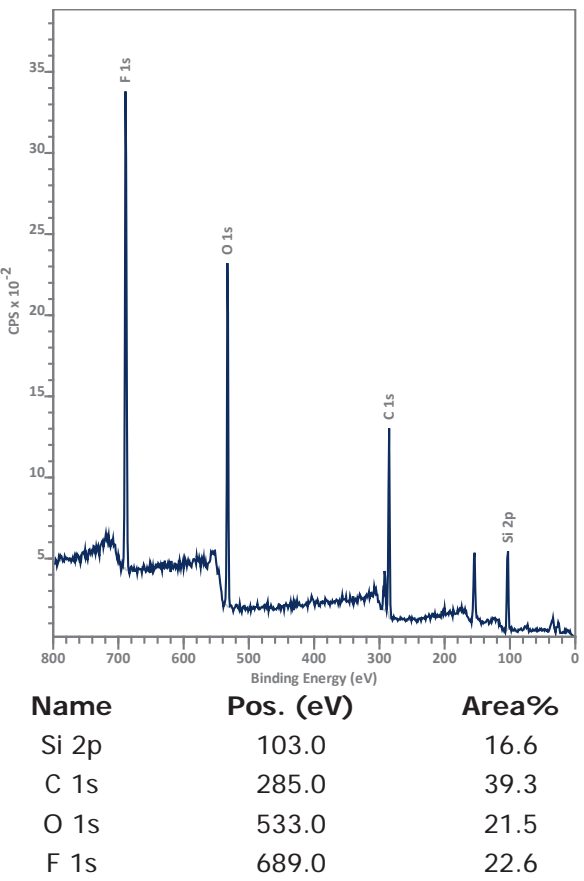

Figure S8E. XPS analysis of PMMA-Si-B; $F$ Is narrow scan.

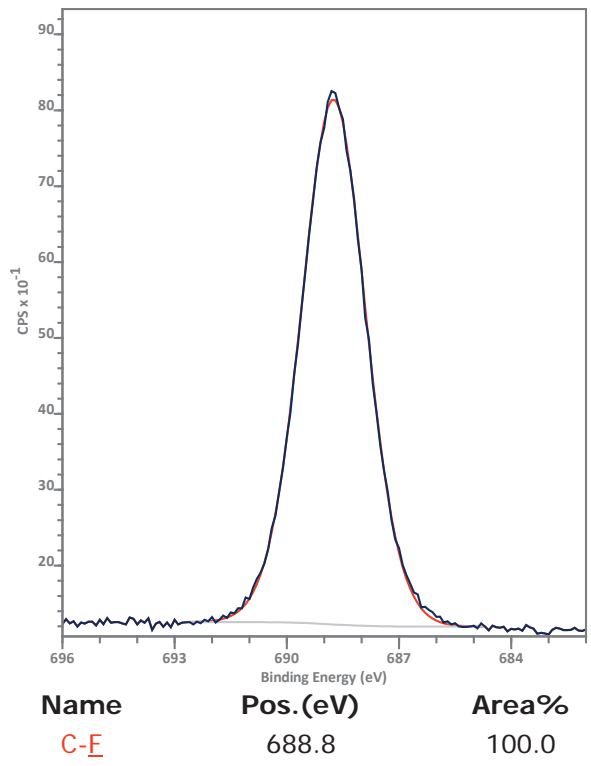

Figure S9B. XPS analysis of PMMA-Si-C; $C$ ls narrow scan.

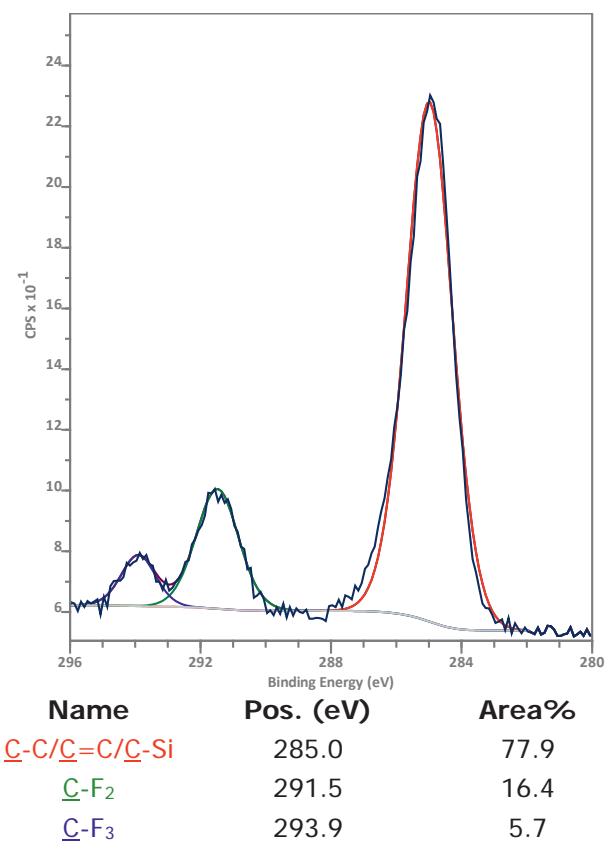


Figure S9C. XPS analysis of PMMA-Si-C; 0 1s narrow scan.

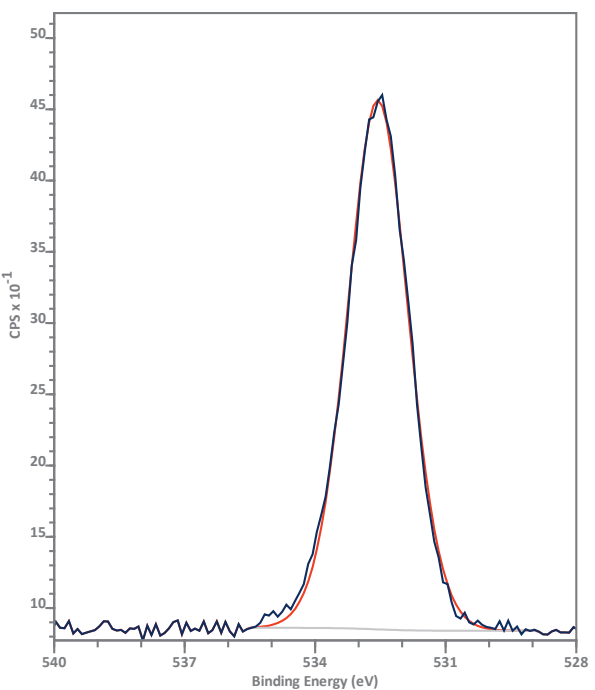

$$
\begin{array}{ccc}
\text { Name } & \text { Pos. (eV) } & \text { Area\% } \\
\text { Si- } \underline{0} & 532.6 & 100.0
\end{array}
$$

Figure S9E. XPS analysis of PMMA-Si-C; $F$ 1s narrow scan.

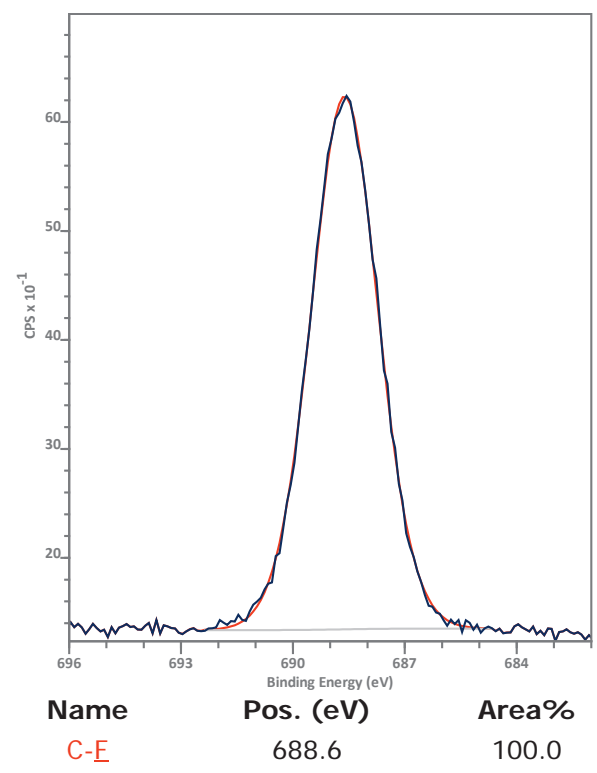

Figure S9D. XPS analysis of PMMA-Si-C; Si $2 p$ narrow scan.

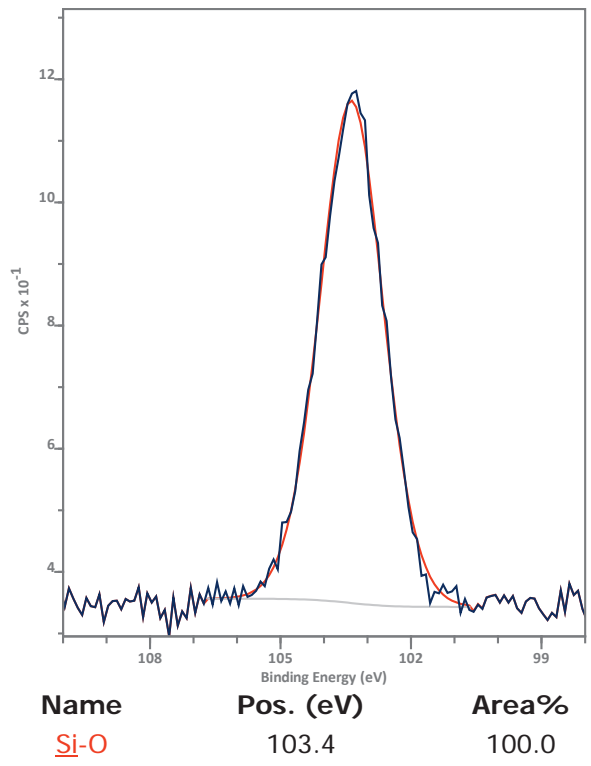

Figure S10. SEM image of PMMA-Si-A (10 kV, 10.000x).

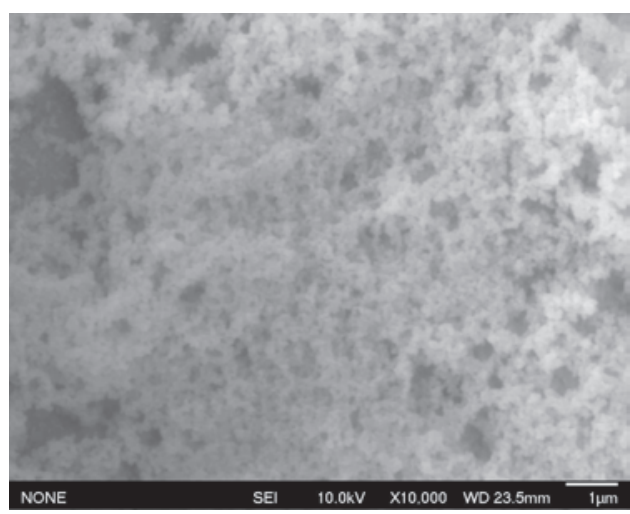


Figure S11. XPS wide scans of PMMA-polysiloxane hybrids after being heated in water of $\mathrm{pH} 3\left(80^{\circ} \mathrm{C}, 24 \mathrm{~h}\right)$.

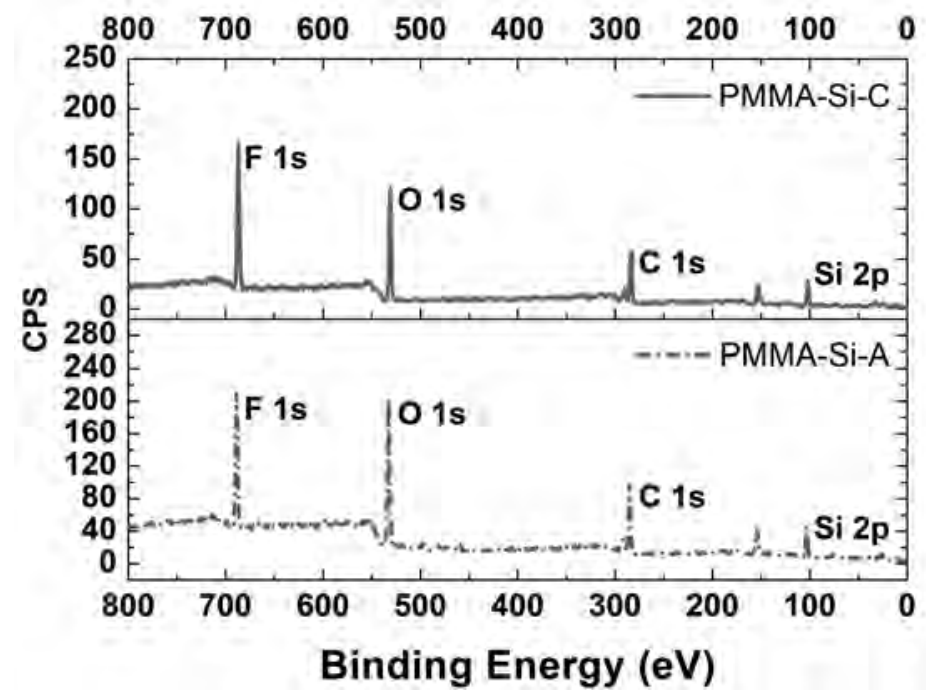

\begin{tabular}{lllll} 
& \multicolumn{4}{c}{ XPS atomic percentage } \\
& C 1s & F 1s & Si 2p & O 1s \\
PMMA-Si-C & 40.7 & 20.6 & 16.0 & 22.6 \\
PMMA-Si-A & 36.4 & 17.6 & 19.3 & 26.8
\end{tabular}

Figure S12. XPS wide scans of PMMA-polysiloxane hybrids after being heated in neutral water $\left(80^{\circ} \mathrm{C}, 24 \mathrm{~h}\right)$.

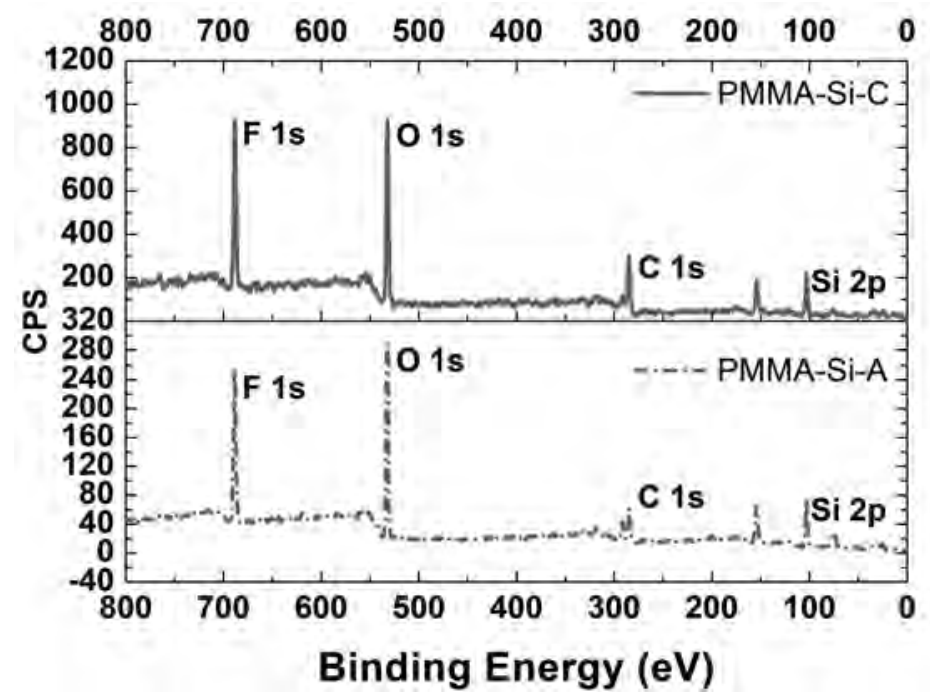

\begin{tabular}{lllll} 
& \multicolumn{5}{c}{ XPS atomic percentage } \\
& C 1s & F 1s & Si 2p & O 1s \\
PMMA-Si-C & 40.7 & 20.6 & 16.0 & 22.6 \\
PMMA-Si-A & 36.4 & 17.6 & 19.3 & 26.8
\end{tabular}


Figure S13. XPS wide scans of PMMA-polysiloxane hybrids after being heated in water of pH $11\left(80^{\circ} \mathrm{C}, 24 \mathrm{~h}\right)$.

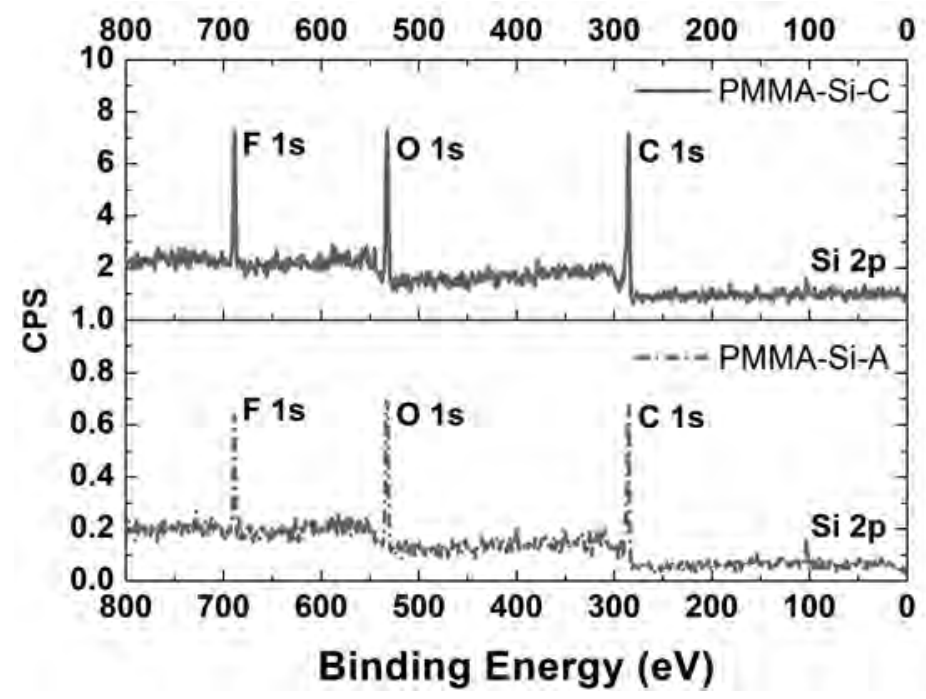

\begin{tabular}{lllll} 
& \multicolumn{5}{c}{ XPS atomic percentage } \\
& C 1s & F 1s & Si 2p & O 1s \\
PMMA-Si-C & 40.7 & 20.6 & 16.0 & 22.6 \\
PMMA-Si-A & 36.4 & 17.6 & 19.3 & 26.8
\end{tabular}

Figure S14. SEM images of left: PMMA-Si-A and right: PMMA-Si-C after being heated in water of $\mathrm{pH} 3\left(80^{\circ} \mathrm{C}, 24 \mathrm{~h}\right)$.
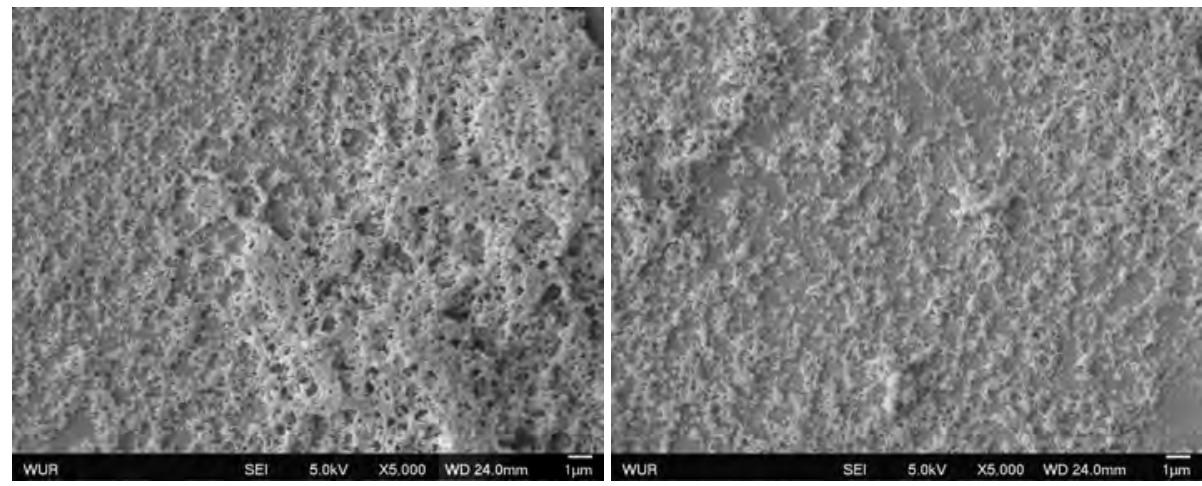
Figure S15. SEM images of left: PMMA-Si-A and right: PMMA-Si-C after being heated in neutral water $\left(80^{\circ} \mathrm{C}, 24 \mathrm{~h}\right)$.
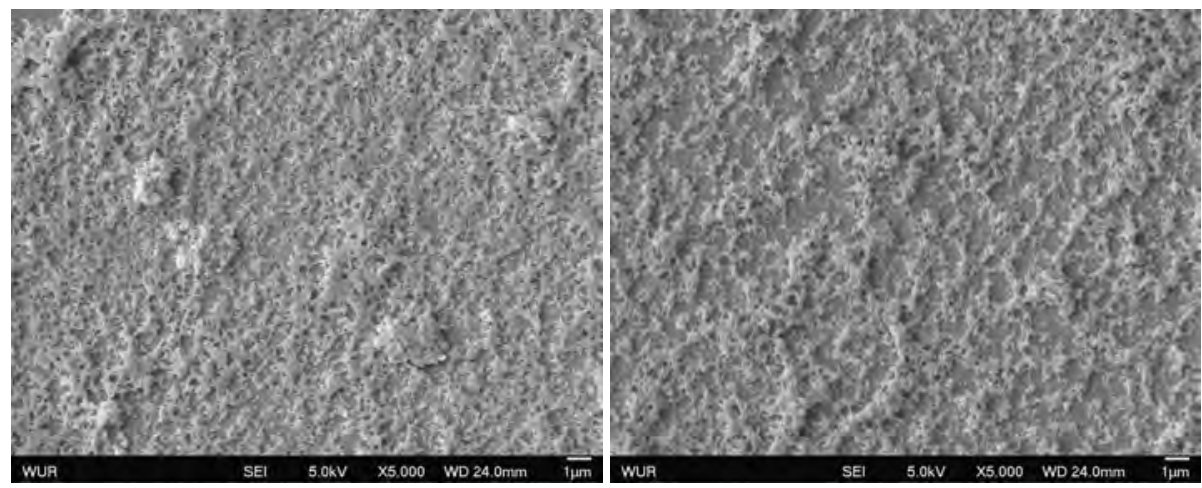

Figure S16. SEM images of left: PMMA-Si-A and right: PMMA-Si-C after being heated in water of $\mathrm{pH} 11\left(80^{\circ} \mathrm{C}, 24 \mathrm{~h}\right)$.

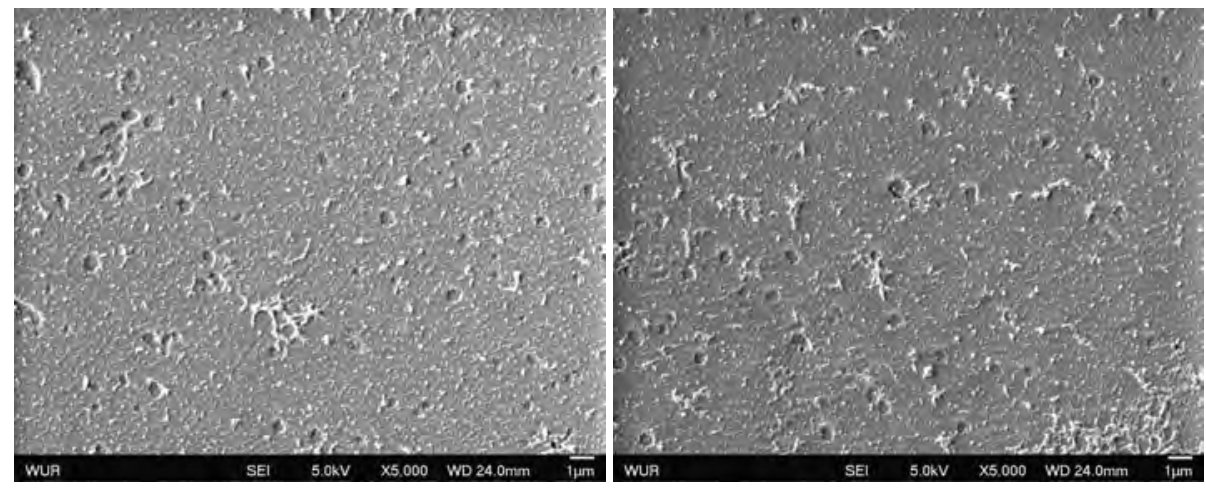




\section{Chapter 6}

\section{General discussion}

* This chapter is directly followed by its corresponding supporting information. 
Previous chapters outlined the applicability of various strategies for the mild covalent functionalisation of synthetic polymers. The current chapter will summarise the highlights of earlier chapters, and discuss remaining challenges, suggestions and provide an outlook on future research.

\section{Chapter 2. Characterisation of the laccase-mediated oligomerisation of 4-hydroxybenzoic acid}

Laccase-mediated oligomerisation of 4-hydroxybenzoic acid (4-HBA) and concomitant in-situ modification of poly(ethersulfone) (PES) membranes proved to be a powerful strategy for inducing anti-fouling properties to these membranes. In order to comprehend the mechanism behind this functionalisation strategy, solution-phase oligomerisation had to be understood first. Chapter 2 therefore describes how laccase converts 4-HBA; this process proceeds only slowly ( $26 \%$ conversion over $24 \mathrm{~h}$ ), initially mainly resulting in the formation of C3-C3' and C3-O-bound dimers. Subsequent laccase-mediated conversion of dimers proved to be far more rapid. This was supported by the fact that the energy required for the oxidation of the neutral species to the radical cation proved far lower for these dimers than for 4-HBA itself, as was determined through quantum chemical calculations. Due to rapid subsequent oligomerisation, occurring through both $\mathrm{C}-\mathrm{O}$ and $\mathrm{C}-\mathrm{C}$ bond formation, product diversity proved to be large.

\section{Outlook}

As dimers that were less abundant in the reaction mixture were also converted more rapidly, we concluded that dimer reactivity was the main factor governing their low abundance. In order to fully comprehend dimer behaviour, the influence of their formation on dimer abundance, rather than only their subsequent oligomerisation, should be studied too. Quantum chemical calculations, as those performed in Chapter 2, only probed radical cation formation by the enzyme. It was previously reported that the spin density of the 4-HBA radical is mainly located on the phenolic oxygen atom. ${ }^{[1]}$ In order to find out to what degree this prevalence contributes to dimer formation, a complete reaction profile for dimer formation should be outlined. Both the energy required for binding two 4-HBA radicals (termination) and for binding a 4-HBA radical with neutral 4-HBA (propagation) followed by abstraction of a proton radical (termination) should therefore be calculated. For the formation of the $\mathrm{C} 1-\mathrm{C}^{\prime}$ ' and $\mathrm{Cl}$-O dimer (Scheme 1), this involves an additional transition state subsequent to monomer coupling, in which decarboxylation has not taken place yet. Obtaining the complete reaction profile for the formation of dimers should also clarify why the C1-O dimer could not (or barely) be observed after laccase-mediated conversion of 4-HBA, while being less reactive than the distinctly present $\mathrm{Cl}-\mathrm{C}^{\prime}$ dimer.

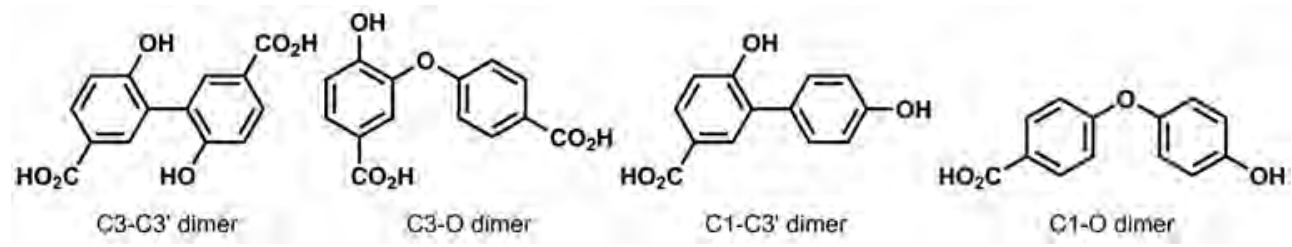

Scheme 1. Dimers of 4-hydroxybenzoic acid.

It was furthermore suggested that radical cation formation is followed by rapid loss of a proton to produce the respective radical species. Using pulse radiolysis, the decay of the radical cation to the radical species has indeed been shown to be extremely rapid for phenols bearing electron-withdrawing substituents (radical cation lifetime < $500 \mathrm{~ns}$ ). ${ }^{[2]}$ However, the exact behaviour of the 4-HBA radical cation that had been formed from 4-HBA as solely present oxidisable species at $\mathrm{pH} 5$ has, to the best of our knowledge, not been studied yet. ${ }^{[3]}$ The role of the phenol radical cation is of particular interest 
as it was recently suggested that laccase-mediated trifluoromethylation occurs through a reaction with a phenol radical cation rather than through a reaction with the respective radical species. ${ }^{[4]}$

Diaryl ethers are ubiquitous sub-structures found in many different natural products, including hormones and antibiotics. ${ }^{[5]}$ One of these natural products is Aristogin C (Scheme 2), a constituent of Aristolochia elegans, ${ }^{[6]}$ which is in fact a methylated derivative of the C3-O dimer. Our use (Chapter 2) of an arylboronic acid as aryl donor in the, so-called, Chan-Lam coupling has allowed for the straightforward single step synthesis of this natural product. This strategy afforded Aristogin C without requiring additional solid-phase couplings, as was essential for synthetic approaches published earlier, while being equally efficient ( $49 \%$ vs $55 \%$ yield). ${ }^{[7]}$ Hence, Chan-Lam coupling is seen as the solution to a long-standing problem in diaryl ether synthesis. ${ }^{[8]}$ As Aristogin F (Scheme 2) also bears a carboxylic acid moiety and Aristogin D is the partially demethylated variant of Aristogin $C_{,}{ }^{[9]}$ we anticipate also the $\mathrm{C} 3-\mathrm{O}$ dimer to be unambiguously identified as natural product in future research.

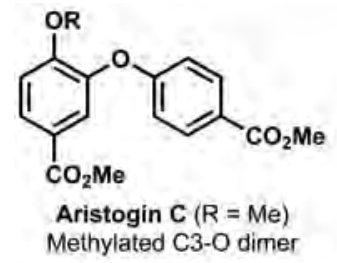

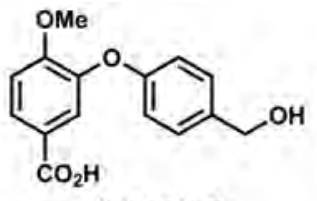

Aristogin F<smiles>O=C(O)c1ccc(Oc2cc(C(=O)O)ccc2O)cc1</smiles>

Aristogin G? C3-O dimer

Aristogin $D(R=H)$

Scheme 2. Natural products from the Aristogin family and the C3-O dimer as possible member of this family.

In order to probe its applicability, it is worthwhile to study whether Chan-Lam coupling would also allow for easy access to the rest of the Aristogin family and other diaryl ether natural products. Aristogin $\mathrm{F}$ could, for example, be obtained from the Chan-Lam coupling of 4-formylphenylboronic acid and methyl 3-hydroxy-4-methoxybenzoate followed by reduction of the aldehyde moiety and hydrolysis of the methyl ester. Analogously, Chan-Lam coupling of commercially available building blocks 4-(2-methoxycarbonylethyl)phenylboronic acid and methyl (E)-3-(3-hydroxy-4methoxyphenyl)acrylate followed by (acid-free) demethylation of the methoxy group would grant easy access to Spiraformin A (Scheme 3), a natural product isolated from Spiraea formosana that has been employed in traditional Chinese medicine to treat a wide variety of diseases. ${ }^{[10]}$ It can be expected that this two-step synthesis would allow for an improvement over the $25 \%$ overall yield of the current synthetic procedure. ${ }^{[7]}$

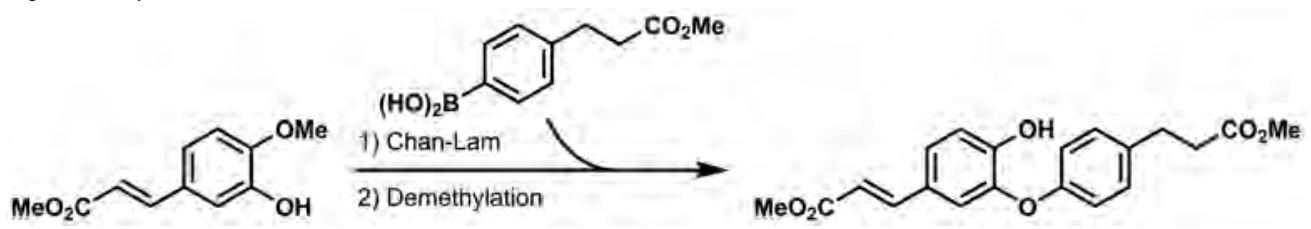

Scheme 3. Synthetic strategy towards Spiraformin A.

Curine and tubocurine are derivatives of tubocurarine (the main active component of arrow-poison curare) and have only recently been synthesised for the first time. ${ }^{[11]}$ Ullmann coupling afforded the cyclised product in only $17 \%$ yield (including MOM ether deprotection) after 12 days at $125-130{ }^{\circ} \mathrm{C}$ (Scheme 4). Commonly, Chan-Lam coupling does not require high temperatures and might therefore be a more favourable route towards these alkaloids. Prior to Chan-Lam coupling a boronic acid or boronate should be installed by starting from the brominated precursor (Cur.Br). This could be achieved through traditional metal-catalysed Miyaura borylation or through a recently published highyielding metal-free photochemical borylation, ${ }^{[12]}$ which has proven to be tolerant to a wide range of functional groups. Subsequent intramolecular $\mathrm{Cu}(\mathrm{OAC})_{2}$-mediated Chan-Lam coupling and debenzylation would thereafter furnish $( \pm)$-curine and $( \pm)$-tubocurine. 
<smiles>COc1ccc(C[C@H]2c3c(cc(OC)c(OCc4ccccc4)c3Br)CCN2C)cc1Oc1ccc(Oc2cc3c(cc2OC)CCN(C)[C@H]3Cc2ccccc2)cc1</smiles>

1) Borylation

2) MOM ether deprotection
1) MOM ether deprotection 2) Ullmann cyclisation ( $17 \%, 2$ steps)

3) Debenzylation

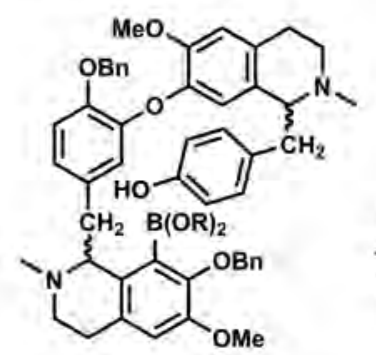

$\mathrm{R}=\mathrm{H}$ or alkyl (Cur.B)

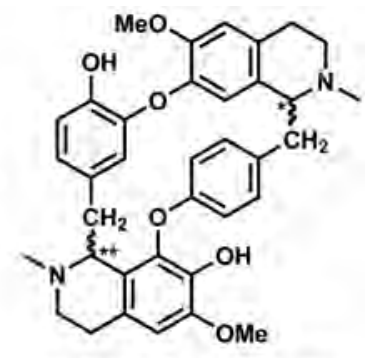

$*=S, *=R( \pm)$-tubocurine

$*=R,{ }^{*}=R(t)$-curine

1) Chan-Lam cyclisation

2) Debenzylation

Scheme 4. Synthetic routes towards $( \pm)$-curine and $( \pm)$-tubocurine. Upper route: according to literature ${ }^{[11]}$ and lower route: proposed herein.

\section{Chapter 3. Elucidating the mechanism behind the laccase- mediated modification of poly(ethersulfone)}

Apart from functionalising poly(ethersulfone) (PES) with oligomers of 4-HBA, we envisioned that in-situ laccase-mediated oxidation of other phenolics would also lead to membrane modification. However, the use of phenols bearing positively charged substituents did not (or barely) result in surface functionalisation. In an attempt to determine the binding modes between 4-HBA (oligomers) and PES, we incubated laccase and 4-HBA in the presence of PES model compounds. Covalent bond formation between 4-HBA (dimers or oligomers) and the model compounds could not be observed. Attempts at the in-situ laccase-mediated functionalisation of PES using soluble negatively charged phenolic oligomers also proved unsuccessful, and extensive washing of membranes modified with oligomeric 4-HBA led to loss of colouration. It was therefore concluded that laccase-mediated functionalisation of PES resulted from strong physical adsorption of coloured oligomeric phenols, rather than from covalent bond formation with those.

\section{Remarks}

Where laccase-mediated surface functionalisation of PES using soluble phenolics could not be achieved, doing so using other phenols (i.e. 4-hydroxybenzoic acid, gallic acid) did prove to result in a relatively stable coating (no discolouration after agitation in water of $40{ }^{\circ} \mathrm{C}$ for $72 \mathrm{~h}$ ). Prolonged use of modified PES in systems where it is subjected to flow (i.e. membrane reactors and microfluidics instruments) is, however, not advisable as parts of the coating might leach into the medium. One should additionally be careful with using solvents/media that are either (strongly) acidic or basic. The solubility of the coating will be enhanced in basic media in those cases where the applied coating is, for example, rich in carboxylic acid and/or hydroxy moieties. This will likewise hold for coatings rich in amine moieties and their use in acidic media. ${ }^{[13]}$

In order for any future application, based on the laccase-mediated conversion of phenols bearing positively charged substituents, to be successful, further optimisation of the reaction conditions for the conversion of A-F (Scheme 5) has to be achieved. Apart from tuning the usual parameters, such as temperature, $\mathrm{pH}$ and concentration, specific care has to be taken concerning the choice of the counterion. For the research presented in Chapter 3 the counterion iodide was replaced by chloride, as 
iodide has been shown to also be converted by laccase and could therefore act as a competitive inhibitor or lead to unwanted iodination. ${ }^{[14]}$ Chloride itself, however, is also not an innocent counterion as it was shown to substantially inhibit the laccase-catalysed conversion of an anthraquinone dye, even at 50 mM. ${ }^{[15]}$ For small-scale experiments, as those performed in Chapter 3, this could easily be circumvented by using more laccase. For future applications in which the use of costly enzyme is the limiting factor however, iodide could be exchanged for formate or acetate.<smiles>C[N+](C)(C)CCc1ccc(O)cc1</smiles>

A<smiles>COC(=O)c1cc(C[N+](C)(C)C)c(O)c(C[N+](C)(C)C[N+](C)(C)Cc2cc(OC)cc(C[N+](C)(C)C)c2O)c1</smiles>

B
C

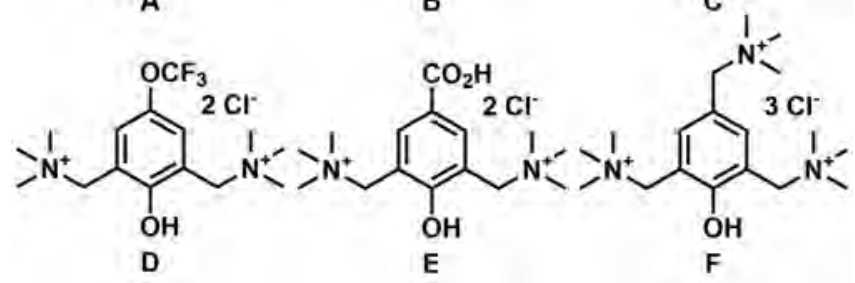

Scheme 5. Positively charged phenols A-F.

\section{Outlook}

Analogous to the laccase-mediated polymerisation of syringic acid, ${ }^{[16]}$ (co-)polymerisation of $\mathbf{E}$ would provide easy access to highly positively charged poly(phenylene oxides). Poly(phenylene oxides) are of special interest due to their high mechanical and chemical stability. ${ }^{[17]}$ Poly(phenylene oxide) diversification is now commonly performed through laborious post-production modifications of poly(2,6-dimethyl-1,4-phenylene oxide), ${ }^{[18]}$ whereas laccase-mediated polymerisation of E would offer a more eco-friendly alternative. These positively charged polymers of $\mathbf{E}$ could find applications as anion exchange membrane, ${ }^{[19]}$ which is of specific interest due to a growing need for these polymers in fuel cell applications for example.

Even though laccase-mediated grafting on poly(ethersulfone) could not be established, the use of this enzyme to covalently functionalise other materials with charged phenolics might prove valuable as they are likely to convey anti-bacterial properties to the material. ${ }^{[20]}$ Current laccase-based strategies for conveying anti-bacterial properties to lignin heavily rely on the immobilisation of simple phenols. ${ }^{[21]}$ These molecules, however, are not per se excellent anti-microbials, where positively charged quaternary ammonium salts (i.e. A-F) are likely to be highly potent anti-bacterial agents. ${ }^{[22]}$ Additionally, as was elaborated on in Chapter 4, laccase-mediated grafting of simple (mostly hydrophobic) phenols concomitantly results in the formation of poorly soluble homopolymers that subsequently adsorb to the surface and are difficult to remove, but leach out over time. As charged phenols B-F remain highly soluble upon oligomerisation, laccase-mediated wood surface functionalisation with these phenols is likely to only result in grafting and not adsorption.

The work discussed in Chapter 4 has also shown that current attempts at improving the antibacterial properties of chitosan through laccase-mediated surface functionalisation with simple phenols has little added value due to the anti-bacterial properties of chitosan itself. However, functionalisation with phenols bearing a quaternary ammonium salt substituent is expected to enhance the antibacterial properties of this material even further. ${ }^{[23]}$ Where covalent laccase-mediated functionalisation of PES could not be achieved, chitosan might be susceptible to modification using laccase. As is stated in Chapter 4, Michael addition of the formed quinone to chitosan's amine group, or imine formation 
from the amine and quinone, are the most common pathways for laccase-mediated chitosan functionalisation. The use of either of the phenolics $\mathbf{B}-\mathbf{F}$ is therefore unlikely to result in functionalisation of chitosan. Formation of a quinone, subsequent to laccase-mediated hydroxylation, is hampered due to the presence of substituents on the ortho- and para-positions of their phenol ring. However, the use of phenol $\mathbf{A}$ to achieve chitosan modification should be viable as its C3 and C5 positions are unsubstituted. Unfortunately, as outlined in Chapter 3, laccase-mediated oligomerisation of $\mathbf{A}$ has proven to result in the release of trimethylamine with concomitant formation of, most likely, 4-vinylphenol derivatives, which could be embedded in the formed oligomers and thereby decrease the solubility of these oligomers resulting in their precipitation. Alternatively, methylation of the dimethylamine group of commercially available 2-((dimethylamino)methyl)phenol (Scheme 6: 1) could provide easy access to a positively charged phenol that does not bear additional substituents. Furthermore, as was shown in Chapter 3, laccase-mediated conversion of phenols bearing a $N, N, N$ trimethylmethanaminium substituent (i.e. B-F), rather than a $N, N, N$-trimethylethan-1-aminium substituent (i.e. A), did not lead to the release of trimethylamine. Laccase-mediated conversion of methylated 2-((dimethylamino)methyl)phenol $\left(\mathbf{1}^{+}\right)$would thus lead to the formation of the respective ortho-quinone and paraquinone, which are susceptible to covalent binding with chitosan. Laccasemediated oligomerisation of charged phenolic monomers that are unsubstituted at the para- and ortho-position(s) would additionally result in crosslink-formation leading to an increase in charge density. Alternatively, the presence of a second tertiary amine (8) would allow for access to a related doubly charged phenol $\left(\mathbf{8}^{2+}\right)$.
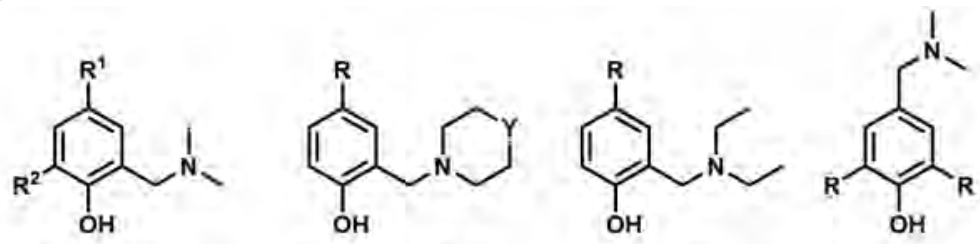

$$
\begin{aligned}
& R^{1}=R^{2}=H(1) \\
& R^{1}=R^{2}=M e(2) \\
& R^{1}=M e, R^{2}=t B u(3) \\
& R^{1}=C H O, R^{2}=H(4) \\
& R^{1}=C H O, R^{2}=\text { OMe (5) }
\end{aligned}
$$

$$
\begin{aligned}
& \mathrm{R}=\mathrm{H}, \mathrm{Y}=\mathrm{CH}_{2}(6) \\
& \mathrm{R}=\mathrm{H}, \mathrm{Y}=\mathrm{O}(\mathbf{7}) \\
& \mathrm{R}=\mathrm{H}, \mathrm{Y}=\mathrm{NMe}(\mathbf{8}) \\
& \mathrm{R}=\mathrm{Me}, \mathrm{Y}=\mathrm{CH}_{2}(9) \\
& \mathrm{R}=\mathrm{CO}_{2} \mathrm{H}, \mathrm{Y}=\mathrm{O}(10)
\end{aligned}
$$

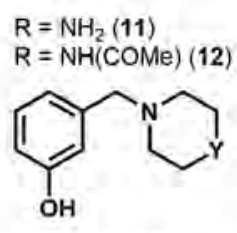

$$
\mathrm{Y}=\mathrm{CH}_{2}(15)
$$$$
\mathrm{Y}=\mathrm{NH}(16)
$$$$
\mathrm{Y}=\mathrm{CHNH}_{2}(17)
$$

$$
R=\operatorname{Pr}(13)
$$

$R=t B u(14)$

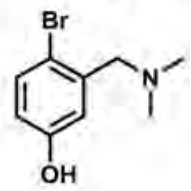

18

Scheme 6. Commercially available (dialkylamino)methyl-substituted phenols $\left(<150 € \mathrm{~g}^{-1}\right)$ (1-18) of which quaternisation of their tertiary amine groups would lead to positively charged phenols $\left(\mathbf{1}^{+}-\mathbf{1 8}^{+}\right)$as interesting targets for use in laccase-mediated grafting.

2-((Dimethylamino)methyl)phenol is only one of various commercially available (dialkylamino)methyl-substituted phenols (Scheme 6: 1-18, SI Scheme S1: 19-25), which, together with the ease by which this substituent can be introduced using the Mannich reaction, gives access to a rich library of these compounds. Subsequent quaternisation of their tertiary amine substituent(s) subsequently gives rise to a large arsenal of compounds conceivably possessing anti-bacterial properties. Laccase-mediated surface functionalisation (of lignin for example) using these phenols could convey this property to the material while simultaneously installing a reactive handle to allow for subsequent chemistries.

The use of lignin/chitosan composites as adsorbent material has recently gained considerable attention, as it allows for the valorisation of lignin waste. ${ }^{[24]}$ These composites are likely to be further strengthened by crosslinking both materials. The use of quaternised aldehyde derivatives $\mathbf{4}^{+}$or $\mathbf{5}^{+}$ could aid in doing so, by first grafting it on lignin by means of laccase, after which chitosan is added to 
result in imine bond formation between the aldehyde-functionalised crosslinker and the amine groups present in chitosan (Scheme 7). The crosslinker will additionally provide anti-bacterial properties to the material they are grafted on, which makes its use especially interesting for applications in which this material encounters (biologically) contaminated effluents, e.g. in drinking water purification.

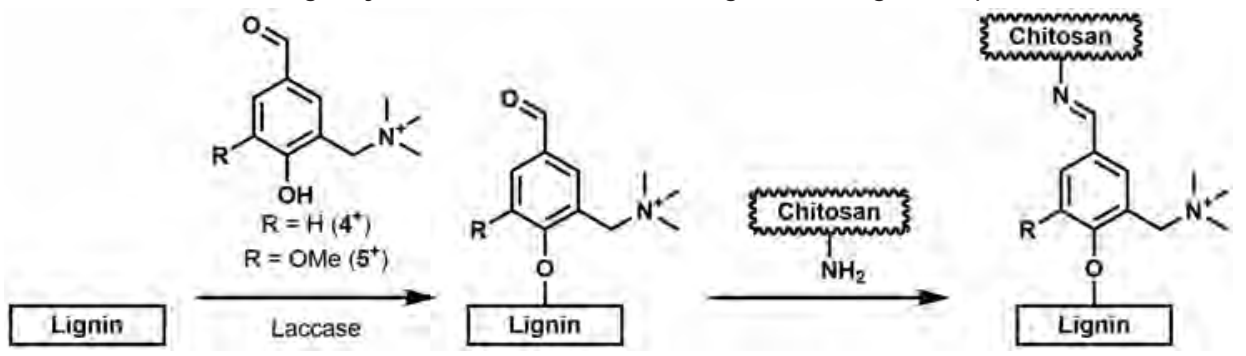

Scheme 7. The use of conceivably anti-bacterial $\mathbf{4}^{+}$or $\mathbf{5}^{+}$as crosslinker for chitosan and lignin.

Laccase-mediated surface functionalisation using $\mathbf{1 3}^{+}$or $\mathbf{1 4}^{+}$would offer an alternative to the use of $\mathbf{A}$ as conversion of $\mathbf{1 3}^{+}$or $\mathbf{1 4 ^ { + }}$ would not lead to deamination, oligomerisation and precipitation. In order to obtain reasonable turnovers of especially $\mathbf{1 4}^{+}$, one is, however, restricted to the use of mediators due to the sterically congested hydroxy substituent. ${ }^{[25]}$ The presence of a (protected) primary $(\mathbf{1 1}, \mathbf{1 2}$ or 17), secondary amine (16) or bromine substituent (18) would allow for further modification of the (dialkylamino)methyl-substituted phenol scaffold prior, or subsequent, to quaternisation and/or grafting and would thereby give rise to almost endless functionalisation chemistries.

Socha and co-workers have used a reductive amination strategy to convert lignin-derived aldehyde-substituted phenols to non-permanently positively charged aminium ion-substituted phenols. Their dihydrogen phosphate salts were elegantly employed as ionic liquids in biomass (lignin) pretreatment. ${ }^{[26]}$ In a recent patent, these authors have also suggested to use permanently positively charged quaternary amines for this purpose. ${ }^{[27]}$ This might thus open up a completely new application area for our (multiply) charged phenol derivatives (A-F). Additionally, abundant commercial availability of these Mannich bases (1-18 and 19-25) and easy access to others through a Mannich reaction as employed by us, would allow alternative access to a rich library of potential ionic liquids.

It was recently shown that laccase could also be used in Atom Transfer Radical Polymerisation (ATRP) reactions instead of small-molecule copper catalysts. By employing a reducing agent (ascorbate), laccase-mediated acrylate polymerisation could be performed in the absence of air or oxygen. ${ }^{[28]}$ In this elegant approach, one of laccase's copper sites is reduced by ascorbate rather than through internal electron transfer. By doing so, controlled living polymerisation of several acrylic and vinylic monomers could be initiated from (soluble) alkyl halides. Ko and co-workers used this approach to graft acrylate monomers from alkyl halide-functionalised lignin. ${ }^{[29]}$ As for all controlled living polymerisations, all traces of oxygen should be completely abolished or require a cumbersome workaround in order to tolerate oxygen, which increases complexity and handling time. Additionally, the required presence of alkyl halide initiators (on the surface) and high concentrations of laccase shows that the applicability of oxygen-free laccase-mediated acrylate polymerisation is only limited.

Over the last decade, grafted zwitterionic polymer brushes have proven to possess excellent antifouling properties, ${ }^{\left[{ }^{[3]}\right.}$ far superior over their scientific predecessors: poly(ethylene glycol)s. ${ }^{[31]}$ These brushes are commonly synthesised from zwitterionic methacrylate monomers whose polymerisation is initiated from a surface-bound bromo-initiator. ${ }^{[32]}$ Laccase-initiated grafting of $N$-(3-sulfopropyl)- $N$ (methacryloxyethyl)- $N, N$-dimethylammonium betaine, a sulfobetaine methacrylate, was shown to be possible, but resulted in only minor surface coverage. ${ }^{[33]}$ As was discussed by Hollmann and coworkers, ${ }^{[34]}$ ineffective polymerisation was most likely caused by the presence of oxygen that acted as radical scavenger. As laccase-mediated polymerisation of syringic acid has already proven to be a 
viable way of generating high molecular weight polymers at room temperature in the presence of oxygen, ${ }^{[16]}$ laccase-mediated surface functionalisation with a zwitterionic 4-hydroxybenzoic acid derivative (Scheme 8) should grant easy access to ultralow fouling materials (i.e. wood or paper). These zwitterion-substituted phenols should be easily obtained via the (dimethylamino)methylsubstituted precursor E3 (Scheme 8). Rather than quaternising the tertiary amine group through methylation, one is likely to obtain carboxybetaines by employing lactones or halide-substituted alkyl carboxylate salts (Scheme 9). Alternatively, sulfobetaines could be obtained by reacting a sultone or halide-substituted alkyl sulfonate salt together with the (dialkylamino)methyl-substituted phenol in a single step.<smiles>COC(=O)c1cc(COC(=O)c2cc(CN(C)C)c(O)c(C[N+](C)(C)CCS(=O)(=O)[O-])c2)c(O)c(CN(C)C)c1</smiles>

Scheme 8. Zwitterionic monomers derived from 4-hydroxybenzoic acid (similar to E) and a possible precursor; E3.

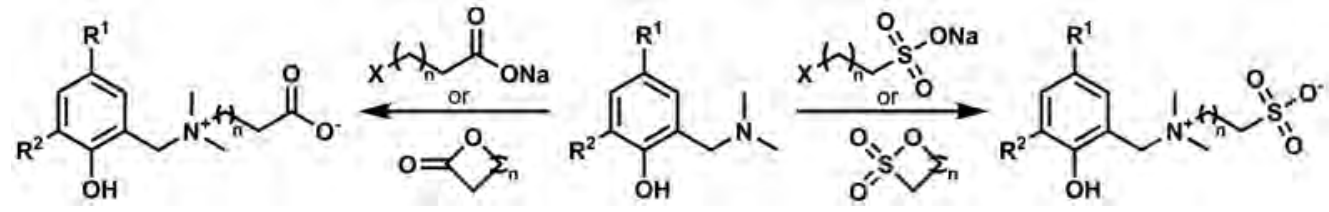

Scheme 9. Potential single step routes towards zwitterionic phenols.

Apart from allowing isolation of (conjugated) PES model compounds from the reaction mixture, we also stated that a resin-bound PES model compound would mimic the PES surface to a greater extent than small-molecule PES model compounds would do. This statement is, however, only partially true, as the resin that the PES model is bound to, is able to swell while binding the PES model compound via a long flexible linker. In order to mimic the polymer surface better, a non-swelling matrix, which binds the (PES) model compound through a short and/or rigid linker, should be used. A silicon matrix would provide these features while also being highly ordered and allowing for dense functionalisation. ${ }^{[35]} \mathrm{A}$ short Wang-like linker should be easily obtained by etherification of protected 4hydroxybenzyl alcohol with propargyl bromide (Scheme 10). Subsequent photochemical hydrosilylation ${ }^{[36]}$ on etched silicon particles or porous silicon would provide a silicon matrix which resembles Wang resins. This silicon-based Wang-like matrix can thereafter be functionalised with a PES model compound and be used as a PES surface mimic in laccase-mediated modification reactions. Analogous to the use of Wang resins, reacted or unreacted PES model compounds can thereafter be cleaved with diluted trifluoroacetic acid.

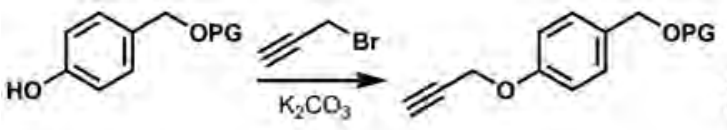
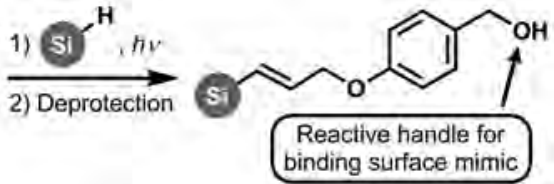

Scheme 10. Synthesis of a silicon-based Wang-like matrix as support for studying surface modification reactions.

The use of resin-bound model compounds to mimic a material's exterior could prove to be a very convenient tool for also studying other systems like laccase-mediated grafting on lignin. ${ }^{[37]}$ Transition metal-catalysed valorisation of lignocellulosic biomass has proven to be a hot topic, but selective transformations to achieve this are commonly conducted on small-molecule model compounds. ${ }^{[38]}$ The use of lignin model compounds immobilised on a silicon-based Wang-like matrix as proposed above might also aid in the characterisation and improvement of these non-enzymatic transformations. 
Another tool that has proven to be highly valuable in determining the mechanism of the laccasemediated functionalisation of poly(ethersulfone) was the use of negatively charged phenols. These commercially available negatively charged phenols remained highly soluble upon oligomerisation and therefore did not precipitate and could easily be washed off the membrane. If these phenols or their respective oligomers would have been observed on the surface after laccase-mediated modification, this would thus indicate functionalisation via a different route than adsorption: covalent grafting. As was described in Chapter 4, many laccase-mediated surface functionalisation strategies rely on the oligomerisation of poorly soluble phenols. Where this is often likely to result in subsequent grafting of these phenols and their oligomers (on lignin for example), mere adsorption of the poorly soluble oligomers is also to be expected. Replacing the intended target phenol with a phenol having a similar oxidation potential that remains soluble upon oligomerisation (i.e. guaiacol sulfonate) to see whether its use also results in surface functionalisation can thereby indicate if, and to what degree, laccasemediated surface modification of the target phenol is the result of covalent grafting.

\section{Chapter 4. Laccase-mediated grafting on biopolymers and synthetic polymers: a critical review}

As was described in Chapter 3 , it proved to be highly challenging to elucidate the mechanism behind the laccase-mediated functionalisation of PES surfaces. An arsenal of complementary techniques was required to be able to unequivocally state that PES modification was caused by adsorption of phenolic oligomers rather than by grafting of those. These results prompted us to review laccase-mediated surface modification strategies published recently and assess the likelihood of grafting. Our judgement was based on five factors: mechanistic rationale, pre-treatment, control experiments, washing/cleaning and the used analytical tools. It could be concluded that in multiple cases laccase-mediated grafting would have been improbable or contributed only minimally to surface functionalisation.

\section{Novel research on laccase-mediated grafting}

Even though, at the time of writing of this chapter, the literature review of Chapter 4 had been published only approximately half a year ago, quite a number of interesting papers have since been published on the topic of laccase-mediated grafting. Some selected examples will be discussed in this paragraph.

As a continuation of their earlier work, ${ }^{[37 b]}$ Kabel and co-workers have shown that mediators might not only bind to lignin model compounds, but thereby also influence their subsequent reactions. ${ }^{[39]}$ Where laccase converted HOBt only slowly, ABTS was shown to be converted rapidly and, consequently, resulted in faster conversion of the used phenolic lignin model compound (Chapter 4, Scheme $5 C, R=H$ ). Where laccase itself (or in the presence of HOBt) converted the model compound mainly through oligomerisation, ABTS rapidly coupled to the phenolic sub-unit of the model compound and thereby minimised subsequent oligomerisation. Interestingly, coupling of ABTS could not be observed in their earlier work using pyrolysis, ${ }^{[37 b]}$ which clearly indicates how challenging the study of laccase-mediated grafting can be.

In the outlook on future work in Chapter 4, we briefly referred to (ambient) ablation/excitation coupled to mass spectrometric detection to be a potential tool for studying laccase-mediated grafting; it is therefore exciting to see that such tools have now also been employed to do so. Goacher et al. have used Time-of-Flight-Secondary Ion Mass Spectrometry (ToF-SIMS) to study laccase-action on powdered wood. ${ }^{[40]}$ Using their strategy, MS analysis could be performed on the modified powders without requiring additional sample preparation prior to analysis. In this way, it was deemed likely that grafting occurred as the fraction of peaks corresponding to lignin and lignin-like units increased after laccase treatment in the presence of sinapic acid (a lignin sub-unit). In regard to expanding the toolbox of techniques available for probing laccase-mediated grafting, it is worth mentioning that 
Goacher and co-workers have also reviewed multiple techniques for directly monitoring enzymatic treatments on solid material. ${ }^{[41]}$

Richter et al. were able to functionalise lignin through its laccase-mediated coupling with 4aminosulfonic acid (4-ASA) or 4-aminobenzoic acid (4-ABA). ${ }^{[42]}$ By doing so, lignin became soluble at neutral $\mathrm{pH}$ and could therefore be used as concrete dispersing agent. In an exhaustive attempt at finding the right solubilising agent, almost 40 different phenols, anilines and amino acids were reacted in the presence of laccase and lignin. It is interesting that merely the poorly reactive 4-ASA and 4-ABA resulted in laccase solubilisation, where other modifiers of similar (2-ASA), higher (aminohydroxybenzoic acids) or lower (4-aminobenzene-1,3-disulfonic acid) reactivity did not. This is especially surprising, as oligomerisation of 4-ASA or 4-ABA was not observed in control experiments in which lignin was not present, while grafting resulted in significant colouration when lignin was present in the reaction mixture (oligomerised oxidation products of anilines are known to be intensely coloured). It was stated that solid-state NMR on lignin grafted with ${ }^{15} \mathrm{~N}$-labelled 4 -ASA confirmed covalent binding. According to the author of this thesis, this could not be unambiguously concluded in this way. Nonetheless, (isotope-enriched) solid-state NMR might prove to be a valuable tool for studying laccase-mediated grafting in the future.

Building on the work by Schubert and co-workers regarding laccase-mediated iodination of spruce wood, ${ }^{[43]}$ Prosper et al. used this methodology to iodinate bamboo. ${ }^{[44]}$ Similar to the modified spruce wood, modified bamboo showed increased resistance against several fungal strains. This was apparent from a significant increase in the time required to fully mould an iodinated sample (approx. 90 days compared to 40 days for the non-modified samples). As was suggested by us in Chapter 4 , the authors probed whether C-I bond formation could be shown directly using IR spectroscopy. This proved not to be the case as, after modification, no increase in signal intensity was observed at $\bar{v}=500 \mathrm{~cm}^{-1}$. Using inductively coupled plasma mass spectrometry, the amount of iodine was determined to be only minimal (5.41 $\mathrm{mg} \mathrm{g}^{-1}$ bamboo), which is likely the reason that C-I bond formation could not be shown using IR spectroscopy. Using XPS, however, the authors proved that C-I bond formation took place as XPS is very sensitive towards iodine. Apart from peaks corresponding to adsorbed/absorbed iodide (618.8 eV and 630.2 eV) two peaks corresponding to a C-I bond were observed (approx. $621.0 \mathrm{eV}$ and $631.5 \mathrm{eV}$ ). By separating the constituents of bamboo into lignin, hemicellulose and cellulose, they were furthermore able to show that only lignin and hemicellulose (bamboo is rich in ferulic acid-derivatised hemicellulose) ${ }^{[45]}$ were iodinated, which is as expected, as cellulose does not contain phenolic moieties. Interestingly, adsorbed/absorbed iodide contributed approximately equally to the total amount of iodide/iodine for both lignin and hemicellulose even though hemicellulose is known to absorb iodine strongly (similar to starch). ${ }^{[46]}$

The development of new 3D-printable (composite) materials is another hot topic to which laccasemediated surface functionalisation can contribute. Filgueira et al. increased hydrophobicity of thermomechanical wood pulp (TMP) by functionalising it with alkyl gallates in order to increase their adhesion to poly(lactic acid) fibres. ${ }^{[47]}$ The authors cleaned the modified fibres by merely washing them with water, which is, as was stated in Chapter 4, not sufficient to wash away all adsorbed alkyl gallate oligomers, but grafting is nonetheless likely to contribute to functionalisation. Interestingly, modifying TMP with octyl gallate resulted in stronger composites, where modifying with dodecyl gallate decreased composite strength compared to poly(lactic acid) itself. Additionally, 3D printing with the composites including octyl gallate-modified fibres resulted in smoother prints. The use of (modified) TMP/polyethylene composites as 3D printing material appeared not to be beneficial compared to the use of polyethylene itself. ${ }^{[48]}$

Humeau-Virot and co-workers attempted to covalently bind ferulic acid oligomers to pectin (a polysaccharide rich in galacturonic acid monomers). ${ }^{[49]}$ Covalent binding was claimed to have occurred based on the occurrence of 'a new peak' (LC-MS analysis) after reacting together laccase, ferulic acid and a pectin model compound compared to the reaction between ferulic acid and laccase only. Apart from the molecule corresponding to this peak being likely to be build-up from a ferulic acid dimer 
(based on MS-MS analysis), no structural basis for the inclusion of a pectin model compound-based sub-unit had been provided. Furthermore, the model compound used was galacturonic acid, which is a reducing sugar that, in solution, is in equilibrium between its open chain form and furanose/pyranose forms, where pectin mainly consists of $\alpha$-(1-4)-linked monosaccharides in pyranose form. Additionally, the used pectin consisted for $25 \%$ of other sugars; both factors make galacturonic acid resemble pectin only marginally. The use of methyl $\beta$-D-galactopyranosiduronic acid or commercially available methyl $\beta$-D-glucopyranosiduronic acid in combination with other (methylated) monosaccharides present in pectin would resemble it to a far greater extent. This example highlights, once more, the importance of appropriate analytical tools and control experiments.

In an intriguing experiment, Bai and co-workers used laccase to polymerise caffeic acid in the presence of poly(ethylene glycol) (PEG). ${ }^{[50]}$ These polymers proved to be coloured more intensely than polymers prepared in the absence of PEG. This was stated to be caused by an increased number of monomers polymerising through $\mathrm{C}-\mathrm{C}$ bond formation (increasing the length of the $\pi$ system) which, in turn, was attributed to PEG acting as a template to enhance 'linear' polymerisation (Scheme 11). Wool modified by in-situ laccase-mediated polymerisation of caffeic acid had also been coloured more intensely when PEG was present as template. These modified wool samples proved to have a lower electrical resistance while lowering the SWCA of the material to a greater extent than polymerisation without PEG did. In the opinion of the author of this thesis, the latter could be caused by simple inclusion of hydrophilic PEG, but might also partially have been caused by an increased availability of hydroxy moieties due to a lower number of them being converted during 'linear' polymerisation.

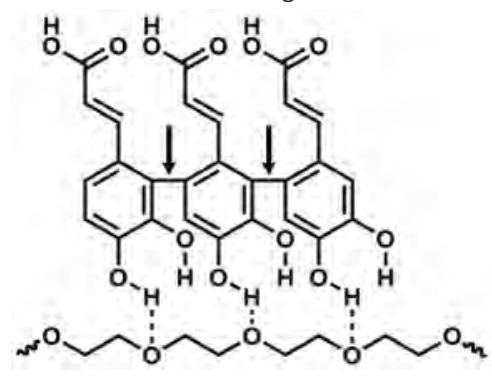

Scheme 11. PEG-templated formation of 'linear' caffeic acid polymers as proposed by Bai and co-workers. ${ }^{[50]}$ The arrows indicate the newly formed bonds between caffeic acid monomers.

In an attempt at using bacterial cellulose as material for cell growth, Zhou et al. covered it with a layer of silk fibroin proteins. ${ }^{[51]}$ Laccase-mediated coupling of model compounds of both materials in the presence of TEMPO showed that covalent bond formation is likely. However, silk fibroin covered the bacterial cellulose approximately equally well with or without laccase, indicating a substantial degree of adsorption in the prior case too. Nonetheless, grafted silk fibroin increased cell viability by $20 \%$ compared to bare bacterial cellulose ( $10 \%$ increase compared to the composite obtained in the absence of laccase).

Stenken and Phillips have used laccase-mediated surface functionalisation with 4-HBA, as was developed in our group, to modify PES membranes to be able to bind heparin through carbodiimidemediated amide-bond formation. ${ }^{[52]}$ By doing so, they were able to alter membrane extraction efficiency for several analytes, although this proved to be highly dependent on the choice of analyte. Nonetheless, as has been documented in Chapter 3, laccase-mediated grafting of 4-HBA does not result in covalent bonding between the membrane and the formed oligomers. Thus, even though this layer is relatively stable in aqueous solutions, coating in this way is not a durable solution for modifying material that is constantly subjected to flow (membranes, microfluidics etc.).

Laccase is continuously being shown to be a versatile enzyme, but also this enzyme has its limits. Chapter 4 and this chapter have summarised a few examples in which laccase was used to covalently modify material in a way that, according to the author of this thesis, has thus far not been shown to 
be mechanistically viable. Examples of this include the binding of keratin or poly(3-hydroxybutyrate) to ethyl cellulose in the absence of a mediator and the coupling of pectin to oligomerised phenolics. When such unlikely covalent bond formation is suggested, it is of even higher importance to study the chemistry behind it.

\section{Outlook}

Laccase-mediated modification of lignocelluloses has in some cases also led to a discussion regarding the underlying functionalisation mechanism. It was suggested that laccase-mediated functionalisation of lignocelluloses using alkyl gallates did not lead to grafting on lignin, but rather to grafting on an unwanted xylan chromophore: hexenuronic acid (Chapter 4, Scheme 15). In our opinion, covalent binding to hexenuronic acid is less likely than binding to lignin as a phenolic radical would be stabilised far more extensively. The use of resin-bound model compounds (hexenuronic acid mimics), as developed in Chapter 3, could aid in clarifying whether it can be covalently functionalised using laccase.

Another showcase of laccase's diversity is its use as ATRP catalyst in polymerisation reactions. In these reactions, obsolete in oxygen, laccase acts as activator by homolytically cleaving the carbonhalide bond of an initiator on the surface or in solution. However, the enzyme mainly acts as a very large host, which does not seem to have any particular advantage over small-molecule catalysts apart from minimising metal contamination of the product in particular cases. ${ }^{[28 b]}$ In order to maintain the latter functionality while minimising non-participating bulk, it is worthwhile to mimic the "ATRPase" active site of laccase. Some "ATRPase" mimics have been developed, but those are mainly based on enzymes having peroxidase activity. ${ }^{[53]}$ These catalysts commonly bear an iron containing hemin or haem group in their active site, which is relatively easy to mimic. However, "ATRPase" iron species can be present as multiple oxidised and active states, complicating their activation mechanism and ironcatalysed ATRP is known to be very sensitive to temperature. ${ }^{[54]}$ Laccase-mimicking ATRP catalysts bearing a strongly bound copper ion would thus be of interest. In order to develop these mimics, one should first determine which copper ion (laccase bears four) is the active species, as current copper containing metalloenzyme mimics used in ATRP reactions do not bear laccase-like copper ions. ${ }^{[55]}$ It was suggested that the copper atom at the $\mathrm{T} 1$ site is responsible for carbon-halide bond cleavage, ${ }^{[28 c]}$ which seems likely as this is the one that is most accessible to organic substrates. The T1 copper site has been the subject of countless research ventures as its varying oxidation potential has intrigued many. Two main motives of this site have been discovered, which both bear a cysteine and two histidine ligands while differing in whether or not a fourth ligand - either a coordinating methionine or a non-coordinating phenylalanine residue - participates in coordination. ${ }^{[56]}$ In both cases the copper ion lies in the plane of the first three binding ligands, a proper mimic would thus resemble this feature while bearing a fourth (non-)coordinating ligand. Some $\mathrm{N}_{2} \mathrm{~S}$ ligands have been developed, ${ }^{[57]}$ but to the best of our knowledge have not yet been employed in copper-catalysed ATRP reactions. Mimicking laccase's "ATRPase" activity is thus still an underexplored topic with vast potential.

\section{Chapter 5. One-step generation of reactive superhydrophobic surfaces via $\mathrm{SiHCl}_{3}$-based silicone nanofilaments}

As laccase-mediated surface modification did not prove to be effective for covalently functionalising poly(ethersulfone), we aimed at modifying synthetic polymers by mildly activating them using chemical agents. Through a two-step strategy involving $\mathrm{Cu}(\mathrm{OAC})_{2}$-catalysed oxidation with $\mathrm{H}_{2} \mathrm{O}_{2}$ as oxidant, followed by a reduction step $\left(\mathrm{NaBH}_{4}\right)$, poly(methyl methacrylate) could be hydroxylated. Subsequent silanisation with $\mathrm{SiHCl}_{3}$ resulted in the formation of an intricate polysiloxane network consisting of so-called silicone nanofilaments. Even though the polysiloxane layer did not possess any hydrophobic moiety, these modified plastics proved to be superhydrophobic (SWCA $>150^{\circ}$, sliding 
angles $\left\langle 1^{\circ}\right.$ ). Due to the presence of $\mathrm{Si}-\mathrm{H}$ groups, the surface could be further functionalised through hydrosilylation.

\section{Remarks}

Although silicone nanofilaments have been proven highly environmentally (weathering) and chemically stable, ${ }^{[58]}$ their mechanical stability is, like that of many other superhydrophobic coatings, ${ }^{[59]}$ very poor. This, unfortunately, hampers their applicability outside a laboratory context. The fragile nanostructure is essential for superhydrophobicity, so current methodologies for minimising mechanical wear focus on bypassing this issue rather than solving it. Seeger and co-workers have, for example, made use of multiscale roughness through which the essential nano-roughness is locally physically shielded from wear by macroscopic topographical alterations. ${ }^{[60]}$ Despite their limitations, silicone nanofilament-coated surfaces have proven effective in multiple applications. ${ }^{[61]}$

\section{Additional research}

Materials possessing both hydrophobic and oleophobic properties are called amphiphobic or omniphobic, and have gained significant attention over recent years. ${ }^{[62]}$ In an attempt at inducing antiwetting properties against multiple liquids we silanised activated PMMA with a mixture of trichlorosilane and trichloro( $1 \mathrm{H}, 1 \mathrm{H}, 2 \mathrm{H}, 2 \mathrm{H}$-heptadecafluorodecyl)silane (CI3F17). I nitial results proved to be highly promising as the unique one-step preparation of superhydrophobic and oleophobic reactive surfaces was achieved (Figure 1, for conditions see $\mathrm{SI}$ ). At a $\mathrm{SiHCl}_{3}$ : Cl3F17 ratio of 1:3 the modified surfaces proved to exhibit static water contact angles of $>150^{\circ}$ and static olive oil contact angles $>100^{\circ}$. This could unfortunately, however, not always be reproduced. Commonly, duplicate experiments revealed that high hydrophobicity could easily be achieved, but that the oleophobic properties of the modified surface differed heavily among multiple experiments/surfaces.

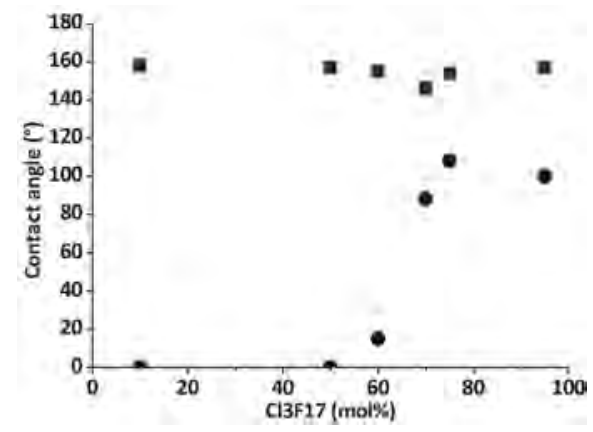

Figure 1. Silanisation of activated PMMA with a mixture of trichlorosilane and trichloro $(1 H, 1 H, 2 H, 2 H$ heptadecafluorodecyl)silane (Cl3F17). Squares: static water contact angle of $9 \mu \mathrm{l}$ droplets and circles: static olive oil contact angle of $4 \mu \mathrm{l}$ droplets on the modified surfaces.

We initially anticipated that poor mixing of extensively fluorinated Cl3F17 and cyclohexane gave rise to this behaviour. Switching to either of the two smaller silanes (trichloro(3,3,3trifluoropropyl)silane (Cl3F3) or trichloro( $1 H, 1 H, 2 H, 2 H$ nonafluorohexyl)silane (Cl3F9)) proved not to increase reproducibility. Similarly, mixing in $1-5 \%$ of a strongly polarised fluoroalkane (methyl nonafluorobutylether or 1 -perfluorooctane) in an attempt at enhancing miscibility did not result in increased reproducibility either. It was alternatively hypothesised that inhomogeneity was the result of local solvation of the PMMA surface. In an attempt at homogeneously solvating the sample surface, activated PMMA was incubated in water-saturated cyclohexane prior to silanisation, unfortunately without success. As expected, oleophobicity correlated strongly with the amount of fluorine on the surface. For those cases where either of the fluoroalkane miscibility agents were applied, successful oleophobisation (static hexadecane contact angle $($ SHDCA $)=92^{\circ}-102^{\circ}$ ) was only achieved for those surfaces where the ratio of polysiloxane-incorporated $\mathrm{Cl} 3 \mathrm{~F} 17$ : $\mathrm{SiHCl}_{3}$ proved high $(0.47-1.4, \mathrm{n}=4$, SI 
Figure S1). Modified surfaces for which the ratio of polysiloxane-incorporated $\mathrm{Cl} 3 \mathrm{~F} 17: \mathrm{SiHCl}_{3}$ was low $(0.012-0.019, \mathrm{n}=3$, SI Figure S2) were fully wetted by hexadecane in all cases. As the ratio of polysiloxane-incorporated $\mathrm{SiHCl}_{3}: \mathrm{Cl} 3 \mathrm{~F} 17$ could not be correlated with the applied conditions, the exact cause underlying these irregularities has thus far not been found.

\section{Outlook}

Although mild oxidation/reduction followed by silanisation proved to be a viable strategy for functionalising both PMMA and cyclic olefin copolymer (COC), ${ }^{[63]}$ the exact mechanism behind the initial activation is unknown. Similar as to the work performed in Chapter 3, studying model compounds might aid in its understanding. As C-H activation of alkanes continues to be an immensely important topic, it is not surprising that Fenton-like copper-mediated oxidation of (cyclic) alkanes has been studied before. Certain copper catalysts proved to selectively hydroxylate the tertiary carbon atoms of 1,2-dimethycyclohexane for example. ${ }^{[64]}$ The same authors have shown that the ratio of products (peroxide/alcohol/carbonyl) resulting from cyclohexane oxidation could differ tremendously, dependent on the used ligand. The catalytic mechanism of these reactions $\left(\mathrm{H}_{2} \mathrm{O}_{2}+\mathrm{Cu}\right.$ or $\left.\mathrm{Fe}\right)$ is furthermore highly dependent on the presence of oxygen as additional oxidant. ${ }^{[65]}$ Although Fentonlike oxidation was originally thought not to proceed through radical species, these studies provided evidence for the participation of (hydroxy) radicals by employing radical scavengers. Furthermore, although less relevant for the current application, the use of additives had been shown to be of major influence on Fenton-like reactions, indicating the complexity of these transformations once more. ${ }^{[66]}$ Oxidation of 1,2-dimethylcyclohexane and that of several polycyclic alkanes ( $m$-chloroperoxybenzoic acid (mCPBA), ${ }^{[67]} \mathrm{O}_{3},{ }^{[68]} \mathrm{RuO}_{4} / \mathrm{NalO}_{4}{ }^{[69]}$ (Scheme 12)) has proven to be selective towards tertiary carbons over secondary carbons (and tertiary bridgehead carbons). The author of this thesis therefore hypothesises that the use of the $\mathrm{Cu}(\mathrm{OAc})_{2} / \mathrm{H}_{2} \mathrm{O}_{2}$ system will mainly result in the oxidation of tertiary carbon atoms in the COC backbone (Scheme 12). Nonetheless, product diversity is expected to be high as the presence of both oxygen and hydrogen peroxide results in the formation of many different radical and charged oxygen species. As none of the reactions mentioned above accurately resembles the $\mathrm{Cu}(\mathrm{OAC})_{2} / \mathrm{H}_{2} \mathrm{O}_{2}$-mediated oxidation of $\mathrm{COC}$, it would be advisable to use a proper model compound like tetrahydrodicyclopentadiene (Scheme 12) and study product formation upon its oxidation with $\mathrm{Cu}(\mathrm{OAc})_{2}$ and $\mathrm{H}_{2} \mathrm{O}_{2}$. This compound is especially valuable as it is has been the subject of multiple theoretical studies examining its combustion/radical formation pathways (tetrahydrodicyclopentadiene is the principal component of high-energy fuel JP-10). ${ }^{[70]}$
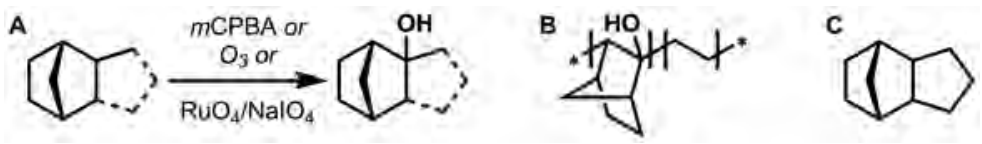

Scheme 12. A: Selective oxidation of a tertiary carbon atom over bridgehead tertiary carbon atoms and secondary carbons. B: Hypothesised main hydroxylation site of $\mathrm{COC}$ resulting from the $\mathrm{Cu}(\mathrm{OAC})_{2} / \mathrm{H}_{2} \mathrm{O}_{2}-\mathrm{NaBH}_{4}$ sequence. C: Plausible COC model compound tetrahydrodicyclopentadiene.

Where these (poly)cyclic alkanes have been studied a lot, especially in the 1980s, product formation from (Fenton-like) oxidation reactions on simple esters has not been studied extensively. In a relatively recent study, Chen and White have oxidised methyl-branched methyl hexanoates and heptanoates by means of peroxidative iron catalysis. ${ }^{[71]}$ Where remote oxidation did occur, oxidation at the $\alpha$ - or $\beta$-position could not be observed. This selectivity was attributed to the electron-withdrawing nature of the ester moiety. As PMMA is merely composed of carbon atoms positioned at the $\alpha$ and $\beta$ sites with respect to the ester moiety, this might therefore be the reason for requiring additional heating to activate PMMA (as compared to the activation of $\mathrm{COC}$ ). Recent studies have shown that simple non-branched esters are commonly not susceptible to activation at their $\beta$ position either; ${ }^{[72]}$ however, methyl cyclopentanecarboxylate could be oxidised at that site. ${ }^{[73]}$ The use of these Fentonlike catalyst systems might alternatively lead to lactonisation and/or hydrolysis. ${ }^{[71,}{ }^{74]}$ In order to 
accurately mimic PMMA activation, studies towards the conversion of PMMA model compounds using our conditions should be performed. PMMA could be mimicked by commercially available methyl 2,2dimethylbutanoate or, more accurately, by methyl 2-ethyl-2-methylbutanoate.

Where silanisation of PMMA using trichlorosilane resulted in the formation of superhydrophobic silicone nanofilaments, this had not been the case for COC. However, as was shown by Seeger and coworkers, superhydrophobisation of several materials could be achieved using methyltrichlorosilane. ${ }^{\text {[75] }}$ It is therefore the opinion of the author of this thesis that silanisation of other materials using $\mathrm{SiHCl}_{3}$ is likely to result in hydrophobisation too. Initial results from silanising other synthetic polymers have confirmed this hypothesis. Acquiring specific wetting by means of silicone nanofilament-growth, however, has proven to, commonly, be highly dependent on water concentration, silane concentration and the ratio between the two. ${ }^{[76]}$ It could therefore be argued that we had been "lucky" with initial successful hydrophobisation of PMMA, however, its superhydrophobisation is remarkably robust. Growth of $\mathrm{SiHCl}_{3}$-based silicone nanofilaments on PMMA proved not to be as sensitive to alterations in the concentration of either reagent or of the silane/water ratio as the growth of most silicone nanofilaments. This behaviour is, however, not unique, as it was also observed for the growth of silicone nanofilaments on poly(ethylene terephthalate) textiles. ${ }^{[61 \mathrm{~d}]}$ Conversely, silicone nanofilamentgrowth on cellulose acetate (which also possesses ester moieties) did not lead to extensive superhydrophobisation (water shedding angle $=55^{\circ}$ ). ${ }^{[60]}$ Successful extensive silanisation is known to benefit from the presence of minute amounts of acid. ${ }^{[76]}$ Usually, in- situ liberation of hydrochloric acid through hydrolysis of the chlorosilane reagent provides the necessary amount of acid. Poly(ethylene terephthalate) is commonly synthesised from benzene-1,4-dicarboxylic acid or through in-situ formation of this compound; [77] incomplete esterification therefore leads to the presence of surfacebound carboxylic acids. Similarly, PMMA is known to be slightly acidic, possibly due to the presence of carboxylic acid moieties. ${ }^{[78]}$ Partial hydrolysis of ester-containing cellulose acetate would not result in surface-bound carboxylic acid moieties. We therefore would argue that, although preliminary, higher surface acidity enhances silanisation efficiency. If true, partial hydrolysis of PMMA's ester moieties as a side effect of our employed copper acetate activation strategy, might provide an additional explanation for why silanisation is more efficient on activated PMMA. This hypothesis could be further tested by evaluating silanisation on copolymers of methacrylic acid and methyl methacrylate in which the ratio between both monomers is varied.

As a proof of principle, hydrosilylation was performed using fluorinated alkenes and alkynes. It should be remarked that this, unfortunately, did not lead to oleophobic surfaces. The use of differently functionalised alkenes or alkynes could provide additional reactivity by, for example, installing an ATRP initiator (allyl 2-bromo-2-methylpropionate). By doing so, (harsh plasma activation and) synthesis of the initiator under stringent water-free conditions in order to allow for immobilisation on a hydroxyfunctionalised polysiloxane would not be necessary. ${ }^{[61 a, 79]}$

Superhydrophobicity arises when water is not able to penetrate the intricate nanostructure of a certain material, which is thus filled with gas. Lei and co-workers made use of this property in order to design a biosensor for enhanced direct glucose detection. Glucose oxidase enzymes adsorbed on a superhydrophobic platinum electrode showed increased hydrogen peroxide production as a result of higher oxygen availability due to better diffusion of oxygen in air/gas (within the nanostructure) compared to that within the aqueous analyte (glucose) solution. ${ }^{\left[{ }^{[0]}\right.}$ Our reactive silicone nanofilamentcoated surface should allow for installing an anchor for protein immobilisation to induce the possibility of covalently binding an enzyme (Scheme 13). Amine anchors have already been shown to allow for covalent immobilisation of glucose oxidase, ${ }^{[81]}$ which could thereby thus pave the way for the use of reactive silicone nanofilament-coated surfaces as biosensor platform. 

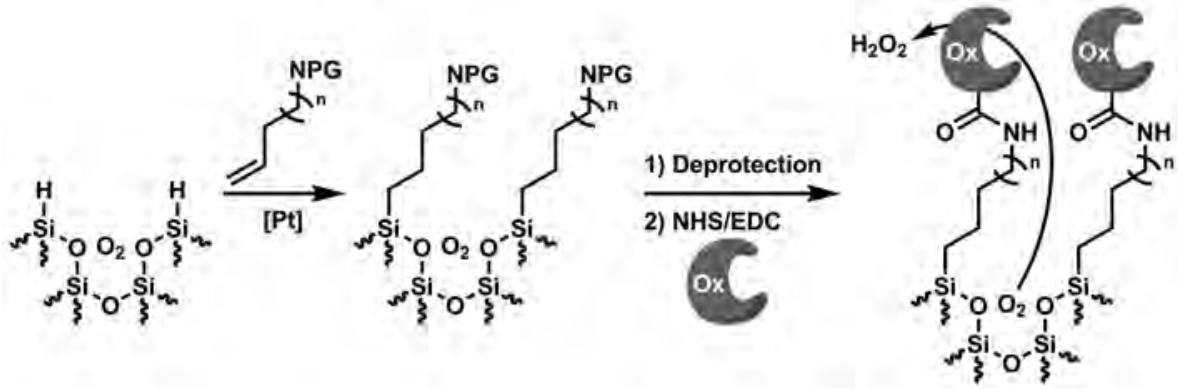

Scheme 13. Platinum-catalysed functionalisation of superhydrophobic $\mathrm{SiHCl}_{3}$-based silicone nanofilaments with a protected amine (NPG) anchor for the immobilisation of oxidase enzymes (Ox) for enhanced hydrogen peroxide production. $\mathrm{NHS}=\mathrm{N}$-hydroxysuccinimide, $\mathrm{EDC}=1$-ethyl-3-(3-dimethylaminopropyl)carbodiimide.

\section{References}

[1] N. Nady, K. Schroën, M. C. R. Franssen, B. van Lagen, S. Murali, R. M. Boom, M. S. Mohyeldin, H. Zuilhof, ACS Appl. Mater. Interfaces 2011, 3, 801-810.

[2] R. Hermann, S. Naumov, G. R. Mahalaxmi, O. Brede, Chem. Phys. Lett. 2000, 324, 265-272.

[3] R. F. Anderson, K. B. Patel, M. R. L. Stratford, J. Chem. Soc., Faraday Trans. 1 1987, 83, 3177-3187.

[4] R. C. Simon, E. Busto, N. Richter, V. Resch, K. N. Houk, W. Kroutil, Nat. Commun. 2016, 7, 13323.

[5] E. N. Pitsinos, V. P. Vidali, E. A. Couladouros, Eur. J. Org. Chem. 2011, 2011, 1207-1222.

[6] T.-S. Wu, Y.-L. Tsai, A. G. Damu, P.-C. Kuo, P.-L. Wu, J. Nat. Prod. 2002, 65, 1522-1525.

[7] N. Jung, S. Bräse, Eur. J. Org. Chem. 2009, 2009, 4494-4502.

[8] F. Theil, Angew. Chem. Int. Ed. 1999, 38, 2345-2347.

[9] L.-S. Shi, P.-C. Kuo, Y.-L. Tsai, A. G. Damu, T.-S. Wu, Bioorg. Med. Chem. 2004, 12, 439-446.

[10] T.-S. Wu, C.-C. Hwang, P.-C. Kuo, T.-H. Kuo, A. G. Damu, C.-R. Su, Chem. Pharm. Bull. 2004, 52, 1227-1230.

[11] N. Otto, D. Ferenc, T. Opatz, J. Org. Chem. 2017, 82, 1205-1217.

[12] A. M. Mfuh, J. D. Doyle, B. Chhetri, H. D. Arman, O. V. Larionov, J. Am. Chem. Soc. 2016, 138, 2985-2988.

[13] N. Nady, A. H. El-Shazly, Desalin. Water Treat. 2015, 55, 2996-3002.

[14] J. Ihssen, M. Schubert, L. Thöny-Meyer, M. Richter, PLoS One 2014, 9, e89924.

[15] E. Enaud, M. Trovaslet, F. Naveau, A. Decristoforo, S. Bizet, S. Vanhulle, C. Jolivalt, Enzyme Microb. Technol. 2011, 49, 517-525.

[16] R. I keda, H. Uyama, S. Kobayashi, Macromolecules 1996, 29, 3053-3054.

[17] M. Khayet, J. P. G. Villaluenga, M. P. Godino, J. I. Mengual, B. Seoane, K. C. Khulbe, T. Matsuura, J. Colloid Interface Sci. 2004, 278, 410-422.

[18] T. Xu, D. Wu, L. Wu, Prog. Polym. Sci. 2008, 33, 894-915.

[19] H.-S. Dang, E. A. Weiber, P. J annasch, J. Mater. Chem. A 2015, 3, 5280-5284.

[20] Y. Xue, H. Xiao, Y. Zhang, Int. J. Mol. Sci. 2015, 16, 3626-3655.

[21] a) M. Schroeder, N. Aichernig, G. M. Guebitz, V. Kokol, Biotechnol. J. 2007, 2, 334-341; b) P. Widsten, C. Heathcote, A. Kandelbauer, G. M. Guebitz, G. S. Nyanhongo, E. Nugroho Prasetyo, T. Kudanga, Process Biochem. 2010, 45, 1072-1081.

[22] a) T. Maximilian, P. Gabriele, O. Knut, H. Ulrike, ChemMedChem 2012, 7, 22-31; b) M. C. Jennings, K. P. C. Minbiole, W. M. Wuest, ACS Infect. Dis. 2015, 1, 288-303.

[23] Z. Jia, D. Shen, W. Xu, Carbohydr. Res. 2001, 333, 1-6. 
[24] a) A. B. Albadarin, M. N. Collins, M. Naushad, S. Shirazian, G. Walker, C. Mangwandi, Chem. Eng. J. 2017, 307, 264-272; b) V. Nair, A. Panigrahy, R. Vinu, Chem. Eng. J. 2014, 254, $491-$ 502.

[25] F. d'Acunzo, C. Galli, P. Gentili, F. Sergi, New J. Chem. 2006, 30, 583-591.

[26] A. M. Socha, R. Parthasarathi, J. Shi, S. Pattathil, D. Whyte, M. Bergeron, A. George, K. Tran, V. Stavila, S. Venkatachalam, M. G. Hahn, B. A. Simmons, S. Singh, Proc. Natl. Acad. Sci. 2014, 111, E3587-E3595.

[27] A. M. Socha, S. Singh, B. A. Simmons, M. Bergeron (University of California, National Technology and Engineering Solutions of Sandia LLC), US9765044B2, 2017.

[28] a) Y.-H. Ng, F. di Lena, C. L. L. Chai, Chem. Commun. 2011, 47, 6464-6466; b) C. Fodor, B. Gajewska, O. Rifaie-Graham, E. A. Apebende, J. Pollard, N. Bruns, Polym. Chem. 2016, 7, 6617-6625; c) Y.-H. Ng, F. di Lena, C. L. L. Chai, Polym. Chem. 2011, 2, 589-594.

[29] G. Gao, M. A. Karaaslan, J. F. Kadla, F. Ko, Green Chem. 2014, 16, 3890-3898.

[30] a) J. B. Schlenoff, Langmuir 2014, 30, 9625-9636; b) M. Hadidi, A. L. Zydney, J. Membr. Sci. 2014, 452, 97-103; c) T. Cai, X. Li, C. Wan, T.-S. Chung, J. Membr. Sci. 2016, 497, 142-152.

[31] Z. Cao, S. Jiang, Nano Today 2012, 7, 404-413.

[32] J. Kuang, P. B. Messersmith, Langmuir 2012, 28, 7258-7266.

[33] C. Díaz Blanco, A. Ortner, R. Dimitrov, A. Navarro, E. Mendoza, T. Tzanov, ACS Appl. Mater. Interfaces 2014, 6, 11385-11393.

[34] F. Hollmann, Y. Gumulya, C. Tölle, A. Liese, O. Thum, Macromolecules 2008, 41, 8520-8524.

[35] A. B. Sieval, A. L. Demirel, J. W. M. Nissink, M. R. Linford, J. H. van der Maas, W. H. de J eu, H. Zuilhof, E. J. R. Sudhölter, Langmuir 1998, 14, 1759-1768.

[36] M. P. Stewart, J. M. Buriak, Angew. Chem. Int. Ed. 1998, 37, 3257-3260.

[37] a) T. Kudanga, E. Nugroho Prasetyo, J. Sipilä, G. S. Nyanhongo, G. M. Guebitz, Enzyme Microb. Technol. 2010, 46, 272-280; b) L. Munk, A. M. Punt, M. A. Kabel, A. S. Meyer, RSC Adv. 2017, 7, 3358-3368.

[38] a) S. K. Hanson, R. Wu, L. A. P. Silks, Angew. Chem. Int. Ed. 2012, 51, 3410-3413; b) B. Sedai, C. Díaz-Urrutia, R. T. Baker, R. Wu, L. A. P. Silks, S. K. Hanson, ACS Catal. 2013, 3, 3111-3122.

[39] R. Hilgers, J.-P. Vincken, H. Gruppen, M. A. Kabel, ACS Sustainable Chem. Eng. 2018, 6, 2037-2046.

[40] R. E. Goacher, E. J. Braham, C. L. Michienzi, R. M. Flick, A. F. Yakunin, E. R. Master, Physiol. Plant. DOI: https://doi.org/10.1111/ppl.12688.

[41] R. E. Goacher, M. J. Selig, E. R. Master, Curr. Opin. Biotechnol. 2014, 27, 123-133.

[42] J. Dagmara, H. Tobias, S. Mark, Y. Alpaslan, W. Christophe, R. Daniel, S. Irene, R. Michael, ChemBioChem 2018, 19, 1365-1369.

[43] M. Schubert, J. Engel, L. Thöny-Meyer, F. W. M. R. Schwarze, J. Ihssen, Appl. Environ. Microbiol. 2012, 78, 7267-7275.

[44] N. K. Prosper, S. Zhang, H. Wu, S. Yang, S. Li, F. Sun, B. Goodell, Wood Sci. Technol. 2018, 52, 619-635.

[45] D. M. de Oliveira, A. Finger-Teixeira, T. Rodrigues Mota, V. H. Salvador, F. C. Moreira-Vilar, H. B. Correa Molinari, R. A. Craig Mitchell, R. Marchiosi, O. Ferrarese-Filho, W. Dantas dos Santos, Plant Biotechnol. J. 2015, 13, 1224-1232.

[46] M. H. O'Dwyer, Biochem. J. 1939, 33, 713-717.

[47] D. Filgueira, S. Holmen, J. K. Melbø, D. Moldes, A. T. Echtermeyer, G. Chinga-Carrasco, ACS Sustainable Chem. Eng. 2017, 5, 9338-9346.

[48] D. Filgueira, S. Holmen, J. K. Melbø, D. Moldes, A. T. Echtermeyer, G. Chinga-Carrasco, Polymers 2018, 10, 314.

[49] N. Karaki, A. Aljawish, L. Muniglia, S. Bouguet-Bonnet, S. Leclerc, C. Paris, J. Jasniewski, C. Humeau-Virot, Enzyme Microb. Technol. 2017, 104, 1-8. 
[50] R. Bai, Y. Yu, Q. Wang, X. Fan, P. Wang, J. Yuan, J. Shen, J. Cleaner Prod. 2018, 191, 48-56.

[51] Q. Zhou, Q. Zhang, P. Wang, C. Deng, Q. Wang, X. Fan, Fibers Polym. 2017, 18, 1478-1485.

[52] S. J. Phillips, J. A. Stenken, Anal. Chem. 2018, 90, 4955-4960.

[53] a) H. Zhou, W. Jiang, N. An, Q. Zhang, S. Xiang, L. Wang, J. Tang, RSC Adv. 2015, 5, 4272842735; b) W. Jiang, Y. Pan, J. Yang, Y. Liu, Y. Yang, J. Tang, Q. Li, J. Colloid Interface Sci. 2018, 521, 62-68; c) K. Yamashita, K. Yamamoto, J.-i. Kadokawa, Polymer 2013, 54, 17751778.

[54] H. Schroeder, D. Yalalov, M. Buback, K. Matyjaszewski, Macromol. Chem. Phys. 2012, 213, 2019-2026.

[55] A. Sanchez-Sanchez, A. Arbe, J. Kohlbrecher, J. Colmenero, J. A. Pomposo, Macromol. Rapid Commun. 2015, 36, 1592-1597.

[56] S. M. Jones, E. I. Solomon, Cell. Mol. Life Sci. 2015, 72, 869-883.

[57] a) L. Zhou, D. Powell, K. M. Nicholas, Inorg. Chem. 2007, 46, 7789-7799; b) K. C. Tran, J. P. Battioni, J. L. Zimmermann, C. Bois, G. J. A. A. Koolhaas, P. Leduc, E. Mulliez, H. Boumchita, J. Reedijk, J. C. Chottard, Inorg. Chem. 1994, 33, 2808-2814; c) T. Tano, K. Mieda, H. Sugimoto, T. Ogura, S. Itoh, Dalton Trans. 2014, 43, 4871-4877.

[58] a) J. Zimmermann, F. A. Reifler, U. Schrade, G. R. J. Artus, S. Seeger, Colloids Surf. A 2007 302, 234-240; b) J. Zimmermann, G. R. J. Artus, S. Seeger, Appl. Surf. Sci. 2007, 253, 59725979.

[59] B. P. Dyett, A. H. Wu, R. N. Lamb, ACS Appl. Mater. Interfaces 2014, 6, 18380-18394.

[60] J. Zimmermann, F. A. Reifler, G. Fortunato, L.-C. Gerhardt, S. Seeger, Adv. Funct. Mater. 2008, 18, 3662-3669.

[61] a) Y. I wasaki, Y. Omichi, R. I wata, Langmuir 2008, 24, 8427-8430; b) J. Zimmermann, M. Rabe, D. Verdes, S. Seeger, Langmuir 2008, 24, 1053-1057; c) L. Li, B. Li, L. Wu, X. Zhao, J. Zhang, Chem. Commun. 2014, 50, 7831-7833; d) J. Zhang, S. Seeger, Adv. Funct. Mater. 2011, 21, 4699-4704.

[62] a) M. Liu, Y. Zheng, J. Zhai, L. Jiang, Acc. Chem. Res. 2010, 43, 368-377; b) A. Tuteja, W. Choi, J. M. Mabry, G. H. McKinley, R. E. Cohen, Proc. Natl. Acad. Sci. 2008, 105, 1820018205; c) Z. L. Chu, S. Seeger, Chem. Soc. Rev. 2014, 43, 2784-2798.

[63] R. R. Carvalho, S. P. Pujari, E. X. Vrouwe, H. Zuilhof, ACS Appl. Mater. Interfaces 2017, 9, 16644-16650.

[64] I. Garcia-Bosch, M. A. Siegler, Angew. Chem. 2016, 128, 13065-13068.

[65] P. Stavropoulos, R. Çelenligil-Çetin, A. E. Tapper, Acc. Chem. Res. 2001, 34, 745-752.

[66] G. B. Shul'pin, J. Mol. Catal. A 2002, 189, 39-66.

[67] N. Takaishi, H. Takahashi, O. Yamashita, Y. Inamoto, J. Org. Chem. 1986, 51, 4862-4865.

[68] Z. Cohen, E. Keinan, Y. Mazur, T. H. Varkony, J. Org. Chem. 1975, 40, 2141-2142.

[69] A. Tenaglia, E. Terranova, B. Waegell, J. Chem. Soc., Chem. Commun. 1990, 1344-1345.

[70] a) L. Türker, S. Variş, Ç. Çelik Bayar, Fue/ 2013, 104, 128-132; b) J. M. Hudzik, R. Asatryan, J. W. Bozzelli, J. Phys. Chem. A 2010, 114, 9545-9553.

[71] M. S. Chen, M. C. White, Science 2007, 318, 783-787.

[72] L. Gómez, M. Canta, D. Font, I. Prat, X. Ribas, M. Costas, J. Org. Chem. 2013, 78, 1421-1433.

[73] M. S. Chen, M. C. White, Science 2010, 327, 566-571.

[74] Q. Zhang, C. Wang, Y. Lei, J. Adv. Oxid. Technol. 2016, 19, 125-133.

[75] G. R. J. Artus, S. J ung, J. Zimmermann, H. P. Gautschi, K. Marquardt, S. Seeger, Adv. Mater. 2006, 18, 2758-2762.

[76] J. Zhang, A. Wang, S. Seeger, Polym. Chem. 2014, 5, 1132-1139.

[77] H. Köpnick, M. Schmidt, W. Brügging, J. Rüter, W. Kaminsky in Ullmann's Encyclopedia of Industrial Chemistry, Wiley-VCH Verlag, Weinheim, 2000.

[78] I. A. Starostina, N. V. Makhrova, O. V. Stoyanov, I. V. Aristov, J. Adhes. 2012, 88, 751-765. 
[79] a) G. Carrot, S. Diamanti, M. Manuszak, B. Charleux, J. P. Vairon, J. Polym. Sci. A Polym. Chem. 2001, 39, 4294-4301; b) S. Munirasu, R. G. Karunakaran, J. Rühe, R. Dhamodharan, Langmuir 2011, 27, 13284-13292; c) J. Li, B. Hu, K. Yang, B. Zhao, J. S. Moore, ACS Macro Lett. 2016, 5, 819-822.

[80] L. Yongjiu, S. Ruize, Z. Xiangcheng, F. Xinjian, J. Lei, Adv. Mater. 2016, 28, 1477-1481.

[81] J. M. Alonso, A. A. M. Bielen, W. Olthuis, S. W. M. Kengen, H. Zuilhof, M. C. R. Franssen, Appl. Surf. Sci. 2016, 383, 283-293. 


\section{Supporting information corresponding to Chapter 6}

Scheme S1. Commercially available (dialkylamino)methyl-substituted phenols (19-25).<smiles>CC(=O)c1ccc(O)c(CN(C)C)c1O</smiles>

19<smiles>CC1=CC(C)(C)Nc2cc(CN(C)C)c(O)cc21</smiles>

ane (20) ene (21)<smiles>Oc1ccc2ccccc2c1CN1CCCCC1</smiles>

Dimethylamine (22) Piperidine (23)<smiles></smiles>

$R^{1}=H, R^{2}=M e, Y=C_{2}(24)$

$R^{\prime}=M e, R^{2}=H, Y=O(25)$

Commercially available (dialkylamino)methyl-substituted phenols $\left(<150 € \mathrm{~g}^{-1}\right)$ (19-25) of which quaternisation of their tertiary amine groups would lead to positively charged phenols $\left(\mathbf{1 9}^{+}-\mathbf{2 5}^{+}\right)$as interesting targets for use in laccase-mediated grafting.

\section{Experimental}

\section{Chemicals}

Trichloro( $1 H, 1 H, 2 H, 2 H$ heptadecafluorodecyl)silane ( $>96 \%$ ) and methyl nonafluorobutylether isomers ( $>98 \%$ ) were obtained from TCl Europe. $1 /$-Perfluorooctane had been supplied by Fluorochem. Olive oil was bought at a local retailer and used as is.

\section{PMMA surface activation}

If not mentioned otherwise, activation was conducted under the conditions mentioned in Chapter 5. The first step of activation (oxidation) had alternatively been performed by, during sonication, heating up the mixture to $35^{\circ} \mathrm{C}$ (Act35) rather than to $50^{\circ} \mathrm{C}$ (Act50).

\section{Silanisation of activated PMMA}

Silanisation using a mixture of $\mathrm{SiHCl}_{3}$ and $\mathbf{C l} 3 \mathbf{F 1 7}$. In a glovebox, trichlorosilane $(3.5,14,17.5$, 21,35 or $65 \mathrm{mM}$ final concentration) and trichloro( $1 H, 1 H, 2 H, 2 H$-heptadecafluorodecyl)silane (Cl3F17, $66.5,56,52.5,49,35$ or $5 \mathrm{mM}$ final concentration respectively) were mixed in anhydrous cyclohexane ( 5.0 or $1.3 \mathrm{ml}$ ). One or two activated PMMA (Act35) samples were placed in the solution, after which they were allowed to stand for $20 \mathrm{~min}$ at room temperature. Rinsing with cyclohexane and drying under a stream of nitrogen afforded superhydrophobic silicone nanofilament-coated PMMA with varying oleophobicity (Chapter 6, Figure 1 ). These experiments were also repeated with activated PMMA (Act50) at lower silane concentrations, using water-saturated cyclohexane and combinations thereof, which conditions were thereby similar to those employed in Chapter 5.

Silanisation using a mixture of $\mathrm{SiHCl}_{3}, \mathrm{Cl} 3 \mathrm{~F} 17$ and a miscibility agent. ${ }^{[1]}$ In a glovebox, trichloro( $1 H, 1 H, 2 H, 2 H$-heptadecafluorodecyl)silane (Cl3F17, $0.40 \mathrm{mM}$ final concentration) was dissolved in either methyl nonafluorobutylether or $1 /$-perfluorooctane (150, 300 or $750 \mu l)$. Addition of water-saturated cyclohexane $(14.85,14.70$ or $14.25 \mathrm{ml}$ respectively, $3.5 \mathrm{mM} \mathrm{H} 2 \mathrm{O}$ ) was followed by addition of trichlorosilane (1.2 mM final concentration) and the mixture was thoroughly shaken. One or two activated PMMA (Act50) samples were placed (marking downwards) in the solution, after which the vials were tightly closed and taken out of the glovebox. After being wrapped with Parafilm ${ }^{\circledR}$, the mixture was sonicated for $2 \mathrm{~min}$ and subsequently shaken at 80-110 rpm for 1, 2, 3, 4 or 6 h. Cleaning 
of the samples was thereafter achieved by placing the samples (marking downwards) in fresh cyclohexane and subsequent shaking, after which the cyclohexane was replenished and the vial was shaken again (repeated twice). Rinsing with cyclohexane and drying under a stream of nitrogen afforded superhydrophobic (and oleophobic) silicone nanofilament-coated PMMA.

\section{Analysis}

Static hexadecane/ olive oil contact angle measurements. Static hexadecane/olive oil contact angle measurements were conducted similarly to the SWCA measurements reported in Chapter 5, but with $4 \mu \mathrm{l}$ droplets of either of the two oils instead of water.

X-ray photoelectron spectroscopy (XPS). XPS analysis has been conducted according to the procedure outlined in Chapter 5, but spectra were calibrated according to the $\mathrm{CF}_{3}$ signal $(394.0 \mathrm{eV}){ }^{[2]}$ Silanisation with a mixture of $\mathrm{SiHCl}_{3}$ and $\mathrm{Cl} 3 \mathrm{~F} 17$ introduced two new elements (silicon and fluorine) of which fluorine could only be introduced through incorporation of Cl3F17 in the polysiloxane layer. This allowed us to estimate to what extent either of the reagents had participated in surface-bound polysiloxane formation. \%F 1s / $17=$ "Cl3F17" and \%Si 2p - "Cl3F17" = "SiHCl3", thus an estimate of the ratio between the two surface-bound species could be calculated through " $\mathrm{Cl} 3 \mathrm{~F} 17$ " / "SiHCl 3 ". Each value mentioned in Chapter 6 represents an average over measurements on two or four spots on a single surface.

Figure S1. XPS wide scan of an oleophobic surface generated through silanisation with a mixture of $\mathrm{SiHCl}_{3}, \mathrm{Cl} 3 \mathrm{F17}$ and a miscibility agent.

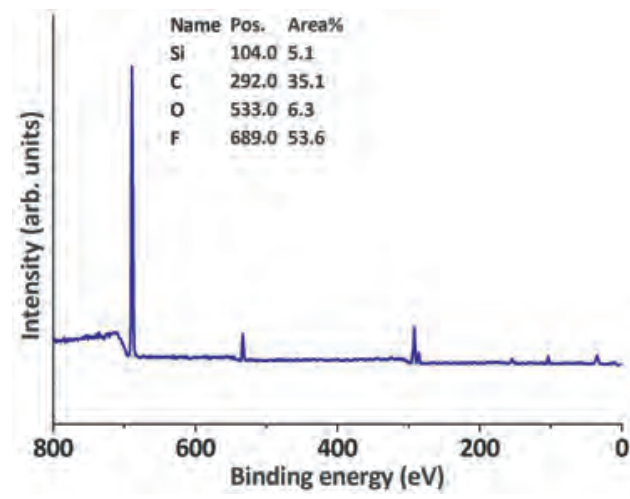

Cl3F17: $\mathrm{SiHCl}_{3}=1.6$.

SHDCA of corresponding surface $=102^{\circ}$.
Figure S2. XPS wide scan of a nonoleophobic surface generated through silanisation with a mixture of $\mathrm{SiHCl}_{3}, \mathrm{Cl}_{3} \mathrm{~F} 17$ and a miscibility agent.

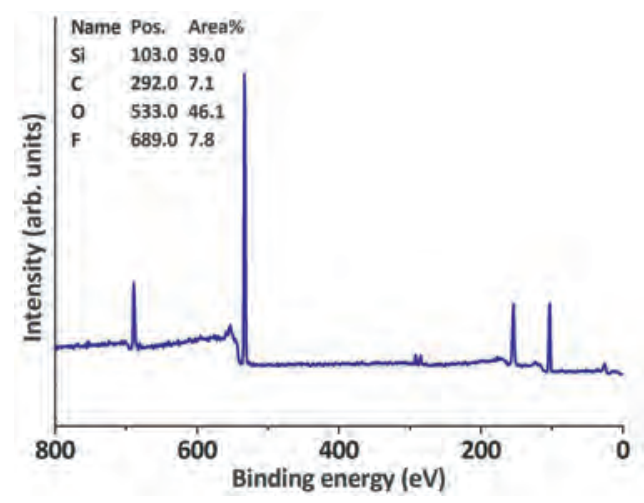

$\mathrm{Cl} 3 \mathrm{~F} 17: \mathrm{SiHCl}_{3}=0.03$.

Surface was fully wetted by hexadecane.

\section{References}

[1] J. Zhang, A. Wang, S. Seeger, Polym. Chem. 2014, 5, 1132-1139.

[2] K. Ellinas, S. P. Pujari, D. A. Dragatogiannis, C. A. Charitidis, A. Tserepi, H. Zuilhof, E. Gogolides, ACS Appl. Mater. Interfaces 2014, 6, 6510-6524. 


\section{Summary}

Chapter 1 provides the required background knowledge for any chemically oriented scholar to comprehend the interdisciplinary work presented herein. Crucial topics, such as polymer surface modification and analysis, wetting behaviour and adhesion prevention were introduced. Current methodologies for modifying polymer surfaces typically require harsh chemicals and conditions. The research described herein has therefore been focussed on acquiring a better understanding and increasing the scope of novel tools for mildly modifying polymers.

One of these novel tools is the laccase-mediated surface functionalisation of poly(ethersulfone) membranes using 4-hydroxybenzoic acid (4-HBA). The resulting overlayer minimised membrane fouling by several biofoulants. In order to comprehend the underlying functionalisation mechanism, the solution-phase oligomerisation of 4-HBA had to be studied first, which is described in Chapter 2. Initial conversion of 4-HBA proved to occur only slowly and resulted in two main products: a C3-C3'bound and a C3-O-bound dimer. A plurality of other products were found after $24 \mathrm{~h}$ of incubation, which included a C1-C3'-bound and possibly a C1-O-bound dimer. Furthermore, laccase-mediated conversion of these dimers proved to be far more rapid than conversion of 4-HBA itself, and correlated strongly with the abundance of the individual dimers. The influence of dimer reactivity on their abundance was confirmed by quantum chemical calculations. These findings provided us with handles for designing phenols with enhanced reactivity and controlled binding profiles.

We used the gained knowledge to synthesise novel positively charged phenolic monomers that were anticipated to, upon laccase-mediated surface functionalisation, introduce anti-bacterial properties to the membrane while allowing it to be used as support membrane for layer-by-layer deposition. As is described in Chapter 3, however, in-situ laccase-mediated conversion of these phenolics did not lead to significant surface functionalisation. In order to understand why functionalisation was achieved for other monomers (i.e. 4-HBA), 4-HBA, laccase and any of several PES model compounds were incubated together and the resultant mixture was studied using LC-MS. However, no covalent bond formation between (oligomeric) 4-HBA and either of the soluble, insoluble or resin-bound PES model compounds could be observed. The use of phenols bearing negatively charged substituents did also not lead to membrane surface modification. Finally, membranes having an overlayer of oligomeric 4-HBA proved to be extensively decolourised upon washing with a detergent solution. Considering all of the above, it was concluded that laccase-mediated surface modification resulted from strong physisorption, rather than from covalent grafting of oligomeric 4-HBA.

As it was challenging to reveal the mechanisms underlying our functionalisation strategy, we anticipated that other researchers might also have encountered similar challenges. It is therefore that in Chapter 4 recently published laccase-mediated surface modification strategies are discussed and assessed on whether grafting is likely to have occurred. This assessment was based on five factors: mechanistic rationale, pre-treatment, control experiments, washing/cleaning and the used analytical tools. Generally speaking, laccase-mediated grafting on lignocelluloses proved to be likely. Quite commonly, however, grafting coincided with physical adsorption due to insufficient washing. We concluded that a lack of proper surface analyses and studies towards the mechanisms underlying grafting on polysaccharides, proteins and synthetic polymers regularly hampered achieving covalent grafting on these materials.

Apart from enzymatic surface modification, additional chemical strategies for achieving mild polymer functionalisation were assessed too. PMMA activation was accordingly achieved through peroxidative copper catalysis, followed by sodium borohydride reduction to result in surface hydroxylation. As was described in Chapter 5, this offered a platform for the robust growth of $\mathrm{SiHCl}_{3-}$ based silicone nanofilaments, while maintaining polymer transparency. Due to their intricate nanostructure, these silicone nanofilaments granted superhydrophobicity (SWCA $>150^{\circ}$, sliding angles $\left\langle 1^{\circ}\right.$ ) to the material. The presence of $\mathrm{Si}-\mathrm{H}$ moieties on the surface allowed for further 
functionalisation through hydrosilylation. As a proof of principle, we employed platinum-catalysed hydrosilylation to decorate the surface with extensively fluorinated alkenes and alkanes. This fluorinated exterior provided the material with protection towards hydrolytic degradation. We have thereby developed the first intrinsically superhydrophobic reactive silicone nanofilament-coated transparent polymer surface.

Finally, Chapter 6 summarises the highlights of previous chapters, while offering an in-depth discussion on possible improvements and future work. 


\section{Acknowledgements}

$\sim$ Somehow, this part of the thesis is remarkably challenging.

I would like to start by saying that the past four and a half years have allowed me to grow as a human being, giving me the chance to be myself. I want to, especially, thank Bianca for that, but definitely also the ORC team. Most of you have been generating a positive and non-judgemental environment, which I have not experienced as such before starting in Wageningen.

Maurice, I want to thank you for bearing with me for four years; we had a rough and unprofitable start, but eventually, I think, we can both be happy and proud with the result. I especially appreciated the fact that every paper or chapter we produced was important to you, regardless of it being published in Science (one is allowed to hope), RSC Advances or any other journal for that matter. You were, furthermore, always open to my ideas and you gave me the chance to execute them. Finally, your door, literally, always being open has helped me tremendously.

I want to thank you, Han, for being my promotor and thereby for allowing me to start and repower the project. It was your idea to study mild chemical oxidation of synthetic polymers, which, if you had not initiated this, had never led to our discovery of unique superhydrophobic reactive silicone nanofilaments. I specifically admire your talent for spotting research opportunities and exploit those to the fullest.

As for the rest of ORC, I would like to specifically thank my colleagues with whom I shared a serene and peaceful office at the Dreijen: Aline, Tjerk and Jorick. Which upon moving to Helix completely shifted towards utter chaos, positive chaos, but nonetheless chaos, thanks to J orin, Ian and J ordi. I have shared laboratory space with many, but special thanks go out to "labmamma" J udith of whom I once stated that I would not take on any other position which would not include her. I sincerely miss the darts-breaks with, among others, Bas (one-man word joke audience) and Frank. I gladly boosted the ego of the latter by winning exactly one game of table tennis against him $(n=\infty)$. I deeply appreciate Esther and Annemieke for not only wanting to be my paranymphs, but even more so for always offering a sympathetic ear. Many, but far too little, gaming nights have been shared with Sweccha and most of those mentioned above. Sidhu, you are one of the most kind and helpful people I have ever had the privilege to meet. Many thanks go out to J orge and Rui for their help with my first and fourth paper. PepEin, I really miss your NMR input, helpfulness and cheerful presence, and most of all being able to share word jokes. I am very much strengthened by the warmth that you bring to ORC every day, thank you for that Hendra. J elmer, I think I speak for all of us by saying that you were a very positive asset to the group. What will follow now is a list of people of ORC who have all, in some way or another, contributed to my PhD: Ai, Albert, Aleida, Alexandre, Alice, Andrada, Andrii, Anke, Anne-Marie, Barend, Bauke, Bep, Bram, Carel, Caroline, Christie, DJ, Elbert, Elly, Erik, Esther, Fátima, Florine, Floris, Frank, Fred, Ghazal, Gina, Henny, Jacob, Linda, Louis, Maarten, Medea, Meta, Michel, Milou, Nagendra, Nathalja, PepZwei, Rick, Satesh, Sevil, Stefanie, Teris, Tom, Ton, Txema, Umesh, Vincent, Wilco, Willem, Wouter, Yao, Zhanhua. I also want to thank all students that were part of ORC and those that I had the pleasure to supervise during practical courses. You have been an essential part of my time in Wageningen and you remind me of how I will likely never fully outgrow that part of me that still is a student.

When it comes to students, there are three that should be more extensively noted. Meral, I am happy to be able to share my hatred for machines (HPLC pumps in this case) with you and honoured that you decided to become my first student. I also want to thank you, Marian, for being one of the brightest students I have ever met, it is just a shame that laccase did not do what we wanted it to do. I hope I am still in time; I think you would be a good candidate for a PhD in the field of organic chemistry. And last but not least: Wendy! You are a very positive and uplifting person and I am happy that, although you might have been lured to ORC under false pretences ("green" is a relative term (:)), we have a publication together. 
My highly interdisciplinary PhD trajectory was severely strengthened by its external collaborations. In that regard, NanoNextNL has proven to be the main player by both providing essential monetary support and bringing together the project partners from Pentair X-flow, University of Twente, KWR and Wageningen University. I am grateful for the meetings we had and especially for the introduction in practical membrane handling at Pentair X-flow, I would therefore like to thank Arjen Kemperman, Emile Cornelissen, Erik Vriezekolk, Jens Potreck, Karin Schroën, Kitty Nijmeijer, Wiebe de Vos, Jeroen Ploegmakers, Melvin Kasanrokijat and Rob Lammertink. Many thanks go out to Adrie Westphal and Willem van Berkel of the Biochemistry department at WUR, who have helped me with purifying laccase and with whom I had the pleasure to participate in interesting collaborative work. I would like to thank Peter Schoenmakers and Bert Wouters (University of Amsterdam) for their out-of-the-box discussions on laccase/microfluidics/material science that led to a partnership that, unfortunately, ended far too soon. Finally, the work as published in Chapter 5 would not have been possible without the prior knowledge generated from a strong collaboration with Elwin Vrouwe and the team of Micronit.

Many thanks go out to my opponents who kindly accepted the invitation to judge this thesis: Prof. Georg Gübitz, Prof. Erik Roesink, Dr Frank Hollmann and Prof. Willem van Berkel. I am looking forward to a lively discussion.

Mamma, ik had heel graag gewild dat je er bij kon zijn. Je bent zo'n geweldig mens, ik mis je heel erg en ik hou voor altijd van je.

Ik weet dat je het niet wil, maar je staat in het boekje... Bianca, ik hou van je! Ik denk dat we allebei in de laatste jaren heel erg gegroeid zijn. Je bent de slimste, liefste, leukste en mooiste persoon die ik ken, en ik zou serieus niet weten hoe ik het zou overleven zonder je. Je bent geweldig kleine Bran! 


\section{Publications}

Characterization of the laccase-mediated oligomerization of 4-hydroxybenzoic acid

S. Slagman, J. Escorihuela, H. Zuilhof, M. C. R. Franssen, RSC Adv. 2016, 6, 99367-99375

Laccase-Mediated Grafting on Biopolymers and Synthetic Polymers: A Critical Review

S. Slagman, H. Zuilhof, M. C. R. Franssen, ChemBioChem 2018, 19, 288-311

Elucidating the mechanism behind the laccase-mediated modification of poly(ethersulfone)

S. Slagman, W. A. J onkers, H. Zuilhof, M. C. R. Franssen, RSC Adv. 2018, 8, 27101-27110

One-Step Generation of Reactive Superhydrophobic Surfaces via $\mathrm{SiHCl}_{3}$-Based Silicone Nanofilaments

S. Slagman, S.P. Pujari, M.C.R. Franssen and H. Zuilhof 2018, accepted for publication in Langmuir 


\section{About the author}

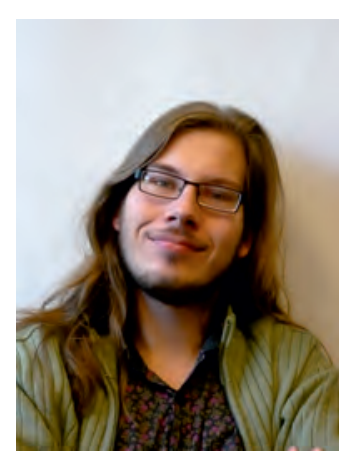

Sjoerd Slagman (August 21 ${ }^{\text {st }} 1990$, Hoorn - the Netherlands) started his academic career at the University of Amsterdam (UvA), where he enrolled in the Bachelor programme Chemistry in 2007. He finalised his bachelor thesis entitled "Synthesis of Recently Isolated Pyrrolizidine Alkaloids Using N-Acyliminium Ion Chemistry and Gold(I) mediated Cyclization" under supervision of Dr S. Kinderman at the laboratory of Prof. H. Hiemstra. He subsequently graduated from his Master's programme Chemistry (Molecular Design, Synthesis \& Catalysis) after a one-year internship in the group of Prof. J. van Maarseveen (UvA), during which he worked on a novel allylic amination/acyl-transfer cascade for the synthesis of cyclic peptides. In 2013 he started his PhD at the Laboratory of Organic Chemistry of Prof. H. Zuilhof (Wageningen University, the Netherlands). Under the guidance of Dr M. Franssen, he completed (2018) a challenging and highly interdisciplinary PhD trajectory regarding laccase-mediated surface modification, resulting in four first-author peer-reviewed scientific papers. He currently holds a postdoctoral position at the laboratory of Prof. Wolf-Dieter Fessner at TU Darmstadt (Germany) in which he studies novel enzymes for performing, among others, carboligation reactions. 


\section{Overview of completed training activities}

\section{Discipline specific activities}

\begin{tabular}{|c|c|c|c|}
\hline Course/ meeting & Organiser & Year(s) & Poster/ oral \\
\hline NWO Organic Chemistry Symposium & NWO & 2013 & \\
\hline CHAINS 2014 & NWO & 2014 & Poster \\
\hline CHAINS 2015 & NWO & 2015 & Poster + oral \\
\hline CHAINS 2016 & NWO & 2016 & Poster \\
\hline Wageningen SOC Symposium (Internat.) & KNCV, SOC & 2014 & \\
\hline Wageningen SOC Symposium (Nat.) & KNCV, SOC & 2015 & \\
\hline Wageningen SOC Symposium (Internat.) & KNCV, SOC & 2016 & \\
\hline Wageningen SOC Symposium (Nat.) & KNCV, SOC & 2017 & Oral \\
\hline Nanocity 2014 & NanoNextNL & 2014 & Poster \\
\hline Nanocity 2015 & NanoNextNL & 2015 & Poster \\
\hline Nanocity 2016 & NanoNextNL & 2016 & Poster \\
\hline New Frontiers in Synthetic Life & Radboud UMC & 2013 & \\
\hline Masterclass Applied Biocatalysis & VLAG & 2014 & \\
\hline Intro Membranes @ Pentair & Pentair X-Flow & 2016 & \\
\hline Oxizymes & VLAG & 2016 & Poster + oral \\
\hline Biotrans & MKE, SysBioCat & 2017 & Poster \\
\hline Lunch Lecture & Alchimica & 2016 & Oral \\
\hline Advanced Organic Chemistry & ORC & 2013-2017 & \\
\hline Advanced Chemistry & ORC, BNT, PCC & 2014-2016 & \\
\hline \multicolumn{4}{|c|}{ General courses } \\
\hline Course & Organiser & Year(s) & \\
\hline VLAG PhD Week & VLAG & 2014 & \\
\hline IP and Valorisation Awareness & NanoNextNL & 2015 & \\
\hline Time Management & WGS & 2017 & \\
\hline Career Perspectives & WGS & 2017 & \\
\hline Scientific Artwork with Photoshop and I Ilustrator & WGS & 2017 & \\
\hline \multicolumn{4}{|c|}{ Optional } \\
\hline Meeting & Organiser & Year(s) & \\
\hline Preparing PhD Research Proposal & - & 2013 & \\
\hline PhD Study Tour to Canada & ORC & 2015 & \\
\hline PhD Study Tour to Denmark and Sweden & ORC & 2017 & \\
\hline Group Meetings and Colloquia & ORC & 2013-2018 & \\
\hline NanoNext Project Meetings & NanoNextNL & 2014-2016 & \\
\hline
\end{tabular}


The research and training described in this thesis was financially supported by NanoNextNL project 04A. 01 "Biomimetic selective layers".

Financial support from Wageningen University for printing this thesis is gratefully acknowledged. 



\section{Propositions}

1. Polymers built up from non-conjugated electron-deficient phenylene units cannot be modified through laccase-mediated grafting.

(this thesis)

2. Growth of $\mathrm{SiHCl}_{3}$-based silicone nanofilaments provides a robust route towards functionalising predominantly inert PMMA.

(this thesis)

3. Machine-aided synthesis will not replace hands-on synthetic chemistry in the next 15 years.

4. Laccase does not catalyse any of the transformations suggested by Iqbal and co-workers. (Iqbal et al., Green Chem., 2015, 17, 3858-3869)

5. Racism cannot be justified by stating that group selection has, evolutionarily, been very important.

6. Preventive detention (in Dutch: TBS) should be included in every judicial sentence.

Propositions belonging to the thesis entitled "Mild Oxidation and Functionalisation of Synthetic Polymer Surfaces"

Sjoerd Slagman

Wageningen, 7 November 2018 